$\mathrm{UiO}$ : Institute of Theoretical Astrophysics University of Oslo

\title{
Quantized Vortices in Superfluid Dark Matter
}

Renate Mauland

Master's Thesis, Spring 2019

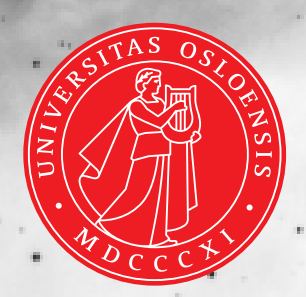


Copyright (c) 2019, Renate Mauland

This work, entitled "Quantized Vortices in Superfluid Dark Matter" is distributed under the terms of the Public Library of Science Open Access License, a copy of which can be found at https://journals.plos.org/plosone/s/licenses-and-copyright.

Cover Photo: Open Source Image from www.pexels.com. 


\begin{abstract}
In a recent paper by Berezhiani and Khoury [1], a superfluid dark matter (DM) model was presented. The model consists of light axion-like DM particles $(m \sim \mathrm{eV})$, which condense and form a superfluid at galactic scales. The superfluid exhibits collective excitations known as phonons, which interact with baryons and mediate a force similar to that of Modified Newtonian Dynamics (MOND). The superfluid DM model therefore combines the success of MOND at small scales with that of the $\Lambda$ CDM model at large scales. By analogy with superfluids studied in the lab, we expect a grid of vortices with quantized circulation to form in a rotating galaxy containing superfluid DM. In this thesis, we explore the properties of the superfluid DM vortices and their impact on the surroundings. We find that the vortex cores are small, $10^{-4}-10^{-3} \mathrm{~m}$, and that the vortices are separated by vast distances, $\sim 0.002 \mathrm{AU}$. The viable parameter space of the model is found to be substantial, and a reduction in the DM particle mass results in larger vortices with a higher energy. Yet, no combination of parameters explored here give vortices energetic enough to have a noticeable effect on the galaxy as a whole. However, we find that the baryons might affect the vortices instead, although further investigation is needed to understand the full extent of this result. As a first step towards a more complete model, we also extract a vortex equation from the relativistic Lagrangian describing the model. We find that a solution with the standard properties assumed for a vortex solution is unlikely to exist, at least for the parameter scales proposed in [1].
\end{abstract}




\section{Acknowledgments}

I would like to thank my supervisor, Øystein Elgarøy, for guiding me through the thesis work and answering all my silly questions. I would also like to thank the theoretical cosmologists of ITA for taking an interest in my thesis and contributing with valuable insight, help and wisdom. Thank you to the people of Stjernekjelleren, for all the awesome moments during my time there, and for keeping my social skills somewhat up to date. Of course, a great thanks to all my friends and family, who have supported me and helped me stay sane during the last five years. Lastly, a huge thank you to my fiancé for enduring my time as a student, and for keeping me well fed all these years. 


\section{Contents}

$\begin{array}{lll}\text { Abstract } & \text { iii }\end{array}$

Acknowledgments $\quad$ v

List of Figures viii

1 Introduction 1

2 A Brief History of Dark Matter 5

2.1 The $\Lambda$ CDM Model . . . . . . . . . . . . . . . . . 5

2.2 Dark Matter . . . . . . . . . . . . . . . 6

3 Condensate Theory 11

3.1 Bosons vs. Fermions . . . . . . . . . . . . . . . . . 11

3.2 The Bose-Einstein Condensate . . . . . . . . . . . . . . . . 12

3.2.1 The Critical Temperature . . . . . . . . . . . . . . . . 12

3.2.2 The Condensate Energy . . . . . . . . . . . . . . . . 16

3.3 Singularities in the Condensate . . . . . . . . . . . . . . 17

3.3 .1 Vortices . . . . . . . . . . . . . . . . . . 18

3.3 .2 The Energy of a Vortex . . . . . . . . . . . . . 18

3.4 Collective Excitations in the Condensate . . . . . . . . . . . . 23

3.5 BEC vs. Superfluid . . . . . . . . . . . . . . . . . . . 23

4 Superfluid Dark Matter $\quad \mathbf{2 7}$

4.1 Properties of Modified Newtonian Dynamics (MOND) . . . . . . . . . . 27

4.2 Two-Fluid Description . . . . . . . . . . . . . . . . . . 29

4.3 Quantum Field Theory Approach . . . . . . . . . . . . . . . . . . . . 32

4.3 .1 The Vortex Solution . . . . . . . . . . . . . . . 36

5 A Simple Model $\quad 39$

5.1 Gravitational Potential . . . . . . . . . . . . . . . . . . . . . 39

5.2 Vortex Properties . . . . . . . . . . . . . . . . . . . . . 41

5.3 Critical Temperature . . . . . . . . . . . . . . . . . 46 
6 Parameter Variation $\quad 49$

6.1 Condensate Halo Density Profile . . . . . . . . . . . . . . . . . . 50

6.1.1 Vortex Core Size ................... 53

6.2 One Meter Vortex Core .................... . 55

6.3 The $\alpha$ Parameter ........................ 64

6.4 Rotation Curves ...................... . . 65

6.5 DM Particle Mass Variation . . . . . . . . . . . . . . . . 70

7 Vortex-Baryon Interaction $\quad \mathbf{7 5}$

7.1 The Interaction Term . . . . . . . . . . . . . . . . . . . 75

7.2 The Equations of Motion . . . . . . . . . . . . . . . . 76

8 Superfluid Dark Matter Lagrangian $\quad 81$

8.1 The Klein-Gordon Equation . . . . . . . . . . . . . . . . . . . . . . 81

8.2 Equations of Motion . . . . . . . . . . . . . . . . . . 82

8.3 Interpretation ...................... . . 87

8.3.1 Background Information . . . . . . . . . . . . . 87

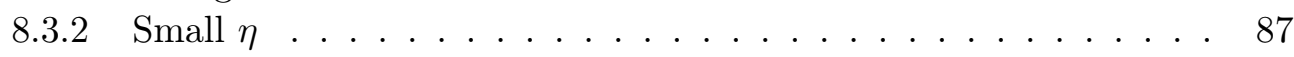

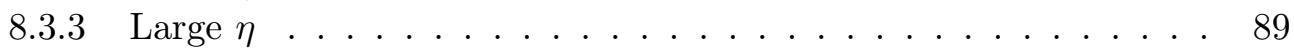

8.3.4 Numerical Stability . . . . . . . . . . . . . . . . 91

9 Discussion $\quad 97$

9.1 Chapter 5 - A Simple Model . . . . . . . . . . . . . . . . . 97

9.2 Chapter 6 - Parameter Variation . . . . . . . . . . . . . . 100

9.3 Chapter 7 - Vortex-Baryon Interaction . . . . . . . . . . . . . . . 105

9.4 Chapter 8 - Superfluid Dark Matter Lagrangian . . . . . . . . . . . . 106

10 Conclusions $\quad 109$

10.1 Future Work ...................... . . 111

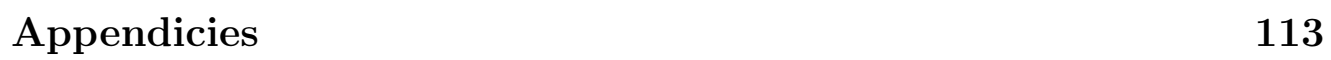

$\begin{array}{ll}\text { A Relativistic Lagrangian Calculations } & 115\end{array}$

B Numerical Methods $\quad 127$

B.1 The Shooting Method . . . . . . . . . . . . . . . . . . . 127

B.2 The solve_bvp Module . . . . . . . . . . . . . . . . . . 129

B.3 Newton's Method ........................ 130

$\begin{array}{ll}\text { Bibliography } & 133\end{array}$ 


\section{List of Figures}

4.1 Baryonic Tully-Fisher Relation. . . . . . . . . . . . . . . . . . . . . 30

4.2 Potential of Condensate - Spontaneous Symmetry Breaking. . . . . . . . 33

4.3 Profile of a Single Straight-Line Vortex. . . . . . . . . . . . . . . 37

5.1 Extent of Superfluid Component vs. Full Size of Dark Matter Halo. . . 44

5.2 Illustration of a Single Vortex at Galactic Center. . . . . . . . . . . . . 45

6.1 Zero-Temperature Condensate Dark Matter Density Profile. . . . . . . . 52

6.2 Coherence Length as a Function of Density. . . . . . . . . . . . . . 54

6.3 DM Density Profile Fitting for $1 \mathrm{~m}$ Coherence Length. . . . . . . . . . . 58

6.4 Cross Section Dependence of Thermalization Radius. . . . . . . . . . . 59

6.5 Cross Section Mass Constraint. . . . . . . . . . . . . . . . . 61

6.6 Toy Density Profiles for Rotation Curve Estimates. . . . . . . . . . . . . 66

6.7 Rotation Curves Produced by Toy Density Profiles. . . . . . . . . . . . . 69

6.8 Dark Matter Density Profile Fitting for Various Values of $m$. . . . . . . 72

8.1 Vortex Profile Close to Core. . . . . . . . . . . . . . . . . . . . 88

8.2 Vortex Profile Far from Core. . . . . . . . . . . . . . . . . . . . 90

8.3 Shooting Method Results. . . . . . . . . . . . . . . . . . . . . . 92

8.4 Shooting Method Results for Valid Values of $\Lambda_{c} \ldots \ldots \ldots 3$

8.5 Shooting Method Results - Zoom. . . . . . . . . . . . . . . . . 94

8.6 Example of "Good" Shot. . . . . . . . . . . . . . . . . . . . . . 95 



\section{Chapter 1}

\section{Introduction}

"The Universe is big. It's vast and complicated and ridiculous. And sometimes, very rarely, impossible things just happen and we call them miracles."

- The Doctor, Doctor Who: The Pandorica Opens.

Throughout the ages, humankind has wondered about the Universe. It has been a part of spiritual beliefs and religions, of myths and legends and of songs and poems. We have written exciting books, made astonishing movies and breathtaking art, all from our imagination and longing to unfold the mysteries of the cosmos. As time has gone by, we have found new ways of describing the Universe and our place in it. We have developed scientific methods and made thorough observations. We have constructed theories where the Universe is described through the language of mathematics, providing predictions that align impressively with what we can observe. Every day the scientific community keeps pushing our understanding of what goes on out there, all the way from discovering tiny new worlds to explaining the large-scale structure of the Universe. The task is challenging, and a lot is yet to be discovered.

One of the puzzles that remains unsolved is the nature of the matter content of the Universe. There is one kind of matter that can be observed and measured directly. It is the matter that makes up all our stars and planets, and it is the same matter that makes up us. This is the baryonic matter, or luminous matter, and we seem to understand it quite well. However, based on observations and our (currently) most trusted theory, there must exist yet another kind of matter. One we cannot see, a dark matter. It is the nature of this dark matter that is unknown to us.

One theory that incorporates dark matter as well as baryonic matter is the $\Lambda$ CDM model. This model is today widely accepted as the standard model of Big Bang cosmology. It includes cold dark matter (CDM) and a cosmological constant, $\Lambda$, describing dark energy - the key to understanding the accelerated expansion of the Universe. The model first appeared during the 1990s and gained momentum as the accelerating Universe idea was supported by observations [2,3]. Experiments focusing on the Cosmic Microwave Background (CMB) also delivered results consistent with its predictions $[4,5]$. The $\Lambda \mathrm{CDM}$ model assumes general relativity, developed by Albert Einstein 
between 1907-1915, to be the correct gravitational theory on cosmic scales, and it naturally explains the structures in the CMB, the large scale structure of the cosmos, the abundances of hydrogen, helium and lithium, and also, as stated above, the accelerated expansion of the Universe. In addition, the $\Lambda$ CDM model does not depend upon the nature of dark matter, only its presence, and most dark matter discoveries would therefore go quite well with the theory.

So far, all seems well. The $\Lambda$ CDM model describes the large cosmos quite exceedingly, and if we could understand the dark matter (and energy) we would have a solid description of not only how our Universe has evolved, but also what it consists of. There is, however, a problem. The $\Lambda \mathrm{CDM}$ model has difficulties reproducing observations at smaller scales. For this reason, some astrophysicists believe that the model needs to be changed, or alternatively, that the theory of gravity needs to be modified. Already before the $\Lambda \mathrm{CDM}$ model gained momentum, a paper was published where the regular Newtonian laws were adjusted on galactic scales [6], leaving dark matter redundant. As it so happens, this slight adjustment is what we need to reproduce the small scale observations that the $\Lambda \mathrm{CDM}$ model fails to explain, such as the Baryonic Tully-Fisher Relation or the satellite phase-space correlation challenge [7, 8]. This other model goes by the name of MOdified Newtonian Dynamics, or MOND for short.

Again, there is a problem. MOND is in itself not a full theory, only an effective equation. Attempts at constructing full theories with MOND as a limit has failed to sufficiently explain the Universe at large scales. As it stands now, it is almost as if the MOND and $\Lambda$ CDM models each predicts what the other cannot. The ideal situation would be a model where $\Lambda$ CDM was used to describe the Universe at large scales, and MOND at small. There have been made several attempts at this before [8, 9], but none have turned out to be completely satisfying. Most of these models also describe MOND and CDM as separate components, when ideally we would like them to be two effects naturally arising from the same source. An additional problem is that MOND is slightly $a d-h o c$ - there is not presented any reason for why Newton's gravitational law should change at galactic scale ${ }^{1}$. This would, of course, have to be thoroughly explained in a fully functional theory incorporating MOND.

There still might be another way to go. What if we could keep the $\Lambda$ CDM model, but still obtain MOND-like results on galactic scales? In a paper published in 2016 [1], written by Lasha Berezhiani and Justin Khoury, a model describing superfluid dark matter was proposed. The idea is that dark matter is a bosonic self-interacting, axionlike particle, which thermalizes and condenses into a superfluid at galactic scales. In the superfluid state, the dark matter is macroscopically explained as collective modes, also known as phonons (section 3.4). These phonons will mediate a MOND-like force when interacting with baryons, and thus reproduce MONDian results on galactic scales. Still, there is the need for preserving the $\Lambda \mathrm{CDM}$ behavior at larger scales. This is accomplished through the two-fluid description of the superfluid phenomenon (see sec-

\footnotetext{
${ }^{1}$ MOND only changes Newton's second law (N2) for accelerations lower than a certain constant, $a_{0}$, when the force in question is gravity. For other forces, N2 has been tested for accelerations way below $a_{0}$, and no deviations have been found [10].
} 
tion 4.2), where the fluid is divided into a normal and a superfluid component. In the model presented by Berezhiani and Khoury, structures on larger scales, such as galactic clusters, will be almost or entirely in the normal state. In this state, the dark matter behaves as regular CDM particles, preserving the $\Lambda$ CDM predictions. In other words, this model has CDM and MOND naturally appearing from the same source, without any need for a modification of gravity.

The model proposed by Berezhiani and Khoury results in a number of predictions, in addition to reproducing existing observations $[1,11]$. Among these is the existence of vortices in the superfluid dark matter. To understand the behavior of the superfluid, the current knowledge within the study of cold atom physics and Bose-Einstein condensates is quite useful. In experiments done in this particular branch of physics, the studied condensates are held in place by magnetic traps. In our case, however, it is gravity that encapsulates the superfluid. It has been shown (see section 3.3) that rotating traps result in vortices forming in the condensates. As it so happens, a galaxy rotates, and assuming that the dark matter halo rotates with it, one should expect to see vortices form in the dark matter superfluid. A few calculations and remarks were included in [1] concerning this topic, but much is still left unexplored. The goal of this thesis us thus to investigate the properties and possible impact of vortices on the dynamics of spiral galaxies. This will first be done by using a simplified model, which has the bonus of allowing us to analyze the parameter space left largely unexplored by the authors of [1]. We will then take steps to extend the investigation to the full, relativistic Lagrangian of the model.

We start off this thesis by a short historical introduction to dark matter (chapter 2) and proceed by presenting all necessary background theory from the field of condensed matter physics (chapter 3). This is followed by an introduction to the two-fluid and field theory description of a superfluid in chapter 4, together with a motivation for why we want a superfluid dark matter model. In chapter 5 we present a simplified dark matter halo and calculate some key properties related to the vortices that form in the rotating superfluid. In chapter 6 we further look at the main parameters of the model presented in [1], and see how changing them impacts the model as a whole, the vortices and their impact on the surroundings. We then move on to study the interaction between vortices and baryons in chapter 7, focusing on how the baryons affect the vortices. Finally, in chapter 8, we search for a vortex solution of the full relativistic Lagrangian of the model, presented in section 6 of [1]. We finish off by discussing our results in chapter 9, and lastly we present our conclusions in chapter 10 . 


\section{Chapter 2}

\section{A Brief History of Dark Matter}

"We're all stories, in the end. Just make it a good one, eh?"

- The Doctor, Doctor Who: The Big Bang.

The introductory chapter of this thesis states that the $\Lambda$ CDM model is the standard model of Big Bang cosmology. After this statement, we move on to comparing it to MOND, which gets its own explanation in section 4.1. For an astrophysicist, the $\Lambda$ CDM model is well known, and any further description might be unnecessary. However, the reader of this thesis might find themselves wondering about this $\Lambda$ CDM model after all, and more importantly, might find themselves wondering about the components within it. This thesis concerns the behavior and nature of dark matter, and one might wonder at which point the whole scientific community decided that an invisible, non-baryonic and completely unknown matter must be the answer to all our troubles. This chapter aims to address some of these questions, especially focusing on the history of dark matter itself.

\subsection{The $\Lambda$ CDM Model}

The $\Lambda$ CDM model is the standard model of Big Bang cosmology. This essentially means that it is the best current theory explaining the past, present and future behavior of the Universe as we know it. It supports the idea that the Universe started in The Big Bang, which before there was "nothing," and that we live in a Universe with accelerated expansion. The driving mechanism behind this accelerated expansion, whatever it may be, has been given the name Dark Energy. The cosmological constant, $\Lambda$, is believed to be the simplest way to explain dark energy, and it appears in Einstein's field equations as the energy density of space. The $\Lambda \mathrm{CDM}$ model also explains the structure of the cosmos, and thus the formation of galaxies and the clusters they inhabit. A large part of this explanation lies in the CDM part of $\Lambda \mathrm{CDM}$, that is, Cold Dark Matter. In other words, the theory describes a Universe consisting of dark energy $(\Lambda)$ and dark matter

\footnotetext{
${ }^{1}$ Simply meaning that our theories do not allow us to calculate beyond this point.
} 
$(\mathrm{CDM})$, in addition to regular baryonic matter. In this thesis, dark matter is essential, and we will therefore explain its history further in the section below. This is mainly all that is needed to know regarding the $\Lambda \mathrm{CDM}$ model for our purposes ${ }^{2}$, with the exception of observations it fails to reproduce. This will be revisited in section 4.1.

\subsection{Dark Matter}

This section presents a short version of the history of dark matter, closely following the works of Bertone and Hooper in [13]. For further details, please consult the aforementioned paper.

The idea of something strange and invisible is not new to us. For a long time, the humankind has looked upon the starry sky with the naked eye, wondering what could be up there. All around the globe, people have seen the stars and the planets, separating them apart based on their behavior and not their looks. When Galileo had the idea of viewing the sky through his telescope in the early 1600s, he was presumably the first to see countless new stars, in addition to his discovery of the four largest moons of Jupiter. This showed us something important - the world around us contains objects that we may not be able to see. At the same time, it also told us that what is invisible to us now, might only be so until we acquire the correct tools to find it.

When Newton in 1687 published his Philosophice Naturalis Principia Mathematica [14], he showed that it was possible to find the gravitational mass of an object based on its movements. Almost 100 years later, John Michell argued that if light was affected by gravity, the existence of objects so heavy that light would be unable to escape should be possible [15]. Now we know this as a black hole, but at the time this was merely an invisible object that might be out there somewhere. It is also well known that Neptune was discovered based on the strange behavior of Uranus, and that a new planet, "Vulcan," was proposed to explain the perihelion precession of Mercury. In other words, invisible objects have been proposed before, and they have in some cases been used to explain the behavior of observed astronomical bodies that deviate from what we expect. In the case of Mercury's perihelion, however, it took Einstein's theory of general relativity to explain it, and no extra planet was ever discovered.

There have been mentions of dark planets, dark stars and dark clouds in scientific papers for a long time. Dark clouds, or nebulae, became a discussion in the later half of the 1800s, especially after astronomical photography was invented. Lord Kelvin was one of the first people who tried to estimate the amount of dark matter in the Milky Way [16, 17], and Henri Poincaré argued in 1906, based on Kelvins predictions, that the amount of dark matter should be less than the amount of luminous matter [18]. At this point, we must remember that dark matter is not the same to them as it is to us. When using the words "dark matter," they imagined dark stars and gases which was invisible to the naked eye, not a new type of non-baryonic particle as we do today.

In the early 1900s, quite a few scientists studied the relationship between the motion of stars in our galaxy and their velocity dispersion, together with an estimate of the

\footnotetext{
${ }^{2}$ More information may be found in textbooks such as [12].
} 
effective stellar mass. In the end, this gave an estimate of the density of matter close to the Sun, where they also addressed the density of dark matter. This work woke an interest in determining the density of dark matter, especially on a local scale.

At this point in history, we arrive at the man who probably is the most mentioned in relation to the rise of the dark matter field: Fritz Zwicky. From his study of the velocity dispersion of galaxies within several galaxy clusters in $1933[19,20]$, he discovered that the velocity dispersion within the Coma Cluster was larger than what was expected, at least when he used the predicted mass of a galaxy rooted in the amount of luminous matter. Based on his findings, it had to be the case that dark matter existed in greater measures than luminous matter. This was new because, as mentioned above, one thought at this point that luminous matter dominated the galaxies. Of course, the dark matter was still assumed to be cold stars, some form of solid bodies and different gases, as mentioned in Zwicky's follow up paper from 1937 [21]. In 1936, Sinclair Smith also published a paper concerning the mass of galaxy clusters [22], and estimated the mass of a single galaxy to be around $10^{11} M_{\odot}$, in contrast to previous estimations by Hubble that gave $10^{9} M_{\odot}[23]$. Hubble later cited the works of Smith and acknowledged that the difference in mass estimated by his study of rotation of galaxies, and Smiths use of dynamics, indeed was quite "real and important" [24].

The findings of both Zwicky and Smith were challenged by the community in the years that followed. The only explanation of their results was that a dominant amount of the mass in galaxy clusters was due to dark matter, that could not be observed. This was of course hard to swallow and seemed unlikely. One of the more important quantities to calculate at this time thus became the mass-to-light ratios of galaxy clusters, and when the late 1950s arrived, a lot of papers on this topic in particular had been published. The results of these papers were mainly very large mass-to-light ratios, which built up under the suspicion that the clusters were packed with invisible matter. The hypothesis of dark matter was not accepted in the community, nor was it dismissed, but astronomers tried to find other explanations. This could be ideas like instabilities in the clusters, or that some galaxies were not a part of the system, and should not be taken into account when calculating the velocity dispersions. At the very least, the community was in agreement that further information would be necessary to understand the differences in mass that had been discovered. Still, people gradually became more willing to discuss what this dark matter might be made of, which led to gas being ruled out in the late 60s/early 70s. Astronomers then continued by looking for other possibilities, like collapsed objects or dwarf stars.

One of the observations that has been particularly important in the dark matter journey is the galactic rotation curves. Already in 1917, the rotation curve of Andromeda was measured [25], showing an approximately constant angular velocity. However, the rotation curve was only measured from the center and out to 2.5 arcminutes, while the visible part of Andromeda measures $178 \times 63$ arcminutes in the sky [26]. In his 1937 paper [21], Fritz Zwicky looked at using the rotation curves to determine the mass of galaxies, but concluded that further information was necessary. In 1939, Horace Babcock measured the rotation curve of Andromeda out to 100 arcminutes from its center, and used it to calculate the mass distribution of the galaxy [27]. He found 
very large values of the circular velocity at high radii, which he recognized as a sign of huge amounts of unseen mass at the edge of the galaxy. However, he did not argue for the existence of dark matter, but rather the need for new information regarding the dynamics, which would lead to a smaller amount of mass in the outskirts.

After the Second World War, a lot of radio technology was redirected to scientific use, and in 1957 the first radio rotation curve of M31 was published [28]. The observations extended all the way out to two degrees from the center (120 arcminutes). In 1959, the combined mass of the Milky Way and Andromeda was calculated by Kahn and Woltjer [29], by proposing that the two galaxies were part of a bound system. The result was a combined mass six times larger than the accepted value at this time. When looking at this result from today's perspective, it is in fact quite a clear sign of dark matter halos around galaxies. Despite the findings of these flat rotation curves, which indicated a larger amount of matter in the outskirts of Andromeda than what was observed, astronomers did not seem to think that it conflicted with the understanding of galaxies in the late 1950s. This, however, changed around a decade later.

We now reach yet another well-known name within the dark matter field: Vera Rubin. In the 1960s, Kent Ford and Vera Rubin observed the Andromeda galaxy with an image tube spectrograph made by Ford. Together they published a paper in 1970, containing a flattening M31 rotation curve of greater quality than ever before [30]. In the same year, Ken Freedman concluded that also for M33 and NGC300 (two galaxies in our local group) the rotation curves reached a maximum further out that what was predicted by the amount of luminous matter [31]. He further stated that if his data was correct, the existence of additional undetected matter must be the case, and it had to have a different distribution in the galaxy than the optical matter. This is perhaps the first time that the existence of additional matter is actually argued for, instead of just mentioned as an unlikely possible explanation of the observations. In 1972, Rogstad and Shostak analyzed the rotation curves of five other galaxies and saw that the rotation curves flattened out, and stayed flat, up until the largest observed radii [32]. They reached the same conclusions as Freedman, and confirmed the need for invisible matter in the outer regions of the galaxies.

One of the first scientists to reach a modern interpretation of the flattening rotation curves was Morton Roberts. In 1972/73, he argued that the flattening of the rotation curves indicated a large mass density in the outer regions, and that we could not expect the last measured point to actually define the edge of a galaxy [33, 34]. It had to be assumed that a galaxy was larger than what was found by regular means, and also that the mass-to-light ratio increased with radius. Still, he later said that the missing mass could be dwarf stars [35], showing that even though he was one of the first to understand the distribution of dark matter in galaxies, he still did not expect the excess matter to be anything but baryonic.

Throughout the 70s, people continued publishing papers where the rotation curves of new galaxies were studied, all showing a flattening with large radii. The evidence of a huge amount of unseen matter was building up, and the community could no longer ignore the fact that it seemed like galaxies contained massive halos of some sort, reaching way beyond the luminous matter. In 1978, the Ph.D. thesis of Albert Bosma 
was published [36]. This showed the rotation curves of 25 galaxies, where the vast majority had flat rotation curves extending beyond the luminous galactic disk. This again strengthened the evidence towards more mass in the outskirts of the galaxies. Not long after Rubin, Ford and Thonnard published a paper showing the rotation curves of ten high-luminous spiral galaxies [37]. These were also flat all the way to the furthest measured radius, and the paper went on to become one of the most well-known and cited in the field. In 1979, a paper reviewing the galaxy masses and their mass-to-light ratios was published by Faber and Gallagher [38]. Here it was stated that the existence of large amounts of invisible matter in the Universe was becoming more and more likely.

This is essentially the story of how something like huge amounts of invisible matter in the Universe became a fact. Since the review paper in 1979, a large amount of studies have been performed within the dark matter field, and the theory explaining how galaxies and clusters are held together has changed accordingly. Today we no longer expect invisible gas or dwarf stars to make up the amount of missing matter, as observations have ruled these options out. Now we talk about non-baryonic particles, something entirely different from the elementary particles of the standard model. We know that the dark matter is there, as we need it to understand and make sense of our observations, but we are still to figure out exactly what it is. We do know that the particles must be non-interacting, or weakly interacting, with themselves, and that they only interact with ordinary (baryonic) matter through gravity. In the last 30 or so years, many dark matter candidates have been proposed, ranging from WIMPs (Weakly Interacting Massive Particles) to MACHOs (MAssive Compact Halo Objects) and primordial black holes. Another dark matter candidate has been axions, which are hypothetical low mass elementary particles that initially were introduced to solve a problem experienced in QCD (Quantum ChromoDynamics) ${ }^{3}$. In the model proposed by Berezhiani and Khoury, the dark matter is expected to be axion-like in order to have superfluid properties.

Now that the dark matter part of the new superfluid dark matter model has been placed in a historical context, it is time to delve into the world of Bose-Einstein condensates, in order to further understand the superfluid part of the model presented in [1].

\footnotetext{
${ }^{3}$ For an overview of dark matter candidates see section V and VI in [13].
} 


\title{
Chapter 3
}

\section{Condensate Theory}

\author{
"Just had a fall. All the way down there, right to the library. Heck of a climb \\ back up."
}

- The Doctor, Doctor Who: The Eleventh Hour.

This chapter gives a short description of the Bose-Einstein condensate theory needed to understand the behavior and characteristics of a superfluid. The phenomenon of superfluidity is in itself closely coupled to the Bose-Einstein condensate (BEC), which is why it is highly relevant to our study. Indeed, not all cases of BECs need have the superfluid property. The BEC formed in a non-interacting gas, for example, does not exhibit the superfluid property, as it turns out that interactions are a vital part of the superfluid construct. For our applications, the superfluidity is needed, and it will thus be assumed in our description of the BEC. The presentation given here follows that of Pethick and Smith [39].

Section 3.1 briefly explains what distinguishes bosons from fermions, and section 3.2 moves on to introduce the concept of a Bose-Einstein condensate, the critical temperature and the condensate energy. Section 3.3 concerns singularities that may form in a rotating superfluid, describing their form and properties in detail. Section 3.4 briefly outlines one type of excitation that could arise in a condensate, namely the phonon. Finally, section 3.5 touches upon the topic of what makes a BEC into a superfluid.

\subsection{Bosons vs. Fermions}

All particles carry with them certain properties such as mass and electric charge. Another one of these properties is the particle spin, an intrinsic form of angular momentum. Particles are divided into two different groups: those who have half-integer spin and those who have integer spin. The half-integer spin particles are known as fermions, and the quarks and leptons of the standard model belong to this group. The integer spin particles are known as bosons, and the force carriers (such as the photon and the Higgs boson) make up this group. 
The bosons and fermions have very different properties. While the bosons follow Bose-Einstein statistics, the fermions follow Fermi-Dirac statistics. From this, the average number of bosons/fermions in a single particle state (in thermodynamical equilibrium), $\nu$, is given as:

$$
f_{\text {boson }}=\frac{1}{e^{\left(\epsilon_{\nu}-\mu\right) / k T}-1}, \quad f_{\text {fermion }}=\frac{1}{e^{\left(\epsilon_{\nu}-\mu\right) / k T}+1} .
$$

Here $\epsilon_{\nu}$ is the energy of the state $\nu$, and $\mu$ is the chemical potential, describing the energy change of the system in response to the addition or subtraction of a single particle.

The fermions obey the Pauli exclusion principle, which states that two or more identical fermions may not occupy the same quantum state [40]. This is the same as requiring the wavefunction describing a system of fermions to be antisymmetric under interchange of any two particles. In simple form, let us write it as $f(x, y)=-f(y, x)$, where $x$ and $y$ are particle states. This means that the function must be zero for $x=y$, which is the Pauli exclusion principle: two identical fermions may not populate the same state. This is not the case for bosons, where any number of identical bosons may occupy the same state, and the wavefunction is symmetric under particle interchange. This is also why we need bosons to create a condensate, as the condensate consists of particles in the same energy state ${ }^{1}$. This will be further explained in the following section concerning Bose-Einstein condensates.

\subsection{The Bose-Einstein Condensate}

A Bose-Einstein condensate is a state that occurs when a bosonic gas or fluid is cooled down to a temperature close to the point of absolute zero. At this point, the atoms in the gas will start to cluster in phase space, and (almost) all of them will enter the same, lowest energy state. The temperature needed to achieve this state is called the critical temperature, and it is related to the number density of the gas and the mass of the particles it consists of. The behavior of the condensate can be quite complicated and depends on the particles and their interactions. In addition, the gas might be subject to a spatial confinement as the result of a trapping potential. In experiments, this potential may often be described by a harmonic oscillator potential,

$$
V(x, y, z)=\frac{1}{2} m\left(\omega_{x}^{2} x^{2}+\omega_{y}^{2} y^{2}+\omega_{z}^{2} z^{2}\right) .
$$

\subsubsection{The Critical Temperature}

A Bose-Einstein condensate is a macroscopic occupation of the lowest possible energy state in a cloud of bosonic gas, and it occurs when the atoms are cooled down to below some critical temperature. This critical temperature can, as a first approach, be

\footnotetext{
${ }^{1}$ However, fermions may create pairs, and in that way behave as bosons, enabling them to form a condensate [41].
} 
estimated through dimensional analysis. A uniform gas of free, non-interacting particles may be described through the particle mass, $m$, the number density, $n$, and the Planck constant, $h$. The unique combination

$$
\frac{\hbar^{2} n^{2 / 3}}{m}
$$

has the dimension of energy. An estimate of the critical temperature can thus be given as

$$
T_{c}=C \frac{\hbar^{2} n^{2 / 3}}{m k},
$$

where $C$ is a dimensionless constant and $k$ is the Boltzmann constant. However, to find $C$, some calculation is required. This is what will be done in this section.

The amount of atoms occupying an energy state is related to the temperature of the gas. For non-interacting bosons in thermodynamical equilibrium, the average occupation number of a single particle state, $\nu$, is described by the Bose-Einstein distribution function:

$$
f^{0}\left(\epsilon_{\nu}\right)=\frac{1}{e^{\left(\epsilon_{\nu}-\mu\right) / k T}-1},
$$

as presented in the previous section. In addition to equation (3.5), we need an expression for the density of states. The expression used in the following can not be used to describe the condensate itself, but it may describe excited states, which is indeed what we need to obtain the desired results. The density of states is in general given by

$$
g(\epsilon)=\frac{\mathrm{dG}(\epsilon)}{\mathrm{d} \epsilon},
$$

and it tells us how many states are available to be occupied within an energy interval of $\mathrm{d} \epsilon$, at each energy level. Here, $G(\epsilon)$ is the total number of states with an energy less than $\epsilon$. For a gas in a box, the density of states is given as

$$
g(\epsilon)=\frac{V m^{3 / 2}}{2^{1 / 2} \pi^{2} \hbar^{3}} \epsilon^{1 / 2},
$$

where $V$ is the volume of the system. As mentioned earlier, the gases used in experiments are often confined by harmonic potentials, given by equation (3.2). In this case, the density of state is given by

$$
g(\epsilon)=\frac{\epsilon^{2}}{2 \hbar^{3} \omega_{x} \omega_{y} \omega_{z}},
$$

where the $\omega$ 's represent the oscillator frequency in different directions. It is common to write the density of states on the form

$$
g(\epsilon)=C_{\alpha} \epsilon^{\alpha-1},
$$


where $\alpha$ is some constant characteristic for the given trapping potential. For a gas in a box $\alpha=3 / 2$ and for a gas trapped by a harmonic oscillator potential $\alpha=3$. The values of the constants $C_{3 / 2}$ and $C_{3}$ can then be extracted from equation (3.7) and (3.8).

We now have the necessary background to calculate the critical temperature. If we look at a large system with a high number of particles, $N$, the effect of adding a particle is negligible, and we can put $\mu=0$. The effect of this is the same as neglecting the energy of the ground state. This will have some consequences, and we will revisit this at the end of this section. As for now, we use the approximation $\mu=0$. The number of particles in excited states (excited compared to the condensate state) is given by

$$
N_{\text {ex }}=\int_{0}^{\infty} d \epsilon g(\epsilon) f^{0}(\epsilon) .
$$

The integral goes over all possible energies, including the lowest energy state. However, as we have put $\mu=0$, the lowest energy state will not contribute and equation (3.10) still only describes the excited states. The critical temperature informs about the transition where all the atoms go from excited states to a large fraction being in the lowest energy state. The critical temperature may thus be calculated by putting all the particles in excited states:

$$
N=N_{\mathrm{ex}}\left(T_{c}, \mu=0\right)=\int_{0}^{\infty} d \epsilon g(\epsilon) \frac{1}{e^{\epsilon / k T_{c}}-1} .
$$

Using equation (3.9) and $x=\epsilon / k T_{c}$, equation (3.11) may be written as

$$
N=C_{\alpha}\left(k T_{c}\right)^{\alpha} \int_{0}^{\infty} d x \frac{x^{\alpha-1}}{e^{x}-1} .
$$

Expanding the distribution function in powers of $e^{-x}$, and using the known relation

$$
\int_{0}^{\infty} d x x^{\alpha-1} e^{-x}=\Gamma(\alpha),
$$

results in

$$
\int_{0}^{\infty} d x \frac{x^{\alpha-1}}{e^{x}-1}=\Gamma(\alpha) \zeta(\alpha) .
$$

Here $\Gamma$ is the ordinary gamma function, and $\zeta$ is the Riemann-zeta function,

$$
\zeta(\alpha)=\sum_{n=1}^{\infty} n^{-\alpha}
$$

Combining equation (3.12) and (3.14) we then get

$$
N=C_{\alpha} \Gamma(\alpha) \zeta(\alpha)\left(k T_{c}\right)^{\alpha},
$$


which gives the critical temperature

$$
k T_{c}=\frac{N^{1 / \alpha}}{\left[C_{\alpha} \Gamma(\alpha) \zeta(\alpha)\right]^{1 / \alpha}} .
$$

For a gas in a box, $\alpha=3 / 2$, equation (3.17) gives

$$
k T_{c} \approx 3.31 \frac{\hbar^{2} n^{2 / 3}}{m},
$$

which is on the form predicted in equation (3.4). For a gas trapped in a harmonic potential, $\alpha=3$, the critical temperature is given as

$$
T_{c} \approx 4.5\left(\frac{\bar{f}}{100 \mathrm{~Hz}}\right) N^{1 / 3} \mathrm{nK},
$$

where

$$
\bar{f}=\bar{\omega} / 2 \pi, \quad \bar{\omega}=\left(\omega_{x} \omega_{y} \omega_{z}\right)^{1 / 3} .
$$

We earlier put $\mu=0$ and decided to deal with the consequences of this later. Well, now is later. If our system had infinitely many particles, this approximation would be adequate, but as this is not the case, ignoring the ground state energy will affect the critical temperature. In reality $\mu=\epsilon_{\min }$, and for a harmonic oscillator potential this amounts to

$$
\epsilon_{\min }=\frac{3 \hbar \omega_{m}}{2},
$$

where $\omega_{m}=\left(\omega_{x}+\omega_{y}+\omega_{z}\right) / 3$. Based on our previous assumptions, we now have a change in the minimum energy corresponding to

$$
\Delta \epsilon_{\min }=\frac{3 \hbar \omega_{m}}{2}-0
$$

and thus a change in the chemical potential

$$
\Delta \mu=\Delta \epsilon_{\min } .
$$

This results in a change in the critical temperature given by

$$
\Delta T_{c}=-\frac{\zeta(2)}{3 \zeta(3)} \frac{\Delta \mu}{k}=-\frac{\zeta(2)}{2 \zeta(3)} \frac{\hbar \omega_{m}}{k} .
$$

Equation (3.24) originates from a specific heat capacity calculation in the vicinity of $T_{c}$, performed in [39] (their chapter 2), and we refer you to this work for further details. For the harmonic trap, this then results in a relative change of the critical temperature given as

$$
\frac{\Delta T_{c}}{T_{c}} \approx-0.73 \frac{\omega_{m}}{\bar{\omega}} N^{-1 / 3} .
$$

In other words, the critical temperature slightly decreases when the lowest energy state is included. This effect increases as the number of atoms in the system decreases. Still, this might also be strongly regulated by the choice of trap frequency, which is evident from equation (3.25). In addition, including interactions in the above consideration will tend to lower the critical temperature by a few percent [42]. 


\subsubsection{The Condensate Energy}

To describe the complex state that is Bose-Einstein condensation, the condensate wave function is used. As a BEC consists of atoms macroscopically occupying the same energy state, the whole system may be described through one single wave function, whose form is introduced in equation (3.28). To find the energy of this state, the Hamiltonian is needed, and in general it looks like this:

$$
H=\sum_{i=i}^{N}\left[\frac{\boldsymbol{p}_{i}^{2}}{2 m}+V\left(\boldsymbol{r}_{i}\right)\right]+U_{0} \sum_{i<j} \delta\left(\boldsymbol{r}_{i}-\boldsymbol{r}_{j}\right) .
$$

Each particle is denoted by $i$, so that $\boldsymbol{p}_{i}$ and $\boldsymbol{r}_{i}$ are the momentum and position of each particle, respectively. $V$ is the trapping potential, and $U_{0}$ is the two-body interaction energy of the particles. The latter is assumed to be a contact interaction. The energy of the system is then the expectation value of the Hamilton operator and is given as

$$
E(\psi)=\int d \boldsymbol{r}\left[\frac{\hbar^{2}}{2 m}|\nabla \psi(\boldsymbol{r})|^{2}+V(\boldsymbol{r})|\psi(\boldsymbol{r})|^{2}+\frac{1}{2} U_{0}|\psi(\boldsymbol{r})|^{4}\right],
$$

where $\psi$ is the condensate wave function,

$$
\psi=f e^{i \phi},
$$

written as a function of the modulus, $f$, and phase, $\phi$. The most favorable form of the wave function is then found by minimizing the energy under the condition that the number of particles be held constant. This is the same as minimizing the chemical potential times the number of particles subtracted from the energy $(E-\mu N)$, and the result is the now well established time independent Gross-Pitaevskii equation,

$$
-\frac{\hbar^{2}}{2 m} \nabla^{2} \psi(\boldsymbol{r})+V(\boldsymbol{r}) \psi(\boldsymbol{r})+U_{0}|\psi(\boldsymbol{r})|^{2} \psi(\boldsymbol{r})=\mu \psi(\boldsymbol{r}) .
$$

Here $\mu$ is the chemical potential. This equation describes how the condensate wave function changes as a function of space. The corresponding time dependent GrossPitaevskii equation is given as

$$
i \hbar \frac{\partial \psi(\boldsymbol{r}, t)}{\partial t}=-\frac{\hbar^{2}}{2 m} \nabla^{2} \psi(\boldsymbol{r}, t)+V(\boldsymbol{r}, t) \psi(\boldsymbol{r}, t)+U_{0}|\psi(\boldsymbol{r}, t)|^{2} \psi(\boldsymbol{r}, t) .
$$

See chapters 6 and 7 in [39] for more details on this in particular.

To understand the movement of the condensate, the velocity field is of great importance. This can be further evaluated through the continuity equation, which is obtained by multiplying the time dependent Gross-Pitaevskii equation by the complex conjugated wave function, and subtracting the complex conjugate of the resulting equation. This leads to

$$
\frac{\partial|\psi|^{2}}{\partial t}+\nabla \cdot\left[\frac{\hbar}{2 m i}\left(\psi^{*} \nabla \psi-\psi \nabla \psi^{*}\right)\right]=0
$$


From the wave function, the number density of the condensate can be defined as

$$
n=|\psi|^{2},
$$

and equation (3.31) is thus the number density continuity equation,

$$
\frac{\partial n}{\partial t}+\boldsymbol{\nabla} \cdot(n \boldsymbol{v})=0
$$

if the condensate velocity is defined as

$$
\boldsymbol{v}=\frac{\hbar}{2 m i} \frac{\left(\psi^{*} \boldsymbol{\nabla} \psi-\psi \boldsymbol{\nabla} \psi^{*}\right)}{|\psi|^{2}} .
$$

If the condensate wave function is then written as a function of its amplitude, $f$, and phase, $\phi$, as in equation (3.28), we get

$$
n=f^{2}
$$

and

$$
\boldsymbol{v}=\frac{\hbar}{m} \boldsymbol{\nabla} \phi,
$$

showing that the condensate velocity is proportional to the gradient of the phase, $\phi$.

\subsection{Singularities in the Condensate}

As a result of the form of equation (3.36), demonstrated in section 3.2.2, the velocity field of the condensate is irrotational

$$
\boldsymbol{\nabla} \times \boldsymbol{v}=0,
$$

except in the case of a singularity. The change in the phase of the wave function may be written as

$$
\Delta \phi=\oint \nabla \phi \cdot d \boldsymbol{l},
$$

which is known as the gradient theorem, or the fundamental theorem of calculus for line integrals [43]. This further means that the change in the phase of the wave function around a closed curve must be a multiple of $2 \pi$ :

$$
\Delta \phi=\oint \nabla \phi \cdot d \boldsymbol{l}=2 \pi \ell,
$$

where $\ell$ is a non-negative integer and $l$ parametrizes the curve. If the curve encloses a singularity, the integral will have a non-zero value. Otherwise, it will be zero. This is a result of Cauchy's integral theorem (see section 14.3 of [44]). 
The circulation around a closed curve is defined as

$$
\Gamma=\oint \boldsymbol{v} \cdot d \boldsymbol{l},
$$

which for our velocity field results in

$$
\Gamma=\oint \boldsymbol{v} \cdot d \boldsymbol{l}=\oint \frac{\hbar}{m} \boldsymbol{\nabla} \phi \cdot d \boldsymbol{l}=\frac{\hbar}{m} 2 \pi \ell .
$$

In other words, the circulation is quantized.

\subsubsection{Vortices}

Now, the question that needs answering is aimed towards the singularities: what are they? To get the information we need, we look at a condensate in a trap that makes it rotate about the z-axis, with only azimuthal movement. For the wave function of the condensate to be unique, we need a periodic function. The simplest form is then a wave function that varies as $e^{i \ell \varphi}$, where $\varphi$ is the azimuthal angle. Denoting the distance from the axis of the trap as $\rho$, the circulation equation gives

$$
\oint \boldsymbol{v} \cdot d \boldsymbol{l}=v_{\varphi} 2 \pi \rho=\frac{\hbar}{m} 2 \pi \ell
$$

which results in

$$
v_{\varphi}=\ell \frac{\hbar}{m \rho} .
$$

If there is a singularity inside the closed curve, $\ell \neq 0$, the condensate wave function must vanish at the axis of the trap $(\rho=0)$ to prevent the velocity, and thus the kinetic energy, from reaching infinity as $\rho \rightarrow 0$. This means that a singularity forming in the condensate must have the form of a vortex.

\subsubsection{The Energy of a Vortex}

The next question at hand is then: why or when do vortices form? The example above concerned a rotating condensate, and the present question is therefore a matter of energy minimization. When is it energetically favorable to form a vortex in a rotating condensate? The first thing we need to know is then the energy contribution of a single vortex. Based on equation (3.28), and the quantization integer $\ell$, the wave function may be written as

$$
\psi(\boldsymbol{r})=f(\rho, z) e^{i \ell \varphi},
$$

assuming that the trap has a symmetry axis, and where $\rho$ and $\varphi$ are as before. Inserting equation (3.44) into equation (3.27) results in the expression

$$
E=\int d \boldsymbol{r}\left\{\frac{\hbar^{2}}{2 m}\left[\left(\frac{\partial f}{\partial \rho}\right)^{2}+\left(\frac{\partial f}{\partial z}\right)^{2}\right]+\frac{\hbar^{2}}{2 m} \ell^{2} \frac{f^{2}}{\rho^{2}}+V(\rho, z) f^{2}+\frac{1}{2} U_{0} f^{4}\right\}
$$


describing the energy of the system, which consists of a rotating condensate with a vortex at the center of the rotational axis. The second term in equation (3.45) describes the rotational motion. An equation for the amplitude, $f$, can be found by inserting equation (3.44) into the time independent Gross-Pitaevskii equation (3.29), but this will not be stated here.

\section{Vortex in a Free Condensate}

The simplest situation would be the case where there is no trapping potential confining the condensate, and a condensate wave function not dependent on $z$. In addition, one can put $\ell=1$, that is, only look at a single quantum of circulation. Equation (3.45) describes the energy of the whole condensate system, but it is the energy contribution of the vortex that is of interest. This can be obtained by subtracting the energy of the system without any vortices, and the resulting vortex energy is

$$
\begin{aligned}
\epsilon_{\mathrm{v}} & =\int_{0}^{D} 2 \pi \rho d \rho\left[\frac{\hbar^{2}}{2 m}\left(\frac{d f}{d \rho}\right)^{2}+\frac{\hbar^{2}}{2 m} \frac{f^{2}}{\rho^{2}}+\frac{1}{2} U_{0}\left(f_{0}^{2}-f^{2}\right)^{2}\right] \\
& =\frac{\pi \hbar^{2}}{m} n \int_{0}^{D / \xi} x d x\left[\left(\frac{d \chi}{d x}\right)^{2}+\frac{\chi^{2}}{x^{2}}+\frac{1}{2}\left(1-\chi^{2}\right)^{2}\right] .
\end{aligned}
$$

From equation (3.35), $f$ is the number density and $f_{0}$ is the number density far away from the vortex. The variables $x=\rho / \xi$ and $\chi=f / f_{0}$ have been used to obtain the second line, where $\xi$ is the coherence length, describing the scale at which the wave function varies. $D$ is some finite distance from the vortex, as the integral would diverge otherwise. The coherence length also determines the size of the vortex core, as it is at this length scale the term in equation (3.45) responsible for the rotation dominates, and it is given as

$$
\frac{\hbar^{2}}{2 m \xi^{2}}=n U_{0}
$$

The solution of equation (3.46) is given as

$$
\epsilon_{\mathrm{v}} \approx \pi n \frac{\hbar^{2}}{m} \ln \left(1.464 \frac{D}{\xi}\right)
$$

where $n=f_{0}^{2}$.

\section{Vortex of Higher Circulation}

One of the simplifications in the calculation done above was putting $\ell=1$, that is, only considering one quantum of circulation. As it turns out, this is not a bad assumption. To logarithmic accuracy, the energy of a vortex with quantization $\ell$ is given as

$$
\epsilon_{\mathrm{v}} \approx \ell^{2} \pi n \frac{\hbar^{2}}{m} \ln \frac{D}{|\ell| \xi}
$$


Comparing this to equation (3.48) (to logarithmic accuracy), shows that the energy of a vortex with a higher quantization is larger than the energy of a collection of vortices with a single quantization with the same total circulation:

$$
\ell^{2} \pi n \frac{\hbar^{2}}{m} \ln \frac{D}{|\ell| \xi}>\pi n \frac{\hbar^{2}}{m} \ln \frac{D}{\xi}+\pi n \frac{\hbar^{2}}{m} \ln \frac{D}{\xi}+\pi n \frac{\hbar^{2}}{m} \ln \frac{D}{\xi}+\ldots
$$

In other words, it is energetically favorable to split a vortex of higher quantization into several vortices of a single quantization.

\section{Vortex in a Trapped Condensate}

As mentioned in the beginning of section 3.2, the most common trap potential in experiments is the harmonic oscillator potential, shown in equation (3.2). It is therefore of interest to also include the energy of a vortex in this kind of trap - a harmonic oscillator with a symmetry along the z-axis. As it turns out, we will need this result later in the thesis.

The calculations done in this case assumes the validity of the Thomas-Fermi approximation, which states, in simplified terms, that a high enough number of particles in the gas/fluid allows us to ignore the kinetic energy term in the Gross-Pitaevskii equation (3.29/3.30). Under this approximation, the chemical potential is large compared to the oscillator energy (see section 9.2.3 in [39]), resulting in the term describing the rotational motion to be dominant. This gives a result quite similar to the one in equation (3.48). What is different now is the finite extent of the gas/fluid as a result of the trapping potential. This will contribute to the vortex energy.

By just looking at the energy of the vortex out to some radius $\rho_{1}$, one obtains the same results as in equation (3.48). Now, however, the whole cloud has a finite radius, $\rho_{2}$, and thus the rest of the cloud will be affected by the vortex. The condensate velocity will be affected according to the value of the vortex circulation. In total, the energy of the vortex is given as

$$
\epsilon_{\mathrm{v}}=\pi n(0) \frac{\hbar^{2}}{m} \ln \left(1.464 \frac{\rho_{1}}{\xi_{0}}\right)+\frac{1}{2} \int_{\rho_{1}}^{\rho_{2}} m n(\rho) v^{2}(\rho) 2 \pi \rho d \rho,
$$

where the first term is the energy of the vortex itself, and the second term is the added kinetic energy of the condensate as a result of the vortex. Here $n(0)$ is the number density in the center in the absence of a vortex, and $\xi_{0}$ is the coherence length in the center, which for the harmonic oscillator case may be written as

$$
\xi_{0}=\frac{\hbar}{m \omega_{\perp} R} .
$$

Here $R$ is the radius of the cloud, and $\omega_{\perp}$ is the frequency of the trap for motion in the plane. Solving the integral in equation (3.51) gives the result:

$$
\begin{aligned}
\epsilon_{\mathrm{v}} & =\pi n(0) \frac{\hbar^{2}}{m}\left[\ln \left(1.464 \frac{\rho_{2}}{\xi_{0}}\right)-\frac{1}{2}\right] \\
& \simeq \pi n(0) \frac{\hbar^{2}}{m} \ln \left(0.888 \frac{\rho_{2}}{\xi_{0}}\right) .
\end{aligned}
$$


This has the same form as the free condensate vortex energy shown in equation (3.48) and is only valid as long as $\rho_{1} \ll \rho_{2}$.

Until this point, the problem has only been addressed in two dimensions. However, if the size of the fluid/gas cloud in the z-direction is a lot larger than the coherence length, the problem can be extended to three dimensions by adding together the horizontal slices of the cloud. This results in a total energy of the vortex state, compared to the ground state, given as

$$
E=\frac{4 \pi n(0,0)}{3} \frac{\hbar^{2}}{m} Z \ln \left(0.671 \frac{R}{\xi_{0}}\right) .
$$

Here $n(0,0)$ is the density in the center, in the absence of a vortex, $Z$ is the height of the cloud and $R$ is the radius of the cloud.

So far, all vortices have been located at the center of the condensate, that is, on the rotational axis. Off-axis vortices will have a slight adjustment to the energy, so that the energy of the vortex decreases as the distance from the axis increases. This indicates that vortices would tend to slide towards the edge of the cloud. However, the rotation of the trap itself can stabilize the vortex. See section 9.2.4 in [39] for more details on this.

\section{Vortex Formation}

It is now time to address the question asked in the beginning of section 3.3.2: why or when do vortices form?

Looking at an anisotropic trap without symmetries, a general criterion for the formation of vortices may be calculated. The trap rotates with an angular velocity of $\boldsymbol{\Omega}$ and in the rotating reference frame the energy of the cloud of atoms is given as

$$
E^{\prime}=E-\boldsymbol{\Omega} \cdot \boldsymbol{L},
$$

where $\boldsymbol{L}$ is the angular momentum and $E$ is the energy in the non-rotating frame. From equation (3.56) it can be shown that a state with angular momentum $\boldsymbol{L}$ along the rotational axis, with energy $E_{L}$, is energetically favorable to the ground state, if the angular velocity of the trap exceeds a critical value given by

$$
\Omega_{c}=\frac{E_{L}-E_{0}}{L} .
$$

Equation (3.55) shows the expression for the energy of the vortex state, compared to the ground state, in a three-dimensional HO trap. That means that equation (3.55) gives the value of the numerator in equation (3.57). The remaining quantity is then the angular momentum. Assuming the vortex to be at the rotational axis, the angular momentum will be quantized, and it can be calculated to have the value

$$
L=N \hbar=\hbar \int d \boldsymbol{r} n(\boldsymbol{r})=\frac{8 \pi}{15} n(0,0) R^{2} Z \hbar
$$


(see end of section 9.2.3 in [39]). Here, the symbols hold the same meaning as used previously in this subsection (3.3.2). The ratio between equation (3.55) and (3.58) gives the value of the critical angular velocity for the $\mathrm{HO}$ case:

$$
\Omega_{c}=\frac{5}{2} \frac{\hbar}{m R^{2}} \ln \left(0.671 \frac{R}{\xi_{0}}\right) .
$$

So, if the angular velocity of our gas/fluid, trapped in a harmonic oscillator potential, exceeds the value given in equation (3.59), vortex states will be favorable to the ground state. Thus, we now have the answer to when the formation of vortices may occur.

\section{Vortex Arrays}

The energy of a vortex of higher quantization was previously shown in equation (3.49), and it was concluded that vortices of higher quantization would split into several vortices of a single quantization. This then means that we can have a condensate containing several vortices. As the angular velocity of the cloud increases, one might go from one vortex to two, from two to three and so on, until a grid of vortices has formed. This will happen if the trap rotates at a velocity much larger than $\Omega_{c}$, and in general the grid will be triangular. To understand the movement of the condensate in this case, one might study the Hamiltonian in the rotating frame:

$$
\begin{aligned}
H^{\prime} & =H-\boldsymbol{\Omega} \cdot \boldsymbol{L} \\
& =\sum_{i=1}^{N}\left(\frac{\boldsymbol{p}_{i}^{2}}{2 m}+V\left(\boldsymbol{r}_{i}\right)-\boldsymbol{\Omega} \cdot\left(\boldsymbol{r}_{i} \times \boldsymbol{p}_{i}\right)\right)+U_{0} \sum_{i<j} \delta\left(\boldsymbol{r}_{i}-\boldsymbol{r}_{\boldsymbol{j}}\right) \\
& =\sum_{i=1}^{N}\left(\frac{\left(\boldsymbol{p}_{i}-m \boldsymbol{\Omega} \times \boldsymbol{r}_{i}\right)^{2}}{2 m}+V\left(\boldsymbol{r}_{i}\right)-\frac{m}{2}\left(\boldsymbol{\Omega} \times \boldsymbol{r}_{i}\right)^{2}\right)+U_{0} \sum_{i<j} \delta\left(\boldsymbol{r}_{i}-\boldsymbol{r}_{\boldsymbol{j}}\right) .
\end{aligned}
$$

In equation (3.61), the first term is the kinetic energy, the second term the external potential (trap), the third the rotational energy and the last term the two-body interaction energy. In equation (3.62), the first term is the kinetic energy in the rotating frame. In a classical system, this term may be made to vanish, thus minimizing the energy. This is done by putting the velocity of a particle equal to

$$
\boldsymbol{v}_{i}=\boldsymbol{p}_{i} / m=\boldsymbol{\Omega} \times \boldsymbol{r}_{i},
$$

corresponding to rotation as a rigid body. For this velocity, the curl will be a constant equal to $2 \Omega$. However, as shown in the beginning of section 3.3 , equation (3.37), the velocity field of a condensate can not take on this form, as it should be irrotational. Still, it is possible to form a velocity field that is approximately equal to that of a rigid body rotation. This can be done by creating a uniform grid of vortices aligned in the direction of the angular velocity. Indeed, this is what happens when the condensate rotates at velocities much larger than the critical one.

For the uniform grid of vortices, the average circulation is $2 \Omega$, provided that there is a high enough amount of vortices. The more vortices, the less the circulation will 
deviate from the rigid body case. For this to happen, the number of vortices per unit area in the plane perpendicular to $\Omega$ must equal

$$
n_{\mathrm{v}}=\frac{2 m \Omega}{h}=\frac{1}{\pi a_{\Omega}^{2}},
$$

where

$$
a_{\Omega}=\sqrt{\hbar / m \Omega}
$$

describes the intervortex spacing.

\subsection{Collective Excitations in the Condensate}

A Bose-Einstein condensate may exhibit collective excitations, known as phonons. The atoms in a substance are bound together, and as a result, if oscillations were to occur, an atom would not oscillate alone - the vibrations would travel through the whole material as a collective mode. In condensed matter physics, a phonon is a quantum mechanical description of elementary vibrational energy resulting from atoms oscillating at a single frequency in a solid or liquid. In the classical sense, oscillating atoms correspond to heat, and in a way, these collective modes transport heat through the substance. The collective modes have discrete energy levels, or quanta, which have been named phonons, much in the same way that a photon is a quantum of electromagnetic energy.

As the collective excitations travel through the material with some finite amount of energy, they may be treated as particles in quantum mechanics. For this reason, the phonons are often denoted as quasiparticles. For simple harmonic motion, two energy levels are separated by the energy quanta $\epsilon_{q}=\hbar \omega$, where $q$ is the wave number. In the low energy limit (for long wavelengths), the phonons may be interpreted as sound waves, and their propagating speed is the same as the speed of sound in the material. The energy is in this limit given as $\epsilon_{q} \approx c_{s} \hbar q$, where $c_{s}$ is the speed of sound.

In addition to phonons, there are many different modes that may arise in the condensate. These, together with the phonons, are covered in chapter 7 of [39], which thoroughly describes the dynamics of a condensate ${ }^{2}$.

\subsection{BEC vs. Superfluid}

In the introduction of this chapter, we mentioned that not all Bose-Einstein condensates are superfluids. As we have now described the BEC, under the assumption that it exhibits superfluid properties, we should explain what this entails.

Usually, a superfluid is characterized by its ability to flow without dissipation, that is, without loss of energy. In addition, a range of other properties lie within this

\footnotetext{
${ }^{2}$ Vortices are also regarded as excitations of the fluid. As we now know, they arise if the condensate is rotating and has therefore been given their own chapter in [39], namely chapter 9 .
} 
description, such as formation of quantized vortices, high heat conductivity and the ability to creep up along the walls of containers [45].

So, what makes a Bose-Einstein condensate exhibit superfluidity? To better understand this, we will consider a uniform Bose-Einstein condensate in the ground state, with a heavy obstacle moving through it. The obstacle moves with a constant velocity, $v$, and exerts a time-dependent potential on the particles in the condensate. Now, let us see this from a different perspective, where the obstacle is at rest and the condensate is flowing past it. If the energy and momentum of a system is $E$ and $\boldsymbol{p}$, the energy in a frame moving with velocity $\boldsymbol{v}$ is given by

$$
E(\boldsymbol{v})=E-\boldsymbol{p} \cdot \boldsymbol{v}+\frac{1}{2} M v^{2} .
$$

Here $M$ is the total mass of the system, and the expression results from a standard Galilean transformation. In the frame where the obstacle is at rest, the energy of the ground state is therefore

$$
E(\boldsymbol{v})=E_{0}+\frac{1}{2} M v^{2},
$$

as the momentum is zero in the frame where the condensate is at rest, and the groundstate energy is denoted by $E_{0}$ in the same frame. As previously mentioned, a superfluid flows without dissipation. One way of degrading the motion of the condensate is by creating excitations. Let us therefore consider a state with a single excitation of momentum $\boldsymbol{p}$. In the frame where the condensate is at rest, the energy is now given as

$$
E_{\text {ex }}=E_{0}+\epsilon_{p}
$$

where $\epsilon_{p}$ is the energy of the excitation. The energy in the frame where the obstacle is at rest is then

$$
E_{\mathrm{ex}}(\boldsymbol{v})=E_{0}+\epsilon_{p}-\boldsymbol{p} \cdot \boldsymbol{v}+\frac{1}{2} M v^{2} .
$$

By subtracting equation (3.67) from equation (3.69) we obtain the energy needed to create an excitation in the frame where the obstacle is at rest, $\epsilon_{p}-\boldsymbol{p} \cdot \boldsymbol{v}$. The advantage of considering this scenario in the frame where the obstacle is at rest is that we can consider its potential as static. This makes the obstacle unable to transfer energy to the condensate. We therefore have $\epsilon_{p}-\boldsymbol{p} \cdot \boldsymbol{v}=0$, which means that at a velocity

$$
v=\frac{\epsilon_{p}}{p},
$$

the obstacle can create an excitation in the condensate, with momentum parallell to $\boldsymbol{v}$. From this, excitations may be created at the minimal velocity of

$$
v_{c}=\min \left(\frac{\epsilon_{\mathrm{p}}}{\mathrm{p}}\right),
$$


referred to as the Landau critical velocity. For velocities lower than the Landau critical velocity, excitations are impossible to create, and the condensate will have superfluid properties. In addition, for velocities $v>v_{c}$, interactions between the excitations may increase the value of $v_{c}$ and prevent the superfluid phenomenon from ceasing.

Let us quickly look at an example. In a non-interacting Bose-Einstein condensate, the energy of a single excited particle is $\epsilon_{p}=p^{2} / 2 m$. This gives a velocity of $v=p / 2 m$, which means that $v_{c}$ is zero. This again means that, for all velocities, excitations can be created and the Bose-Einstein condensate can not exhibit superfluid properties. For a uniform Bose-Einstein condensate (with interacting bosons) ${ }^{3}, v$ is given as $v=c_{s} \sqrt{1+\left(p / 2 m c_{s}\right)^{2}}$, which means that $v_{c}=c_{s}$. This tells us that the lowest energy excitations are long-wavelength sound waves (phonons), and that the BEC is a superfluid below the speed of sound in the given fluid.

Now that we have the condensed matter physics background necessary to understand the condensate and the superfluid phenomenon, we can move on to study the superfluid dark matter model presented in [1].

\footnotetext{
${ }^{3}$ In this case the energy spectrum of excitations is given by the Bogoliubov expression, which for low momenta is $\epsilon_{p} \approx c_{s} p$. See section 8.1.2 and 10.1 in [39] for more details.
} 


\section{Chapter 4}

\section{Superfluid Dark Matter}

"You know, it seems to me there's so much more to the world than the average eye's allowed to see. I believe, if you look hard, there are more wonders in this Universe than you could ever have dreamed of."

- Van Gogh, Doctor Who: Vincent and The Doctor.

Superfluidity was first observed in liquid helium [46, 47, 48], and gained its name from its frictionless flow. Another characteristic of the superfluid is its response to rotation and the formation of quantized vortices. This topic was covered in chapter 3, section 3.3. The aforementioned chapter describes properties of the Bose-Einstein condensate in detail and is vital for our understanding of the superfluid behavior. It is now time to connect the known cold atom physics of BECs and superfluidity to the novel superfluid dark matter model introduced by Berezhiani and Khoury [1].

First, we briefly introduce the idea behind Modified Newtonian Dynamics (MOND) in section 4.1, to better understand why we so desire to reproduce the results yielded by MOND. In section 4.2 we explain the common two-fluid description used to characterize the superfluid and clarify how this results in the coexistence of the $\Lambda$ CDM model and a MONDian behavior at galactic scale. We then move on to introducing a quantum field theory approach to the superfluid description in section 4.3 , which will be convenient for future calculations.

\subsection{Properties of Modified Newtonian Dynamics (MOND)}

MOND was first proposed by Mordehai Milgrom in a paper from 1983 [6]. He presented how observational results in galaxies could be reproduced by modifying Newtonian dynamics, instead of assuming dark matter. The main result of this paper is given there as equation (1) and is now known as Milgrom's law:

$$
m_{g} \mu\left(\frac{a}{a_{0}}\right) \boldsymbol{a}=\boldsymbol{F} .
$$


Here $m_{g}$ is the gravitational mass of an object, $\boldsymbol{F}$ is a static force field and $\boldsymbol{a}$ is the acceleration of the object. The parameter $a_{0}$ is an acceleration constant, found to be

$$
a_{0} \approx 2 \times 10^{-8} \mathrm{~cm} \mathrm{~s}^{-2},
$$

which coincidentally is of the order $a_{0}^{2} \sim \Lambda$, in natural units (today's preferred value of the cosmological constant). The function $\mu\left(a / a_{0}\right)=\mu(x)$, for $x=a / a_{0}$, behaves as

$$
\mu(x \gg 1) \approx 1, \quad \mu(x \ll 1) \approx x,
$$

and so regular Newtonian dynamics is restored in the case where $a \gg a_{0}$. Summed up, the modified Newtonian dynamics presented by Milgrom in 1983 proposes a change to Newton's law of gravity for small accelerations, at the order of $a \ll a_{0}$. As Milgrom himself emphasizes, this is not a full theory, only an effective working formula, although it is able to reproduce a lot of observational results on galactic scales.

Milgrom himself set up three assumptions in [6], from which a theory could be built:

(a) "The Newtonian dynamics of a gravitating system (and perhaps of an arbitrary one) break down in the limit of small accelerations."

(b) "In this limit the acceleration, $a$, of a test particle is given by $a^{2} / a_{0} \approx g_{N}\left(g_{N}\right.$ being the conventional gravitational acceleration)."

(c) "The acceleration constant $a_{0}$ plays all the possible roles of such a constant in the modified dynamics (that of the proportionality factor as defined above, the transition acceleration from the Newtionian to the non-Newtionian regime and the width of the transition regime)."

There has been made several attempts at making full theories where modified Newtonian dynamics are included (see section 7 in [8]), but usually they require the addition of some sort of dark matter, or "dark fields," in the end. So why not just go with the well established $\Lambda$ CDM?

The intriguing part about MOND is its ability to predict galactic properties yet to be sufficiently explained by the cold dark matter model. A good review of all the problems faced by $\Lambda \mathrm{CDM}$ and how MOND might be a good solution is given in $[7,8]$. Still, we would like to mention a few of the biggest issues resolved by MOND, especially emphasized in [1] and [11]:

1. The Baryonic Tully-Fisher Relation [7, 8, 49]: The BTFR is a relation observed between the asymptotic/flat circular velocity, $v_{c}$, of a galaxy and the baryonic mass, $M_{b}$, within it:

$$
M_{b} \sim v_{c}^{4}
$$

The prediction regarding this kind of relation by $\Lambda \mathrm{CDM}$ and MOND is shown in figure 4.1, along with observational data (figure borrowed from $[7,8]$ ). The dashed line shows the $\Lambda \mathrm{CDM}$ prediction and the dotted shows that of MOND. Clearly, 
it is the modified Newtonian dynamics that best fit the observations, but this is not the main problem for $\Lambda \mathrm{CDM}$. The trouble arises from the low scattering at the high mass end of the slope. The baryonic feedback processes should result in larger scatter for different types of galaxies, and the tight correlation is somewhat hard to explain in the $\Lambda$ CDM model ([1] and internal sources). From MOND the correlation arises directly in the $a \rightarrow \sqrt{a_{0} g_{N}}$ regime by looking at the centripetal acceleration, which yields $a_{0} G M_{b}=v_{c}^{4}$ when equated to the effective acceleration, $a=\sqrt{a_{0} g_{N}}$.

2. Tidal dwarf galaxies [7, 8]: A Tidal Dwarf Galaxy (TDG) is a galaxy made out of the tidal remnants of a galaxy merger event. These galaxies are expected to be devoid of dark matter, as their creation process effectively separates the dark and baryonic matter. Some TDGs are, however, observed to follow the BTFR, and must thus contain as much dark matter as the other galaxies following this distribution. In the case where MOND replaces cold dark matter, however, in the weak acceleration limit, all systems should follow the BTFR, no matter when and how they were created.

3. The satellite phase-space correlation challenge [8]: $\Lambda \mathrm{CDM}$ predicts an isotropic or quasi-isotropic distribution of CDM subhalos around a Milky Way-type of galaxy. The observations of satellites around our own galaxy, however, are correlated in phase-space and lie within a planar structure. This distribution of satellites is in conflict with the $\Lambda \mathrm{CDM}$ predictions. One possible solution to this problem is that the satellites were formed tidally in an old merger event, and then in reality are old tidal dwarf galaxies. This would make the known "missing satellite problem" even worse in a $\Lambda$ CDM Universe, but might work in a MOND scenario (section 9.2 in $[8])$.

In addition to the three problems presented here, the acceleration constant, $a_{0}$, seems to appear in many other observed properties of galaxies (section 5.2 in [8]). Altogether, MOND seems like a good model at galactic scales, but as it so happens, Milgrom's law (4.1) does not work at larger scales, such as that for galaxy clusters $[9,51,52,53,54]$. There have been attempts at sculpting full theories which applies to all scales, but as mentioned before, dark matter (or very massive neutrinos) must usually somehow be added to bring it all together $[8,9]$. In addition, these theories have trouble reproducing the observed angular power spectrum of the CMB temperature fluctuations (section 9.2 in [8]). This contributes to the $\Lambda$ CDM model still being the most favored candidate as the standard model of Big Bang cosmology.

\subsection{Two-Fluid Description}

One of the most familiar and studied cases of superfluidity is that of liquid helium. Below a critical temperature, $T_{c}$, the liquid helium obtains certain properties, such as loss of friction, or in other words, flow without dissipation. The superfluid is thus a 


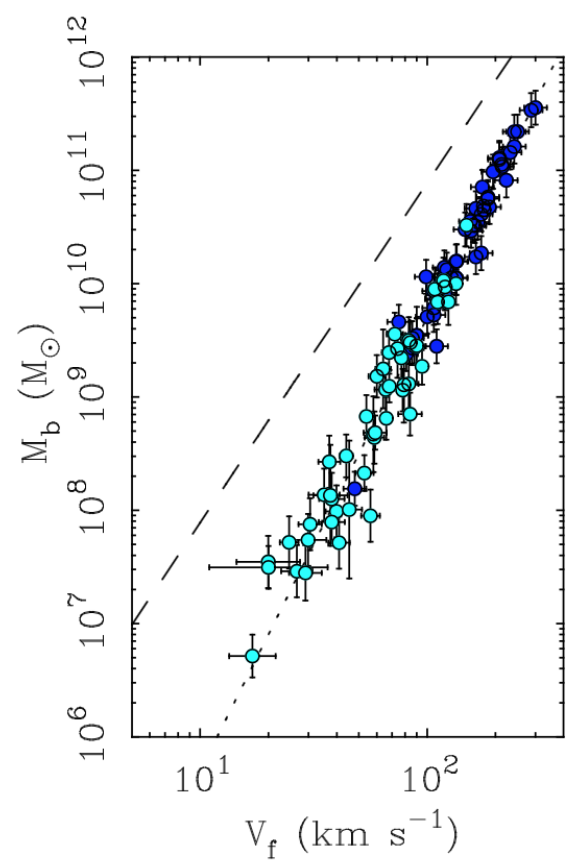

Figure 4.1: Baryonic Tully-Fisher Relation (BTFR). The BTFR is a generalization of the empirical Tully-Fisher relation presented in 1977 [50], and it states the relation $M_{b} \sim v_{c}^{4}$ for the circular velocity and baryonic mass of a galaxy. The baryonic mass consists of stars and gas, so that $M_{b}=M_{*}+M_{g}$. The light blue points in the figure are galaxies with $M_{*}<M_{g}$, while the dark blue points have $M_{*}>M_{g}$. The data points define the BTFR. The dotted line shows the predicted relation resulting from MOND, and the dashed line shows the prediction made by the $\Lambda$ CDM model, which is closer to $M_{b} \sim v_{c}^{3}$. In addition, $\Lambda \mathrm{CDM}$ predicts a larger scattering in the high mass end of the slope. The figure is borrowed from $[7,8]$.

phase of a given substance, obtained below this critical temperature. For liquid helium, this phase can be described through Bose-Einstein condensation, which today is well known in the field of low temperature physics (we again refer you to chapter 3 ).

One of the more common ways to extract information about the superfluid behavior is to use Landau's two-fluid description. Here the system is divided into two interpenetrating fluids, one behaving as a normal liquid, named the normal fluid, and the other similar to an ideal fluid, the superfluid (see chapter 10 in [39] and chapter 2 in [55]). The superfluid component consists of the Bose-Einstein condensate, and the normal component describes elementary excitations (particles in higher energy states than those making up the condensate). If the elementary excitations collide frequently enough to be in a local thermodynamical equilibrium, they may be described as a fluid, which is why this is called a two-fluid description. In a cloud of gas cooled down to its respective critical temperature (see section 3.2.1), it may not be the case that every single atom enters the superfluid. Only at zero temperature will we have the super- 
fluid alone. Gradually increasing the temperature from zero will introduce elementary excitations, and the normal component will become more and more prominent until it completely dominates at $T \geq T_{c}$. The two fluids describing the system have independent velocity fields and mass densities. For example, the momentum density of the system is described as

$$
\boldsymbol{k}=\rho_{s} \boldsymbol{v}_{s}+\rho_{n} \boldsymbol{v}_{n},
$$

where $s$ denotes the superfluid component and $n$ the normal component. The total mass density of the system is given as

$$
\rho=\rho_{s}+\rho_{n} .
$$

For very low temperatures, the superfluid density will approach the density of the liquid, completely dominating at zero temperature. Opposite, the density of the normal component will approach that of the fluid when the temperature rises towards $T_{c}$, and above this, the density of the superfluid component vanishes.

The two-fluid description is an important part of the superfluid dark matter model presented in [1]. Berezhiani and Khoury suggests a model where both MONDian and regular CDM behavior is a result of superfluid dark matter. This is obtained through different phases of the superfluid. Their model proposes that dark matter condenses and forms a superfluid inside, and around, the galactic disc, while the outer parts of the dark matter halo stays in the normal phase. This is illustrated in figure 5.1 from chapter 5, where the radius of the condensed part of the DM halo is estimated for a Milky Way-like galaxy. The fact that the dark matter only condenses into a superfluid in the inner regions of the halo is crucial. In the outermost parts of the halo, that is, in the normal component, the dark matter still behaves as the usual collisionless particles. In the inner parts of the halo, surrounding the whole galactic disk, the superfluid dark matter exhibits collective excitations, which we have encountered before as phonons (section 3.4). The idea presented in [1] is that the dark matter phonons are described by the same action as the non-relativistic MOND scalar action ${ }^{1}$,

$$
\mathscr{L} \sim \Lambda X \sqrt{|X|} .
$$

In addition, the phonons must couple to baryons in a certain way (see section 6.3), resulting in the phonons mediating a MONDian force between the ordinary matter in the galaxy. This way, they obtain regular $\Lambda$ CDM behavior at large scales, while reproducing the success of MOND at galactic scales. For this to be possible, the dark matter must be able to thermalize in the central regions. This requires the dark matter particles to be axion-like, quite light and repulsively interacting. It also requires a particular kind of superfluid which is dominated by three-body interactions, as opposed to two-body interactions (for full details see $[1,11]$ ).

Describing the dark matter in the central parts of the halo as a superfluid raises the question of vortices. As pointed out by Berezhiani and Khoury, a galaxy rotates

\footnotetext{
${ }^{1}$ See section 8.3 .1 for more information.
} 
fast enough that vortices should be formed in the fluid (assuming that the dark matter rotates together with the baryonic matter), and thus will be present throughout the galactic disk. The question is then whether these would have any impact on the behavior of the baryonic matter in the galaxy or not, and if so, is there any possibility of detecting this observationally? This is only briefly mentioned in the original paper proposing the idea, and it is the intention of this thesis to further investigate the properties of the vortices and their possible impact on the surroundings.

\subsection{Quantum Field Theory Approach}

To calculate the properties of the dark matter superfluid and its vortices, we need a sound theory in which to describe them. Imposing regular hydrodynamics with quantum mechanics, as has been done in chapter 3, is simply not realistic. The calculations are complicated and the vortices are way too small to be resolved in a galactic scale computer simulation (see chapter 5). After extensive research of the field, we have concluded that a field-theoretical approach offers the most beneficial description of the superfluid and its vortices for our purpose. This is because it enables us to use the well established Lagrangian formalism to study the vortices. This will be used in chapter 7 , when investigating the vortex-baryon interaction, and in chapter 8 , when investigating the Lagrangian density proposed for the model in [1]. This section is thus an introduction to the description of a superfluid in quantum field theory, and it will closely follow the approach made in [55].

As mentioned in the beginning of this chapter, superfluid helium is described as flowing without any friction. This happens when the helium is transported through a thin capillary [46, 48], and we may say that we move helium atoms, or equivalently just mass, through the capillary. Both of these quantities, mass and the number of atoms, are conserved in the system. In a relativistic system, however, mass is not in general conserved, but a system may have a conserved charge (or several), which in the case of the superfluid helium is the mass and number of atoms. In this situation, it might be said that superfluidity is transportation of a conserved charge, without any dissipation. We have earlier pointed out that the formation of quantized vortices (section 3.3) also is a property associated with superfluidity, and that the superfluidity often is connected to a Bose-Einstein condensate. In section 3.2.1 we explained how a BEC will occur below a critical temperature, $T_{c}$, which essentially tells us that a superfluid is a phase of the system which arise below a given threshold, individual to the system in question.

The phase transition resulting in a superfluid is in the field theory approach described through symmetries. Briefly explained, it arises when a global symmetry of the system, associated with one of these conserved charges mentioned above, is spontaneously broken. In more detail, for the case of a Bose-Einstein condensate, it occurs when the global U(1) symmetry of the system spontaneously breaks, as illustrated in figure 4.2. To describe this, using field theory, one constructs a Lagrangian characterizing the system in total. The system in our case being some bosonic gas/liquid with the possibility of obtaining a superfluid state. The Lagrangian will be symmetric under 

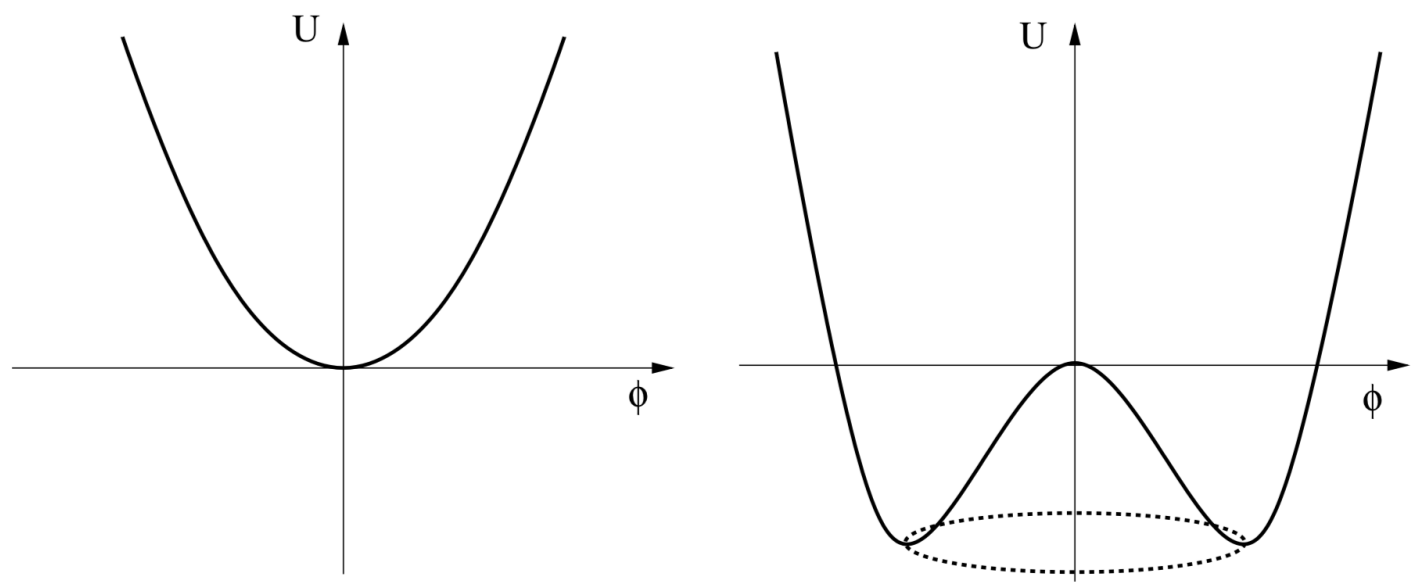

Figure 4.2: Illustration of the spontaneous symmetry breaking of the potential, $U(\phi)$, where $\phi$ is the condensate. The potential to the left has no broken symmetries, while the potential shown to the right has had a spontaneous symmetry breaking of the global $\mathrm{U}(1)$ symmetry. The circle at the bottom illustrates the phase, $\psi$, of the condensate and shows that there is no longer a trivial minimum point. The figure is borrowed from [55].

the $\mathrm{U}(1)$ symmetry, which is the simplest continuous rotational symmetry. We adopt the Lagrangian presented in equation (3.1) in [55],

$$
\mathscr{L}=\partial_{\mu} \phi^{*} \partial^{\mu} \phi-m^{2}|\phi|^{2}-\lambda|\phi|^{4},
$$

as it describes spin-0 bosons with mass $m$ repulsively interacting with a coupling constant $\lambda>0$. This is almost what we need to describe our superfluid dark matter, except for the fact that this Lagrangian contains two-body interactions instead of the three-body interactions we need to create our MONDian force. However, this is a good starting point for understanding the superfluid behavior in general, and we will therefore use it as a nice introductory problem. In equation (4.8), $\phi$ is a complex scalar field which depends on spacetime, $\phi=\phi(X)=\phi\left(x_{0}, \boldsymbol{x}\right)$, and $c=\hbar=1$. From this moment on, we will work in natural units, unless otherwise stated. We will also use capitalized letters for spacetime coordinates, and lowercase letters for regular spatial coordinates. The $\mathrm{U}(1)$ rotation is given as

$$
\phi \rightarrow e^{-i \alpha} \phi,
$$

which, seen by inserting into equation (4.8), leaves the Lagrangian invariant. As we are dealing with a global symmetry, $\alpha \in \mathbb{R}$ and does not depend on spacetime.

In section 4.2 we looked at the widely used two-fluid description of the superfluid and saw that it separated the condensate from the fluctuations. To incorporate the same effect here, the complex scalar field is split up into two parts,

$$
\phi(X) \rightarrow \Phi(X)=\phi(X)+\varphi(X) .
$$


Here $\phi(X)$ is the condensate, and $\varphi(X)$ the fluctuations. The condensate is a classical field, and its properties may be further investigated through the Euler-Lagrange equations. Based on equation (4.8), which just describes a system of interacting bosons, it is not evident that a Bose-Einstein condensate will be formed. This is only a result of a spontaneous symmetry breaking, which might happen at some point (we know it to be at the critical temperature). Dividing the complex scalar field in a condensate part and a part containing the fluctuations (4.10) is thus only a precaution at this point. For our purpose, which is describing the condensate, the fluctuations, $\varphi$, are not needed, and we will from now on only work with $\phi$, assuming $\varphi=0$. Further, we take use of equation (3.4) in [55] and write the condensate as a function of its modulus, $\rho_{S F}(X)$, and phase, $\psi(X)$ :

$$
\phi(X)=\frac{\rho_{S F}}{\sqrt{2}} e^{i \psi}
$$

which is on the same form as equation (3.28). Inserting this into equation (4.8) results in an expression for the Lagrangian, not containing any fluctuations:

$$
\begin{aligned}
\mathscr{L} & =\mathscr{L}^{(0)}+\text { fluctuations }, \\
\mathscr{L}^{(0)} & =\frac{1}{2} \partial_{\mu} \rho_{S F} \partial^{\mu} \rho_{S F}+\frac{1}{2} \rho_{S F}^{2}\left(\partial_{\mu} \psi \partial^{\mu} \psi-m^{2}\right)-\frac{1}{4} \lambda \rho_{S F}^{4} .
\end{aligned}
$$

From now on, we will just use $\mathscr{L}$ when referring to $\mathscr{L}^{(0)}$. The equations of motion for $\rho_{S F}$ and $\psi$ may then be calculated using the Euler-Lagrange equations,

$$
\begin{aligned}
\frac{\partial \mathscr{L}}{\partial \rho_{S F}}-\partial_{\mu} \frac{\partial \mathscr{L}}{\partial\left(\partial_{\mu} \rho_{S F}\right)} & =0, \\
\frac{\partial \mathscr{L}}{\partial \psi}-\partial_{\mu} \frac{\partial \mathscr{L}}{\partial\left(\partial_{\mu} \psi\right)} & =0,
\end{aligned}
$$

which from

$$
\begin{aligned}
\frac{\partial \mathscr{L}}{\partial \rho_{S F}} & =\rho_{S F}\left(\partial_{\mu} \psi \partial^{\mu} \psi-m^{2}\right)-\lambda \rho_{S F}^{3}, \\
\frac{\partial \mathscr{L}}{\partial\left(\partial_{\mu} \rho_{S F}\right)} & =\frac{\partial}{\partial\left(\partial_{\mu} \rho_{S F}\right)}\left(\frac{1}{2} \partial_{\mu} \rho_{S F} g^{\mu \nu} \partial_{\nu} \rho_{S F}\right) \\
& =\frac{1}{2} g^{\mu \nu}\left(\partial_{\nu} \rho_{S F}+\partial_{\mu} \rho_{S F} \delta_{\nu}^{\mu}\right) \\
& =\frac{1}{2} \partial^{\mu} \rho_{S F}+\frac{1}{2} \partial^{\nu} \rho_{S F} \delta_{\nu}^{\mu} \\
& =\partial^{\mu} \rho_{S F}, \\
\frac{\partial \mathscr{L}}{\partial \psi} & =0
\end{aligned}
$$




$$
\begin{aligned}
\frac{\partial \mathscr{L}}{\partial\left(\partial_{\mu} \psi\right)} & =\frac{\partial}{\partial\left(\partial_{\mu} \psi\right)}\left(\frac{1}{2} \rho_{S F}^{2}\left[\partial_{\mu} \psi g^{\mu \nu} \partial_{\nu} \psi-m^{2}\right]\right) \\
& =\frac{1}{2} \rho_{S F}^{2}\left(g^{\mu \nu} \partial_{\nu} \psi+g^{\mu \nu} \partial_{\mu} \psi \delta_{\nu}^{\mu}\right) \\
& =\frac{1}{2} \rho_{S F}^{2}\left(\partial^{\mu} \psi+\partial^{\nu} \psi \delta_{\nu}^{\mu}\right) \\
& =\rho_{S F}^{2} \partial^{\mu} \psi
\end{aligned}
$$

result in

$$
\begin{aligned}
\partial_{\mu} \partial^{\mu} \rho_{S F} & =\rho_{S F}\left(\partial_{\mu} \psi \partial^{\mu} \psi-m^{2}-\lambda \rho_{S F}^{2}\right) \\
\partial_{\mu}\left(\rho_{S F}^{2} \partial^{\mu} \psi\right) & =0 .
\end{aligned}
$$

Previously, we briefly mentioned that symmetries are associated with the conserved charges of the system. This is summed up in Noether's theorem, which states that for each continuous symmetry of the Lagrangian, there is a conserved quantity [56]. In more detail, equation (4.21) is the continuity equation for the conserved current, $j^{\mu}=\rho_{S F}^{2} \partial^{\mu} \psi$, of the system. This current has a corresponding conserved charge, $Q=\int d^{3} \boldsymbol{x} j^{0}$, which in our case is the number of particles. There is an additional symmetry (translational invariance) of the Lagrangian (4.8), which from Noether's theorem should also lead to a conserved quantity. In this case, the conserved quantity is the energy-stress tensor. The details of the calculation will not be summed up here, but can be found in [55], at the end of section 3.1, for those who might be interested.

In section 3.2 of the same paper, the role of the chemical potential, $\mu$, is presented. When considering a non-relativistic, non-interacting gas (see section 3.2.1 for our first encounter with this), the chemical potential is negative, and a BEC is obtained when $\mu=0$. In the relativistic regime, however, which is the case for us in the field theory approach, the chemical potential depends upon the rest mass of the particles, and a BEC is obtained when $|\mu|=m$. One may rewrite equation (4.20) as

$$
\partial_{\mu} \partial^{\mu} \rho_{S F}=\rho_{S F}\left(\sigma^{2}-m^{2}-\lambda \rho_{S F}^{2}\right)
$$

by introducing

$$
\sigma=\sqrt{\partial_{\mu} \psi \partial^{\mu} \psi} .
$$

By looking at the case where $\rho_{S F}$ and $\partial^{\mu} \psi$ are constant in space and time, one may deduce (section 3.2 in [55]) that $\sigma$ actually plays the role of the chemical potential in our system. It is also the case, in a U(1) gauge theory, that the chemical potential may be introduced directly in the Lagrangian or through the phase of the condensate, given as $\psi=\mu t$. Based on that argument,

$$
\mu=\partial_{0} \psi .
$$

This essentially leaves us with two chemical potentials, equation (4.23) and (4.24), which can only be the case if we put $\nabla \psi=0$. In reality, this is just two different points 
of view. In the rest frame of the superfluid, $\sigma$ plays the role of the chemical potential, while $\mu=\partial_{0} \psi$ is the case in the frame where the superfluid moves with a velocity decided by $\nabla \psi$. For more details on this in particular, please again consult [55]. We will take use of equation (4.24) when considering the chemical potential from here on out.

Another interesting consideration of this system is in relation to the Goldstone theorem. This states that every spontaneously broken continuous symmetry results in the appearance of a massless particle - a Goldstone boson (see section 11.1 in [57]). In the case of the $\mathrm{U}(1)$ symmetry break considered above, the resulting Goldstone boson is the phonon, previously explained in section 3.4, and needed to mediate the MONDian force through interaction with baryons in the superfluid DM model by Berezhiani and Khoury [1].

\subsubsection{The Vortex Solution}

From the equations of motion derived from the Lagrangian formalism, equation (4.20) and (4.21), we may recover the vortex equation describing the profile of a single straightline vortex. To do so, we look at a static situation so that $\rho_{S F}(X)=\rho_{S F}(\boldsymbol{x})$ and $\psi(X)=$ $\psi(\boldsymbol{x})$. We also have cylindrical symmetry, and may thus use cylindrical coordinates, $\boldsymbol{x}=(r, \theta, z)$, along with the properties

$$
\begin{aligned}
\nabla \psi & =\frac{\partial \psi}{\partial r} \vec{e}_{r}+\frac{1}{r} \frac{\partial \psi}{\partial \theta} \vec{e}_{\theta}+\frac{\partial \psi}{\partial z} \vec{e}_{z}, \\
\nabla^{2} \rho & =\frac{1}{r} \frac{\partial}{\partial r}\left(r \frac{\partial \rho}{\partial r}\right)+\frac{1}{r^{2}} \frac{\partial^{2} \rho}{\partial \theta^{2}}+\frac{\partial^{2} \rho}{\partial z^{2}} .
\end{aligned}
$$

We also assume that the condensate is independent of $z$, and that it is single-valued. This may be performed through an ansatz:

$$
\begin{gathered}
\psi=n \theta, \quad n \in \mathbb{N}, \\
\rho_{S F}(\boldsymbol{x})=\rho_{S F}(r),
\end{gathered}
$$

where $n$ is the winding number. For the condensate, $\phi$, being single valued means that it must return to its original value when moving along a circle, back to its original position. This, however, needs not be the case for the phase, and the winding number tells us how many times the phase is wound around the U(1) circle in the internal space, while the condensate moves around the circle in position space. Now, equation (4.21) follows directly from equation (4.25), (4.26), (4.27) and (4.28). The remaining is then equation (4.20), and to rewrite it we follow section 3.2 in [55] and introduce the variables

$$
R=\frac{\rho_{S F}}{\rho_{0}}, \quad \rho_{0}^{2}=\frac{\mu^{2}-m^{2}}{\lambda}, \quad \eta=\sqrt{\lambda} \rho_{0} r,
$$

where $\rho_{0}$ is the solution of equation (4.20) when $\nabla \rho_{S F}=\nabla \psi=0$, i.e. the homogeneous case. In addition, we use the same metric as stated in [55], namely $g_{\mu \nu}=$ 
$\operatorname{diag}(1,-1,-1,-1)$. Implementing all of this in equation (4.20) results in

$$
\begin{aligned}
& \partial_{\mu} \partial^{\mu} \rho_{S F}=\rho_{S F}\left(\partial_{\mu} \psi \partial^{\mu} \psi-m^{2}-\lambda \rho_{S F}^{2}\right) \\
&-\nabla^{2} \rho_{S F}=\rho_{S F}\left[\mu^{2}-(\nabla \psi)^{2}-m^{2}-\lambda \rho_{S F}^{2}\right] \\
&-\frac{1}{r} \frac{\partial}{\partial r}\left(r \frac{\partial \rho_{S F}}{\partial r}\right)=\rho_{S F}\left[\rho_{0}^{2} \lambda-\lambda \rho_{S F}^{2}-\left(\frac{n}{r}\right)^{2}\right] \\
&-\frac{1}{r} \frac{\partial}{\partial r}\left(r \frac{\partial \rho_{S F}}{\partial r}\right)=\rho_{S F}\left[\rho_{0}^{2} \lambda\left(1-\frac{\rho_{S F}^{2}}{\rho_{0}^{2}}\right)-\frac{n^{2}}{r^{2}}\right] \\
&-\frac{\sqrt{\lambda} \rho_{0}}{\eta} \frac{\partial}{\partial \eta} \frac{\partial \eta}{\partial r}\left[\frac{\eta}{\sqrt{\lambda} \rho_{0}} \frac{\partial \eta}{\partial r} \frac{\partial}{\partial \eta}\left(R \rho_{0}\right)\right]=R \rho_{0}\left[\frac{\eta^{2}}{r^{2}}\left(1-R^{2}\right)-\frac{n^{2}}{\eta^{2}} \lambda \rho_{0}^{2}\right] \\
&-\frac{1}{\eta} \frac{\partial}{\partial \eta}\left[\eta \frac{\partial R}{\partial \eta}\right]=R\left[1-R^{2}-\frac{n^{2}}{\eta^{2}}\right] \\
& \frac{1}{\eta} \frac{\partial}{\partial \eta}\left[\eta \frac{\partial R}{\partial \eta}\right]+\left(1-\frac{n^{2}}{\eta^{2}}\right) R-R^{3}=0 .
\end{aligned}
$$

Equation (4.36) may also be deduced through the Gross-Pitaevskii approach, and is shown as equation (9.12) in [39], using $n=1$. This is a good confirmation of the fact that the field theory approach reproduces the results obtained by the methods used in chapter 3. The solution of the vortex equation (4.36), found by the solve_bvp routine provided by SciPy [58], is shown in figure 4.3.

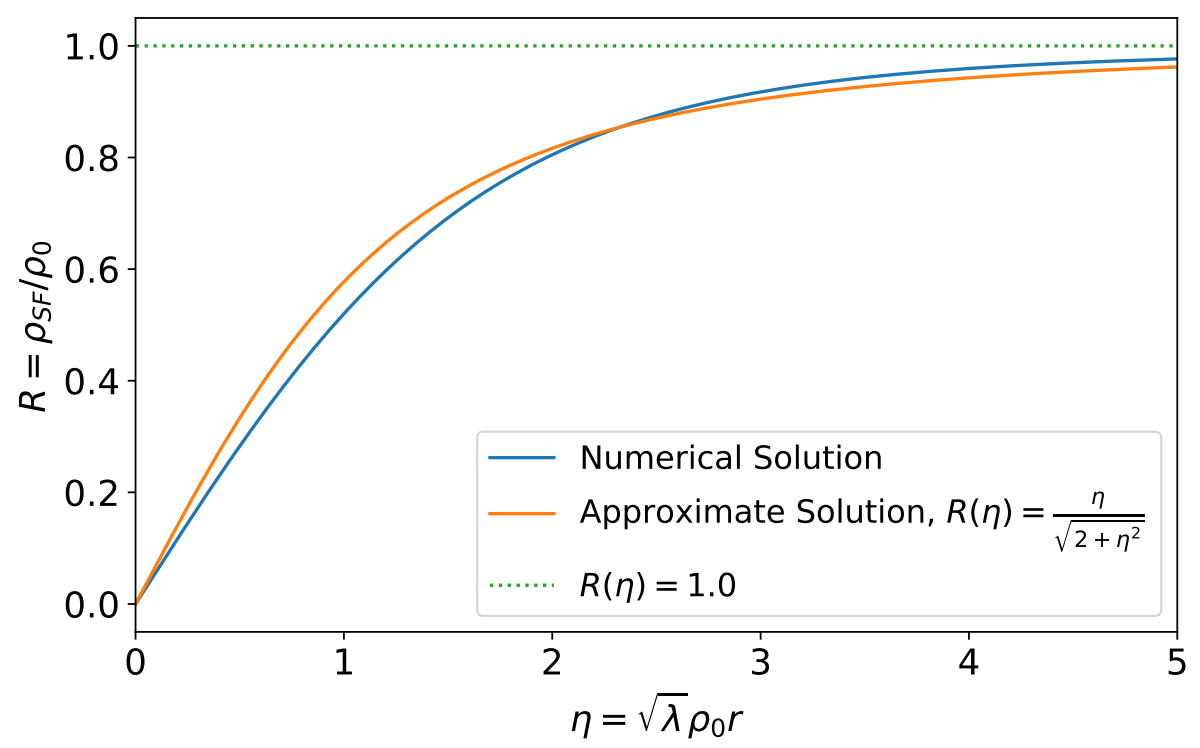

Figure 4.3: Profile of a single straight-line vortex. The blue line shows the numerical solution of equation (4.36), obtained using the boundary conditions $R(\eta=0)=0$ and $R(\eta \rightarrow \infty) \rightarrow 1$. The orange line shows an analytical expression close to the actual solution. We see that the condensate vanishes at the center of the vortex, which coincides with the theory presented in section 3.3.1. 
We have now introduced a field theory approach to our superfluid system and recovered the vortex solution. At this point we have all we need to perform further calculations concerning the vortices and their interactions with baryons, which after all is the main concern of this thesis. Before moving on to this, however, we need to get an overview of the properties of a typical vortex and how the distribution of vortices might look like in a galaxy such as the Milky Way. As the purpose of this is to provide a clearer mental picture of the situation at hand, and thus only meant as a guideline, chapter 5 will introduce a simplified model of a galaxy, and calculate a few resulting properties of the vortices. 


\section{Chapter 5}

\section{A Simple Model}

"Stop laughing. Are you all simple or something?"

- The Doctor, Doctor Who: Robot of Sherwood.

In this chapter we study vortices within a less complex galactic model, consisting of a spherical dark matter halo with constant density. The motivation behind this is to obtain a gravitational potential $\Phi \propto r^{2}$, where $r$ is the radius out from the center of the galaxy. In this way, we may apply the formulas derived for a harmonic oscillator potential, as presented in chapter 3 , to study our vortices.

We start out, in section 5.1, by demonstrating that a constant density indeed provides a $\Phi \propto r^{2}$ gravitational potential, and move on to applying the theory of chapter 3 to our vortex case in section 5.2. Finally, we take a look at the critical temperature of our constant density dark matter halo, in section 5.3.

\subsection{Gravitational Potential}

In local form, Newtons gravitational law can be written as

$$
\nabla^{2} \Phi=4 \pi G \rho
$$

where $\Phi$ is the gravitational potential, $G$ is the Newtonian gravitational constant and $\rho$ is the density. In our case, the latter will be constant. As a result of (assumed) spherical symmetry, the Laplacian can be expressed as

$$
\nabla^{2} \Phi=\frac{1}{r^{2}} \frac{\partial}{\partial r}\left(r^{2} \frac{\partial \Phi}{\partial r}\right)
$$


in spherical coordinates. The solution to equation (5.1) is then given as:

$$
\begin{aligned}
\nabla^{2} \Phi & =4 \pi G \rho \\
\frac{1}{r^{2}} \frac{\partial}{\partial r}\left(r^{2} \frac{\partial \Phi}{\partial r}\right) & =4 \pi G \rho \\
\int \mathrm{d}\left(\mathrm{r}^{2} \frac{\partial \Phi}{\partial \mathrm{r}}\right) & =\int 4 \pi G \rho r^{2} \mathrm{dr} \\
r^{2} \frac{\partial \Phi}{\partial r} & =\frac{4}{3} \pi G \rho r^{3}+\mathrm{C} \\
\int \mathrm{d} \Phi & =\int \frac{4}{3} \pi G \rho r \mathrm{dr}+\int \frac{\mathrm{C}}{\mathrm{r}^{2}} \mathrm{dr} \\
\Phi & =\frac{2}{3} \pi G \rho r^{2}-\frac{\mathrm{C}}{r}+\mathrm{D} .
\end{aligned}
$$

We require the potential to be well defined at $r=0$ and must therefore have $C=0$. This results in

$$
\Phi=\frac{2}{3} \pi G \rho r^{2}+D
$$

In other words, the gravitational potential in a sphere of constant density goes as $\propto r^{2}$. This means that we can use the results from chapter 9 in [39], describing Bose gas trapped in a harmonic oscillator (HO) potential, to characterize our spherical dark matter halo. All the information we need to do so is included in chapter 3, and the rest of this chapter will therefore follow these results, unless otherwise stated. The HO potential energy is given as

$$
V_{\mathrm{HO}}=\frac{1}{2} m \omega^{2} r^{2}
$$

whereas the gravitational potential energy, based on (5.9), is given as ${ }^{1}$

$$
V_{\Phi}=\frac{2}{3} \pi G \rho m r^{2}
$$

Here $m$ is the mass of the superfluid particle, which in this case is a DM particle. This results in the following relation between the density and angular frequency, $\omega$ :

$$
\rho=\frac{3}{4} \frac{\omega^{2}}{\pi G}
$$

or

$$
\omega=\sqrt{\frac{4 \pi G \rho}{3}} .
$$

From this, we may apply the equations of chapter 3 simply by replacing $\omega$ by equation (5.13). This will be done in the following section.

\footnotetext{
${ }^{1}$ For the simple purposes of this chapter we will ignore the constant $D$, or in other words, we will put the zero-point at the center of the sphere.
} 


\section{$5.2 \quad$ Vortex Properties}

Chapter 9 in [39] describes the behavior of vortices formed in a rotating superfluid, trapped in a HO potential. As a galaxy (and presumably the dark matter halo) rotates, vortices might form in the superfluid DM. This could have an impact on the surrounding baryonic matter, and might even lead to an observational criterion for this new DM model. In this section, we want to investigate some properties of such a vortex, to better understand what sort of situation we are dealing with. We will take a look at:

1. The energy of a vortex.

2. The coherence length of the superfluid.

3. The critical angular velocity.

4. The superfluid/condensate radius.

5. The intervortex spacing.

6. The "mass" of a single vortex.

All the equations needed to investigate this is given either in chapter 3 or in [11].

Let us start by stating and explaining some of the equations that we need. The energy per unit length of a vortex (in 2D) is given as

$$
\varepsilon_{\mathrm{v}} \simeq \pi n(0) \frac{\hbar^{2}}{m} \ln \left(0.888 \frac{R}{\xi_{0}}\right),
$$

for a Bose-Einstein condensate in a $\mathrm{HO}$ potential. The potential is assumed to be rotationally invariant about the $z$-axis and the vortex is centered at the rotational axis (see section 3.3.2 and equation 3.54). This equation is valid as long as there are enough particles that the Thomas-Fermi approximation holds, which we assume to be the case ${ }^{2}$. Here $n(0)$ is the number density, $\rho / m$, in the center of the condensate, in the absence of a vortex. Again $m$ is the DM particle mass, and $R$ is the radius of the condensate. Finally, $\xi_{0}$ is the coherence length of the superfluid, which for the HO trapped condensate is given as

$$
\xi_{0}=\frac{\hbar}{m \omega R}
$$

where $\omega$ is defined by equation (5.13), obtained in the previous section. The coherence length is important as it determines the size of the vortex core, as previously stated in section 3.3.2. In addition to the energy of a vortex and the coherence length, we want to look at the critical angular velocity of vortex formation. That is, which angular

\footnotetext{
${ }^{2}$ The Thomas-Fermi approximation was defined in section 3.3.2: Vortex In Trapped Condensate, and states that a high enough number of particles will allow us to ignore the kinetic energy term in the Gross-Pitaevskii equation, thereby simplifying the calculations.
} 
velocity must the superfluid rotate at for it to be energetically favorable to form a vortex? For a HO trap, this relation is given as:

$$
\Omega_{c}=\frac{5}{2} \frac{\hbar^{2}}{m R^{2}} \ln \left(0.671 \frac{R}{\xi_{0}}\right)
$$

where the variables are as described before (see section 3.3.2 and equation 3.59).

We now have the formulas needed to assess points 1-3 on our list, and we would like to look at some specific numbers. However, all the equations listed above need the value of the condensate radius to be calculated. In [11], the superfluid DM model for a galaxy is described as a multi-phased fluid (as explained in section 4.2). From the center of the galaxy and out to some radius, $R_{T}$, the dark matter is able to thermalize and condense into a superfluid. From some radius, $R_{\mathrm{NFW}}$, and out, the density profile of the DM halo follows a regular Navarro-Frenk-White (NFW) profile [59]. The two radii, $R_{T}$ and $R_{\mathrm{NFW}}$, have a small region where they overlap. This thermalization radius, $R_{T}$, is the one needed as radius input in our vortex equations. [11] gives the criterion

$$
R_{T} \lesssim 310 \mathrm{kpc}\left(\frac{\mathrm{m}}{\mathrm{eV}}\right)^{-8 / 7}\left(\frac{\mathrm{M}}{10^{12} \mathrm{M}_{\odot}}\right)^{1 / 7}\left(\frac{\sigma / \mathrm{m}}{\mathrm{cm}^{2} / \mathrm{g}}\right)^{2 / 7}
$$

for the superfluid radius in galaxy DM halos. Here $m$ is the DM particle mass, $M$ is the mass of the whole galaxy (baryonic and dark matter) and $\sigma / m$ is the self-interaction cross section per unit mass of the DM particles. It must be assumed that the DM particles self-interact within $R_{T}$ for them to be able to thermalize and condense. Outside $R_{\mathrm{NFW}}$, however, regular non-collisional DM is assumed. Based on [11] we will use

$$
m=1 \mathrm{eV} \quad \text { and } \quad \sigma / \mathrm{m}=0.01 \mathrm{~cm}^{2} / \mathrm{g} .
$$

With this we have the equations to asses points 1-4 on our list. There is now only one final missing quantity, the density $\rho$. For this we will use a mean density 200 times the present day critical density,

$$
\rho_{200}=200 \rho_{\text {crit }}=200 \frac{3 H_{0}^{2}}{8 \pi G} \simeq 1.84 \times 10^{-27} \mathrm{~g} / \mathrm{cm}^{3} .
$$

Here $H_{0}=70 \mathrm{kms}^{-1} \mathrm{Mpc}^{-1}$ and $G$ is the regular Newtonian gravitational constant. This mean density defines the virial radius, which is the radius within which the mean density is given as $200 \rho_{\text {crit }}$. From a galaxy formation perspective, this is the radius at which a collapsing overdensity has reached equilibrium. Equation (19) in [11] gives the virial radius as

$$
R_{200} \simeq 203\left(\frac{M}{10^{12} M_{\odot}}\right)^{1 / 3} \mathrm{kpc}
$$


At last we have what we need for points 1-4, and for a Milky Way-sized galaxy, with $M=10^{12} M_{\odot}$, we have the values

$$
\begin{array}{lll}
\text { 1. } & \varepsilon_{\mathrm{v}} & \simeq 1.135 \times 10^{-13} \mathrm{~g} \mathrm{~cm} \mathrm{~s}^{-2} \\
\text { 2. } & \xi_{0} & \simeq 0.1016 \mathrm{~cm} \\
\text { 3. } & R_{T} & \lesssim 83.16 \mathrm{kpc} \\
\text { 4. } & \Omega_{c} & \simeq 1.253 \times 10^{-39} \mathrm{~s}^{-1} .
\end{array}
$$

To put the condensate radius $\left(R_{T}\right)$ into perspective, the visible size of the Milky Way galaxy is only $\sim 30 \mathrm{kpc}$ in diameter [60], although the DM halo extends far beyond this. From equation (5.20), the virial radius of a Milky Way-sized galaxy is

$$
R_{200} \simeq 203 \mathrm{kpc} .
$$

It is important that the extent of the thermalization radius is larger than that of the stellar disk radius, as the superfluid dark matter model is able to describe the rotation curves of the galaxies and the baryonic Tully-Fisher relation with similar outcomes as MOND [11]. In doing so, the superfluid DM model reproduces observational results that have been troubling for the $\Lambda \mathrm{CDM}$ model (see section 4.1). The relative sizes between the galactic disk, the superfluid component and the full DM halo is roughly illustrated in figure 5.1.

So far, we are missing the last two points on the list presented in the beginning of this section, namely the intervortex spacing and the "mass" of a single vortex. Let us start with the intervortex spacing, and by noting that a typical angular velocity of a Milky Way-sized galaxy is

$$
\Omega_{\mathrm{MW}}=\frac{v}{r}=\frac{2 \pi r / T}{r}=\frac{2 \pi}{T}=\frac{2 \pi}{250 \mathrm{Myr}} \approx 8 \times 10^{-16} \mathrm{~s}^{-1} .
$$

This is much higher than the critical angular velocity (equation 5.24) for which the formation of vortices is energetically favorable. This shows that vortices should form easily in the rotating DM halo of the galaxy. As the angular velocity in a condensate is increased past the critical value, several vortices will form in a triangular pattern (assuming uniform rotation). These vortices all have a single quantum of circulation, as explained in section 3.3.2. Continuing to increase the angular velocity of the condensate will result in a large grid of vortices, spanning the whole condensate (still assuming uniform rotation $)^{3}$. Based on this we may then use the results from the end of section 3.3.2, which tell us that the number of vortices per unit area in the plane perpendicular to $\Omega$ is uniform, and equal to

$$
n_{\mathrm{v}}=\frac{2 m \Omega}{h}=\frac{1}{\pi a_{\Omega}^{2}} .
$$

\footnotetext{
${ }^{3}$ In the real world, however, the rotation of galaxies is not uniform. If this also is the case for the DM halo, it might lead to a different configuration of the vortex grid, as demonstrated by [61]. Here the vortices instead form a ring close to the edge of the condensate. This is, however, a problem for a more advanced assessment, and we will continue assuming a uniformly rotating condensate.
} 
$83 \mathrm{kpc}$

$203 \mathrm{kpc}$

Figure 5.1: Extent of the superfluid component compared to the size of the dark matter halo. The red line represents the radius of the galactic disk, the green line the radius of the superfluid dark matter component and the blue line the full extent of the dark matter halo. Beyond the superfluid radius the dark matter behaves as regular CDM particles, as in the $\Lambda \mathrm{CDM}$ model.

Here

$$
a_{\Omega}=\sqrt{\hbar / m \Omega}
$$

is a measure of the intervortex spacing (see equation 3.64 and 3.65). We then have all we need to calculate the next to final point on our list, and for a Milky Way-sized galaxy this results in an intervortex spacing of

$$
a_{\Omega} \sim 2.7 \times 10^{10} \mathrm{~cm} \approx 0.002 \mathrm{AU} .
$$

The last remaining point is then point number six, namely the "mass" of a single vortex. The energy we calculated in point one was taken from section 3.3.2, and it is the energy of a vortex in only two dimensions. The same section provides an expression for the vortex energy in three dimensions, for a single vortex sitting at the rotational 
axis of the system. In figure 5.2 we have tried to illustrate a single vortex at the center of a galaxy to better get an idea of what we are studying. Once we have the energy

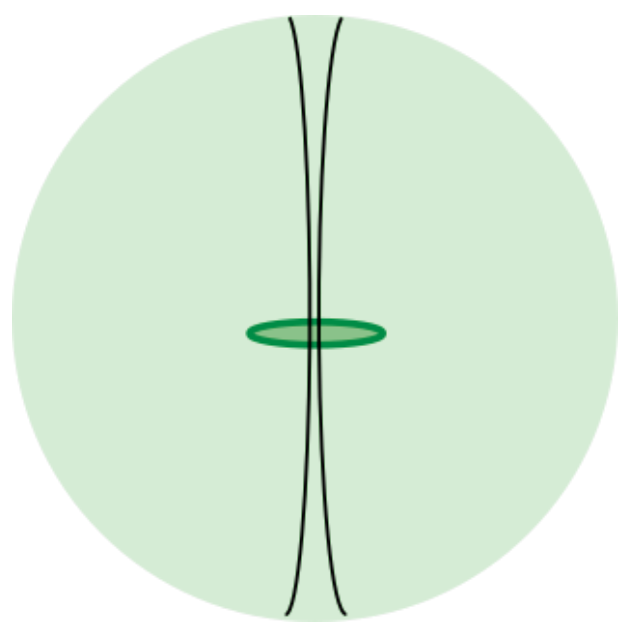

Figure 5.2: Condensate component with a single vortex at the galactic core. Here, the darker green disk illustrates the visible galactic disk and the lighter green circle the condensate component. The vortex profile is drawn in as black lines, although not in the correct size compared to the disk and condensate. In the illustration, the vortex core is wider towards the edge of the condensate. This will be explained in section 6.1.

in three dimensions, we may convert it to a mass to better envision its gravitational impact. First, let us find the energy. This is given by equation (3.55) in section 3.3.2 as

$$
E=\frac{4 \pi n(0,0)}{3} \frac{\hbar^{2}}{m} Z \ln \left(0.671 \frac{R}{\xi_{0}}\right),
$$

where $n(0,0)$ is the number density in the center in the absence of a vortex, $Z$ is the height of the condensate ${ }^{4}$ and $R$ is the radius of the condensate. Inserting the values from equations (5.19), (5.22) and (5.23) results in

$$
E \approx 3863 \mathrm{~J},
$$

which when converted to a mass equals

$$
m_{\text {vortex }} \sim 4 \times 10^{-14} \mathrm{~kg} .
$$

As we just calculated, the vortices are separated by $0.002 \mathrm{AU}$, and based on a $83 \mathrm{kpc}$ condensate radius, this gives a total of $\sim 10^{26}$ vortices within the condensate. Adding the mass of all the vortices together roughly amounts to the mass of ten million blue whales, which in itself is quite impressive, although utterly underwhelming on a galactic scale.

\footnotetext{
${ }^{4}$ The expression describes a cylindrical condensate, meaning that the resulting value might be slightly different for a sphere. The overall magnitude should, however, still be similar.
} 
Now that we have studied a few vortex properties for our simple galaxy model, it would also be interesting to address the critical temperature, which is an important property of the superfluid itself. This will be considered in the following section.

\subsection{Critical Temperature}

Until now, we have simply accepted that the dark matter has condensed and formed a superfluid. This is after all the main criterion of the model presented in [1]. Still, it would be interesting to calculate the critical temperature (see section 3.2.1) of the dark matter, along with the temperature that the dark matter will hold based on its self-interaction. Section 3.2.1 and equation (3.19) gives the critical temperature in a condensate trapped in a harmonic oscillator potential as

$$
T_{c} \approx 4.5\left(\frac{\bar{f}}{100 \mathrm{~Hz}}\right) N^{1 / 3} \mathrm{nK} .
$$

Here $N$ is the number of (dark matter) particles and

$$
\bar{f}=\bar{\omega} / 2 \pi, \quad \bar{\omega}=\left(\omega_{x} \omega_{y} \omega_{z}\right)^{1 / 3} .
$$

The number of dark matter particles may be calculated as the density ${ }^{5}$ (5.19) times the volume of the halo, divided by the mass (5.18) of a single particle. The volume is assumed to be spherical, and the full DM halo radius has been used $(R=203 \mathrm{kpc})$. This is done as we want to know which temperature this whole particle cloud must hold for it to (partly) condense. This results in

$$
N \approx 1.1 \times 10^{78}
$$

dark matter particles in the halo. Using the angular velocity stated in equation (5.13), together with equation (5.35) and (5.33) results in a critical temperature of

$$
T_{c} \approx 17 \mathrm{mK}
$$

for the DM halo.

When we look at dark matter as collisionless particles, talking about temperature might seem a bit strange. However, in this model the particles self-interact, and we may use the virial theorem $[63,64],\langle K\rangle=-\frac{1}{2}\langle U\rangle$, to translate gravitational energy into thermal energy. This relation between potential and kinetic energy is often written as

$$
\frac{3}{5} \frac{G M}{R}=\frac{3}{2} \frac{k_{B} T}{m}
$$

\footnotetext{
${ }^{5}$ The density is $200 \rho_{\text {crit }}$, where $\rho_{\text {crit }}$ is the critical matter density. This of course includes both dark and baryonic matter. However, in the Milky Way, about $90 \%$ of the mass is dark matter [62], and for this simple calculation we may take the given density as the dark matter density.
} 
where $M$ is the mass of the halo, $R$ is the radius, and $m$ is the mass of a single DM particle. $G$ and $k_{B}$ are as usual the gravitational constant and the Boltzmann constant. Inserting

$$
M=10^{12} M_{\odot}, \quad R=203 \mathrm{kpc} \quad \text { and } \quad \mathrm{m}=1 \mathrm{eV}
$$

we obtain

$$
T \approx 1.1 \mathrm{mK},
$$

showing that the dark matter has a temperature below the critical temperature inside the halo. As a remark, in [1], it it is not the temperature of the dark matter particles directly that is used to determine whether or not they can condense, but rather the de Broglie wavelength, $\lambda_{\mathrm{dB}} \sim 1 / m v$, of the particles. If the de Broglie wavelength of the particles overlap, we have superfluidity.

This final result concludes our investigation of vortex and condensate properties for a simple constant density dark matter halo. It is now time to look more closely at the proposed superfluid dark matter model and the parameters within it. This will be assessed in the next chapter. 


\title{
Chapter 6
}

\section{Parameter Variation}

\author{
"You know what the big problem is, in telling fantasy and reality apart? They're \\ both ridiculous."
}

- The Doctor, Doctor Who: Last Christmas.

In this chapter we want to take a further look at the parameters, $m$ and $\Lambda$, of the superfluid dark matter model, and whether or not there is anything that keeps us from changing them. The superfluid DM model is represented in $[1,11]$ as

$$
\mathscr{L}=\frac{2 \Lambda\left(2 m^{3 / 2}\right)}{3} X \sqrt{|X-\beta Y|}-\alpha \frac{\Lambda}{M_{\mathrm{Pl}}} \phi \rho_{b} .
$$

Here $X=\mu-m \Phi+\dot{\phi}-(\vec{\nabla} \phi)^{2} / 2 m$, where $\phi$ is the phonon scalar field and $\Phi$ is the gravitational potential. $Y$ is the scalar product of the normal and superfluid velocity fields, and $\beta$ parametrizes finite-temperature effects. We also have $M_{\mathrm{Pl}}$, the reduced Planck mass, $\alpha$, a coupling constant and $\rho_{b}$, the density profile of the baryons. The first term has the form of the MOND scalar action (see equation 4.7), while the second terms describes the interaction between phonons and baryons (see section 6.3 for more details). We are interested in the parameters, $m$, the dark matter particle mass, and $\Lambda$, the energy scale that defines the validity range of the model. In [1], $m=0.6 \mathrm{eV}$ and $\Lambda=0.2 \mathrm{meV}$ were used as fiducial values, whereas in [11] a new analysis was done regarding the condensate halo profile, and the fiducial values were chosen to be $m=1.0 \mathrm{eV}, \sigma / m=0.01 \mathrm{~cm}^{2} / \mathrm{g}$ and $\Lambda=0.05 \mathrm{meV}$ instead. In our calculations we will use the values $m=1.0 \mathrm{eV}, \sigma / m=0.01 \mathrm{~cm}^{2} / \mathrm{g}$ and $\Lambda=0.2 \mathrm{meV}$. This is to ensure that all constraints set up in [11] are fulfilled ${ }^{1}$, and also to ensure that the condensate radius is approximately the same in the two different approaches of [1] and [11].

We start off the chapter by calculating the zero-temperature density profile of the condensate (section 6.1), advancing from the constant density case of the previous

\footnotetext{
${ }^{1}$ In choosing $\Lambda=0.05 \mathrm{meV}$, the authors of [11] fail to fulfill their own constraint, as presented in their equation (28). The constraints do, however, allow for some wiggle room, which is why this particular one was not strictly implemented in the aforementioned paper. Still, we see no reason to not follow the equations as presented, and therefore choose to stay within the given boundaries.
} 
chapter. This enables us to see how the coherence length is affected by a non-constant density and inspires the work of section 6.2 , where we change our parameters to produce a larger vortex core. We further address an additional parameter, $\alpha$, in section 6.3, and investigate the effect of the parameter changes on the rotation curves of galaxies in section 6.4. We finish off by reducing the dark matter particle mass, exploring a lower limit of the parameter (section 6.5).

\subsection{Condensate Halo Density Profile}

Let us now look at the condensate halo density profile ${ }^{2}$. If we assume a static, spherically symmetric halo, the pressure and acceleration are related as

$$
\frac{1}{\rho(r)} \frac{d P}{d r}=-\frac{d \Phi}{d r}=-\frac{G m(r)}{r^{2}},
$$

where $\Phi=-G m / r$ is the regular gravitational potential field. We can write the mass $m(r)$ as

$$
m(r)=\int_{0}^{r} \int_{0}^{4 \pi} r^{2} \rho(r) d \Omega d r
$$

so that

$$
\frac{1}{\rho(r)} \frac{d P}{d r}=-\frac{G}{r^{2}} \int_{0}^{r} \int_{0}^{4 \pi} r^{2} \rho(r) d \Omega d r
$$

Going back to equation (6.2), we can now take the derivative with respect to $r$ and substitute equation (6.3) and (6.4):

$$
\begin{aligned}
\frac{d}{d r}\left(\frac{1}{\rho(r)} \frac{d P}{d r}\right) & =\frac{d}{d r}\left(-\frac{G m(r)}{r^{2}}\right) \\
& =\frac{d}{d r}\left(-\frac{G}{r^{2}} \int_{0}^{r} \int_{0}^{4 \pi} r^{2} \rho(r) d \Omega d r\right) \\
& =\frac{d}{d r}\left(-\frac{4 \pi G}{r^{2}} \int_{0}^{r} r^{2} \rho(r) d r\right) \\
& =\frac{8 \pi G}{r^{3}} \int_{0}^{r} r^{2} \rho(r) d r-\frac{4 \pi G}{r^{2}} \frac{d}{d r} \int_{0}^{r} r^{2} \rho(r) d r \\
& =-\frac{2}{r} \frac{1}{\rho(r)} \frac{d P}{d r}-4 \pi G \rho(r) .
\end{aligned}
$$

\footnotetext{
${ }^{2}$ This is based on section 3.2 of [1], and is derived for a superfluid at zero temperature, ignoring baryons. A more detailed calculation of the superfluid DM density profile is given in section V of [11].
} 
By moving the first term on the right-hand side over to the left-hand side, and multiplying by $r^{2}$, we obtain

$$
\begin{aligned}
r^{2} \frac{d}{d r}\left(\frac{1}{\rho(r)} \frac{d P}{d r}\right)+\frac{2 r}{\rho(r)} \frac{d P}{d r} & =-4 \pi G \rho(r) r^{2} \\
\frac{d}{d r}\left(r^{2} \frac{1}{\rho(r)} \frac{d P}{d r}\right) & =-4 \pi G \rho(r) r^{2} \\
\frac{1}{r^{2}} \frac{d}{d r}\left(r^{2} \frac{1}{\rho(r)} \frac{d P}{d r}\right) & =-4 \pi G \rho(r),
\end{aligned}
$$

which is the Poisson equation in spherical coordinates (with rotational symmetry). From equation (30) in [1], we may extract the equation of state for the superfluid dark matter $^{3}$ :

$$
P=\frac{\rho^{3}}{12 \Lambda^{2} m^{6}},
$$

which may be inserted into equation (6.12). Equation (6.12) may alto be written on a unitless form by first introducing the variable $y=\left(\rho / \rho_{\text {center }}\right)^{2}$, where $\rho_{\text {center }}$ is the central density of the halo. Writing equation (6.13) as

$$
P=K \rho^{3}
$$

and inserting the new variable leads to

$$
\frac{1}{r^{2}} \frac{d}{d r}\left(r^{2} \frac{d y}{d r}\right)=-\frac{8 \pi G}{3 K \rho_{\text {center }}} y^{1 / 2} .
$$

Further introducing $x=r / r_{0}$, where $r_{0}$ is some characteristic length, leads to

$$
\frac{1}{x^{2}} \frac{d}{d x}\left(x^{2} \frac{d y}{d x}\right)=-\frac{8 \pi G}{3 K \rho_{\text {center }}} r_{0}^{2} y^{1 / 2},
$$

where we may define

$$
r_{0}^{2}=\frac{3 K \rho_{\text {center }}}{8 \pi G}
$$

so that we have

$$
\frac{1}{x^{2}} \frac{d}{d x}\left(x^{2} \frac{d y}{d x}\right)=-y^{1 / 2} .
$$

Equation (6.18) is the Lane-Emden equation for $n=1 / 2$ [65], has the initial conditions $y(0)=1$ and $y^{\prime}(0)=0$, and may be solved numerically. Inserting the values of the different parameters results in the halo density profile of the condensate, which will give

\footnotetext{
${ }^{3}$ Notice that the equation of state has the form $P \propto \rho^{3}$, which implies that the superfluid DM interacts mainly through 3-body interactions. This is a result of requiring that the superfluid DM phonons are described by the MOND scalar action. See section 1.3 and 3.1 in [1] for a more detailed description.
} 
us the condensate radius. All of the constants are well know except for $\Lambda$ and $\rho_{\text {center }}$, which we may find in $[1,11]$. Equation (44) in [1] gives the central density as

$$
\rho_{\text {center }} \simeq\left(\frac{M}{10^{12} M_{\odot}}\right)^{2 / 5}\left(\frac{m}{\mathrm{eV}}\right)^{18 / 5}\left(\frac{\Lambda}{\mathrm{meV}}\right)^{6 / 5} 10^{-24} \mathrm{~g} / \mathrm{cm}^{3},
$$

where we use $M=10^{12} M_{\odot}$ and $m=1 \mathrm{eV}$ as before. Our value for $\Lambda$ was specified in the introduction of the chapter, but may also be implied by a constraint presented in equation (28) of [11]:

$$
\Lambda m^{3} \gtrsim 0.08 \mathrm{meV} \times \mathrm{eV}^{3} .
$$

This constraint is a result of demanding that the superfluid DM model allows for formation of the lightest observed halos, such as ultra-faint dwarf satellites. For our value of $m=1 \mathrm{eV}$, we get $\Lambda \gtrsim 0.08 \mathrm{meV}$. We will choose $\Lambda=0.2 \mathrm{meV}$ (as mentioned earlier), which leaves us with the following parameters for the model:

$$
m=1 \mathrm{eV}, \quad \sigma / \mathrm{m}=0.01 \mathrm{~cm}^{2} / \mathrm{g} \quad \text { and } \Lambda=0.2 \mathrm{meV} .
$$

We now have all the values that we need, and the resulting condensate DM density profile can be seen in figure 6.1 .

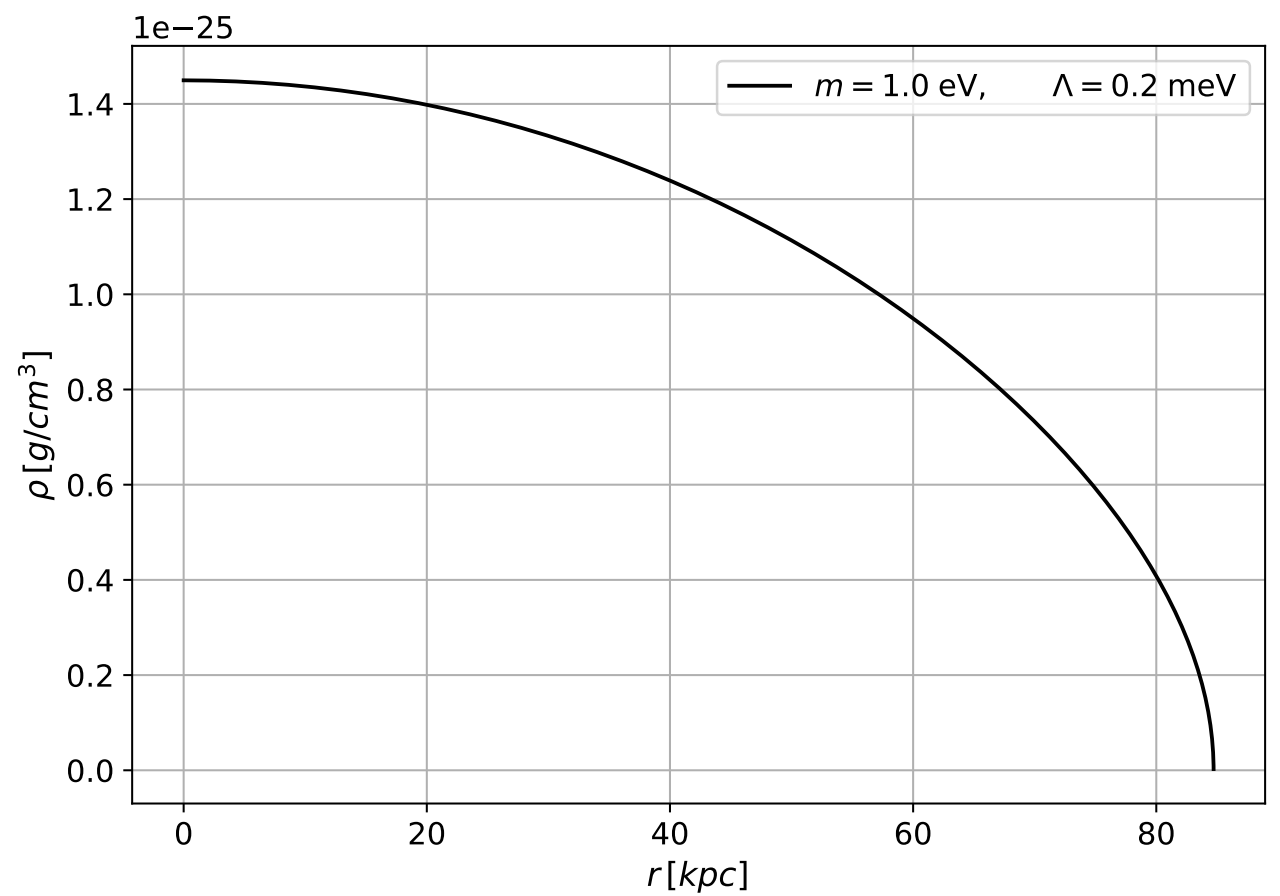

Figure 6.1: Zero-temperature condensate dark matter density profile, ignoring baryons. The parameters presented in equation (6.21) have been used. Defining the condensate radius as the point where the density profile reaches zero, we obtain a condensate radius of $\sim 84.7 \mathrm{kpc}$. 
The condensate density reaches zero at approximately $84.7 \mathrm{kpc}$, which we will use to define our condensate radius ${ }^{4}$. This fits the demand that $R_{T}>60 \mathrm{kpc}$ for a Milky way-sized galaxy (equation (23) in [11]), which is imposed as we want the superfluid $\mathrm{DM}$ to be able to explain rotation curves, typically measured out to $\sim 60 \mathrm{kpc}$ for a $10^{12} M_{\odot}$ Milky Way-like galaxy.

\subsubsection{Vortex Core Size}

In chapter 5 we calculated vortex properties based on a constant dark matter density profile. One of the quantities we looked at was the coherence length of the condensate, or in other words, the vortex core size. The equation describing this quantity (equation 5.15 ) depends upon the variable $\omega$, which again depends upon the density as

$$
\omega=\sqrt{\frac{4 \pi G \rho}{3}}
$$

One immediate consequence of our now non-constant density profile is that the vortex core size will vary throughout the condensate. It is therefore interesting to check how the coherence length behaves as a function of the density. As the coherence length also defines the size of the vortex core, this will show us how the size of the vortex core varies throughout the superfluid DM component. A plot illustrating this is presented in figure 6.2. Here it can be seen that the coherence length (vortex core) is at its smallest near the center of the condensate, and increases towards the edge, as illustrated in figure 5.2. The size is, however, never larger than the millimeter range, when using the parameters in equation (6.21). More precisely, the vortex core size varies between the minimal value in the center and the maximum value at the edge as given below:

$$
\xi_{\text {center }}=1.1 \times 10^{-4} \mathrm{~m}, \quad \xi_{\text {edge }}=2.7 \times 10^{-3} \mathrm{~m} .
$$

For this calculation we have used the expression for the coherence length given in equation (5.15). This depends upon the angular frequency of the condensate (equation 5.13), which again depends upon the density. This expression originates from a condensate trapped in a $\mathrm{HO}$ potential, which we imitated in the previous chapter by using a constant halo density. This is, however, not the case anymore, as the condensate density varies throughout the superfluid core. Still, on vortex scales, the density may safely be assumed to stay constant, and we can continue using equation (5.15).

\footnotetext{
${ }^{4}$ This is close to the constraint $R_{T} \lesssim 83 \mathrm{kpc}$ found in chapter 5 , however, it does not have to be. Defining the condensate radius by where the condensate density hits zero was done in [1], but in [11] a new assessment of the dark matter density profile was performed, which led to a new definition of the condensate, namely the thermalization radius, $R_{T}$, used in the previous section. This new thermalization radius is in general lower than the radius obtained from the zero temperature profile, depending on the choice of $\Lambda$. This is why we have chosen $\Lambda=0.2 \mathrm{meV}$. The given value ensures that the radius obtained by the methods in this section is close to the upper bound on the radius obtained from the $R_{T}$ expression. In the future, we will therefore assume that the two radii are the same.
} 


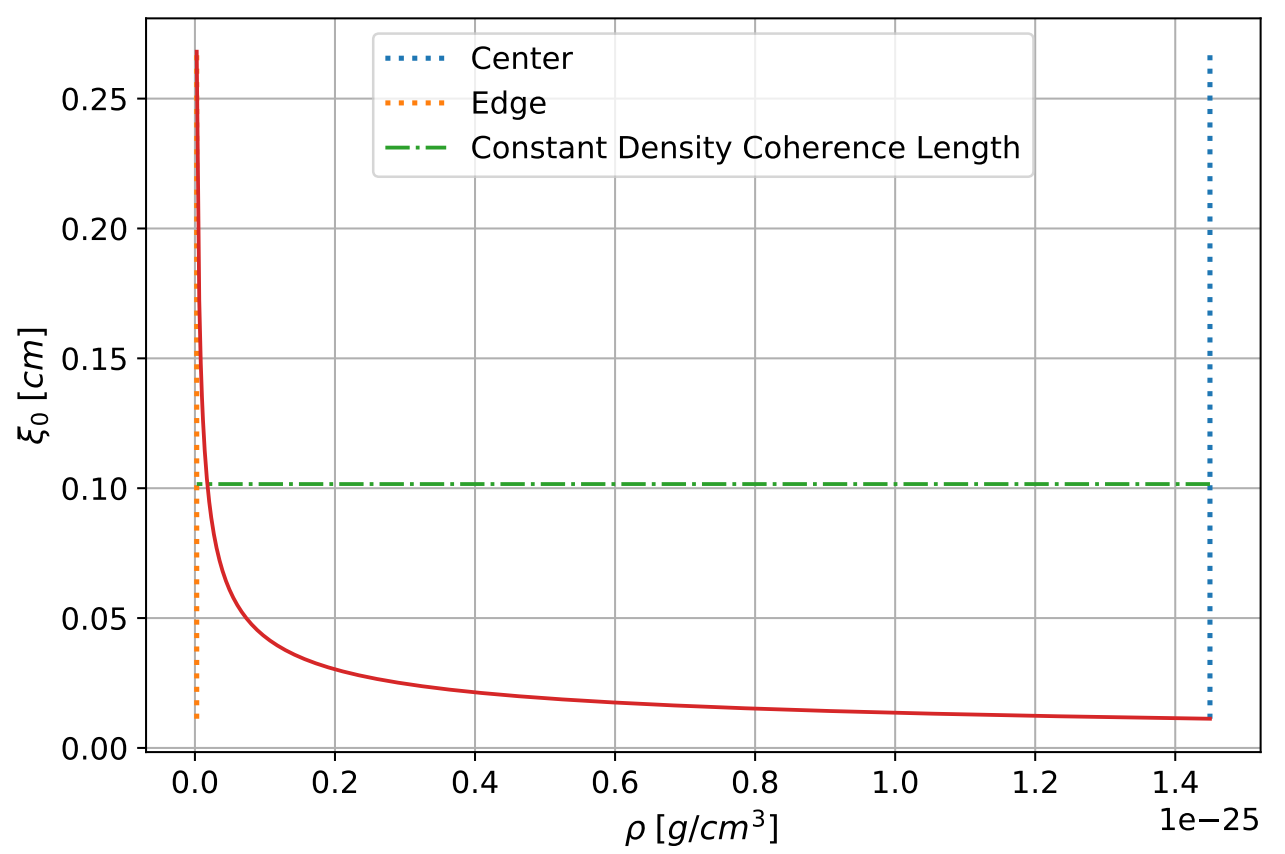

Figure 6.2: Coherence length behavior throughout the superfluid DM component, showing how the size of the vortex core changes as a function of density. The dashed/dotted green line marks the coherence length resulting from the constant density case, presented in section 5.2 , using $R_{T} \approx 83 \mathrm{kpc}$. The orange and blue dotted lines mark the edge and center of the condensate component.

In the vast space covered by the whole condensate component, a millimeter-sized vortex is tiny. From the previous chapter, the vortices are separated by $0.002 \mathrm{AU}$, and based on a $85 \mathrm{kpc}$ condensate radius, this would amount to roughly $10^{26}$ vortices within the condensate. Even though they are many, they are small, which makes any noticeable impact on the surrounding baryons sound improbable. This was also very apparent when calculating the "mass" of a single vortex in section 5.2, equation (5.32). Based on this, we might wonder whether or not a larger vortex core would result in a greater impact on the surroundings. From the energy expression in equation (5.30), it looks like a larger vortex core should result in a lower energy per vortex. However, to obtain a larger core we need a smaller DM particle mass (see equation 5.15), which from the energy expression should lead to larger energy values. As the coherence length appears inside a logarithm, while the mass appears outside, it should be the case that a smaller DM mass, and thus larger vortex core, all together results in a higher energy per vortex. This will be further investigated in the section below. 


\subsection{One Meter Vortex Core}

As we have just seen, the vortex core size varies from $1.1 \times 10^{-4} \mathrm{~m}$ at the center of a Milky Way-like galaxy, to $2.7 \times 10^{-3} \mathrm{~m}$ at the edge. These values are incredibly small in comparison to the great spatial extent of a galaxy, and the vortices would presumably have a small, if not negligible, impact on any surrounding baryons. From the argument given at the very end of the previous section, it seems like a smaller DM particle mass, and thus a larger vortex core, could be the key to obtaining high enough vortex energies to leave an imprint on the baryonic matter in the galaxy. This is interesting from an observational point of view, as an additional gravitational effect on baryons, from vortices, might be measurable. If that was the case, it would be helpful in distinguishing the superfluid DM model from other models, and at the same time limit the superfluid DM particle mass. However, if such an effect is not observable, it does not rule out the model. If the suspicions motivating the investigation presented in this section is correct, it would only tell us that the particle mass is too high for the vortex energy to be prominent. Still, an investigation is due, and it will be focused on changing the parameters of the model to create a larger vortex core.

Based on equation (5.15), giving the vortex core size as $\xi_{0}=\frac{\hbar}{m \omega R}$, it is clear that a reduction of the dark matter particle mass would increase the core size, at least if we could keep the density profile, and thereby the condensate radius, constant. Based on equation (5.30), a lower DM particle mass should increase the energy per vortex, along with increasing the vortex core size. Our condensate profile depends upon $\rho_{\text {center }}$, which again depends upon $m, \Lambda$ and $M . M$ is the total mass of the galaxy, which we will take as a constant. If we want to change the vortex core size by decreasing the particle mass $m$, we must therefore check if there are any values of $\Lambda$ which will allow us to obtain the same density profile as before. In this section we will have to go through a number of checkpoints in order to ensure that changing the parameters does not violate any constraints set up for the model in [11]. Some of these points require some extra attention and have therefore been given their own sections, while the rest of them will be investigated here. The checkpoints are as follows:

1. The Particle Mass: Does the model allow a decrease of the DM particle mass, $m$ ?

2. The $\Lambda$-value: Can we find a $\Lambda$-value that reproduces the original density profile? Does it still fulfill the constraints presented in [11]?

3. The Self-Interaction: How does changing $m$ and $\Lambda$ affect the self-interaction cross section? Does it still fulfill the constraints presented in [11]?

4. The Temperature: Is the halo temperature still below the critical temperature?

5. The Critical Angular Velocity: Is the angular velocity of the galaxy still higher than the critical angular velocity?

6. The Energy of a Single Vortex: Are we actually achieving a larger energy/mass per vortex, as expected? 
7. The $\alpha$ Parameter: There is another parameter of the model, $\alpha$, yet unmentioned. Does this additional parameter affect our parameter changes? See section 6.3.

8. Rotation Curves: Do the parameter changes affect the ability of the model to reproduce galactic rotation curves? See section 6.4.

In order to neatly go through the first six points on the list, we will choose a specific vortex core size and check whether or not the parameter changes permit us to stay within the model constraints. As the section title reveals, we will try to produce a one meter vortex core. The equation describing the vortex core size depends upon the density profile, which makes it clear that a mass that results in a one meter vortex core at the center does not result in a one meter vortex core at the edge, and vice versa. We will therefore consider both cases.

\section{The Particle Mass}

As the vortex core size is $1 \mathrm{~m}$, an incredibly small distance on galactic scales, we may assume the dark matter density to be approximately constant at the given length scales. Based on this, we may use the following expressions from section 5.2, used to describe a constant density halo:

$$
\xi_{0}=\frac{\hbar}{m \omega R} \text { and } \omega=\sqrt{\frac{4 \pi \mathrm{G} \rho}{3}} .
$$

Rearranging these, we get the expression

$$
m=\frac{\sqrt{3} \hbar}{\xi_{0} \sqrt{4 \pi G \rho} R}
$$

for the DM particle mass. Inserting the condensate radius $(84.7 \mathrm{kpc})$, the density values at the center or edge of the condensate, and $\xi_{0}=1 \mathrm{~m}$, we arrive at the two masses required to produce a one meter vortex core at the center/edge of the condensate:

$$
m_{\text {center }} \approx 1.1 \times 10^{-4} \mathrm{eV} \text { and } \mathrm{m}_{\text {edge }} \approx 2.7 \times 10^{-3} \mathrm{eV} .
$$

Here $m_{\text {center }}$ denotes the required mass to produce a one meter vortex core at the center of the condensate, while $m_{\text {edge }}$ denotes the required mass to produce a one meter vortex core at the edge. In other words, obtaining a one meter vortex core in the center requires a mass of approximately $\sim 10^{-4} \mathrm{eV}$. This would result in even larger vortices at the edge (approximately $\sim 24 \mathrm{~m}$ ). To obtain one meter vortex cores at the edge, however, we need a mass of meV scale. This would give a center vortex core of roughly $4 \mathrm{~cm}$. The general consensus is therefore, the lower the DM particle mass, the larger vortices we may obtain.

We now know which masses we need to produce the desired vortex cores, and we must move on to the question posed in checkpoint one: does the model allow us to change the particle mass? In the previous chapter, we mentioned that the condensate 
radius should extend beyond $60 \mathrm{kpc}$ for a Milky Way-sized galaxy. This is to ensure that the superfluid model can explain the observed flat rotation curves of galaxies. This demand results in an upper bound on the DM particle mass, given as equation (24) in [11]:

$$
m \lesssim 4.2\left(\frac{\sigma / m}{\mathrm{~cm}^{2} / \mathrm{g}}\right)^{1 / 4} \mathrm{eV} .
$$

For our value of $\sigma / m=0.01 \mathrm{~cm}^{2} / \mathrm{g}$, this results in $m \lesssim 1.33 \mathrm{eV}$, which fits our original value of $1 \mathrm{eV}$. As this is an upper bound it does not, at first sight, rule out the one meter vortex core masses in equation (6.26). However, changing both $m$ and $\Lambda$ might result in changes of $\sigma / m$, and this point will therefore have to be revisited at a later time.

\section{The $\Lambda$-value}

As nothing so far seems to prohibit us from operating with lower masses than suggested in [11], we may move on to checkpoint two: which value must $\Lambda$ take in each case for the halo profile in section 6.1 (figure 6.1 ) to be replicated? To obtain some approximate values for this, we again follow the procedure of section 6.1 , and solve the density profile numerically. After obtaining the general profile, we may insert the values of the constants, and retrieve the actual profile with the correct units and values. This time we use the new mass-values, which we calculated in the previous checkpoint. However, the value of $\Lambda$ is now unknown, and we must make a guess. This is done by choosing several values of $\Lambda$, of vastly different magnitudes, and then checking how far from the original profile the new profiles are. If they are within one order of magnitude, we keep the $\Lambda$ guesses. From that point we take the minimal and maximal $\Lambda$-value and make new guesses which lie in between. In that way we obtain density profiles that closely match the original one. A plot showing a few different values of $\Lambda$, together with the original density profile, is shown in figure 6.3 for the two different masses.

From figure 6.3 it is evident that there does exist $\Lambda$-values that will, in combination with the new particle masses presented in equation (6.26), reproduce the density profile obtained using the original values: $m=1 \mathrm{eV}, \sigma / m=0.01 \mathrm{~cm}^{2} / \mathrm{g}$ and $\Lambda=0.2 \mathrm{meV}$. The required $\Lambda$-values are

$$
\Lambda_{\text {center }} \approx 141 \mathrm{MeV} \text { and } \Lambda_{\text {edge }} \approx 10.4 \mathrm{keV} .
$$

We have now answered the first of the two questions posed in checkpoint two, and we may now address the second: do the new $\Lambda$-values still fulfill the constraints presented in [11]? For this we will revisit the constraint presented in equation (6.20), namely $\Lambda m^{3} \gtrsim 0.08 \mathrm{meV} \times \mathrm{eV}^{3}$. Inserting the values of $m$ given in equation (6.26) results in

$$
\Lambda_{\text {center }} \gtrsim 56 \mathrm{MeV} \text { and } \Lambda_{\text {edge }} \gtrsim 4 \mathrm{keV},
$$

which equation (6.28) fulfills. So far it therefore seems like we may reduce the original mass scale of the superfluid DM model and still reproduce the original density profile. 


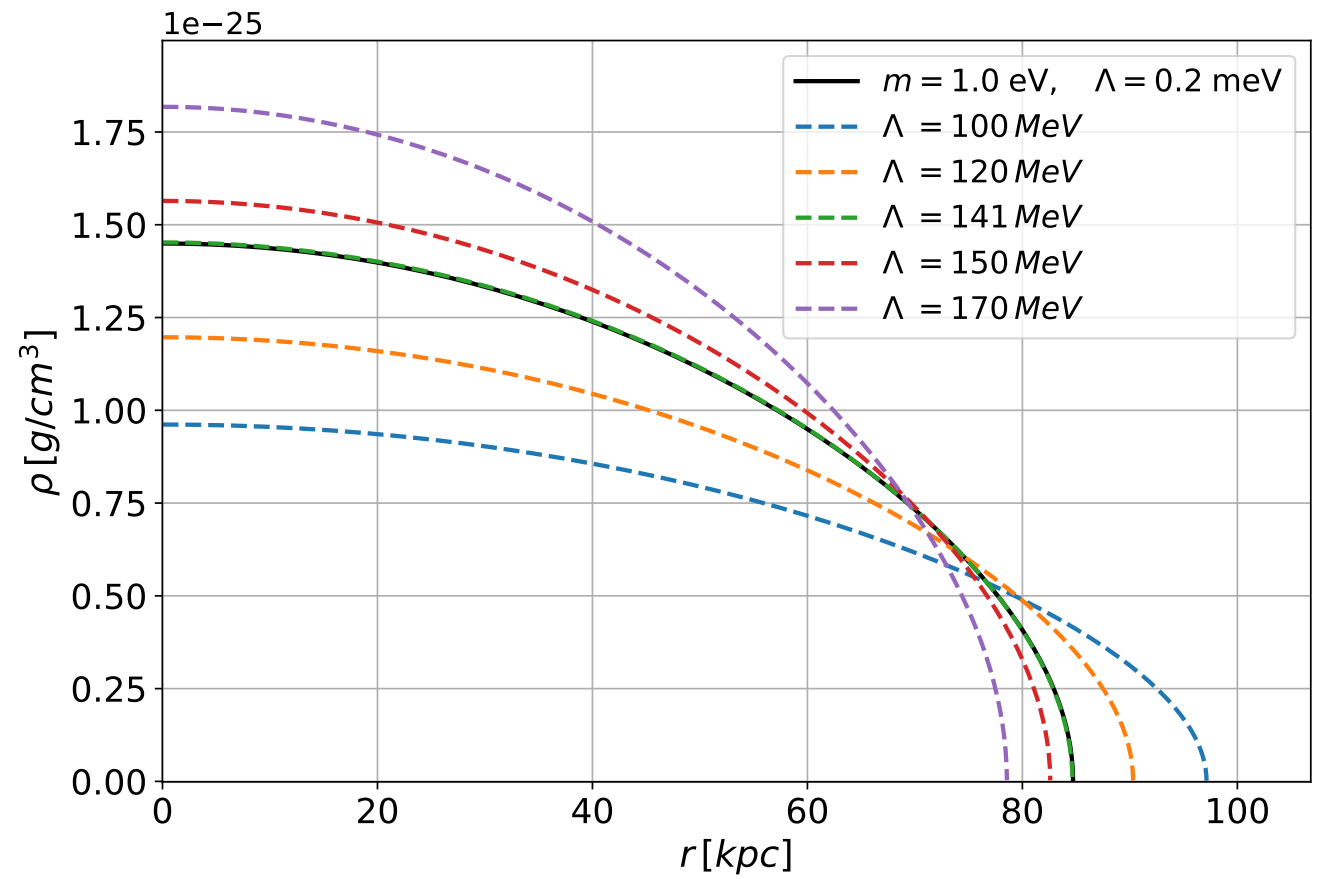

(a) $m_{\text {center }} \approx 1.1 \times 10^{-4} \mathrm{eV}$

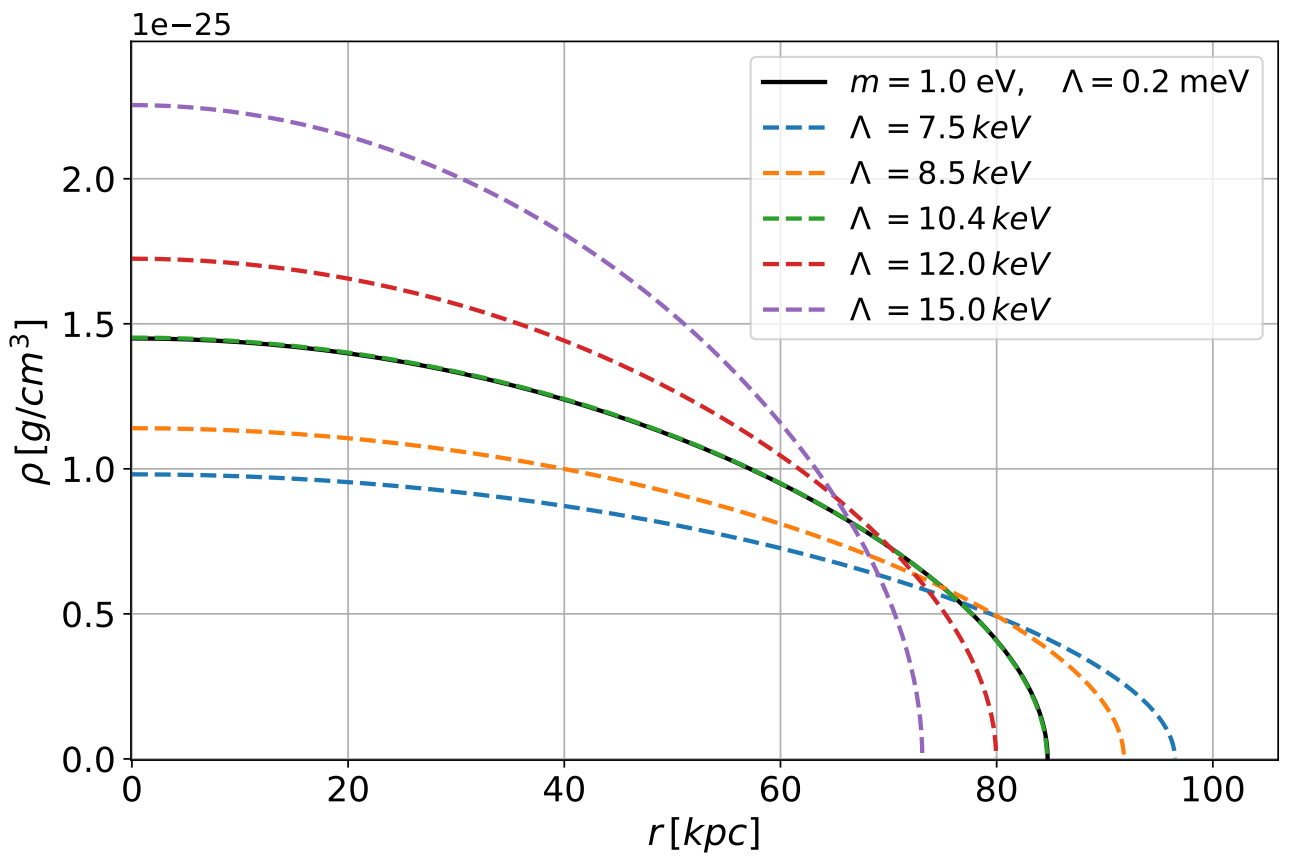

(b) $m_{\text {edge }} \approx 2.7 \times 10^{-3} \mathrm{eV}$

Figure 6.3: Dark matter density profile fitting for the values $m_{\text {center }} \approx 1.1 \times 10^{-4} \mathrm{eV}$ and $m_{\text {edge }} \approx 2.7 \times 10^{-3} \mathrm{eV}$. A larger value of $\Lambda$ is required, in both cases, for the halo profile to replicate that found in section 6.1. It is also evident that the smaller mass requires a larger $\Lambda$-value to correct for the changes. 


\section{The Self-Interaction}

As we now have new values for both $m$ and $\Lambda$, we may move on to checkpoint three. We have been able to reproduce the original density profile, meaning that we have the same value of the condensate radius as before, namely $84.7 \mathrm{kpc}$. In section 5.2 , we presented an expression for the thermalization radius of the dark matter - the radius at which the dark matter is able to thermalize and condense into a superfluid,

$$
R_{T} \lesssim 310 \mathrm{kpc}\left(\frac{\mathrm{m}}{\mathrm{eV}}\right)^{-8 / 7}\left(\frac{\mathrm{M}}{10^{12} \mathrm{M}_{\odot}}\right)^{1 / 7}\left(\frac{\sigma / \mathrm{m}}{\mathrm{cm}^{2} / \mathrm{g}}\right)^{2 / 7} .
$$

This expression depends upon the self-interaction cross section as shown in figure 6.4, for the $m_{\text {center }}$ case (the $m_{\text {edge }}$ case has the same shape, but with values five to six orders of magnitude larger on the x-axis).

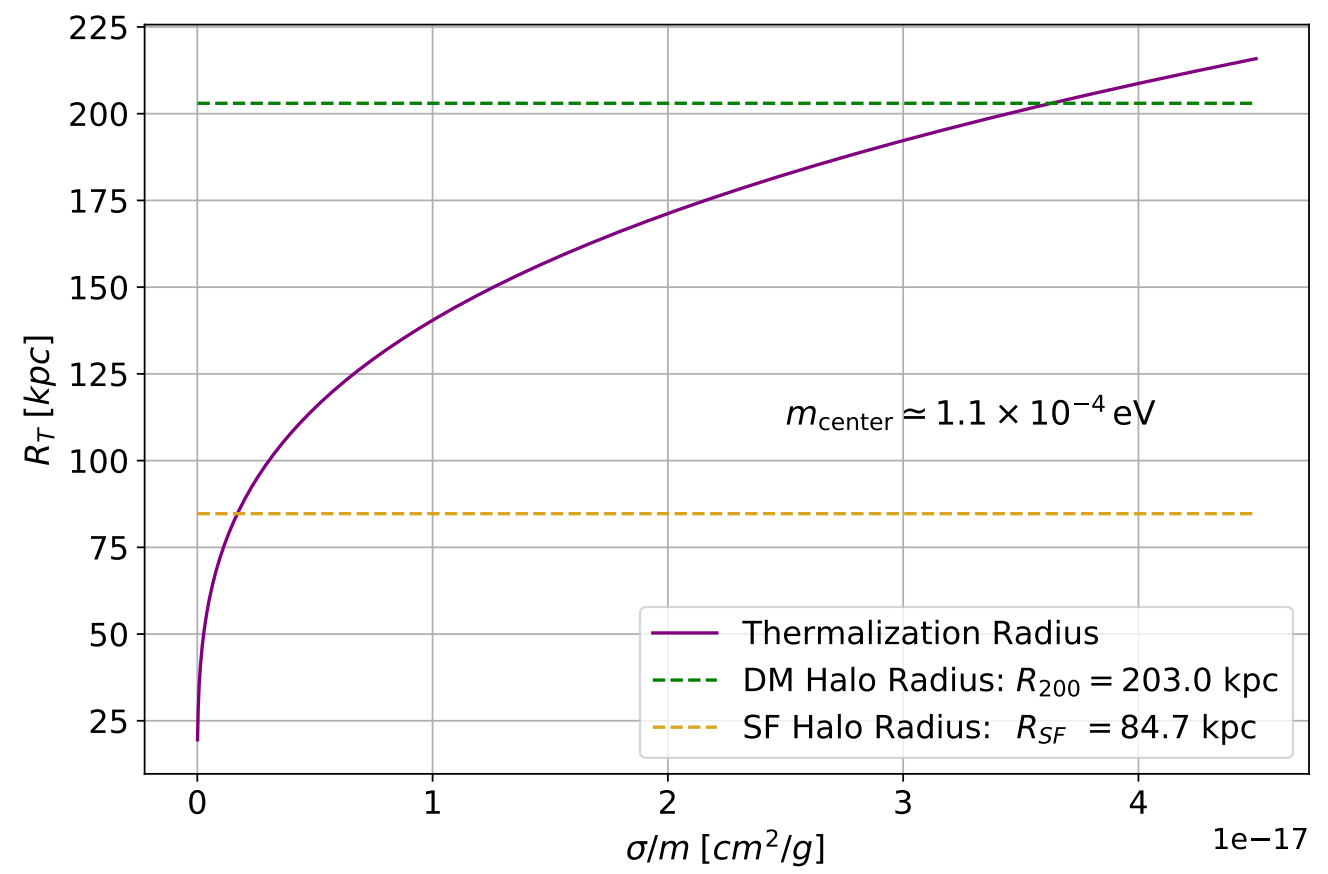

Figure 6.4: Cross section dependence of thermalization radius. The new $m_{\text {center }}$ and

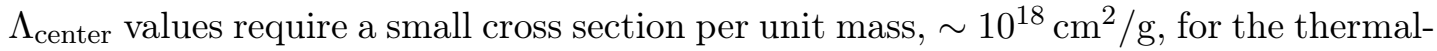
ization radius to be $84.7 \mathrm{kpc}$. As an additional comment, the superfluid (SF) halo radius displayed in this plot represents the upper limit of equation (6.30).

In the plot we have added a green and yellow dashed line, representing the virial radius and condensate radius, respectively. It is important that the thermalization, meaning the formation of the superfluid, happens within the DM halo of the galaxy. This is because the superfluid properties, and by that the MONDian behavior (see 
section 4.2), only should be viable at galactic scales. This requires us to limit the superfluid component to within the DM halo. Previously, we have used two different ways of defining the size of the condensate component, namely the thermalization radius (equation 6.30), and the radius where the zero temperature condensate density profile hits zero. In our case, we will assume the condensate radius and thermalization radius to be the same, which means that we now know which value the cross section must take. The value of the cross section for $R_{T}=84.7 \mathrm{kpc}$ is found by checking where the yellow dashed line intersects the purple one, which is approximately at

$$
\sigma / m_{\text {center }} \approx 1.7 \times 10^{-18} \mathrm{~cm}^{2} / \mathrm{g} \text { and } \sigma / \mathrm{m}_{\text {edge }} \approx 5.5 \times 10^{-13} \mathrm{~cm}^{2} / \mathrm{g}
$$

in the two different cases. We now have the answer to the first question of checkpoint three: decreasing $m$ and increasing $\Lambda$ results in a very low cross section for the dark matter particles.

Moving on to the second question means that we have to investigate any constraints on the cross section. The model presented in [1] requires galaxies to have a significant superfluid component in order to reproduce the observations that seem to favor a MONDian behavior. Galaxy clusters, on the contrary, should not have a large superfluid component, as we want them to behave more closely to the predictions of the $\Lambda$ CDM model. This property of the superfluid DM model is imposed in section VIII of [11], and it results in a lower bound on the DM particle mass. In combination with previous constraints (equation 6.27) this amounts to

$$
2.7 \mathrm{eV} \lesssim \mathrm{m}\left(\frac{\sigma / \mathrm{m}}{\mathrm{cm}^{2} / \mathrm{g}}\right)^{-1 / 4} \lesssim 4.2 \mathrm{eV},
$$

which may also be seen as a condition for the cross section, if the mass is already known. For our one meter vortex core center mass, this relation is plotted in figure 6.5 (again, the $m_{\text {edge }}$ case has the same shape, but with an x-axis five to six orders of magnitude higher).

As illustrated in figure 6.5, there is a small window for the value of the cross section which works together with our one meter vortex core mass. The maximum and minimum possible values of $\sigma / m$ are given as

$$
\begin{aligned}
\max : & \sigma / \mathrm{m}_{\text {center }} \approx 3.00 \times 10^{-18} \mathrm{~cm}^{2} / \mathrm{g} \\
\min : & \sigma / \mathrm{m}_{\text {center }} \approx 5.14 \times 10^{-19} \mathrm{~cm}^{2} / \mathrm{g}
\end{aligned}
$$

and

$$
\begin{aligned}
& \max : \quad \sigma / \mathrm{m}_{\text {edge }} \approx 9.72 \times 10^{-13} \mathrm{~cm}^{2} / \mathrm{g} \\
& \min : \sigma / \mathrm{m}_{\text {edge }} \approx 1.66 \times 10^{-13} \mathrm{~cm}^{2} / \mathrm{g} .
\end{aligned}
$$

As we can see, the value of $\sigma / m$ for a thermalization radius of $84.7 \mathrm{kpc}$, as presented in equation (6.31), lies within these boundaries in both cases. Based on this, the value of $\sigma / m$ is greatly affected by changes in $m$ and $\Lambda$, but all constraints of the model still seem to hold. 


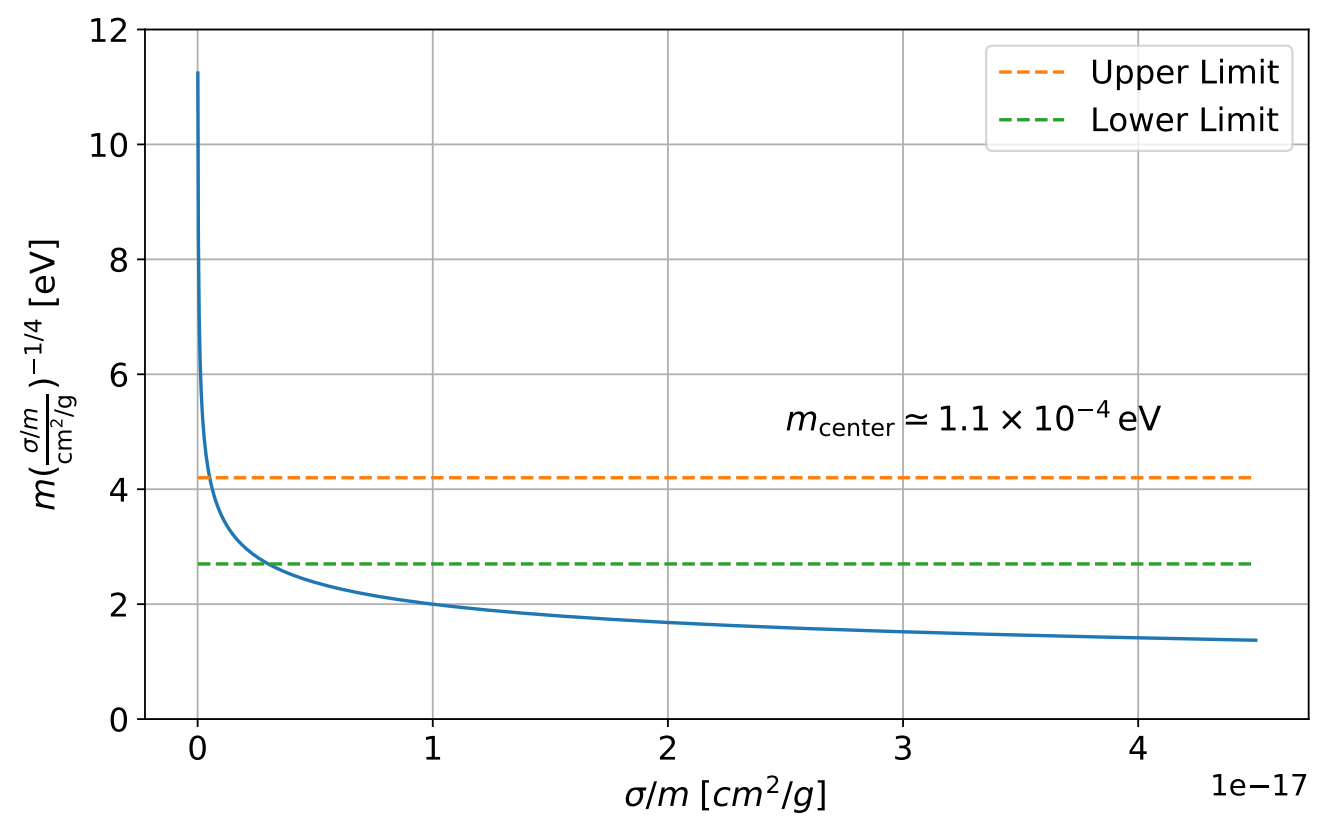

Figure 6.5: Visualization of the cross section constraints presented in equation (6.32), using a dark matter particle mass of $m_{\text {center }} \simeq 1.1 \times 10^{-4} \mathrm{eV}$. There is only a narrow window in which $\sigma / m$ fulfills the constraints for the given DM particle mass.

As a final remark, we should note that in imposing the constraint in equation (6.32), we have also revisited the initial constraint as mentioned at the end of the first checkpoint: The Particle Mass.

\section{The Temperature}

We have now found some values for which the vortices in the center and edge of the superfluid DM have a core size of $\sim 1 \mathrm{~m}$, the halo density profile looks as expected, and the model constraints seem to be fulfilled. The next step is then checkpoint four: is the halo temperature still below the critical temperature for the new parameter values? From section 5.3 we have an expression for the critical temperature:

$$
T_{c} \approx 4.5\left(\frac{\bar{f}}{100 \mathrm{~Hz}}\right) N^{1 / 3} \mathrm{nK} .
$$

However, this was for a halo of constant density. Still, for the critical temperature assessment, we need to use the full halo radius and not only the condensate component. We will therefore again, for simplicity, assume the density to be constant and equal to $\rho_{200}$. From this, we may take the same approach as in section 5.3. To do so, we must calculate $\bar{f}$ and $N$, where $\bar{f}$ is given as

$$
\bar{f}=\bar{\omega} / 2 \pi, \quad \bar{\omega}=\left(\omega_{x} \omega_{y} \omega_{z}\right)^{1 / 3},
$$


and $N$ is the total number of DM particles. Calculating the number of particles as $N=\rho_{200} V_{\text {halo }} / m$ and $\bar{f}$ from $\omega=\sqrt{4 \pi G \rho_{200} / 3}$, we get the critical temperature given in table 6.1 , for the two different particle masses. Here we have assumed the volume, $V_{\text {halo }}$, to be spherical and used the full DM halo radius of $R_{\text {halo }}=203 \mathrm{kpc}$. This should be fine, as the new values of $m$ and $\Lambda$ are chosen so that they fit the original halo profile. Further, we calculate the thermal energy, and thus temperature, of the dark matter by using the virial theorem on the following form:

$$
\frac{3}{5} \frac{G M}{R}=\frac{3}{2} \frac{k_{B} T}{m} .
$$

Here $M$ is the mass of the halo, $R$ the full radius and $m$ the DM particle mass, as usual. For the two different particle masses, we obtain the temperatures given in table 6.1 .

Table 6.1: Critical temperature and halo temperature when using DM particle masses as presented in equation (6.26).

\begin{tabular}{ccc}
\hline \hline DM particle mass & $T_{c}$ & $T_{D M}$ \\
\hline$m_{\text {center }} \simeq 1.1 \times 10^{-4} \mathrm{eV}$ & $0.34 \mathrm{~K}$ & $0.12 \mu \mathrm{K}$ \\
$m_{\text {edge }} \simeq 2.7 \times 10^{-3} \mathrm{eV}$ & $0.12 \mathrm{~K}$ & $2.93 \mu \mathrm{K}$ \\
\hline
\end{tabular}

As before, we obtain a dark matter temperature (way) below the critical temperature, consistent with the formation of a BEC.

\section{The Critical Angular Velocity}

In section 5.2 we calculated that the angular velocity of a Milky Way-like galaxy is of the order $\sim 10^{-16} \mathrm{~s}^{-1}$. We also calculated the critical angular velocity for vortex formation as

$$
\Omega_{c}=\frac{5}{2} \frac{\hbar^{2}}{m R^{2}} \ln \left(0.671 \frac{R}{\xi_{0}}\right),
$$

where $m$ is the DM particle mass, $R$ is the condensate radius and $\xi_{0}$ is the coherence length, or equivalently the vortex core size at the center of the condensate. This expression is based on a constant dark matter density, and for the length scales of a single vortex, we may assume this to be the case. However, this expression addresses the value of the critical angular velocity, which the whole condensate must be subject to, in order to have vortex formation. Still, within the condensate component, the density is not that far from constant (see figure 6.1), and we may use the expression in equation (6.40) to obtain an approximation of the magnitude of the critical angular velocity. 
For equation (6.40), we need the DM particle mass and the coherence length at the center of the condensate. For our particle mass that results in one meter vortex cores at the center of the condensate, this is straight forward. For the mass that results in a one meter vortex core at the edge, however, we need to calculate what the coherence length would be at the center. This is given by equation (5.15) and results in $\xi_{0} \sim 4 \mathrm{~cm}$. Inserting the two different masses, along with the coherence length at the center in each case, we get

$$
\begin{aligned}
\Omega_{c}^{\text {center }} & \sim 10^{-36} \mathrm{~s}^{-1}, \\
\Omega_{c}^{\text {edge }} & \sim 10^{-37} \mathrm{~s}^{-1} .
\end{aligned}
$$

This illustrates that the galaxy still rotates fast enough for vortex formation.

\section{The Energy of a Single Vortex}

Now, it is finally time for checkpoint six. The motivation behind making the vortex cores bigger was that it required a smaller mass, which in turn should increase the energy per vortex, as calculated at the end of section 5.2. The energy expression, given as

$$
E=\frac{4 \pi n(0,0)}{3} \frac{\hbar^{2}}{m} Z \ln \left(0.671 \frac{R}{\xi_{0}}\right),
$$

is based on a single vortex at the center and requires the number density and coherence length at the center. Again, this expression originates from the harmonic oscillator consideration, which means that it is valid for a constant density DM halo. Still, as we have argued before, around a single vortex core the density may be assumed constant, as the length scales are way smaller than the length scales over which the condensate density changes. In addition, this expression is based on a cylindrical condensate shape and not a spherical one. For a central vortex, we may therefore imagine, as a simple approach, taking a cylindrical sample around the vortex core and from that calculating the energy of the vortex. From this, we may insert $Z=2 R, n(0,0)=\rho(0) / m$, each of the particle masses and the individual central vortex core sizes. We then get

$$
\begin{aligned}
E_{\text {center }} & \approx 4.2 \times 10^{13} \mathrm{~J}, \\
E_{\text {edge }} & \approx 8.1 \times 10^{10} \mathrm{~J} .
\end{aligned}
$$

Converted to masses this becomes

$$
\begin{aligned}
& m_{\text {vortex }}^{\text {center }} \approx 4.8 \times 10^{-4} \mathrm{~kg}, \\
& m_{\text {vortex }}^{\text {edge }} \approx 9.0 \times 10^{-7} \mathrm{~kg} .
\end{aligned}
$$

From this we see that the smaller the particle DM mass, the larger the vortices and the more "mass" each vortex has. We still have $\sim 10^{26}$ vortices in the condensate, and adding all the masses together, in the center case, we obtain a total mass of $\sim 10^{22} \mathrm{~kg}$. This is of the same order of magnitude as Europa [66], one of the moons of Jupiter. 
We now know that decreasing the particle DM mass, and by that increasing the vortex core size, leads to a greater "mass" of each vortex. This again makes it more probable that the vortices could impact their surroundings. However, if all the vortices, in total, only amounts to the mass of a Jovian satellite, any huge impact seems improbable. Still, decreasing the particle mass even further would result in an increased energy per vortex. As long as we correct the mass change by increasing $\Lambda$, it seems like the model allows such parameter alterations. We will therefore test out a few smaller masses in section 6.5.

Altogether, it seems like a lower DM particle mass fulfills all the constraints of [11], and might be a possibility beyond the $\sim \mathrm{eV}$ mass scales presented in [1, 11]. Still, we have two checkpoints to go, namely number seven, The $\alpha$ parameter, and number eight, Rotation Curves. These will be assessed in the two following sections.

\subsection{The $\alpha$ Parameter}

In the superfluid DM model presented by Berezhiani and Khoury [1], it is the superfluid phonons that hold the key to the MONDian behavior, in the sense that they are governed by the MOND action (see beginning of section 3 in [1]). The MONDian force, essential for assuring the correct behavior of the model at galaxy scales, is ensured by the way the phonons couple to the baryons. The interaction term is given in their equation (26) as

$$
\mathscr{L}_{\text {int }}=-\alpha \frac{\Lambda}{M_{\mathrm{Pl}}} \theta \rho_{b},
$$

where $\rho_{b}$ is the baryon mass density, $\theta$ is a scalar field ${ }^{5}, M_{\mathrm{Pl}}$ is the Planck mass, $\Lambda$ is an energy scale originating from the MOND scalar action, and finally, $\alpha$ is a dimensionless parameter.

In addition to the two previous parameters, $m$ and $\Lambda$, to which we have fitted density profiles for various values in section 6.2 , there is an extra parameter of the model, the coupling constant $\alpha$. The parameters, $\Lambda$ and $\alpha$, may depend on temperature and will therefore take different values cosmologically than on galactic scales (see section 7 in [1]). The temperature dependence is, however, mild and may be ignored on galactic scales, which is our area of interest. In section 4 of the same paper, the superfluid phonons, and their relation to the baryons, are studied further, which results in a relation between the $\alpha$ and $\Lambda$ parameters, necessary to reproduce the MOND critical acceleration, $a_{0} \simeq 1.2 \times 10^{-8} \mathrm{~cm} / \mathrm{s}^{2}$ (equation (2) in [1]). This value differs only slightly from the one estimated by Milgrom in 1983 [6], presented in equation (4.2).

\footnotetext{
${ }^{5}$ In the superfluid interpretation, $\theta$ is the phase of a wave function, and it is therefore mentioned, together with the introduction of this interaction term, that from the superfluid perspective, this term may arise from the baryonic matter coupling to the superfluid vortices. This is quite exciting, as the vortices are our main point of interest. This statement was, however, hard to confirm from the associated sources listed in [1].
} 
The relation is given as follows:

$$
\alpha^{3 / 2} \Lambda=\sqrt{a_{0} M_{\mathrm{Pl}}} \simeq 0.8 \mathrm{meV},
$$

resulting in

$$
\alpha \simeq 0.86\left(\frac{\Lambda}{\mathrm{meV}}\right)^{-2 / 3} .
$$

For our original parameter, $\Lambda=0.2 \mathrm{meV}$, this results in the value

$$
\alpha \approx 2.51 \text {. }
$$

From the relation in equation (6.50), it is clear that the smaller masses explored in section 6.2 will lead to very small values of $\alpha$, as $\Lambda$ must increase in value in order to compensate for the new values of $m$. Based on equation (6.48), this is not something that invalidates our parameter variations, as it is the combination of $\alpha$ and $\Lambda$ that is of importance in the interaction term.

\subsection{Rotation Curves}

As we saw in section 2.2, an important factor leading the scientific community towards the idea of dark matter was the observation of flat galaxy rotation curves. Reproducing this is therefore important for the new superfluid DM model, and a large portion of [11] is therefore devoted to showing that the model indeed is able to produce realistic rotation curves. This was done for the parameter values $m=1 \mathrm{eV}$ and $\Lambda=0.05 \mathrm{meV}$, for two specific galaxies, where the results were compared to data and shown to provide a good fit. Based on the parameter variations that were performed in the previous section, it would be interesting to investigate whether or not some of the new parameter combinations are able to reproduce the results. To do so, we should follow the outline presented in [11], where a full dark matter density profile is calculated and also fitted to a Navarro-Frenk-White (NFW) profile outside the superfluid region. This, however, requires the numerical solution of several coupled differential equations, and as our main goal is not to reproduce the rotation curves perfectly, but rather figure out whether our new combinations of $m$ and $\Lambda$ will provide similar results as our original values, we may simplify the problem.

Instead of solving for the dark matter density profile and fitting it to an NFW profile at the outskirts, we will choose a simplified spherical density profile (a toy profile), along with using a toy spherical density profile for the baryons. These density profiles are needed in order to calculate the acceleration of a particle inside the galaxy and from that finding the circular velocity. In [11], equation (41), a toy profile for baryons was presented:

$$
\rho_{b}^{\text {toy }}(r)=\frac{M_{b}}{8 \pi L^{3}} e^{-r / L},
$$


where $L=2 \mathrm{kpc}$ is a radial length scale defining how far out in the galaxy we want most of the mass to be distributed. We will adopt this profile for the baryons, and also for the dark matter,

$$
\rho_{D M}^{t o y}(r)=\frac{M_{D M}}{8 \pi L_{D M}^{3}} e^{-r / L_{D M}},
$$

although with a different length scale, $L_{D M}=60 \mathrm{kpc}$. This is chosen to make sure that the dark matter is distributed far enough out to produce a flat rotation curve. The profiles are illustrated in figure 6.6.

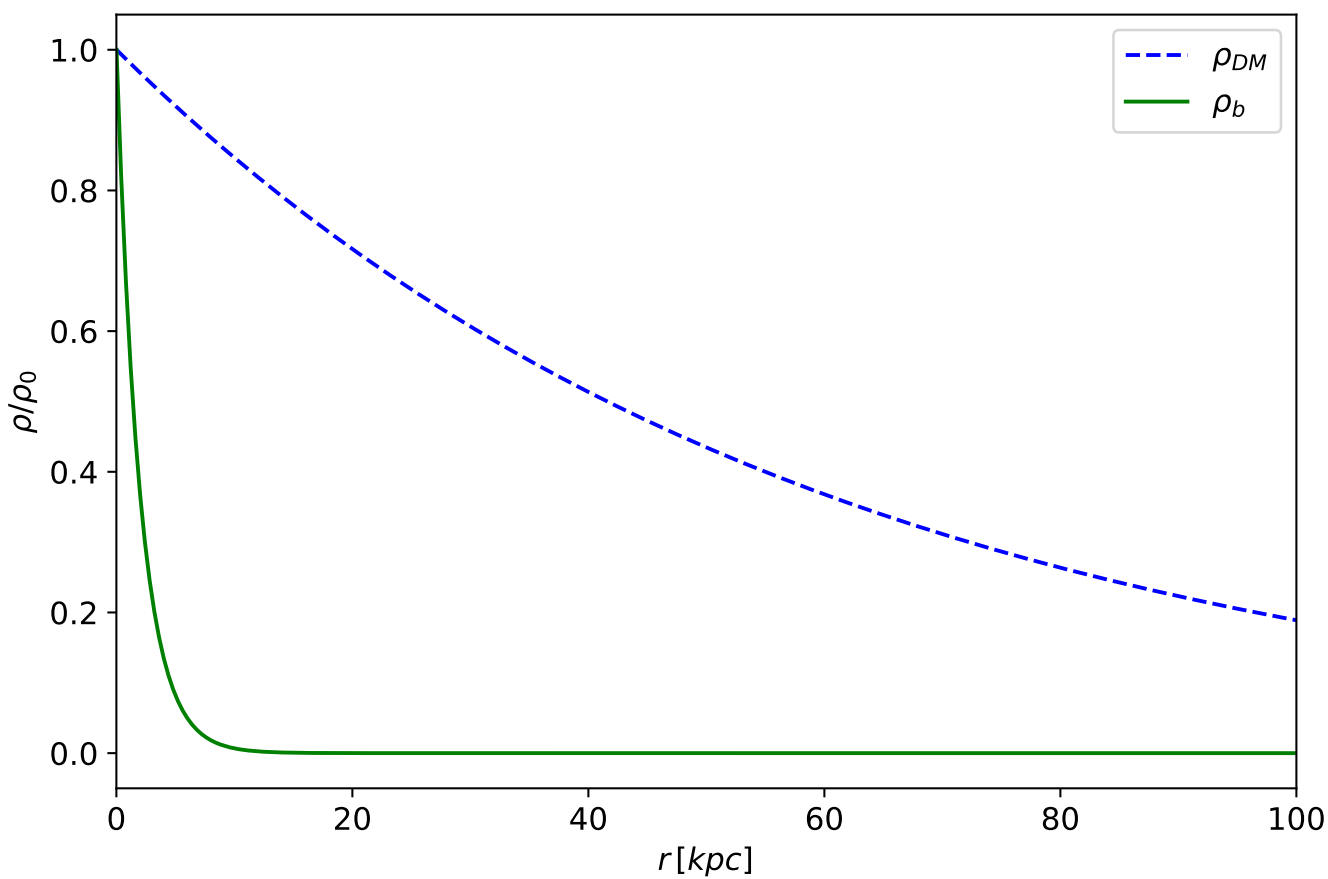

Figure 6.6: Toy density profiles that will be used to produce mock rotation curves. Both the dark matter and the baryons follow the same distribution, only with a different spatial extent. The curves are normalized.

We now want to calculate the radial acceleration on a test (baryonic) particle inside the galaxy. This is given by

$$
a=a_{b}(r)+a_{D M}(r)+a_{\text {phonon }}(r),
$$


where

$$
\begin{aligned}
a_{b}(r) & =G M_{b}(r) / r^{2} \\
a_{D M}(r) & =G M_{D M}(r) / r^{2} \\
a_{\text {phonon }}(r) & =\alpha \frac{\Lambda}{M_{\mathrm{Pl}}} \frac{d \phi}{d r},
\end{aligned}
$$

and $\phi$ is the phonon scalar field, governed by equation (32) in [11]:

$$
\frac{(\vec{\nabla} \phi)^{2}+2 m\left(\frac{2 \beta}{3}-1\right) \hat{\mu}}{\sqrt{(\vec{\nabla} \phi)^{2}+2 m(\beta-1) \hat{\mu}}} \vec{\nabla} \phi=\alpha M_{\mathrm{Pl}} \vec{a}_{b} .
$$

Here $\beta$ is a dimensionless constant introduced to parametrise finite-temperature effects ${ }^{6}$, set to $\beta=2$ in the aforementioned paper. We also have that $\hat{\mu}=\mu-m \Phi$, where $\mu$ is the chemical potential and $\Phi$ is the gravitational potential. The chemical potential will be calculated based on equation (48) of [1]

$$
\mu=\frac{\rho_{\text {center }}^{2}}{8 \Lambda^{2} m^{5}}
$$

where $\rho_{\text {center }}$ is given by equation (6.19) as before. This is based on the zero temperature case, but as our density profiles already are estimates it does not matter much in this scenario. The gravitational potential will be calculated as

$$
\nabla^{2} \Phi=4 \pi G\left(\rho_{D M}+\rho_{b}\right),
$$

assuming rotational symmetry. Equation (6.56) may be rewritten as a cubic equation of $(\vec{\nabla} \phi)^{2}$,

$$
\begin{aligned}
0= & (\vec{\nabla} \phi)^{6}+4 m\left(\frac{2 \beta}{3}-1\right) \hat{\mu}(\vec{\nabla} \phi)^{4}+4 m^{2}\left(\frac{2 \beta}{3}-1\right)^{2} \hat{\mu}^{2}(\vec{\nabla} \phi)^{2} \\
& -\alpha^{2} M_{\mathrm{Pl}}^{2} \vec{a}_{b}^{2}(\vec{\nabla} \phi)^{2}-2 m(\beta-1) \hat{\mu} \alpha^{2} M_{\mathrm{Pl}}^{2} \vec{a}_{b}^{2},
\end{aligned}
$$

where roots may be found numerically. For $m$ and $\Lambda$-values that fulfill the constraints, only one root will be real.

To solve equation (6.54), we need the total mass of the galaxy along with an initial value of the gravitational potential at the center. The total baryonic mass is set to $M_{b}=10^{11} M_{\odot}$, and the total dark matter mass to $10 M_{b}$. The initial gravitational potential value is in [11] decided so that we obtain the total mass when integrating the density over the full volume of the galaxy. We choose the initial value in the same way.

\footnotetext{
${ }^{6}$ The condensate profile calculated in section 6.1 was for the zero temperature case, but in reality the dark matter will have some finite small temperature.
} 
To solve equation (6.54) we take a numerical approach, and below we present an overview of the procedure:

1. Choose values of $m$ and $\Lambda$.

2. Calculate constants $\left(\alpha, \rho_{\text {center }}\right.$ and $\left.\mu\right)$.

3. Choose initial position (somewhere close to the galactic center) and final position (somewhere outside halo).

4. Call function that solves for gravitational potential, $\Phi$ :

(a) Initialize $\Phi, M_{b}, M_{D M},(\vec{\nabla} \phi)^{2}, \rho_{S F}$ and $\rho_{b}$.

(b) Assume that $\Phi=\Phi(r)$ and, for each step outwards, calculate $\frac{d^{2} \Phi}{d r^{2}}=-\frac{2}{r} \frac{d \Phi}{d r}+$ $4 \pi G\left(\rho_{D M}+\rho_{b}\right)$.

(c) Calculate $\frac{d \Phi}{d r}$ and $\Phi$ using ODE-solver of choice.

(d) Find new current values of $M_{D M}, M_{b}, \rho_{S F}, \rho_{b}$ and $(\vec{\nabla} \phi)^{2}$ to be used in next step. These values are obtained by calling individual functions that give the density from the toy profiles, integrates the toy profiles to obtain the enclosed mass and finds the roots of equation (6.59). The latter requires $\vec{a}_{b}^{2}$, which is found from equation (6.55).

(e) Once this is done we check the total obtained mass, $\left(M_{D M}+M_{b}\right)$, to make sure that we end up with a total mass equal to the one we initially chose $\left(M_{b}=10^{11} M_{\odot}, M_{D M}=10 M_{b}\right)$.

(f) We then return $\Phi(r), \rho_{S F}(r), \rho_{b}(r), M_{D M}(r), M_{b}(r),(\vec{\nabla} \phi)^{2}$ and $r$.

5. Now we may calculate $a$, equation (6.54), by calling a function where $a_{b}, a_{D M}$ and $a_{\text {phonon }}$ are calculated as functions of $r$. Here we assume that $\sqrt{(\vec{\nabla} \phi)^{2}}=\frac{d \phi}{d r}$.

Once equation (6.54) has been solved, we may use the formula

$$
a=\frac{v_{\text {circ }}^{2}}{r},
$$

to obtain the corresponding rotation curve. Figure 6.7 shows the rotation curves for our original parameters, $m=1 \mathrm{eV}$ and $\Lambda=0.2 \mathrm{meV}$, along with the one meter center and edge parameters of section 6.2 , and one smaller mass that will be investigated in section 6.5. The rotation curve with the values $m=1 \mathrm{eV}$ and $\Lambda=0.05 \mathrm{meV}$ is also included.

Although the profiles used in this case do not represent the real distribution of dark matter and baryons in a galaxy, it seems like there is still an important result here. The variations in parameters (blue, green, red and yellow lines), done in the previous sections, result in the same rotation curves. This indicates that an alteration in the parameters of the model could be possible and does not remove the model's ability to reproduce realistic rotation curves. This should, however, also be checked for 


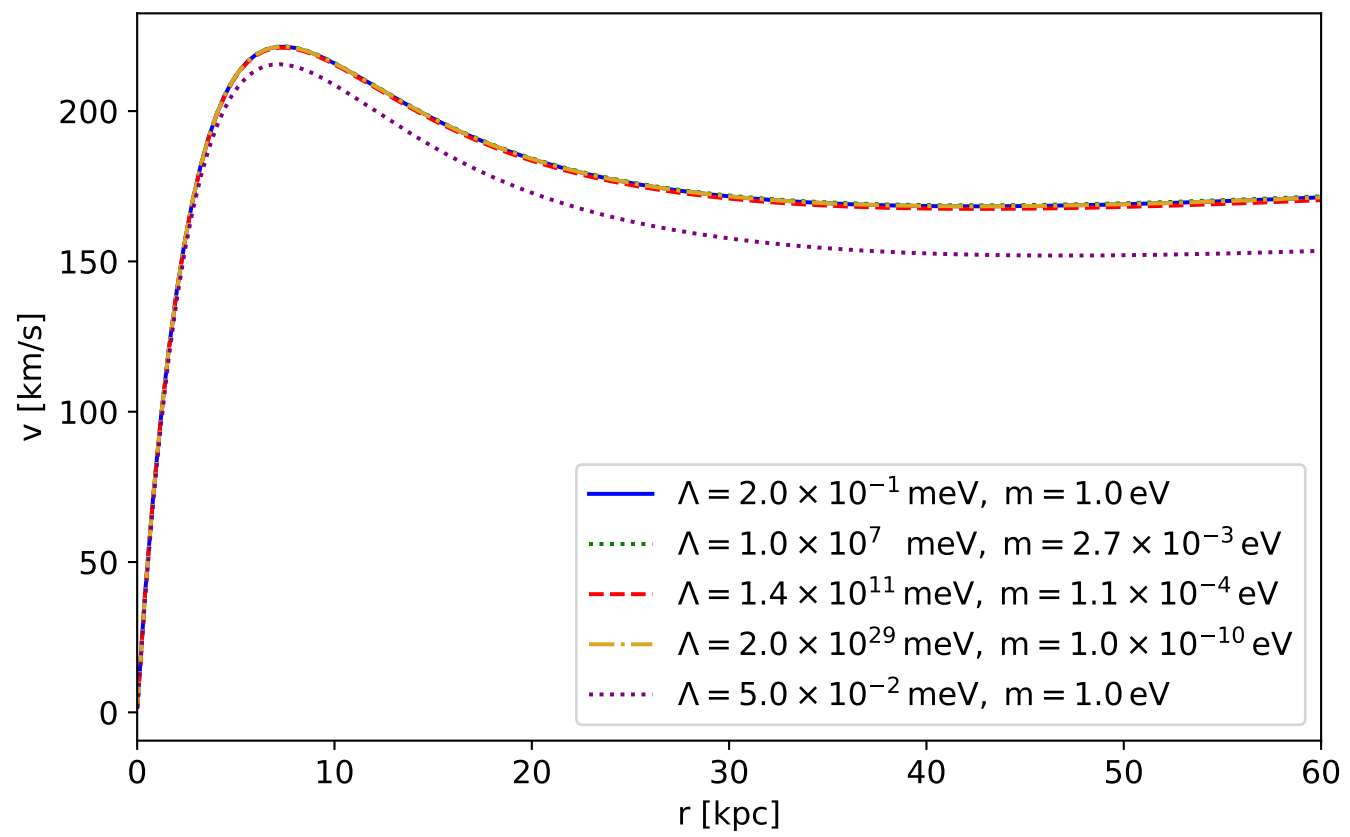

Figure 6.7: Resulting rotation curves of the toy density profiles presented in equation (6.52) and (6.53) for various combinations of $m$ and $\Lambda$. The blue, green, red and yellow lines result from our original $m$ and $\Lambda$ values, and the combinations obtained in section 6.2 and later in section 6.5. The purple line is a result of the parameters $m=1 \mathrm{eV}$ and $\Lambda=0.05 \mathrm{meV}$, which have not been decided based on the same process as the others, and do not fulfill equation (6.20).

a more accurate dark matter density profile and actual baryonic density distribution observations. The last curve (purple) is included to illustrate that we get a different rotation curve when we change $\Lambda$ without changing the mass (compared to the blue line).

We have now investigated the parameters of the superfluid dark matter model, which opens up for the possibility of different dark matter particle masses, and as a result, larger vortex cores. In particular, we have looked at a one meter vortex core scenario and found it plausible. Assuming all constraints to be in order, we would now like to test out a few even smaller masses, to check if there are any limits to the variations of the parameters. 


\subsection{DM Particle Mass Variation}

In section 6.2 we tested two values of $m$ that strayed beyond the $\sim \mathrm{eV}$ range proposed in [1]. In this section we will investigate a few more, namely $m=10^{-7} \mathrm{eV}, m=10^{-10} \mathrm{eV}$ and $m=10^{-15} \mathrm{eV}$. In [1], the DM candidate who enables the superfluidity is described as "axion-like" with a mass in the $\mathrm{eV}$ range. The mass of the axion has been proposed to be as low as $\sim 10^{-22} \mathrm{eV}$ in fuzzy dark matter models [67], but is more conventionally thought to be in the $\sim 10^{-5}-10^{-3} \mathrm{eV}$ range [68]. In addition, we found in section 6.2 that smaller particle masses, and by that larger vortex cores, result in a higher vortex energy. This again gives each vortex a higher "mass," which further increases their gravitational pull, making any interaction between the vortices and baryons more likely.

In figure 6.8 , we have gone through the same process of choosing a value of $m$ and finding a value of $\Lambda$ that fits the density profile, as was done in section 6.2. For each of the new mass values we also repeat the vortex core size calculation (equation 5.15) and the calculation giving the energy/mass of a single vortex at the center of the condensate (see end of section 5.2). An overview of the $\Lambda$-values required by the different masses is given in table 6.2, along with the vortex core size, energy and mass.

Table 6.2: Overview of model parameters and resulting vortex properties.

\begin{tabular}{ccccc}
\hline \hline DM particle mass & $\Lambda$-value & Vortex core & Vortex energy & Vortex "mass" \\
\hline$m=10^{-7} \mathrm{eV}$ & $\sim 2 \times 10^{8} \mathrm{GeV}$ & $1.1 \times 10^{3} \mathrm{~m}$ & $4.7 \times 10^{19} \mathrm{~J}$ & $5.2 \times 10^{2} \mathrm{~kg}$ \\
$m=10^{-10} \mathrm{eV}$ & $\sim 2 \times 10^{17} \mathrm{GeV}$ & $1.1 \times 10^{6} \mathrm{~m}$ & $4.7 \times 10^{25} \mathrm{~J}$ & $5.2 \times 10^{8} \mathrm{~kg}$ \\
$m=10^{-15} \mathrm{eV}$ & $\sim 2 \times 10^{32} \mathrm{GeV}$ & $1.1 \times 10^{11} \mathrm{~m}$ & $4.7 \times 10^{35} \mathrm{~J}$ & $5.2 \times 10^{18} \mathrm{~kg}$ \\
\hline
\end{tabular}

From table 6.2 we again observe that the lower the value of the mass, the higher the value of $\Lambda$ required to reproduce the density profile of figure 6.1 becomes. As we take a look at the required $\Lambda$-values, we find that this trend will result in a limit of the parameter variations. From the table we see that the resulting $\Lambda$-value for the $m=10^{-10} \mathrm{eV}$ case gets close to the reduced Planck mass of $M_{\mathrm{Pl}}=\sqrt{\hbar c / 8 \pi G} \approx 2.435 \times$ $10^{18} \mathrm{GeV}$. Just to put this number into perspective, the highest possible energy of the collisions performed by the Large Hadron collider (LHC) at CERN is $1.3 \times 10^{4} \mathrm{GeV}$ [69]. This is the most powerful accelerator in the world (at the given time), but the value of the Planck mass is still $10^{14}$ times greater than the highest energy levels available to it. In other words, already for a mass of $m=10^{-10} \mathrm{eV}$, we arrive at huge energy values of $\Lambda$, which is the parameter that decides the validity range of the model. For $m=10^{-15} \mathrm{eV}$, the $\Lambda$-value needed to fit the original density profile is immense, a massive $10^{14}$ times the Planck mass scales and $10^{28}$ times the maximum energy levels reached by the LHC. 
The Planck mass is in itself no upper or lower limit, only a mass carefully constructed by combinations of $\hbar, c$ and $G$, but reaching these energy levels is usually seen as a sign that the theory is missing something or may not be well understood without the use of quantum gravity. Based on this, there is therefore no need to go even further down to $m \sim 10^{-22} \mathrm{eV}$ (which from the inverse relation of the mass and $\Lambda$-values shown in figure 6.8 would be of the order of $10^{52}-10^{55} \mathrm{GeV}$.)

Table 6.2 also shows that we may obtain very large vortex cores and corresponding vortex "masses." However, for the smallest DM particle mass, we reach a vortex core size larger than the separation of the vortices in the condensate. This means that the vortices would overlap and that we would need a new description of the system. As a comparison, for type II superconductors ${ }^{7}$, the superconductive state would cease to exist when the vortex cores start overlapping. For a Bose-Einstein condensate, however, vortex lattices can remain stable even in these situations (see chapter 9 and section 9.5 of [39]). Still, combined with the fact that the $\Lambda$-value is way above the Planck mass scale, a DM particle mass as low as $10^{-15} \mathrm{eV}$ seems quite improbable in the current superfluid dark matter model. The $10^{-10} \mathrm{eV}$ particle mass is also on the border of Planck mass scales, and yet all $10^{26}$ vortices combined only amount to a mass of $\sim 10000 \mathrm{M}_{\odot}$. The Milky Way contains $\sim 10^{11}$ stars, and the gravitational impact of an extra 10000 Suns would most likely be negligible in the big picture. Adding all the vortices of the $m=10^{-7} \mathrm{eV}$ case together, we end up at a total mass of ten times the mass of Jupiter.

At this point, we must remember that the expressions used to calculate the vortex core size and energy were based on a constant halo density. Previously, we have continued using the formulas as the vortices have been so small that the density could be assumed constant in their proximity. At least for the lowest particle dark matter mass, we can no longer assume the density to be constant, and the numbers presented in table 6.2 must therefore be treated with caution.

From these simple tests, it is clear that it is possible to find a $\Lambda$-value that will reproduce the desired density profile for very small values of $m$. However, how small the particle mass can get is limited by the fact that $\Lambda$ increases significantly as the mass is decreased, quickly leading us beyond Planck energy scales. We have also seen that too low values of $m$ will lead to the vortex cores overlapping, which would change the description of the system, and possibly affect the phonons needed to mediate the MONDian force. The lowest particle DM mass still resulting in a $\Lambda$-value below the Planck mass scale only gives a total vortex mass of roughly $10000 \mathrm{M}_{\odot}$, which most likely will have little to no effect on the galaxy as a whole. Based on the results of this section, and the results of section 6.2 , it seems like there is a little wiggle room in the $m \sim \mathrm{eV}$ and $\Lambda \sim \mathrm{meV}$ scales originally presented in $[1,11]$. However, any vortex influence seems unlikely.

\footnotetext{
${ }^{7} \mathrm{~A}$ superconductor is a type of material that, when cooled down to a critical temperature, has no electrical resistance [70]. This is obtained by applying external magnetic fields to the superconductor. A type I superconductor only remains in the superconducting state for weak magnetic fields. A type II superconductor can withstand more powerful magnetic fields, and is characterized by the formation of magnetic vortices in the applied magnetic field [71].
} 
After the investigation and results of this chapter, it would be interesting to check if the baryons could have any impact on the vortices instead. This will be done in chapter 7, by introducing an additional term to the Lagrangian of section 4.3.

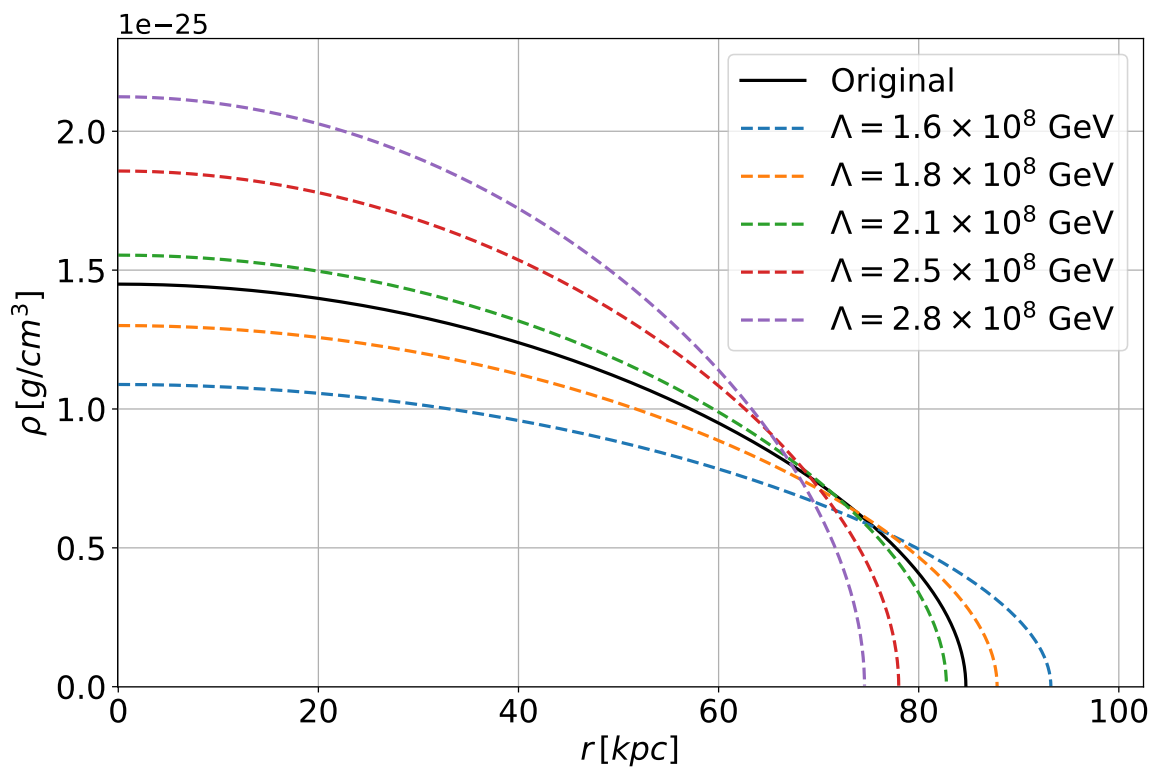

(a) $m=10^{-7} \mathrm{eV}$

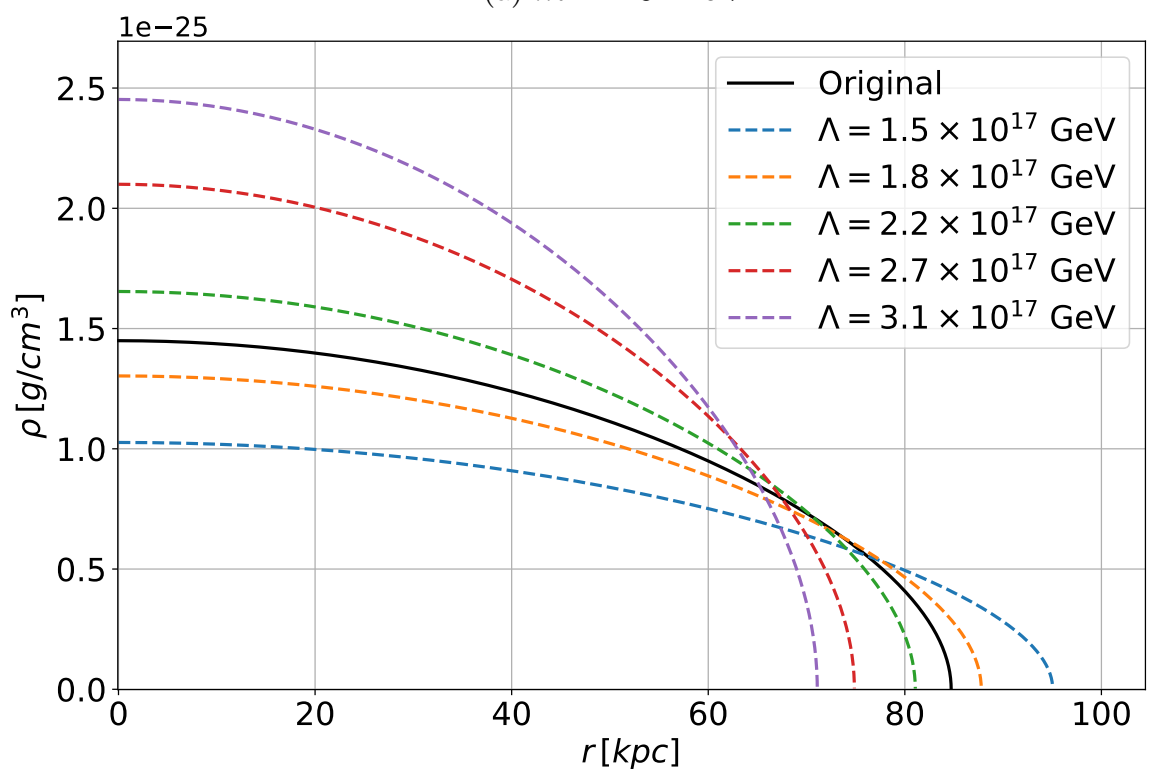

(b) $m=10^{-10} \mathrm{eV}$

Figure 6.8: Dark matter density profile fitting for various values of $m$. The lower the value of the DM particle, the higher the value of $\Lambda$ required to reproduce the original density profile. It is worth noting that the mass of $m=10^{-15} \mathrm{eV}$ requires a $\Lambda$-value well over the reduced Planck mass, $M_{\mathrm{Pl}} \approx 2.435 \times 10^{18} \mathrm{GeV}$. 


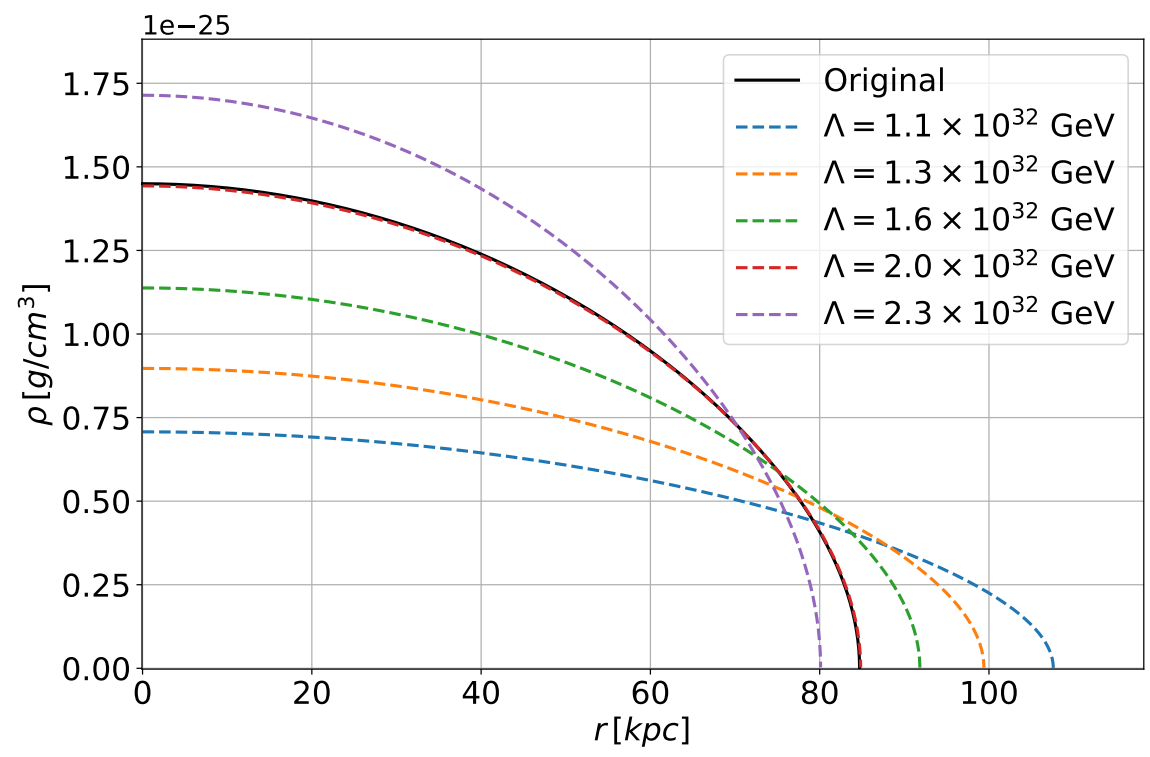

(c) $m=10^{-15} \mathrm{eV}$

Figure 6.8: - Continued. 


\section{Chapter 7}

\section{Vortex-Baryon Interaction}

"A friendship older than your civilization, and infinitely more complex."

- Missy, Doctor Who: The Magician's Apprentice.

In this chapter we want to investigate what will happen if we introduce baryons to the superfluid through an interaction term in the Lagrangian. As we have seen in chapter 5 , vortices will form in the superfluid DM, as it rotates way above the critical angular velocity. As calculated in chapter 6 , the vortices will be very small, and probably have little impact on their surroundings. Even when we create larger and more "massive" vortices by altering the mass and energy scales originally proposed for the model in $[1,11]$, it seems unlikely that the vortices will have any noticeable impact on their immediate surroundings. Based on this, we could ask the opposite question: will the baryons affect the vortices?

In section 7.1 we present a simple interaction term suitable as an extension to the Lagrangian of section 4.3 , and in section 7.2 we look at the resulting equations of motion.

\subsection{The Interaction Term}

As mentioned, we now want to study the possible interaction between the vortices forming in the superfluid and the baryonic matter in the galaxy. The simplest way to do this is to add an interaction term to the superfluid Lagrangian presented in equation (4.8). To fulfill Lorentz invariance in Minkowski spacetime (see section VI in [72]), we propose a current-current interaction term ${ }^{1}$ :

$$
\mathscr{L}_{I}=f j_{S F}^{\mu} j_{\mu b} .
$$

As far as we know, the expression presented in equation (7.1) is the only Lorentz invariant interaction term [72], and thus our only possible choice. Here $j_{S F}^{\mu}$ is the

\footnotetext{
${ }^{1}$ This form may also be seen in other settings, such as in electroweak theory (see e.g. section 20.2 in [57])
} 
conserved current obtained from the continuity equation found in section 4.3 (equation 4.21) and $j_{\mu b}$ is the corresponding conserved current for the baryons. The currents have the form

$$
j_{S F}^{\mu}=\rho_{S F}^{2} \partial^{\mu} \psi, \quad j_{b}^{\mu}=\rho_{b} v_{b}^{\mu},
$$

where the baryonic current is based on the definition of flux ${ }^{2}$. Adding the vortex-baryon interaction term (7.1) to the Lagrangian density of the superfluid (4.8) results in

$$
\mathscr{L}=\partial_{\mu} \phi^{*} \partial^{\mu} \phi-m^{2}|\phi|^{2}-\lambda|\phi|^{4}+f j_{S F}^{\mu} j_{\mu b},
$$

where $f$ is a constant. There has only been added one new term to the Lagrangian density, namely the interaction term. For now, we have neglected any kinetic energy term for the baryons, as there in general is a lot more dark matter than baryons. As we are studying the behavior of the dark matter and not the baryons, this should be a valid first approximation.

\subsection{The Equations of Motion}

We now want to find equations of motion that describe how a vortex is affected by the presence of baryons. To do so, we use the parametrization

$$
\phi(X)=\frac{\rho_{S F}(X)}{\sqrt{2}} e^{i \psi(X)},
$$

introduced in section 4.3. Inserting this into equation (7.3) yields

$$
\mathscr{L}=\frac{1}{2} \partial_{\mu} \rho_{S F} \partial^{\mu} \rho_{S F}+\frac{1}{2} \rho_{S F}^{2}\left(\partial_{\mu} \psi \partial^{\mu} \psi-m^{2}\right)-\frac{1}{4} \lambda \rho_{S F}^{4}+f j_{S F}^{\mu} j_{\mu b},
$$

where the last term can be written as

$$
f j_{S F}^{\mu} j_{\mu b}=f \rho_{S F}^{2} \partial^{\mu} \psi \rho_{b} v_{\mu b}
$$

using equation (7.2). Let us now derive the equations of motion with respect to the condensate parameters, $\rho_{S F}$ and $\psi$ :

$$
\frac{\partial \mathscr{L}}{\partial \rho_{S F}}-\partial_{\eta} \frac{\partial \mathscr{L}}{\partial\left(\partial_{\eta} \rho_{S F}\right)}=0
$$

and

$$
\frac{\partial \mathscr{L}}{\partial \psi}-\partial_{\eta} \frac{\partial \mathscr{L}}{\partial\left(\partial_{\eta} \psi\right)}=0
$$

\footnotetext{
${ }^{2}$ In the case of baryons, we have mass moving around in space, and $\rho_{b}$ is the related mass density. If we have a velocity field, $\boldsymbol{v}_{b}$, describing the movement of the baryons, a flux may be defined as $\boldsymbol{j}=\rho_{b} \boldsymbol{v}_{b}$.
} 
From equation (7.5) and (7.6) we get

$$
\begin{aligned}
\frac{\partial \mathscr{L}}{\partial \rho_{S F}} & =\rho_{S F}\left(\partial_{\mu} \psi \partial^{\mu} \psi-m^{2}\right)-\lambda \rho_{S F}^{3}+2 f \rho_{S F} \partial^{\mu} \psi \rho_{b} v_{\mu b}, \\
\frac{\partial \mathscr{L}}{\partial\left(\partial_{\eta} \rho_{S F}\right)} & =\frac{\partial}{\partial\left(\partial_{\eta} \rho_{S F}\right)}\left(\frac{1}{2} \partial_{\mu} \rho_{S F} g^{\mu \nu} \partial_{\nu} \rho_{S F}\right) \\
& =\frac{1}{2} g^{\mu \nu}\left(\partial_{\nu} \rho_{S F} \delta_{\mu}^{\eta}+\partial_{\mu} \rho_{S F} \delta_{\nu}^{\eta}\right) \\
& =\frac{1}{2} \partial^{\eta} \rho_{S F}+\frac{1}{2} \partial^{\eta} \rho_{S F} \\
& =\partial^{\eta} \rho_{S F}, \\
\frac{\partial \mathscr{L}}{\partial \psi} & =0, \\
\frac{\partial \mathscr{L}}{\partial\left(\partial_{\eta} \psi\right)} & =\frac{\partial}{\partial\left(\partial_{\eta} \psi\right)}\left(\frac{1}{2} \rho_{S F}^{2}\left[\partial_{\mu} \psi g^{\mu \nu} \partial_{\nu} \psi-m^{2}\right]+f \rho_{S F}^{2} g^{\mu \nu} \partial_{\nu} \psi \rho_{b} v_{\mu b}\right) \\
& =\frac{1}{2} \rho_{S F}^{2}\left(g^{\mu \nu} \partial_{\nu} \psi \delta_{\mu}^{\eta}+g^{\mu \nu} \partial_{\mu} \psi \delta_{\nu}^{\eta}\right)+f \rho_{S F}^{2} g^{\mu \nu} \delta_{\nu}^{\eta} \rho_{b} v_{\mu b} \\
& =\frac{1}{2} \rho_{S F}^{2}\left(\partial^{\eta} \psi+\partial^{\eta} \psi\right)+f \rho_{S F}^{2} \rho_{b} v_{b}^{\eta} \\
& =\rho_{S F}^{2} \partial^{\eta} \psi+f \rho_{S F}^{2} \rho_{b} v_{b}^{\eta},
\end{aligned}
$$

and equation (7.7) and (7.8) become

$$
\partial_{\eta} \partial^{\eta} \rho_{S F}=\rho_{S F}\left(\partial_{\mu} \psi \partial^{\mu} \psi-m^{2}-\lambda \rho_{S F}^{2}+2 f \partial^{\mu} \psi \rho_{b} v_{\mu b}\right)
$$

and

$$
\partial_{\eta}\left(\rho_{S F}^{2} \partial^{\eta} \psi+f \rho_{S F}^{2} \rho_{b} v_{b}^{\eta}\right)=0
$$

These equations are very similar to equations (4.20) and (4.21) obtained in section 4.3, but have an additional term due to the vortex-baryon interaction.

Let us first rewrite equation (7.13). Again we use the assumptions (see section 4.3)

$$
\begin{aligned}
\psi & =n \theta, \quad n \in \mathbb{N}, \\
\rho_{S F} & =\rho_{S F}(r), \\
\rho_{b} & =\rho_{b}(r, \theta), \\
v_{b} & =v_{b}(r, \theta), \\
\partial_{0} \psi & =\mu,
\end{aligned}
$$


in cylindrical coordinates. Then we also have

$$
\begin{aligned}
\nabla \psi & =\frac{\partial \psi}{\partial r} \vec{e}_{r}+\frac{1}{r} \frac{\partial \psi}{\partial \theta} \vec{e}_{\theta}+\frac{\partial \psi}{\partial z} \vec{e}_{z} \\
\nabla^{2} \rho & =\frac{1}{r} \frac{\partial}{\partial r}\left(r \frac{\partial \rho}{\partial r}\right)+\frac{1}{r^{2}} \frac{\partial^{2} \rho}{\partial \theta^{2}}+\frac{\partial^{2} \rho}{\partial z^{2}}
\end{aligned}
$$

To rewrite the equation on a simpler form, we again follow section 3.2 in [55] and introduce the variables

$$
R=\frac{\rho_{S F}}{\rho_{0}}, \quad \rho_{0}^{2}=\frac{\mu^{2}-m^{2}}{\lambda}, \quad \eta=\sqrt{\lambda} \rho_{0} r
$$

This leads to

$$
\begin{aligned}
& \partial_{\eta} \partial^{\eta} \rho_{S F}=\rho_{S F}\left(\partial_{\mu} \psi \partial^{\mu} \psi-m^{2}-\lambda \rho_{S F}^{2}+2 f \partial^{\mu} \psi \rho_{b} v_{\mu b}\right) \\
&-\nabla^{2} \rho_{S F}=\rho_{S F}\left[\mu^{2}-(\nabla \psi)^{2}-m^{2}-\lambda \rho_{S F}^{2}+2 f \rho_{b}\left(\mu v_{0 b}-\nabla \psi \cdot \boldsymbol{v}_{b}\right)\right] \\
&-\frac{1}{r} \frac{\partial}{\partial r}\left(r \frac{\partial \rho_{S F}}{\partial r}\right)=\rho_{S F}\left[\rho_{0}^{2} \lambda-\lambda \rho_{S F}^{2}-\left(\frac{n}{r}\right)^{2}+2 f \rho_{b}\left(\mu v_{0 b}-v_{\theta b} \frac{n}{r}\right)\right] \\
&-\frac{1}{r} \frac{\partial}{\partial r}\left(r \frac{\partial \rho_{S F}}{\partial r}\right)=\rho_{S F}\left[\rho_{0}^{2} \lambda\left(1-\frac{\rho_{S F}^{2}}{\rho_{0}^{2}}\right)-\frac{n^{2}}{r^{2}}+2 f \rho_{b}\left(\mu v_{0 b}-v_{\theta b} \frac{n}{r}\right)\right] \\
&-\frac{\sqrt{\lambda} \rho_{0}}{\eta} \frac{\partial \eta}{\partial r} \frac{\partial}{\partial \eta}\left[\frac{\eta}{\sqrt{\lambda} \rho_{0}} \frac{\partial \eta}{\partial r} \frac{\partial}{\partial \eta}\left(R \rho_{0}\right)\right]=R \rho_{0}\left[\frac{\eta^{2}}{r^{2}}\left(1-R^{2}\right)-\frac{n^{2}}{\eta^{2}} \lambda \rho_{0}^{2}\right. \\
&\left.+2 f \rho_{b}\left(\mu v_{0 b}-v_{\theta b} \frac{n}{\eta} \sqrt{\lambda} \rho_{0}\right)\right] \\
&-\frac{1}{\eta} \frac{\partial}{\partial \eta}\left[\eta \frac{\partial R}{\partial \eta}\right]=R\left[1-R^{2}-\frac{n^{2}}{\eta^{2}}+\frac{2 f \rho_{b}}{\lambda \rho_{0}^{2}}\left(\mu v_{0 b}-v_{\theta b} \frac{n}{\eta} \sqrt{\lambda} \rho_{0}\right)\right] \\
& \frac{1}{\eta} \frac{\partial}{\partial \eta}\left[\eta \frac{\partial R}{\partial \eta}\right]+\left(1-\frac{n^{2}}{\eta^{2}}\right) R-R^{3}=2 f \rho_{b} R\left(\frac{n}{\eta} \frac{v_{\theta b}}{\sqrt{\lambda} \rho_{0}}-\frac{\mu v_{0 b}}{\lambda \rho_{0}^{2}}\right)
\end{aligned}
$$

where we have used $g_{\mu \nu}=\operatorname{diag}(1,-1,-1,-1)$ as stated in [55]. The resulting vortex equation is similar to equation (4.36), only now with a source term on the right-hand side. If the baryons have no effect on the vortex, the right-hand side would be equal to zero. Firstly, remember that in special relativity, the four-velocity is given as

$$
v^{\mu}=\frac{d x^{\mu}}{d \tau}=\gamma \frac{d x^{\mu}}{d t}=\gamma(c, \boldsymbol{v})
$$

where $\gamma={\sqrt{1-v^{2} / c^{2}}}^{-1} \approx 1$ for non-relativistic velocities. As we are using natural units, we therefore have $v_{0 b}=c=1$. For the right-hand side of equation (7.25) to be 
zero (for all values of $R$ ) we therefore need

$$
\begin{aligned}
0 & =2 f \rho_{b} R\left(\frac{n}{\eta} \frac{v_{\theta b}}{\sqrt{\lambda} \rho_{0}}-\frac{\mu v_{0 b}}{\lambda \rho_{0}^{2}}\right) \\
0 & =\frac{n}{\eta} \frac{v_{\theta b}}{\sqrt{\lambda} \rho_{0}}-\frac{\mu}{\lambda \rho_{0}^{2}} \\
v_{\theta b} & =\frac{\mu}{\lambda \rho_{0}^{2}} \frac{\eta \sqrt{\lambda} \rho_{0}}{n} \\
v_{\theta b} & =\frac{\mu}{n} r .
\end{aligned}
$$

In other words, if the baryons were to have no impact on the vortex, their velocity in the $\theta$-direction should increase with the radius out from the vortex core, and would also depend upon the phase of the superfluid as $\mu=\partial_{0} \psi$. For large radii, this would result in extremely large velocities, which is unrealistic. This would, of course, look different for Lagrangian densities made for the superfluid DM case, but as of now it seems unlikely that surrounding baryons do not affect the vortex. The $\rho_{S F}$-profile will therefore, in the case where vortex-baryon interaction is included in equation (4.8), look different from the profile illustrated in figure 4.3. Without the exact form of $v_{\theta b}$, we can not solve equation (7.25). To find this expression, we would have to make up a new Lagrangian density which incorporates the bosonic fluid along with the baryons. This means that we would have to add a kinetic energy term for the baryons and also take gravity into account. This will have to be assessed in future works, and possibly for Lagrangians more similar to the one presented in section 6 of [1]. Going forth to study equation (7.14), we therefore lack an expression for $\rho_{S F}$.

In the case of equation (4.8) (Lagrangian with no interaction term), the resulting Euler-Lagrange equation with respect to $\psi$ (equation 4.21) was trivial when applying the assumptions of equation (7.15). Now, we have an additional term as a result of the vortex-baryon interaction and equation (7.14),

$$
\partial_{\eta}\left(\rho_{S F}^{2} \partial^{\eta} \psi+f \rho_{S F}^{2} \rho_{b} v_{b}^{\eta}\right)=0,
$$

is not trivially fulfilled anymore. As we have not obtained an expression for $\rho_{S F}$ from equation (7.25), we will not be able to solve equation (7.31). However, it could be interesting to take a look at it, to see if we can gain some further insight:

$$
\begin{aligned}
& 0=\partial_{\eta}\left(\rho_{S F}^{2} \partial^{\eta} \psi+f \rho_{S F}^{2} \rho_{b} v_{b}^{\eta}\right) \\
& 0=2 \rho_{S F}\left(\partial_{\eta} \rho_{S F}\right) \partial^{\eta} \psi+\rho_{S F}^{2} \partial_{\eta} \partial^{\eta} \psi+2 f \rho_{S F}\left(\partial_{\eta} \rho_{S F}\right) \rho_{b} v_{b}^{\eta} \\
& +f \rho_{S F}^{2}\left(\partial_{\eta} \rho_{b}\right) v_{b}^{\eta}+f \rho_{S F}^{2} \rho_{b}\left(\partial_{\eta} v_{b}^{\eta}\right) \\
& 0=2 \rho_{S F}\left(\partial_{0} \rho_{S F}\right) \partial_{0} \psi-2 \rho_{S F} \boldsymbol{\nabla} \rho_{S F} \cdot \nabla \psi+\rho_{S F}^{2}\left(\partial_{0}\left(\partial_{0} \psi\right)-\nabla^{2} \psi\right) \\
& +2 f \rho_{S F} \rho_{b}\left(\partial_{0} \rho_{S F}\right) v_{0 b}-2 f \rho_{S F} \rho_{b} \boldsymbol{\nabla} \rho_{S F} \cdot \boldsymbol{v}_{b}+f \rho_{S F}^{2}\left(\partial_{0} \rho_{b}\right) v_{0 b} \\
& -f \rho_{S F}^{2} \boldsymbol{v}_{b} \cdot \nabla \rho_{b}+f \rho_{S F}^{2} \rho_{b}\left(\partial_{0} v_{0 b}-\nabla \cdot \boldsymbol{v}_{b}\right)
\end{aligned}
$$




$$
\begin{gathered}
0=-2 \rho_{S F} \boldsymbol{\nabla} \rho_{S F} \cdot \boldsymbol{\nabla} \psi-2 f \rho_{b} \rho_{S F} \boldsymbol{v}_{b} \cdot \nabla \rho_{S F}-f \rho_{S F}^{2} \boldsymbol{v}_{b} \cdot \nabla \rho_{b} \\
+f \rho_{S F}^{2} \rho_{b}\left(\partial_{0} v_{0 b}-\nabla \cdot \boldsymbol{v}_{b}\right) \\
0=-2 f \rho_{b} \rho_{S F} v_{r b} \frac{d \rho_{S F}}{d r}-f \rho_{S F}^{2}\left(v_{r b} \frac{\partial \rho_{b}}{\partial r}+\frac{v_{\theta b}}{r} \frac{\partial \rho_{b}}{\partial \theta}\right) \\
-f \rho_{S F}^{2} \rho_{b}\left(\frac{1}{r} \frac{\partial\left(r v_{r b}\right)}{\partial r}+\frac{1}{r} \frac{\partial v_{\theta b}}{\partial \theta}\right) .
\end{gathered}
$$

To solve equation (7.36) we need further information about the different variables $\rho_{b}$, $\rho_{S F}, v_{r b}$ and $v_{\theta b}$. As of now, we have two equations (7.25 and 7.36) and four different variables. To obtain the information we need, we must either make some assumptions regarding the behavior of a few of the variables, or set up a more complex Lagrangian density with a higher quantity of dynamical variables. We did try to add a kinetic energy term for the baryons, to check if we could use some of the baryonic variables to calculate additional Euler-Lagrange equations of the system. This, however, did not result in any new information, and will be further discussed in chapter 9 .

If we had discovered previously that the baryons did not affect the vortex, we could have assumed the approximate vortex solution for our vortex profile, $R=\eta / \sqrt{2+\eta^{2}}$. If we in addition assume that $v_{\theta b}=v_{\theta b}(r)$, and that the baryonic density is approximately constant close to a vortex, we can get an equation that describes the radial baryonic velocity. This gives a radial velocity that goes towards zero for large radii, while increasing exponentially towards the center of the vortex. This would imply that the baryons were moving fast inwards or outwards from the vortex core, but standing still further out, meaning that there would have to be some accumulation of particles around the vortex. This would again break with the assumption that the baryonic density is constant on vortex scales, showing that the assumptions and results are inconsistent. What we need is therefore some additional information to solve our system. This must most likely come in the form of a Lagrangian density which incorporates all details of the system.

Based on all of this, there is a lot of work left for future investigations. However, this simple assessment did tell us that the baryons seem to affect the vortex in some way or another. This means that we should expect the vortex profile of the superfluid DM model to deviate somewhat from that of a single straight-line vortex, as illustrated in figure 4.3.

Now that we have investigated the possible impact of the vortices on the baryons (chapter 6), and vice versa, the possible impact of the baryons on the vortices (this chapter), it is time to move on to the vortices themselves. In chapter 8 we will study the Lagrangian density that describes the new superfluid dark matter model and see if we can find a vortex solution similar to that of section 4.3.1. 


\section{Chapter 8}

\section{Superfluid Dark Matter Lagrangian}

"Space, the final frontier. Final, because it wants to kill us."

- The Doctor, Doctor Who: Oxygen.

So far we have only worked with a somewhat simple superfluid Lagrangian, inspired by the works in [55], and in section 4.3.1 we found a known vortex solution of the EulerLagrange equations. In [1], Berezhiani and Khoury present a full relativistic Lagrangian that describes the superfluid dark matter model:

$$
\mathscr{L}=-\frac{1}{2}\left(\left|\partial_{\mu} \phi\right|^{2}+m^{2}|\phi|^{2}\right)-\frac{\Lambda^{4}}{6\left(\Lambda_{c}^{2}+|\phi|^{2}\right)^{6}}\left(\left|\partial_{\mu} \phi\right|^{2}+m^{2}|\phi|^{2}\right)^{3} .
$$

Here $\Lambda$ is a constant of $\sim \mathrm{eV}$ scale, originating from the MOND Lagrangian, and $\Lambda_{c}$ is introduced to allow a vacuum solution $(\phi=0)$. Equation (8.1) is a relativistic completion of equation (6.1), in the zero temperature limit. We now want to work with this Lagrangian density to see if it also has a vortex solution, as we know from condensed matter physics that vortices should form in a rotating superfluid.

\subsection{The Klein-Gordon Equation}

When ignoring the complicated last term of equation (8.1), it should reduce to the regular Klein-Gordon equation,

$$
-\partial_{0}^{2} \phi+\nabla^{2} \phi=m^{2} \phi
$$

the relativistic energy-momentum relation on quantized form. Ensuring that this is the case will help us know which metric signature to use, either $(-,+,+,+)$ or $(+,-,-,-)$, as this is not specified in [1]. First, let us rewrite the version of equation (8.1) without 
the last term,

$$
\begin{aligned}
\mathscr{L} & =-\frac{1}{2}\left(\left|\partial_{\mu} \phi\right|^{2}+m^{2}|\phi|^{2}\right) \\
& =-\frac{1}{2} g^{\mu \nu} \partial_{\mu} \phi \partial_{\nu} \phi^{*}-\frac{1}{2} m^{2} \phi \phi^{*}
\end{aligned}
$$

We then find the Euler-Lagrange equations with respect to $\phi^{*}$ :

$$
\frac{\partial \mathscr{L}}{\partial \phi^{*}}-\partial_{\eta} \frac{\partial \mathscr{L}}{\partial\left(\partial_{\eta} \phi^{*}\right)}=0
$$

The two terms are

$$
\begin{gathered}
\frac{\partial \mathscr{L}}{\partial \phi^{*}}=-\frac{1}{2} m^{2} \phi \\
\text { and } \\
\partial_{\eta} \frac{\partial \mathscr{L}}{\partial\left(\partial_{\eta} \phi^{*}\right)}=-\frac{1}{2}\left[\left(\partial_{\eta} g^{\mu \eta}\right) \partial_{\mu} \phi-g^{\mu \eta} \partial_{\eta}\left(\partial_{\mu} \phi\right)\right] .
\end{gathered}
$$

Assuming Minkowski space, $g_{\mu \nu}= \pm \operatorname{diag}(-1,1,1,1)$, the Euler-Lagrange equations become

$$
\begin{aligned}
-\frac{1}{2} m^{2} \phi+\frac{1}{2}\left[\left(\partial_{\eta} g^{\mu \eta}\right) \partial_{\mu} \phi+g^{\mu \eta} \partial_{\eta}\left(\partial_{\mu} \phi\right)\right] & =0 \\
-m^{2} \phi+g^{\mu \eta} \partial_{\eta}\left(\partial_{\mu} \phi\right) & =0 \\
-m^{2} \phi+g^{00} \partial_{0}^{2} \phi+g^{i i} \partial_{i}^{2} \phi & =0
\end{aligned}
$$

where $i$ runs over the spatial coordinates. To reproduce equation (8.2) we must choose the metric $g_{\mu \nu}=\operatorname{diag}(-1,1,1,1)$, and we must therefore use the metric signature $(-,+,+,+)$ in the next section, when trying to find a vortex solution of equation (8.1).

\subsection{Equations of Motion}

We now want to check if we can extract an equation of motion describing the vortices in the model presented by Berezhiani and Khoury. For detailed calculations concerning every step, please visit appendix A. Let us again state the Lagrangian density:

$$
\mathscr{L}=-\frac{1}{2}\left(\left|\partial_{\mu} \phi\right|^{2}+m^{2}|\phi|^{2}\right)-\frac{\Lambda^{4}}{6\left(\Lambda_{c}^{2}+|\phi|^{2}\right)^{6}}\left(\left|\partial_{\mu} \phi\right|^{2}+m^{2}|\phi|^{2}\right)^{3} .
$$

As before, we will use the expression

$$
\phi=\frac{\rho(X)}{\sqrt{2}} e^{i \psi(X)}
$$


for the wavefunction, assume a static solution so that $\partial_{0} \rho=0$, and use $\partial_{0} \psi=\mu$. We have omitted the label $S F$, as it is clear in this context that it is the superfluid properties that are being calculated. This gives the term

$$
\left|\partial_{\mu} \phi\right|^{2}=\frac{1}{2} g^{\mu \nu} \partial_{\mu} \rho \partial_{\nu} \rho+\frac{1}{2} g^{\mu \nu} \rho^{2} \partial_{\mu} \psi \partial_{\nu} \psi
$$

to be inserted into equation (8.11), and the Lagrangian now has the form

$$
\begin{aligned}
\mathscr{L}= & -\frac{1}{4}\left(g^{\mu \nu} \partial_{\mu} \rho \partial_{\nu} \rho+g^{\mu \nu} \rho^{2} \partial_{\mu} \psi \partial_{\nu} \psi+m^{2} \rho^{2}\right) \\
& -\frac{\Lambda^{4}}{48\left(\Lambda_{c}^{2}+\frac{1}{2} \rho^{2}\right)^{6}}\left(g^{\mu \nu} \partial_{\mu} \rho \partial_{\nu} \rho+g^{\mu \nu} \rho^{2} \partial_{\mu} \psi \partial_{\nu} \psi+m^{2} \rho^{2}\right)^{3} .
\end{aligned}
$$

To properly keep track of all the indices, we write out the quadratic term as follows:

$$
\begin{aligned}
\mathscr{L}=-\frac{1}{4}\left(g^{\mu \nu} \partial_{\mu} \rho \partial_{\nu} \rho\right. & \left.+g^{\mu \nu} \rho^{2} \partial_{\mu} \psi \partial_{\nu} \psi+m^{2} \rho^{2}\right) \\
-\frac{\Lambda^{4}}{48\left(\Lambda_{c}^{2}+\frac{1}{2} \rho^{2}\right)^{6}} & \left(g^{\mu \nu} \partial_{\mu} \rho \partial_{\nu} \rho+g^{\mu \nu} \rho^{2} \partial_{\mu} \psi \partial_{\nu} \psi+m^{2} \rho^{2}\right) \\
\times & \left(g^{\alpha \beta} \partial_{\alpha} \rho \partial_{\beta} \rho+g^{\alpha \beta} \rho^{2} \partial_{\alpha} \psi \partial_{\beta} \psi+m^{2} \rho^{2}\right) \\
\times & \left(g^{\omega \sigma} \partial_{\omega} \rho \partial_{\sigma} \rho+g^{\omega \sigma} \rho^{2} \partial_{\omega} \psi \partial_{\sigma} \psi+m^{2} \rho^{2}\right) .
\end{aligned}
$$

In section 4.3.1, we obtained a vortex equation by finding the Euler-Lagrange equation with respect to $\rho$,

$$
\frac{\partial \mathscr{L}}{\partial \rho}-\partial_{\eta} \frac{\partial \mathscr{L}}{\partial\left(\partial_{\eta} \rho\right)}=0
$$

and we will again take the same approach here. Let us start with the easiest part $^{1}$, and look at equation (8.15) to find

$$
\begin{aligned}
\frac{\partial \mathscr{L}}{\partial \rho}= & -\frac{1}{2}\left(g^{\mu \nu} \rho \partial_{\mu} \psi \partial_{\nu} \psi+m^{2} \rho\right) \\
& +\frac{\Lambda^{4} \rho}{8\left(\Lambda_{c}^{2}+\frac{1}{2} \rho^{2}\right)^{7}}\left(g^{\mu \nu} \partial_{\mu} \rho \partial_{\nu} \rho+g^{\mu \nu} \rho^{2} \partial_{\mu} \psi \partial_{\nu} \psi+m^{2} \rho^{2}\right)^{3} \\
& -\frac{\Lambda^{4}}{8\left(\Lambda_{c}^{2}+\frac{1}{2} \rho^{2}\right)^{6}}\left(g^{\mu \nu} \rho \partial_{\mu} \psi \partial_{\nu} \psi+m^{2} \rho\right)\left(g^{\alpha \beta} \partial_{\alpha} \rho \partial_{\beta} \rho+g^{\alpha \beta} \rho^{2} \partial_{\alpha} \psi \partial_{\beta} \psi+m^{2} \rho^{2}\right)^{2}
\end{aligned}
$$

Further, we use equation (8.15) to find

$$
\begin{aligned}
\frac{\partial \mathscr{L}}{\partial\left(\partial_{\eta} \rho\right)}=-\frac{1}{2} g^{\eta \nu} \partial_{\nu} \rho & -\frac{\Lambda^{4}}{8\left(\Lambda_{c}^{2}+\frac{1}{2} \rho^{2}\right)^{6}} g^{\eta \nu} \partial_{\nu} \rho \\
& \times\left(g^{\alpha \beta} \partial_{\alpha} \rho \partial_{\beta} \rho+g^{\alpha \beta} \rho^{2} \partial_{\alpha} \psi \partial_{\beta} \psi+m^{2} \rho^{2}\right) \\
& \times\left(g^{\omega \sigma} \partial_{\omega} \rho \partial_{\sigma} \rho+g^{\omega \sigma} \rho^{2} \partial_{\omega} \psi \partial_{\sigma} \psi+m^{2} \rho^{2}\right) .
\end{aligned}
$$

\footnotetext{
${ }^{1}$ Clearly not implicating that this is easy, but rather referring to the lesser of two evils.
} 
This again gives:

$$
\begin{aligned}
\partial_{\eta} \frac{\partial \mathscr{L}}{\partial\left(\partial_{\eta} \rho\right)}= & -\frac{1}{2}\left[\left(\partial_{\eta} g^{\eta \nu}\right) \partial_{\nu} \rho+g^{\eta \nu} \partial_{\eta}\left(\partial_{\nu} \rho\right)\right] \\
& +\frac{6 \Lambda^{4} \rho\left(\partial_{\eta} \rho\right)}{8\left(\Lambda_{c}^{2}+\frac{1}{2} \rho^{2}\right)^{7}} g^{\eta \nu} \partial_{\nu} \rho\left(g^{\alpha \beta} \partial_{\alpha} \rho \partial_{\beta} \rho+g^{\alpha \beta} \rho^{2} \partial_{\alpha} \psi \partial_{\beta} \psi+m^{2} \rho^{2}\right)^{2} \\
& -\frac{\Lambda^{4}}{8\left(\Lambda_{c}^{2}+\frac{1}{2} \rho^{2}\right)^{6}}\left[\left(\partial_{\eta} g^{\eta \nu}\right) \partial_{\nu} \rho+g^{\eta \nu} \partial_{\eta}\left(\partial_{\nu} \rho\right)\right] \\
& \times\left(g^{\alpha \beta} \partial_{\alpha} \rho \partial_{\beta} \rho+g^{\alpha \beta} \rho^{2} \partial_{\alpha} \psi \partial_{\beta} \psi+m^{2} \rho^{2}\right)^{2} \\
& -\frac{\Lambda^{4}}{4\left(\Lambda_{c}^{2}+\frac{1}{2} \rho^{2}\right)^{6}} g^{\eta \nu} \partial_{\nu} \rho\left[\left(\partial_{\eta} g^{\alpha \beta}\right) \partial_{\alpha} \rho \partial_{\beta} \rho+g^{\alpha \beta} \partial_{\eta}\left(\partial_{\alpha} \rho\right) \partial_{\beta} \rho+g^{\alpha \beta} \partial_{\alpha} \rho \partial_{\eta}\left(\partial_{\beta} \rho\right)\right. \\
& +\left(\partial_{\eta} g^{\alpha \beta}\right) \rho^{2} \partial_{\alpha} \psi \partial_{\beta} \psi+g^{\alpha \beta} \partial_{\eta}\left(\rho^{2}\right) \partial_{\alpha} \psi \partial_{\beta} \psi+g^{\alpha \beta} \rho^{2} \partial_{\eta}\left(\partial_{\alpha} \psi\right) \partial_{\beta} \psi \\
& \left.+g^{\alpha \beta} \rho^{2} \partial_{\alpha} \psi \partial_{\eta}\left(\partial_{\beta} \psi\right)+m^{2} \partial_{\eta}\left(\rho^{2}\right)\right] \\
& \times\left(g^{\omega \sigma} \partial_{\omega} \rho \partial_{\sigma} \rho+g^{\omega \sigma} \rho^{2} \partial_{\omega} \psi \partial_{\sigma} \psi+m^{2} \rho^{2}\right) .
\end{aligned}
$$

We want to check if we can extract a vortex solution from the Euler-Lagrange equations. To do so, we will work in polar coordinates and also insert what led to our previous vortex equation, mainly that $\rho_{S F}=\rho_{S F}(r), \psi=n \theta$ and $\partial_{0} \psi=\mu$ (see section 4.3.1). In addition, we know that our metric is diagonal, $g_{\mu \nu}=\operatorname{diag}\left(-1,1, \mathrm{r}^{2}, 1\right)$, and as we are using polar coordinates, the indices cycle through $\mu=t, r, \theta, z$ (where $c=1$ ). We will also use the notation " " to represent the derivative with respect to $r$. Inserting all of this into equation (8.17) results in

$$
\frac{\partial \mathscr{L}}{\partial \rho}=-\frac{1}{2} q \rho+\frac{\Lambda^{4} \rho}{8\left(\Lambda_{c}^{2}+\frac{1}{2} \rho^{2}\right)^{7}}\left(\dot{\rho}^{2}+q \rho^{2}\right)^{3}-\frac{\Lambda^{4}}{8\left(\Lambda_{c}^{2}+\frac{1}{2} \rho^{2}\right)^{6}} q \rho\left(\dot{\rho}^{2}+q \rho^{2}\right)^{2},
$$

if we define

$$
q=m^{2}-\mu^{2}+\frac{n^{2}}{r^{2}}
$$

For equation (8.19) we obtain

$$
\begin{aligned}
\partial_{\eta} \frac{\partial \mathscr{L}}{\partial\left(\partial_{\eta} \rho\right)}= & -\frac{1}{2} \ddot{\rho}+\frac{3 \Lambda^{4} \rho \dot{\rho}^{2}}{4\left(\Lambda_{c}^{2}+\frac{1}{2} \rho^{2}\right)^{7}}\left(\dot{\rho}^{2}+q \rho^{2}\right)^{2}-\frac{\Lambda^{4}}{8\left(\Lambda_{c}^{2}+\frac{1}{2} \rho^{2}\right)^{6}} \ddot{\rho}\left(\dot{\rho}^{2}+q \rho^{2}\right)^{2} \\
& -\frac{\Lambda^{4} \dot{\rho}}{4\left(\Lambda_{c}^{2}+\frac{1}{2} \rho^{2}\right)^{6}}\left[2 \dot{\rho} \ddot{\rho}-2 \frac{n^{2}}{r^{3}} \rho^{2}+2 q \rho \dot{\rho}\right]\left(\dot{\rho}^{2}+q \rho^{2}\right) .
\end{aligned}
$$


Inserting equation (8.20) and (8.22) into equation (8.16) gives

$$
\begin{aligned}
0= & -\frac{1}{2} q \rho+\frac{\Lambda^{4} \rho}{8\left(\Lambda_{c}^{2}+\frac{1}{2} \rho^{2}\right)^{7}}\left(\dot{\rho}^{2}+q \rho^{2}\right)^{3}-\frac{\Lambda^{4}}{8\left(\Lambda_{c}^{2}+\frac{1}{2} \rho^{2}\right)^{6}} q \rho\left(\dot{\rho}^{2}+q \rho^{2}\right)^{2} \\
& +\frac{1}{2} \ddot{\rho}-\frac{3 \Lambda^{4} \rho \dot{\rho}^{2}}{4\left(\Lambda_{c}^{2}+\frac{1}{2} \rho^{2}\right)^{7}}\left(\dot{\rho}^{2}+q \rho^{2}\right)^{2}+\frac{\Lambda^{4}}{8\left(\Lambda_{c}^{2}+\frac{1}{2} \rho^{2}\right)^{6}} \ddot{\rho}\left(\dot{\rho}^{2}+q \rho^{2}\right)^{2} \\
& +\frac{\Lambda^{4} \dot{\rho}}{4\left(\Lambda_{c}^{2}+\frac{1}{2} \rho^{2}\right)^{6}}\left[2 \ddot{\rho} \ddot{\rho}-2 \frac{n^{2}}{r^{3}} \rho^{2}+2 q \rho \dot{\rho}\right]\left(\dot{\rho}^{2}+q \rho^{2}\right),
\end{aligned}
$$

which when solved for $\ddot{\rho}$ gives

$$
\ddot{\rho}=\frac{f(r, \rho, \dot{\rho})}{g(r, \rho, \dot{\rho})},
$$

where

$$
\begin{aligned}
f(r, \rho, \dot{\rho})= & 5 \rho \dot{\rho}^{6}+9 q \rho^{3} \dot{\rho}^{4}+3 q^{2} \rho^{5} \dot{\rho}^{2}-q^{3} \rho^{7}-\left(\Lambda_{c}+\frac{1}{2} \rho^{2}\right)\left[-\frac{4 q}{\Lambda^{4}} \rho\left(\Lambda_{c}+\frac{1}{2} \rho^{2}\right)^{6}\right. \\
& \left.+3 q \rho \dot{\rho}^{4}+2 q^{2} \rho^{3} \dot{\rho}^{2}-q^{3} \rho^{5}-4 \rho^{2} \dot{\rho} \frac{n^{2}}{r^{3}}\left(\dot{\rho}^{2}+q \rho^{2}\right)\right] \\
g(r, \rho, \dot{\rho})= & \left(\Lambda_{c}+\frac{1}{2} \rho^{2}\right)\left[\frac{4}{\Lambda^{4}}\left(\Lambda_{c}+\frac{1}{2} \rho^{2}\right)^{6}+5 \dot{\rho}^{4}+6 q \rho^{2} \dot{\rho}^{2}+q^{2} \rho^{4}\right] .
\end{aligned}
$$

To make the expression easier to work with, we will write it on a unitless form. To do so, we introduce the unitless variables

$$
R=\rho / \rho_{0} \quad \text { and } \quad \eta=\rho_{0} \mathrm{r},
$$

similar to those presented in equation (4.29). In this case, we also want $\rho_{0}$ to be the solution to the Euler-Lagrange equations with respect to $\rho$ when $\nabla \rho=\nabla \psi=0$. If we consider the full Lagrangian, it is quite difficult to reach a simple expression for $\rho_{0}$. Instead, we will therefore consider the case $\Lambda_{c}^{2} \ll|\phi|^{2}$, defined as the MOND regime in [1]. This is equivalent to putting $\Lambda_{c}=0$ in our equations, and we get

$$
\begin{aligned}
\mathscr{L}= & -\frac{1}{4}\left(g^{\mu \nu} \partial_{\mu} \rho \partial_{\nu} \rho+g^{\mu \nu} \rho^{2} \partial_{\mu} \psi \partial_{\nu} \psi+m^{2} \rho^{2}\right) \\
& -\frac{\Lambda^{4}}{48\left(\Lambda_{c}^{2}+\frac{1}{2} \rho^{2}\right)^{6}}\left(g^{\mu \nu} \partial_{\mu} \rho \partial_{\nu} \rho+g^{\mu \nu} \rho^{2} \partial_{\mu} \psi \partial_{\nu} \psi+m^{2} \rho^{2}\right)^{3} \\
= & -\frac{1}{4}\left(-\rho^{2} \mu^{2}+m^{2} \rho^{2}\right)-\frac{4 \Lambda^{4}}{3 \rho^{12}}\left(-\rho^{2} \mu^{2}+m^{2} \rho^{2}\right)^{3} \\
= & -\frac{1}{4}\left(m^{2}-\mu^{2}\right) \rho^{2}-\frac{4 \Lambda^{4}}{3}\left(m^{2}-\mu^{2}\right)^{3} \frac{1}{\rho^{6}}
\end{aligned}
$$


which results in the Euler-Lagrange equation

$$
\begin{aligned}
0 & =\frac{\partial \mathscr{L}}{\partial \rho} \\
0 & =-\frac{1}{2}\left(m^{2}-\mu^{2}\right) \rho+8 \Lambda^{4}\left(m^{2}-\mu^{2}\right)^{3} \frac{1}{\rho^{7}} \\
\rho^{8} & =16 \Lambda^{4}\left(m^{2}-\mu^{2}\right)^{2} \\
\rho & =\sqrt{2 \Lambda}\left(m^{2}-\mu^{2}\right)^{1 / 4},
\end{aligned}
$$

meaning that

$$
\rho_{0}=\sqrt{2 \Lambda}\left(m^{2}-\mu^{2}\right)^{1 / 4} .
$$

To calculate the chemical potential we use equation (48) in [1],

$$
\mu=\frac{\rho_{\text {center }}^{2}}{8 \Lambda^{2} m^{5}}
$$

where $\rho_{\text {center }}$ denotes the central density of the condensate (6.19). Inserting the new variables into equation (8.24) gives

$$
\ddot{R}=\frac{\bar{f}(\eta, R, \dot{R})}{\bar{g}(\eta, R, \dot{R})},
$$

with

$$
\begin{aligned}
\bar{f}(\eta, R, \dot{R})= & \rho_{0}^{2}\left(5 R \dot{R}^{6}+9 p R^{3} \dot{R}^{4}+3 p^{2} R^{5} \dot{R}^{2}-p^{3} R^{7}\right) \\
& -\left(\Lambda_{c}^{2}+\frac{1}{2} \rho_{0}^{2} R^{2}\right)\left[-\frac{4 p}{\Lambda^{4} \rho_{0}^{8}} R\left(\Lambda_{c}^{2}+\frac{1}{2} \rho_{0}^{2} R^{2}\right)^{6}\right. \\
& \left.+3 p R \dot{R}^{4}+2 p^{2} R^{3} \dot{R}^{2}-p^{3} R^{5}-4 R^{2} \dot{R} \frac{n^{2}}{\eta^{3}}\left(\dot{R}^{2}+p R^{2}\right)\right] \\
\bar{g}(\eta, R, \dot{R})= & \left(\Lambda_{c}^{2}+\frac{1}{2} \rho_{0}^{2} R^{2}\right)\left[\frac{4}{\Lambda^{4} \rho_{0}^{8}}\left(\Lambda_{c}^{2}+\frac{1}{2} \rho_{0}^{2} R^{2}\right)^{6}+5 \dot{R}^{4}+6 p R^{2} \dot{R}^{2}+p^{2} R^{4}\right] .
\end{aligned}
$$

Here we have defined

$$
p=\frac{\rho_{0}^{2}}{4 \Lambda^{2}}+\frac{n^{2}}{\eta^{2}}
$$

and the " " represents the derivative with respect to $\eta$.

In the following section we will try to interpret equation (8.37) and the behavior of $R(\eta)$. 


\subsection{Interpretation}

Equation (8.37) is a second-order nonlinear ordinary differential equation with associated boundary conditions. Any solution similar to a known vortex solution should have the boundary conditions $R(\eta=0)=0$ and $R(\eta \rightarrow \infty) \rightarrow 1$, as previously seen in section 4.3.1. In other words, the superfluid component of the system must vanish at the center, as Landau's criterion is broken here (the normal component is, however, free to stay). In addition, the vortex will not influence the full superfluid system, and the density far off should be (approximately) the same as for the case without a vortex. As it turns out, finding a vortex solution of equation (8.37) has proved quite difficult. As a result, this section is therefore devoted to investigating the behavior of $R(\eta)$ in the limit of small and large values of $\eta$, or equivalently close and far away from the supposed vortex core. For this we will use the values $m=1 \mathrm{eV}, \Lambda=0.2 \mathrm{meV}$ and $\Lambda_{c}=\Lambda / 2$. Based on the results we get, we will also present an analysis of how different initial values and $\Lambda_{c}$-values impact the solution of the equation.

\subsubsection{Background Information}

Before analyzing the equation of motion resulting from the Lagrangian density given in equation (8.1), we should discuss its origin. The Lagrangian density presented in section 6 of [1] was constructed to reproduce the form of the MOND scalar action in the non-relativistic limit,

$$
\mathscr{L} \sim \Lambda X \sqrt{|X|} .
$$

In the superfluid DM case, it is the phonons that need to be described my the MOND action. In [1], the phonons are characterized by a scalar field $\theta$, which is governed by

$$
X=\frac{d \theta}{d t}-m \phi-\frac{(\nabla \theta)^{2}}{2 m} .
$$

Here $\Phi$ is the external gravitational potential.

In the non-relativistic limit, and the regime $\Lambda_{c} \ll|\phi|$, equation (8.1) has the same form as equation (8.41) (see section 6 of [1]). In other words, our Lagrangian density was constructed with the purpose of reproducing the form of equation (8.41), to ensure that the superfluid phonons mediate a MONDian force when interacting with the baryons. It is therefore unsure whether or not the authors of [1] have made sure that their theory also has a valid vortex solution. They mention that other forms of the Lagrangian density may also result in the desired expression, meaning that the given theory only is a suggestion. Going in to the analysis of equation (8.37), we therefore do not know if the theory has a regular vortex solution, an unusual solution or no solution at all.

\subsubsection{Small $\eta$}

To analyze equation (8.37) in the limit of small $\eta$, or close to the alleged vortex core, we must work out which terms dominate the expression. To do so, we must make some 
assumptions about $R$ and $\dot{R}$ in the given limit. The simplest approach is to assume that a vortex solution exists, and estimate their values based on the approximate vortex solution $R(\eta)=\eta / \sqrt{2+\eta^{2}}$, and its derivative.

We start off by inserting a value $\eta=10^{-9}$, which is equivalent to a radius of $r=\eta / \rho_{0} \sim 10^{-13} \mathrm{~cm}$ out from the core, into equation (8.37). This tells us that the sixth and ninth terms dominate the numerator, while the second term dominates in the denominator. For small $\eta$ we then get an approximate expression

$$
\ddot{R} \sim \frac{-\left(\Lambda_{c}^{2}+\frac{1}{2} \rho_{0}^{2} R^{2}\right)\left(3 p R \dot{R}^{4}-4 R^{2} \dot{R}_{\left.\frac{n^{2}}{\eta^{3}} \dot{R}^{2}\right)}\right.}{\left(\Lambda_{c}^{2}+\frac{1}{2} \rho_{0}^{2} R^{2}\right) 5 \dot{R}^{4}}
$$

for the double derivative of $R$. Contracting and only including the second term in $p$ $\left(p \sim n^{2} / \eta^{2}\right)$, along with inserting $n=1$, we get

$$
\ddot{R} \sim \frac{R}{\eta^{2}}\left(\frac{3}{5}-\frac{4}{5} \frac{R}{\eta \dot{R}}\right)
$$

for small $\eta$. We then solve equation (8.44) numerically by applying a 4th order RungeKutta method, using the values of the approximate vortex solution and its derivative as initial conditions. The solution is illustrated in figure 8.1a and shows an almost linear behavior. So far, this is in accordance with what we expect from a regular vortex solution.

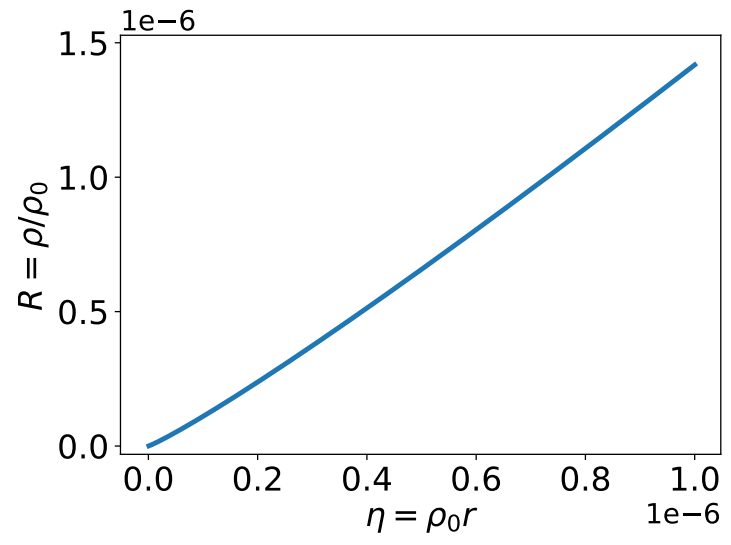

(a)

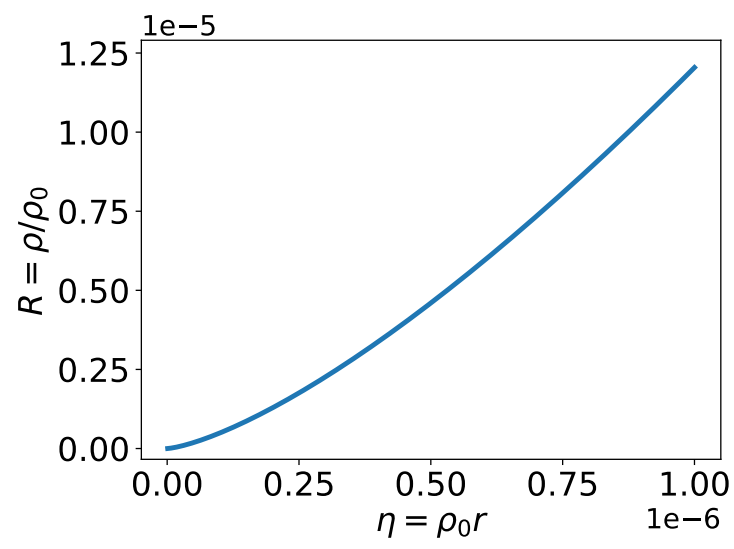

(b)

Figure 8.1: Figure (a) shows the solution of equation (8.37) for very small values of $\eta$, using the approximate vortex solution $\left(R=\eta / \sqrt{2+\eta^{2}}\right)$ to decide the initial conditions. Figure (b) again shows the solution close to the core, but now using $R=\eta$ and $\dot{R}=1$ as initial conditions. The form of the solutions are similar in the two cases, although more curved for the second case. Both present a plausible vortex profile close to the core. 
It might be the case that there is no vortex solution, or that the vortex solution of this model has a form that is unfamiliar to us. In that case, we would also like to check the limit of small $\eta$ without using the approximate vortex solution as an initial condition. Let us assume a linear relation for small $\eta$, so that $\eta=R$, and a value $\dot{R} \gg R$. In this case, it is the ninth and tenth terms that dominate the numerator, and the second and third term in the denominator. We then get an expression

$$
\ddot{R} \sim \frac{4 R^{2} \dot{R}^{2} \eta^{2}+4 R^{4}}{5 \dot{R}^{3} \eta^{5}+6 R^{2} \dot{R} \eta^{3}}
$$

if we again insert $n=1$ and $p \sim n^{2} / \eta^{2}$. Solving this the same way as before, we get the solution illustrated in figure 8.1b. Again we had $\eta=10^{-9}$ as our starting point, which for $R=\eta$ gave a slightly higher initial value for $R$ than the approximate vortex solution estimate. We also chose a value of $\dot{R}=1$, while the approximate vortex solution gave $\dot{R} \sim 1$.3. From the plot we see that this results in a slightly more curved result than the other, although with opposite curvature of the original vortex equation, illustrated in figure 4.3. This is, however, still a plausible start for a vortex profile. Yet, if we go to small enough x-values, any increasing function could look almost linear. The behavior for large $\eta$ is therefore maybe the most interesting for us to study.

\subsubsection{Large $\eta$}

For a regular vortex equation, we expect the resulting profile to converge towards a constant value far from the vortex. This limit is therefore an important part of our analysis. Far away, we should be in the regime of $\Lambda_{c} \ll|\phi|$, which, as shown in section 6 of [1], is the MOND regime of the theory. We may therefore ignore $\Lambda_{c}$ in equation (8.37).

Let us do as before, and first assume that a vortex solution somewhat similar to the regular one exists. In this way we may use the approximate vortex solution to estimate the values of the different terms in equation (8.37) far from the vortex center. In this case, we are solving the equation for central density values, meaning that we should choose a radius larger than $10^{-4} \mathrm{~m}$, that is $\eta \approx 10$, as this is the vortex core size at the center of a galaxy for the case of superfluid DM particles of $1 \mathrm{eV}$ mass. Based on this, we set $\eta=200$, which should be well outside the central vortex core. Inserting the resulting values of $R$ and $\dot{R}$ into equation (8.37), we find that it is the fourth, fifth and eight term that dominates the numerator, and the first and fourth that dominates the denominator. Only keeping these terms, and letting $p \rightarrow \frac{\rho_{0}^{2}}{4 \Lambda^{2}}$, results in

$$
\ddot{R} \sim \frac{\rho_{0}^{2}}{4 \Lambda^{2}} R
$$

Immediately we can see that this is an exponential profile, and solving from $\eta=200$ and out, with the corresponding values of $R$ and $\dot{R}$ from the approximate vortex solution, we get the result shown in figure $8.2 \mathrm{a}$. In other words, we get an exponential growth which quickly builds up to a numerical overflow. 


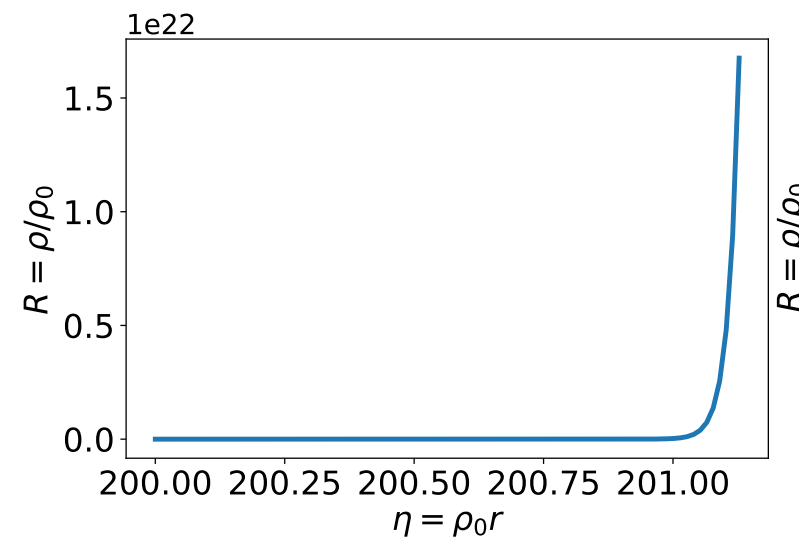

(a)

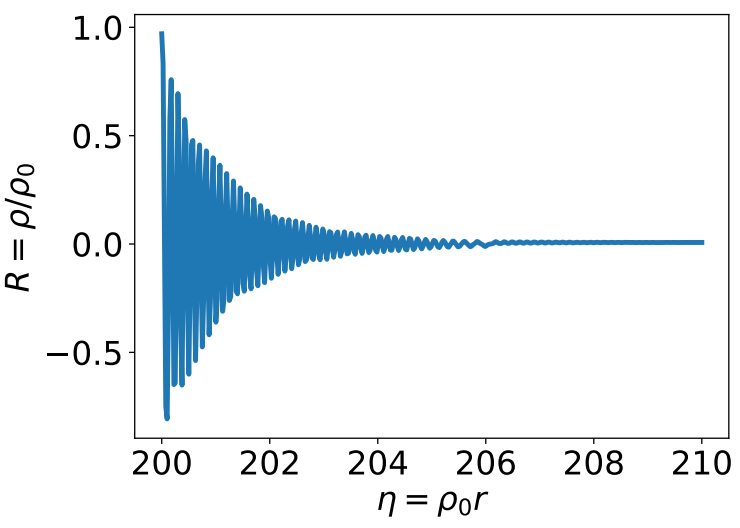

(b)

Figure 8.2: Figure (a) shows the solution of equation (8.37) for large values of $\eta$, using the approximate vortex solution $\left(R=\eta / \sqrt{2+\eta^{2}}\right)$ to decide the initial conditions. Figure (b) again shows the solution far from the core, but now using $R=0.97$ and $\dot{R}=10^{-6}$ as initial conditions. The form of the solutions are completely different in the two cases, although none of them represent a realistic vortex profile far from the core.

To obtain the expression in equation (8.46), we used the approximation $p \rightarrow \frac{\rho_{0}^{2}}{4 \Lambda^{2}}$ for large $\eta$. When solving the equation numerically, we may still include the full expression for $p$, which also allows for a different result. If we choose some small value of $\dot{R}$ as our initial value (far off from the center) and then choose $R$ to be a value below one, for example $R=0.97$, we may get solutions on the form presented in figure $8.2 \mathrm{~b}$. This solution is oscillating around zero initially, and then gradually settles down to a constant value slightly above zero. Although this solution is quite different from the exponential solution, it is still not a plausible vortex profile ${ }^{2}$. There are, however, several examples where vortex profiles have oscillations close to the singularity [73, 74], although this is usually a property of very dense superfluids, such as HeII [75]. The superfluid dark matter is a dilute superfluid and is therefore not expected to have this property. However, studying a superfluid in the dark matter picture could bring with it unexpected behavior.

In this section we have seen that the vortex equation (8.37) is quite unstable at large $\eta$, and any vortex solution resembling the one in section 4.3.1 seems improbable. However, to obtain the solutions in figure 8.2 we had to choose some initial values of $R$ and $\dot{R}$. For the plot in figure $8.2 \mathrm{~b}$, we had to change initial conditions away from the approximate vortex solution ones to obtain a result that did not immediately overflow. This tells us that the vortex equation is very sensitive to the choice of initial conditions, which should be investigated further. This is done in the following section.

\footnotetext{
${ }^{2}$ If this is supposed to be a density profile, values below zero does not make much sense.
} 


\subsubsection{Numerical Stability}

We have now analyzed equation (8.37) in different limits, focusing on the dominant terms in each case. If we also look at the limit $\eta \rightarrow \infty$, and assume that the solution behaves as expected, we should have $\dot{R} \rightarrow 0$ and $R \rightarrow 1$. Inserting this into equation (8.38) and (8.39), along with $p \rightarrow \frac{\rho_{0}^{2}}{4 \Lambda^{2}}$, and keeping all terms, we get:

$$
\begin{aligned}
& \bar{f} \rightarrow-\rho_{0}^{2} \frac{\rho_{0}^{6}}{4^{3} \Lambda^{6}}-\left(\Lambda_{c}^{2}+\frac{1}{2} \rho_{0}^{2}\right)\left[-\frac{4 \frac{\rho_{0}^{2}}{4 \Lambda^{2}}}{\Lambda^{4} \rho_{0}^{8}}\left(\Lambda_{c}^{2}+\frac{1}{2} \rho_{0}^{2}\right)^{6}-\frac{\rho_{0}^{6}}{4^{3} \Lambda^{6}}\right], \\
& \bar{g} \rightarrow\left(\Lambda_{c}^{2}+\frac{1}{2} \rho_{0}^{2}\right)\left[\frac{4}{\Lambda^{4} \rho_{0}^{8}}\left(\Lambda_{c}^{2}+\frac{1}{2} \rho_{0}^{2}\right)^{6}+\frac{\rho_{0}^{4}}{4^{2} \Lambda^{4}}\right] .
\end{aligned}
$$

In this limit we should also have $\Lambda_{c} \ll|\phi|$, which from earlier means that we can ignore $\Lambda_{c}$ in our expressions. This gives

$$
\begin{aligned}
& \bar{f} \rightarrow-\frac{\rho_{0}^{8}}{2^{6} \Lambda^{6}}-\frac{1}{2} \rho_{0}^{2}\left[-\frac{1}{\Lambda^{6} \rho_{0}^{6}} \frac{1}{2^{6}} \rho_{0}^{12}-\frac{\rho_{0}^{6}}{2^{6} \Lambda^{6}}\right]=-\frac{\rho_{0}^{8}}{2^{6} \Lambda^{6}}+\frac{\rho_{0}^{8}}{2^{6} \Lambda^{6}}=0, \\
& \bar{g} \rightarrow \frac{1}{2} \rho_{0}^{2}\left[\frac{4}{\Lambda^{4} \rho_{0}^{8}} \frac{1}{2^{6}} \rho_{0}^{12}+\frac{\rho_{0}^{4}}{4^{2} \Lambda^{4}}\right]=\frac{\rho_{0}^{6}}{2^{5} \Lambda^{4}}+\frac{\rho_{0}^{6}}{2^{5} \Lambda^{4}}=\frac{\rho_{0}^{6}}{2^{4} \Lambda^{4}},
\end{aligned}
$$

which is self-consistent and indicates that there might be some solution after all. Because of this, we also tried to solve equation (8.37) directly, using a variety of methods (see appendix B). One of these methods is the shooting method. Here you guess at one of the initial conditions of your boundary value problem, solve the equation like a regular initial value problem and then check if the last value is far from the given boundary condition. If the value is within a given tolerance, your shot was successful. If not, you make a new guess and try again. In section 8.3.2 and 8.3.3 we guessed at initial values somewhat similar to what we expect from a regular vortex profile. This did not result in any viable vortex solutions for the superfluid DM vortex equation. Still, any vortex profile should at least fulfill the boundary conditions of $\rho(r=0)=0$ and $\rho(r \rightarrow \infty) \rightarrow$ constant. The shooting method should therefore be able to solve our equation, as we can check if our result is close to a constant value for large $\eta$, and if not, just make a new guess. The problem with this, however, is demonstrated in figure 8.2a. For many initial value guesses, the ordinary differential equation (ODE) solver will immediately result in overflow, thereby removing the chance to check if the last point is close to the boundary condition, and from that make a new initial value guess.

In this section we therefore want to analyze the stability of equation (8.37), to see if we can reach any conclusion regarding the nature of the solution ${ }^{3}$. As we have seen, equation (8.37) is quite unstable, and depends heavily on the initial conditions of $\dot{R}$. In addition, we have the parameter $\Lambda_{c}$, which is not decided in [1]. We also have the parameters $m$ and $\Lambda$, which from chapter 6 can take many values. We will, however, stick with our original parameters (equation 6.21) for this analysis. Based on this, we

\footnotetext{
${ }^{3}$ The idea and numerical code behind the results of this section originates from Robert Hagala [76], a Ph.D. research fellow at the Institute of Theoretical Astrophysics at the University of Oslo.
} 
will therefore, for various values of $\Lambda_{c}$, make several initial guesses of $\dot{R}$ and check if there are any regions in parameter space that might allow for a vortex solution with the boundary conditions $R(\eta=0)=0$ and $R(\eta \rightarrow \infty) \rightarrow 1$. Figure 8.3 shows the results of our shooting attempts for different values of $\Lambda_{c}$ and $\dot{R}$.

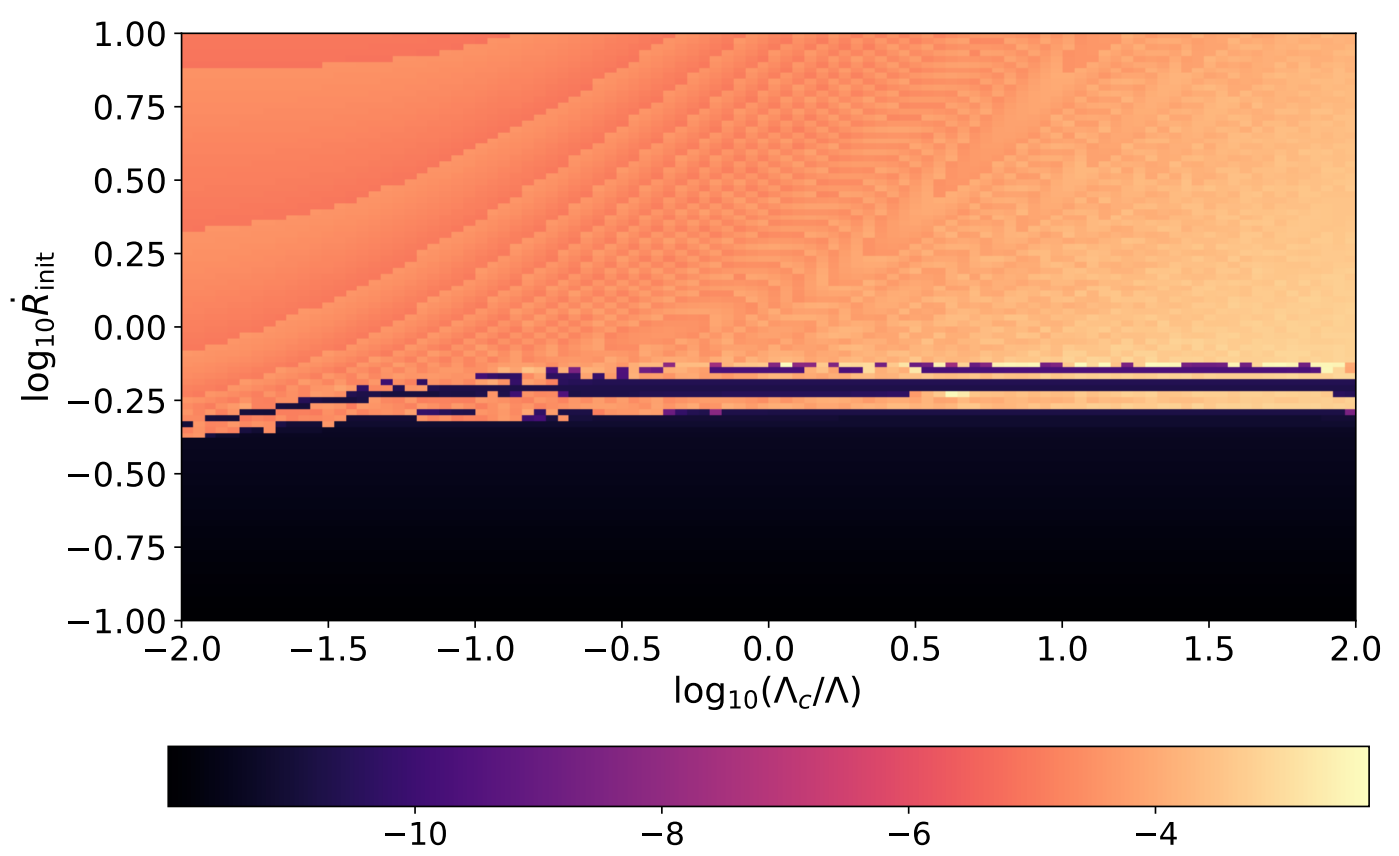

Figure 8.3: Grid showing how "good" a shot is, based on how many iterations are completed without the value of $R$ exceeding $R=2$. The equations have been normalized so that the value of $R$ should stabilize at one, however, we allow some higher values to make sure that we include solutions that overshoot in the beginning, but later stabilize. How "well" a shot has done is indicated by a color gradient, moving from black to white. The scale is logarithmic, meaning that 0 would indicate a solution that has converged to the given boundary values, while the size of the negative values indicate how far along the $\eta$-axis the completed iterations take us, before surpassing $R=2$ or diverging in some way or another. The solutions are weighted by the number $(i / N) \times R_{\max }$, where $i$ is the number of iterations reached, $N$ is the total number of iterations and $R_{\max }$ is the maximum value of the solution. A good shot would have $i \approx N$ and $R_{\max } \approx 1$, so that the total weight is close to 1 . It is the logarithm of this value that is displayed in the colorbar at the bottom.

From figure 8.3 it is evident that most values diverge in one way or another. The black parameter combinations show solutions that diverge from $R(\eta=0)=0$ and $R(\eta \rightarrow \infty) \rightarrow 1$ right away, while the orange points "survive" a higher quantity of iterations before diverging. A few yellow points may be observed in the $\log _{10} \dot{R} \in$ $[-0.3,0.0]$ region, for values of $\log _{10}\left(\Lambda_{c} / \Lambda\right) \in[0.0,2.0]$. These are interesting, as they 
represent shootings that could provide vortex-like solutions. However, observational constraints on the MOND regime result in an upper limit of $\Lambda_{c}$, given as

$$
\frac{\Lambda_{c}}{\Lambda} \lesssim \frac{\alpha^{2}}{10}
$$

This may be found in section 6 of [1]. For our value of $\Lambda=0.2 \mathrm{meV}$, we found the value $\alpha \approx 2.51$ in section 6.3 , which again results in the limit $\Lambda_{c} / \Lambda \lesssim 0.63$. This means that all the points above $\log _{10}\left(\Lambda_{c} / \Lambda\right) \approx-0.2$ in figure 8.3 are ruled out by observations. This region is also where we find most of our plausible solutions to the vortex equation. Figure 8.4 illustrates the area we are left with.

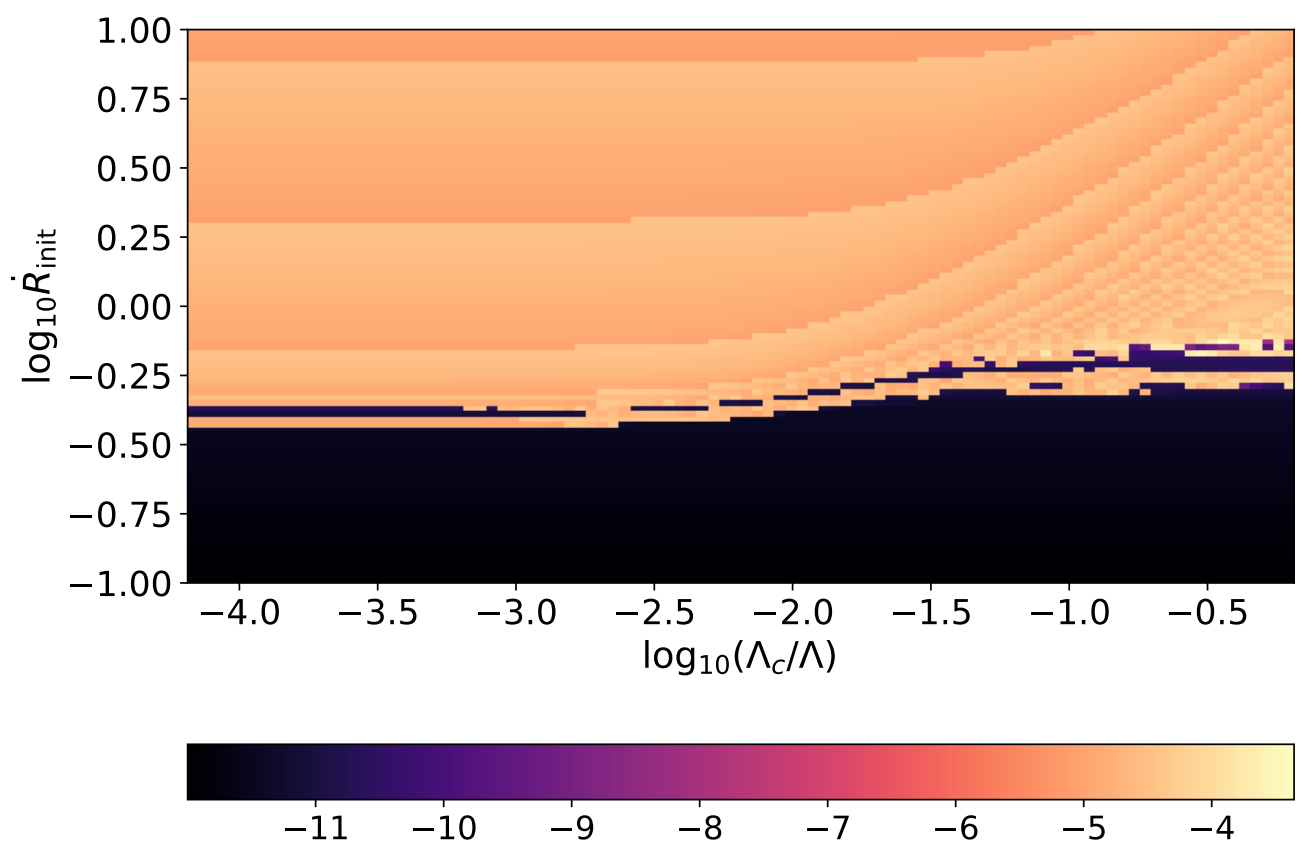

Figure 8.4: Remake of figure 8.3, excluding values of $\Lambda_{c}$ that have been ruled out by observations. The most promising region of figure 8.3 is now gone, leaving us with a small area of plausible parameter combinations for $\log _{10} \dot{R} \in[-0.3,0.0]$ and $\log _{10}\left(\Lambda_{c} / \Lambda\right) \in[-1.0,0.0]$.

After investigating the region $\log _{10} \dot{R} \in[-0.3,0.0]$ and $\log _{10}\left(\Lambda_{c} / \Lambda\right) \in[-1.0,0.0]$ up close, we find that the area $\log _{10} \dot{R} \in[-0.25,-0.15]$ and $\log _{10}\left(\Lambda_{c} / \Lambda\right) \in[-1.15,-0.95]$ hold some of the most promising results. In figure 8.5, we show the specific region up close. From the figure we observe that most of the shootings still fail to give a result that upholds the given boundary conditions, and that the region is highly unstable. Still, there is a small number of light yellow points, some of them reaching values close to " 0 " on the logarithmic scale. This does still mean that the parameter combinations 

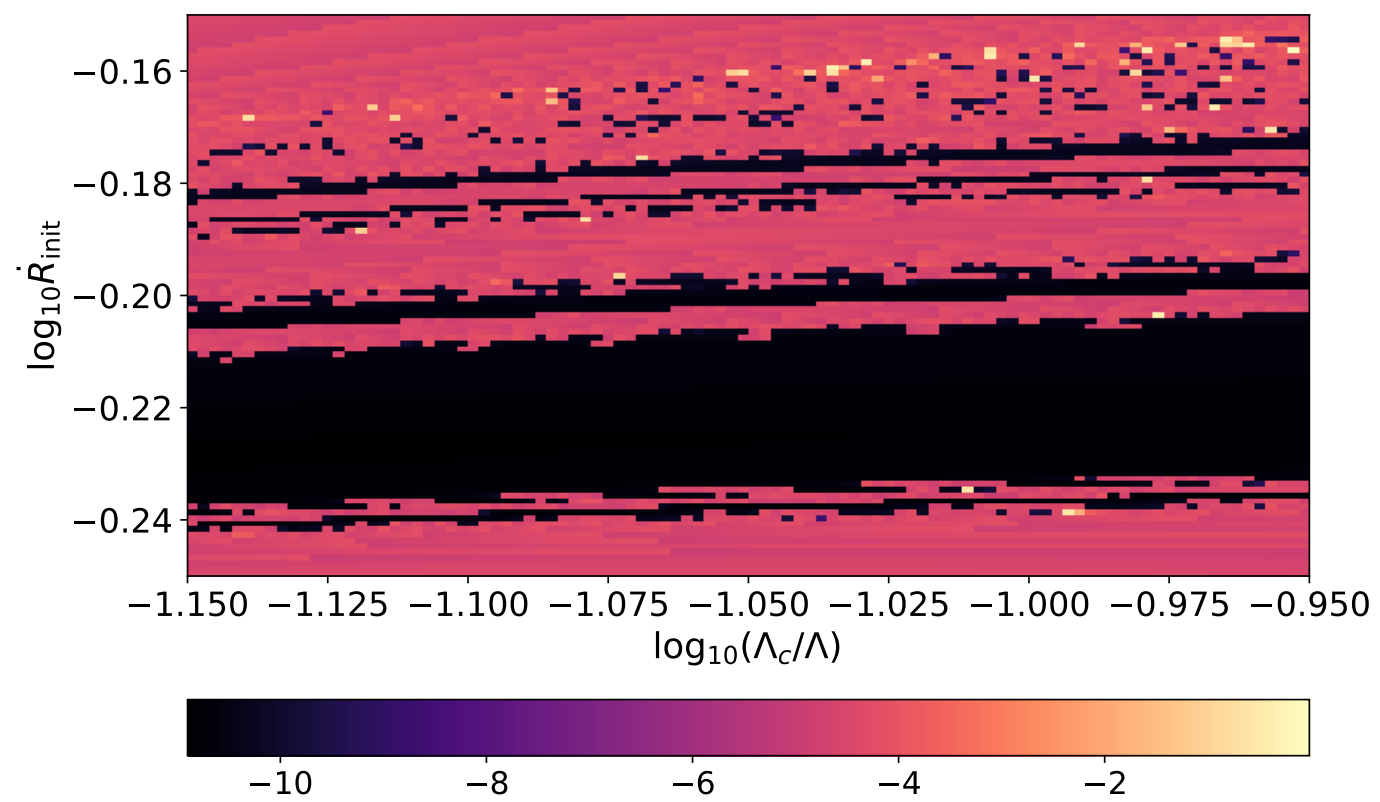

Figure 8.5: More refined grid for the region $\log _{10} \dot{R} \in[-0.25,-0.15], \log _{10}\left(\Lambda_{c} / \Lambda\right) \in$ $[-1.15,-0.95]$ in figure 8.4. It is evident that the given region is highly unstable, and that none of the shootings completely reach the desired results. There are a few yellow points which indicate promising results.

result in a shooting that diverges, but that we have found an area where the shootings "survive" a higher number of iterations. This might indicate that there exists, for extremely fine-tuned values of $\dot{R}_{\text {init }}$ and $\Lambda_{c}$, a vortex solution somewhat resembling what we would expect. However, in general, having to fine-tune any parameters of a model is not desirable.

In figure 8.6, one of the good shots of figure 8.5 is presented. It is clear that what the code regards as a "good" solution in reality is far from what we expect. The vortex profile in figure 8.6 oscillates, as indicated in section 8.3.3, but never reaches values close to $R=1$. The solution also suddenly diverges towards infinity at $\eta \sim 4$. This latter point is not included in the figure in order to better illustrate the behavior for small $\eta$. This means that, even though the code tells us that some "good" solutions exist, they do not necessarily serve as realistic vortex profiles. At least not in the region of parameter space allowed by observations. For larger values of $\Lambda_{c}$, the parameter combinations produce a higher quantity of "good" solutions, and it might be the case that some of these are along the lines of what we are looking for. This is, however, not relevant, as that specific region of parameter space is inaccessible. 


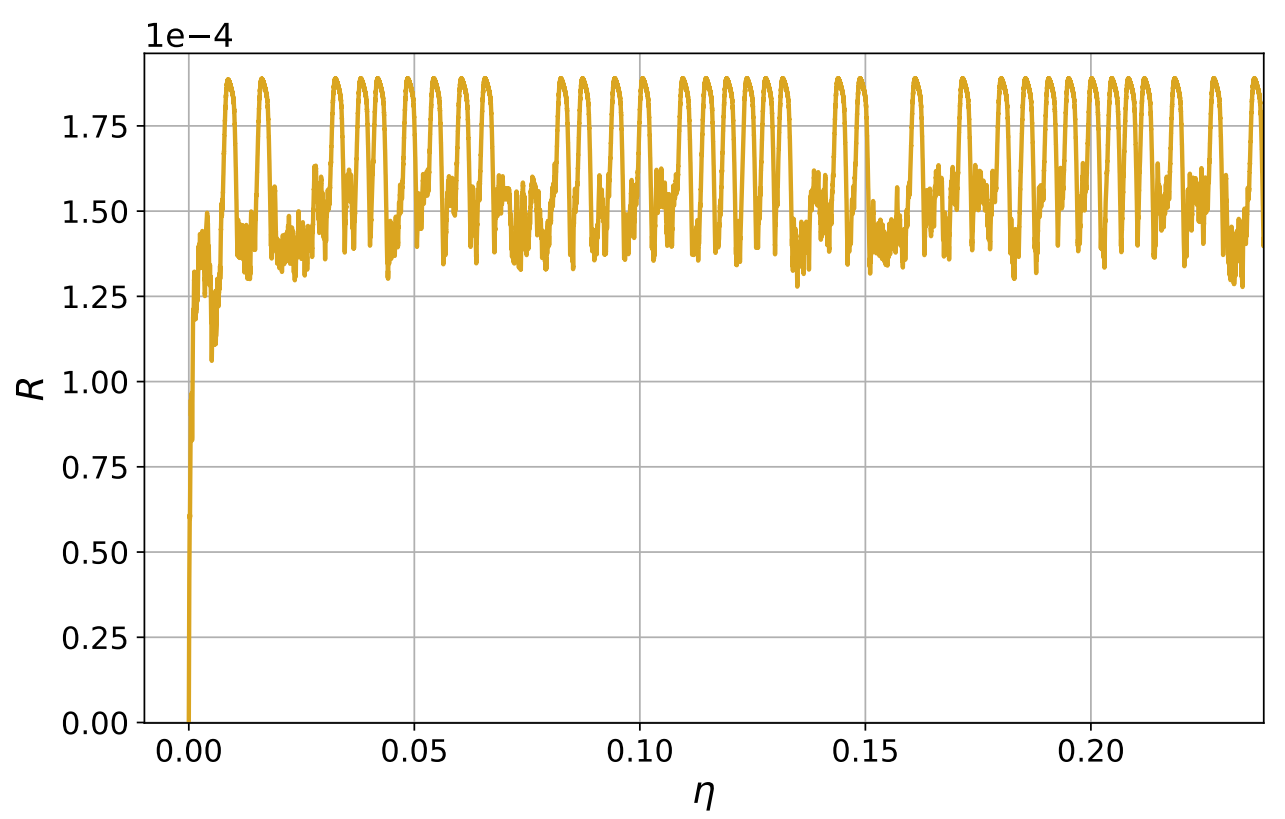

Figure 8.6: Example of a "good" shot from figure 8.5. The plot shows the solution of the vortex equation for $\dot{R}_{\text {init }} \simeq 0.698$ and $\Lambda_{c} \simeq 0.021 \mathrm{meV}$. The vortex profile has an oscillatory behavior, as predicted in section 8.3.3, but will move on to diverge for $\eta \sim 4$. In addition, the solution does not oscillate around $R=1$, but rather $R \sim 1.5 \times 10^{-4}$.

It might be the case that allowing some changes in $m$ and $\Lambda$ (which seems completely viable from chapter 6) could loosen up some of the requirements on $\dot{R}_{\text {init }}$ and $\Lambda_{c}$. This could be something to investigate in the future. The implications of this section will be further discussed in chapter 9 , however, at this point it seems unlikely that the theory presented in section 6 of [1] has a realistic vortex solution. It is possible that some solution could be found for extremely fine-tuned values of $\dot{R}_{\text {init }}$ and $\Lambda_{c}$. Still, there is nothing preventing $\Lambda_{c}$ from varying slightly form galaxy to galaxy, thus again making it unlikely that we have the combination necessary for realistic vortex profiles, at least on a form that we are familiar with. 


\section{Chapter 9}

\section{Discussion}

"Think think think. Words, letters, numbers, lines!"

- The Doctor, Doctor Who: The Shakespeare Code.

It is now time to discuss some of the results obtained in the previous chapters. This will be done chapter by chapter in the sections below. As a reminder, the goal of this thesis was to further investigate the parameters of the superfluid DM model, the nature of vortices and the vortex ability of impacting, or being impacted by, the surroundings.

\subsection{Chapter 5 - A Simple Model}

In this chapter, we calculated some properties of a DM superfluid in a constant density halo $\left(\Phi \propto r^{2}\right)$. As the BEC theory of chapter 3 is based on a HO potential $\left(\propto r^{2}\right)$, we could use our already established knowledge to describe the superfluid.

One of the goals of this thesis was to better understand the nature of the vortices that might form in the superfluid dark matter component of a halo. Although the constant density case is a simplification of reality, we gained some interesting insight from this chapter. Let us first summarize the results in a few points:

1. The angular velocity of a galaxy is way above the critical angular velocity required for vortex formation.

2. The size of a single vortex core is $\sim 1 \mathrm{~mm}$.

3. The intervortex spacing is $\sim 0.002 \mathrm{AU}$.

4. The condensate radius is $\lesssim 83.16 \mathrm{kpc}$.

5. The energy per unit length required to produce a single vortex is $\sim 10^{-13} \mathrm{~g} \mathrm{~cm} / \mathrm{s}^{2}$.

6. The "mass" of a single vortex is $\sim 10^{-14} \mathrm{~kg}$.

7. The dark matter temperature is below the critical temperature. 
Now, let us discuss the above points, one by one:

1. From our calculations we obtain a critical angular velocity of $\Omega_{c} \sim 10^{-39} \mathrm{~s}^{-1}$ and an approximated actual angular velocity of $\sim 10^{-16} \mathrm{~s}^{-1}$. This demonstrates that the angular velocity of a Milky Way-like galaxy is way above the critical angular velocity required for vortex formation. From section 3.3.2 we know that this will result in a grid of vortices, spanning the full condensate component.

In section 10 of [1], Berezhiani and Khoury also present a simple estimate of the critical angular velocity and galaxy angular velocity. The angular velocities we obtain here are about 1-2 orders of magnitude higher that the ones obtained in [1]. This is likely a result of choosing different values to insert in the equations. For example, in [1], a density of $\rho \sim 10^{-25} \mathrm{~g} / \mathrm{cm}^{3}$ was used for the calculations, while we used a density 200 times the present day critical density, of order $\sim 10^{-27} \mathrm{~g} / \mathrm{cm}^{3}$. It is also worth mentioning that the calculations, in both cases, are based upon assumptions and simplifications. Still, they do address what is necessary, namely that the angular velocity of a galaxy such as the Milky Way is way above the critical angular velocity, leading to the conclusion that multiple vortices should exist throughout the galaxy.

2. In compliance with section 10 of [1], we find that the size of a single vortex core is $\sim 1 \mathrm{~mm}$. Let us take a moment to appreciate how incredibly small this is. From point three we know that the intervortex spacing of the vortex grid in the superfluid component is $0.002 \mathrm{AU}$. The distance between the Earth and the Moon is roughly $0.009 \mathrm{AU}$, which means that the relative size and distance between two vortices is the same as the distance between a single flea on Earth and a single flea on the Moon.

3. As also mentioned in the point above, we found that the vortices in a grid covering the whole condensate component will be separated by roughly $\sim 0.002 \mathrm{AU}$. This is based on the assumption of a uniform grid which mimics rigid body rotation. However, a galaxy does not rotate uniformly, which might also be the case for the DM halo. An experiment carried out in [61], shows that vortices formed in a condensate that does not rotate uniformly have a tendency to move towards the edge of the condensate in a ring formation. If there is any interaction between the vortices and the baryons, a ring formation might affect the overall rotation of the baryons differently than a uniform grid.

4. Some of our calculations required the condensate radius, which we estimated based on equation (22) in [11]. This resulted in an upper limit of $83.16 \mathrm{kpc}$, which is the value we used for our calculations. It should be noted that choosing a lower value here would have resulted in a somewhat larger vortex core size, but as we have to keep the condensate radius above $60 \mathrm{kpc}$ to make sure that the model can explain rotation curves, the size would still be of the same scale. 
5. We also calculated the energy per unit length (in two dimensions) required to produce a single vortex, which was not very high $\left(\sim 10^{-13} \mathrm{~g} \mathrm{~cm} / \mathrm{s}^{2}\right)$. This is, however, exactly the point. For a rotating superfluid, producing vortices results in the lowest energy outcome, which is the reason why we expect to have this vortex grid throughout the condensate.

6. Taking the above point one step further, we also calculated the three-dimensional energy of a single vortex at the center of the condensate. This resulted in a value $\sim 10^{3} \mathrm{~J}$, which when converted to a mass gives approximately $10^{-14} \mathrm{~kg}$. This number is interesting, as it gives an indication of the gravitational effect one single vortex would have on its surroundings. Not surprisingly, this number is very small. Not even adding the mass of all the vortices together amounts to anything substantial, and although this chapter represents a huge simplification of the actual situation, the magnitude of the numbers obtained will give us some sort of indication regarding the vortices and their importance. So far, using parameters of the scales proposed in $[1,11]$, the vortices seem rather insignificant.

7. At the very end, we used the virial theorem to estimate the temperature of the dark matter. As we mentioned, discussing the temperature of dark matter might be a bit odd, as we are used to viewing the particles as collisionless. For a thermodynamic temperature to be well defined, we need the system to be in thermal equilibrium, which requires som form of interaction. In this model, the dark matter particles do self-interact, and they have to thermalize in order to form a condensate. This means that the dark matter should have a temperature, which is the one we calculate using the virial theorem. We also calculated the critical temperature of the system, which is the temperature that defines the transition from a regular state to a condensate state. This calculation is again based on the equations obtained when using a harmonic oscillator potential, and we get that the dark matter temperature indeed is below the critical temperature, which agrees with the formation of a condensate.

All in all, this chapter addresses the thesis goal of better understanding the superfluid and its vortices. We learn that the dark matter is cold enough to form a superfluid, that the halo rotates fast enough that vortices will form and we even obtain an estimate of the size and distribution of vortices throughout the superfluid component. Another goal of this thesis was to investigate the impact the vortices might have on their surroundings. At this point, based on their size and distribution alone, it seems unlikely that the vortices can affect any neighbouring baryons. 


\subsection{Chapter 6 - Parameter Variation}

In this chapter we investigated what would happen if we changed the parameters $m$ and $\Lambda$ of the superfluid DM model. This was inspired by the results of the constant density DM halo consideration, where we found that the vortex cores were $\sim \mathrm{mm}$ in size, and that the "mass" of a single vortex was of the order $10^{-14} \mathrm{~kg}$. One of the goals of this thesis was to investigate the impact of the vortices. Intuitively it seems more likely that the vortices will have an effect on their surroundings if they are bigger. However, the energy expression in equation (5.30), at first glance, seems to say otherwise. When looking closer, we found that a lower particle DM mass would result in a larger energy per vortex, and at the same time a larger vortex core. From this we started investigating what needed change in order to have smaller particle masses, and thus larger vortex cores, and whether these changes would affect the viability of the model. Of course, we are still talking about dark matter, which should not interact greatly with baryons through any other means than gravity. However, the dark matter particles might drag the baryons along with the vortex movement somehow, which seems more likely if the movement is big, and the vortices have a greater "mass." In addition, there have been models proposing a Bose-Einstein condensate DM before, one of which have had vortex cores on $\mathrm{kpc}$ scale $[77]^{1}$. This makes the small vortices of the current model interesting, and a further investigation into a possible growth of size is therefore due. In addition, it is also interesting in itself to check how the model responds to changes in the parameters.

Again we will summarize the results of the chapter in a few points:

1. The zero-temperature condensate density profile was calculated.

2. Values of $m$ and $\Lambda$ which resulted in a one meter vortex core was found.

3. The $\alpha$ parameter of the model was addressed.

4. The impact of our parameter changes on galactic rotation curves was investigated.

5. A lower limit of the DM particle mass was considered.

Let us now discuss the points, one by one:

1. We started off my calculating a zero temperature density profile for the condensate component. Calculating the condensate profile gave us a new way of estimating the condensate radius, namely the value where the density hits zero. For a Milky Way-like galaxy, we found this to be around $84.7 \mathrm{kpc}$, which is only slightly larger than what we found in the constant density case, and above the $60 \mathrm{kpc}$ requirement imposed in [11] to make sure that the model explains flat rotation curves.

\footnotetext{
${ }^{1}$ This model does, however, consider two-body interactions, as opposed to our three-body interactions, necessary to obtain the MONDian force mediated by the phonons. In addition, they consider a mass of $\sim 10^{-22} \mathrm{eV}$, which for us would lead to a huge $\Lambda$-value, way above the Planck mass scale. Ignoring this, the low DM mass would result in a vortex core size of $\sim 0.03 \mathrm{kpc}$ in the center and $\sim 0.5$ $\mathrm{kpc}$ at the edge, which is not far from the scales obtained in the given article.
} 
The density profile calculation was based upon section 3.2 in [1], and it presents a simplified picture of the actual distribution. In reality, the dark matter is not at an absolute zero temperature, and finite temperature effects must be taken into account. In addition, the calculation does not consider the gravitational effect the surrounding baryons would have on the dark matter, which should be addressed for a more realistic profile. The finite temperature effects, and the effect of the baryons, were included in the density profile calculation presented in [11], which resulted in a new estimate of the condensate radius (equation 5.17). This new consideration was, however, quite advanced, and for our simple parameter variations we stayed with our original condensate profile. Still, we chose the value of $\Lambda$ carefully, so that the upper bound on the thermalization radius found from equation (5.17) closely match the value found by where the simplified condensate profile hits zero. In general, the new consideration, leading to the thermalization radius expression, results in smaller condensate components. This could affect the possibilities of observing the vortices directly, through for example lensing.

One of the other consequences of operating with a density profile, instead of a constant value, was that the vortex core size now varied throughout the condensate component. From this we discovered that the millimeter scale was more correct for the edge of the condensate, while the center had even smaller cores. If we compare to the more accurate density profile obtained in [11], which have a higher density at the center of the condensate compared to the zero-temperature case, we would get even smaller vortex cores at the center. To again appreciate this new central vortex size, we may imagine two individual mist water particles, roughly separated by the Earth-Moon distance.

2. Upon realizing how small and insignificant the vortices were, we wanted to check if the superfluid dark matter model allowed for changes in the parameters that would lead to vortices of larger "masses," and thus greater gravitational impact. To do so, we lowered the mass of the dark matter particle, and altered $\Lambda$ accordingly to reproduce the original density profile (point one). To have a specific goal in mind, we chose particle mass values that would create a one meter vortex core. As the density varies throughout the condensate, different masses would be required to produce one meter vortex cores at different locations. We therefore decided to find a particle mass that would give us a one meter vortex core in the center of the condensate, and another mass that would give us a one meter vortex core at the edge.

We found that a DM particle mass of $\sim 10^{-4} \mathrm{eV}$ was needed to obtain a one meter vortex core at the center of the condensate. To achieve the same at the edge, we found that a mass of $\sim \mathrm{meV}$ would be required. After calculating a new mass, we tried to change the value of $\Lambda$ accordingly, so as to reproduce the original density profile. This was done as the original density profile was created with parameter values closed to the scales proposed in $[1,11]$, and by reproducing it we made sure that the profile stayed somewhat realistic. A small deviation in the density values 
should be fine, but staying close to the already established profile was the safest way to estimate the parameters. For the center case we found that a $\Lambda$-value of around $\sim 10^{8} \mathrm{eV}$ would replicate the original profile, and for the edge, a value of $\sim 10^{4} \mathrm{eV}$ was needed. These values are quite the change from the meV scales originally suggested in $[1,11]$, but still seem to satisfy all constrains presented in the aforementioned papers. We should point out that the expression used to calculate the size of the vortex core was the coherence length expression from chapter 3 . As you might remember, this was based on a harmonic oscillator trap, which we replicated by a constant density halo. In this case, however, the density is not constant anymore. Still, as the vortices are small, the density may be considered constant in the vicinity of a vortex, and the expression may still be used.

From the new values of $m$ and $\Lambda$, we further found a cross section of the size $\sigma / m \sim 10^{-18} \mathrm{~cm}^{2} / \mathrm{g}$ for the center case and $\sigma / m \sim 10^{-13} \mathrm{~cm}^{2} / \mathrm{g}$ for the edge, which suggest that the DM particles self-interact quite weakly. Observational constraints from galaxy cluster mergers only put an upper limit on the DM self-interaction [78], namely $\sigma / m \lesssim 0.5 \mathrm{~cm}^{2} / \mathrm{g}$, which we are well below. There is, however, an unknown connection that has not been taken into account here. In section II of [11] it is mentioned that $\Lambda$ might be related to the self-interaction strength of the DM particles ${ }^{2}$. In other words, there might be some relation, yet to be specified, between $\Lambda$ and $\sigma / m$, which could change the result of our parameter variations. When we decrease the mass, $\Lambda$ increases and $\sigma / m$ decreases (significantly), and if there is a relation between these two, stretching their values in opposite directions like this, might be problematic. Still, after discussion with Benoit Famaey [79] (one of the authors of [11]), the conclusion was that increasing $\Lambda$ and decreasing $\sigma / m$ should be acceptable within the constraints set by the model. There is also the fact that the upper limit resulting from the galaxy cluster merger survey was calculated based on standard DM particles, and not a two-component scenario as would be necessary in this framework. This might change the merger constraints.

We also addressed some other values, such as the critical temperature, the critical angular velocity and again the "mass" of a single vortex. We found the critical temperature and angular velocity to be within the expectations for formation of a BEC, for both the new masses. These calculations were, however, heavily based on a constant density case. Still, in section IA of [11], it is stated that the superfluid component of the DM halo should have an approximately homogeneous density, and from the condensate profile displayed in figure 6.1, this seems to be the case. This justifies the use of the expressions in chapter 3. Based on an expression from the same chapter, the mass/energy of a single vortex, using the particle DM mass of $m_{\text {center }} \sim 10^{-4} \mathrm{eV}$, was estimated to approximately $10^{-4} \mathrm{~kg}$. This is an immense upgrade from the $10^{-14} \mathrm{~kg}$ result of section 5.2. The change is brought on by the change in particle mass, but also from the change in the density profile. Still, we found that combining the mass of all the vortices roughly amounted to the mass of Europa, one of Jupiter's moons. Using the actual dark matter density profile along

\footnotetext{
${ }^{2}$ For clarification, $\Lambda$ is a part of the Lagrangian formalism, while $\sigma / m$ is a particle property.
} 
with a more realistic distribution of the vortices might lead to a different result. However, the scales we are working with here strongly indicate that the vortices will be negligible in the big picture.

3. In our parameter variation process we also assessed the $\alpha$ parameter of the model, which had previously not been used in the calculations. It occurs in the phononbaryon interaction term of the Lagrangian density of the model, which is the term responsible for the appearance of a MONDian force. However, the $\alpha$ parameter appears together with the $\Lambda$ parameter, and their values are completely degenerate. This means that an increase in one parameter leads to a decrease in the other, keeping their relation constant to reproduce the MOND critical acceleration. The $\alpha$ parameter therefore had no impact on our parameter variation.

4. A large portion of [11] is devoted to calculating a more accurate superfluid DM density profile, taking into account finite-temperature effects, and using this along with observational data of baryonic density profiles to calculate rotation curves for two specific galaxies. This results in rotation curves that match the data well. A question is then whether or not our parameter variation would be able to reproduce realistic rotation curves. We have been able to change $m$ and adjust $\Lambda$ accordingly to reproduce the original zero-temperature condensate density profile, and it therefore seemed plausible that all our new parameter combinations should produce similar rotation curves. To test this out, we followed the method outlined in [11], however, with simplified toy density profiles for both the dark matter and baryonic matter. As our goal was to test whether or not our new parameter combinations produced similar rotation curves, and not to reproduce specific observations, this approach was sufficient. As can be seen in figure 6.7, the different parameter combinations do produce similar rotation curves, as suspected.

It might seem obvious that as long as we have altered $m$ and $\Lambda$ to reproduce the same density profile, all the different variations of the parameter values should produce the same rotation curves. However, the values of $m$ and $\Lambda$ are weighted differently in various equations, and one could therefore not be completely sure of reproduction. We may also observe in the aforementioned figure that the curves are not completely identical. This might be the effect of them being weighted differently in the equations, or a result of inaccuracy in our matching process (finding $\Lambda$ ). In any case, it seems like altering the value of $m$ and $\Lambda$ should reproduce rotation curves just fine.

We would like to point out that the result of this investigation is valuable in itself. Initially, we were motivated to study the rotation curves to make sure that altering the parameters of the model still gave the same promising results as the original parameters. This was again a consequence of looking for parameters that would give larger or more "massive" vortices. Now, the results of our rotation curve study tell us that observing rotation curves from various galaxies may not tell us the parameters of the superfluid DM model, as there seems to be a vast number of parameter combinations that would give the same rotation curves. However, in [11] it is discovered that the superfluid DM model does predict a slight rise in the rotation 
curve at large radii for high-surface-brightness (HSB) galaxies. As illustrated in their figure 6, when keeping the mass of the superfluid DM particle constant, the slope changes for different values of $\Lambda$. Observing a rise in the curve at large radii could therefore be an observational way of distinguishing the superfluid DM model from other models, and the precise slope might also help determine some ratio between $m$ and $\Lambda$. After all, the value of $\Lambda$ is not directly decided by the value of $m$, only constricted by the lower limit $\Lambda m^{3} \gtrsim 0.08 \mathrm{meV} \times \mathrm{eV}^{3}$.

5. Why Berezhiani and Khoury have focused on $\mathrm{eV}$ and meV scales is not quite clear at the moment. So far, there seems to be plenty of variables that would fulfill the various constraints put up by them in their papers. In addition, a more commonly expected axion mass lies in the $10^{-5}-10^{-3} \mathrm{eV}$ range, making the $\mathrm{eV}$ scales assumed by them quite large. Based on this, we also tested out which $\Lambda$-values would be necessary to reproduce the original density profile if we looked at some smaller masses, all the way down to $10^{-15} \mathrm{eV}$. However, this soon led us to $\Lambda$-values on the Planck mass scale and beyond, indicating the need of quantum gravity considerations. We therefore ended the parameter variations without reaching the $10^{-22} \mathrm{eV}$ mass of the fuzzy dark matter particles. The lowest possible mass, if we consider reaching Planck mass scales as an upper limit of $\Lambda$, seems to be around $\sim 10^{-10} \mathrm{eV}$. This would result in vortices of $\sim 1000 \mathrm{~km}$ size at the center of the condensate, and $\sim 18000 \mathrm{~km}$ at the edge. This DM particle mass would give a vortex "mass" of roughly $10^{8} \mathrm{~kg}$, significantly larger than the one meter vortex core case. Still, adding all the vortices together, this does not amount to more than ten thousand Sun-like stars. Therefore, even at such small particle DM masses, and $\Lambda$-values close to the Planck mass scale, the vortices seem to be of little significance in the galaxy as a whole. It might be the case that a full grid of such vortices could have additional affects, and this should be further investigated in the future.

Since we are discussing the mass of the dark matter particle, it might also be worth mentioning that the proposed particle of this model only is assumed to be "axionlike." For this model, this means that nothing more specific has been decided other than the particles being lightweight and having a bosonic nature. This is also why we decided to test some of the more conventional axion masses, to see whether or not these were ruled out by some constraints. Still, based on our results, it seems like masses around $\sim 10^{-3}-10^{-5} \mathrm{eV}$ are very much up for discussion.

In summary, we know that the vortices are very small and that they are separated by vast amounts of space compared to their size. The parameter values chosen by Berezhiani and Khoury to fit all constraints based on observations may be strongly altered and still result in the same condensate profile and, most likely, the same rotation curves. This alteration may also result in vortices of larger "mass" and size, which might impact the surroundings to a larger extent, if we imagine some kind of dragging effect around the vortices. Also, even though the effect of one vortex might be small, there is a chance that a full grid could have a greater effect, which could possibly be detected through lensing in the future, as proposed in section 10 of [1]. Whether or not this 
is plausible might be calculated in advance, but would require a model of the full vortex grid, ideally estimated without rigid body rotation to obtain a more accurate distribution of vortices. However, the new calculations of the thermalization radius in [11] result in smaller superfluid cores, which means that most of the lensing signals would come from the NWF part of the galaxy density profile (bottom of section III in [11]), where there are no vortices. This makes any observational detections unlikely, unless the large grid of vortices has a significant impact on, for example, the overall rotational behavior of the baryons far away from the vortices.

\subsection{Chapter 7 - Vortex-Baryon Interaction}

In chapter 7 we turned the table on our original goal of investigating how the vortices impact the baryons and instead had a look at how the baryons affect a single vortex. This was done by adding an interaction term to the simple Lagrangian density assessed in section 4.3. The term that was added was a current-current interaction term on the form $\mathscr{L}_{I}=f j_{S F}^{\mu} j_{\mu b}$. As stated in [72], this is the only Lorentz invariant interaction term in Minkowski space, and thus highly motivated. This leads to Euler-Lagrange equations that differ from the ones obtained in section 4.3 by an additional term. There is not enough information to solve the equations and find the new vortex profile, but it seems clear that the baryons will affect the vortex in some way or another.

To exactly find the impact of the interaction term, we need further information about the baryonic velocity field and density. This may be obtained from a more complex Lagrangian density, which incorporates the full dynamics of the system. We made a few attempts at adding kinetic energy terms and varying the interaction term in hopes of obtaining equations that could explain the system more fully. The different combinations we experimented with are shown in table 9.1.

Table 9.1: Overview of different interaction and kinetic terms added to the superfluid Lagrangian in various combinations. The first entry is the version presented in chapter 7. The others do not lead to consistent equations of motion, or any additional information beyond the first. The two currents are given as $j_{S F}^{\mu}=\rho_{S F}^{2} \partial^{\mu} \psi$ and $j_{b}^{\mu}=\rho_{b} v_{b}$.

\begin{tabular}{cc}
\hline \hline Interaction term & Kinetic term \\
\hline$f j_{S F}^{\mu} j_{\mu b}$ & 0 \\
$f j_{S F}^{\mu} j_{\mu b}$ & $\frac{1}{2} \rho_{b} v_{b}^{2}$ \\
$f \rho_{S F} v_{S F} \rho_{b} v_{b}$ & $\frac{1}{2} \rho_{b} v_{b}^{2}$ \\
$-\alpha \frac{\Lambda}{M_{\mathrm{Pl}}} \psi \rho_{b}$ & 0 \\
$-\alpha \frac{\Lambda}{M_{\mathrm{Pl}}} v_{S F} \rho_{b}$ & 0 \\
\hline
\end{tabular}


None of the combinations in table 9.1 contributed to additional information, and the ones including a kinetic term mostly gave inconsistent equations. This is probably because the kinetic term is incomplete and not Lorentz invariant in its given form. The addition of these terms was only attempted to guide us in the correct direction in order to construct a more elaborate Lagrangian, describing the whole system. The two last terms in the table were attempted as they are on the same form as the phononbaryon interaction of the superfluid DM model (see section 6.3). It is also mentioned, in section 1.3 of [1], that the form of the given interaction term could result from some sort of vortex interaction. We tried investigating this statement further, but none of the mentioned sources seem to explain exactly what is claimed in the aforementioned paper. However, we decided to give it a go, although with no luck. We therefore decided to go with the current-current interaction, as this is well motivated. In addition, we chose to ignore any kinetic energy term from the baryons, as we are studying the behavior of the dark matter. As there is a lot more dark matter than baryonic matter, this should be fine as a first approach.

One of the goals of this thesis was to investigate the impact that the superfluid DM vortices could have on the surrounding baryons. Upon discovering that the vortices have a very low energy, and probably a negligible effect on the baryons, an interesting question emerged: could the baryons affect the vortices instead? This chapter tells us that the baryons most likely will have an effect on the vortices, although a more extensive Lagrangian must be investigated in order to understand the full extent of the impact.

\subsection{Chapter 8 - Superfluid Dark Matter Lagrangian}

In chapter 8 we considered the relativistic completion of the model presented in [1]. Our goal was to see if we could extract a vortex equation for the given theory and check if it had a vortex solution similar to the one presented in figure 4.3. This was done by finding the related Euler-Lagrange equation of the full relativistic Lagrangian density, with respect to the parameter $\rho_{S F}$, assuming the same qualities as the regular vortex equation obtained in section 4.3.1. This resulted in a second-order nonlinear ordinary differential equation (ODE) with associated boundary conditions.

Our first approach was to try to solve the equation directly. As shown in the beginning of section 8.3.4, if we insert the expected boundary condition far from the center $(\eta \rightarrow \infty, R \rightarrow 1$ and $\dot{R} \rightarrow 0$ ), we do obtain $\ddot{R}=0$, which at least indicates that the equation is self-consistent. A lot of research went into the process of finding a method that could solve our boundary value problem (BVP), and most of the methods for solving second-order nonlinear ODEs as BVPs proved difficult and hard to implement.

One such method is the shooting method. Here, one makes a guess at an initial value, integrates the equation and checks whether or not the solution has the correct value at the boundary. If not, a new guess at the initial value is performed, and the equation is solved again. From our analysis in section 8.3.4, it is clear that we have not been able to solve our equation using the aforementioned method. In addition to 
making a guess at the initial value of $\dot{R}$, we also experimented with different values of $\Lambda_{c}$, although with an upper limit, $\Lambda_{c} \approx 0.63 \Lambda$, from observational constraints. If this upper limit had not existed, obtaining a solution of equation (8.37) with a similar shape as the original vortex solution would seem more likely. It would, however, if at all possible, require a fine-tuning of the initial condition of $\dot{R}$ and the value of $\Lambda_{c}$. As we have an upper limit of $\Lambda_{c}$, the region with the most promising parameter combinations is excluded from the analysis. From this, it therefore seems quite unlikely that we can find a promising vortex solution of equation (8.37). We do obtain a few good parameter combinations in our analysis, but taking a closer look at the actual solution for the given parameters we find a profile that does not fit very well with our boundary conditions, and also overflows at $\eta \sim 4$. It should be mentioned that we have not allowed $m$ and $\Lambda$ to change in our analysis, and it could very well be that letting them do so would result in a larger region in parameter space $\left(m, \Lambda, \Lambda_{c}\right.$ and $\left.\dot{R}_{\text {init }}\right)$ where a solution could be found. It might also be the case that the shooting method is too unstable for our purpose, and that other, more advanced and specialized methods should be applied.

In addition to the shooting method, we also tried applying Newton's method and the solve_bvp module from SciPy [58]. The latter was inspired by the success in solving equation (4.36), but did not work for the more advanced equation (8.37). The solve_bvp algorithm depends heavily on the initial guess, which means that we should already be pretty confident in the behavior of the solution before applying it. More details about the shooting method, Newton's method and the solve_bvp module may be found in appendix B.

Now, at this point we have at least five possible alternatives:

1. There exists a vortex solution similar to the one obtained from equation (4.36).

2. There exists a vortex solution different from the one obtained from equation (4.36).

3. There does not exist a vortex solution.

4. The analytical work must be reconsidered.

5. The Lagrangian density must be reconsidered.

From our analysis in section 8.3.4, we can not, with absolute certainty, rule out any of the first three alternatives. We have not been able to obtain a vortex solution yet, neither similar nor different from the one obtained from equation (4.36). In figure 8.4 it is clear that most $\dot{R}_{\text {init }}$ and $\Lambda_{c}$ combinations result in solutions that quickly diverge or fail to fulfill the boundary conditions. However, there is also a very small area where the parameter combinations result in solutions that the code regards as "good." From figure 8.5, we see that this area in parameter space is unstable, and that only slight changes to the parameters will result in solutions that are regarded as much "worse." By taking a closer look at the solutions of the "good" parameter combinations, we find that the vortex profiles do not look like anything we would expect, and still diverges for larger values of $\eta$. Based on this, point three seems more likely than points one and 
two at the present time. It could also be the case, as mentioned earlier, that applying a different, more stable method would give a more definite solution.

When presenting the relativistic completion of the superfluid dark matter theory in [1], Berezhiani and Khoury made sure that the Lagrangian could reproduce the scalar MOND-action in a given limit. This does not necessarily mean that they made sure that the theory allows for a vortex solution. Based on this, it is not certain whether or not our attempt at finding a vortex solution could have had a positive result. Still, as the theory presented in section 6 of [1] only was one of several possible Lagrangian densities to describe the model, it might be more desirable to construct a theory where a vortex solution is simple to extract. It should be clear that not obtaining a solution to this exact equation does not indicate that the vortices are not there. If the dark matter is a superfluid and the dark matter halo rotates above the critical angular velocity, the vortices should be there.

Now, let us consider point four. The vortex equation has been extracted from the full relativistic Lagrangian density by analytical work (see appendix A). This means that it will always be a possibility that some mistake has occurred along the way. This could be regarding the calculations themselves, or it could concern the assumptions made to obtain the equation. Of course, we have made a huge effort to weed out any possible mistakes, but one can never be too certain.

Point five touches upon the need to alter the proposed theory. This was also mentioned earlier, as the vortex solution is difficult to extract, and it would be desirable to have vortices arise quite effortlessly from the model. There is also the fact that the Lagrangian,

$$
\mathscr{L}=-\frac{1}{2}\left(\left|\partial_{\mu} \phi\right|^{2}+m^{2}|\phi|^{2}\right)-\frac{\Lambda^{4}}{6\left(\Lambda_{c}^{2}+|\phi|^{2}\right)^{6}}\left(\left|\partial_{\mu} \phi\right|^{2}+m^{2}|\phi|^{2}\right)^{3},
$$

was constructed to reproduce the MOND scalar action, $\mathscr{L} \sim \Lambda X \sqrt{|X|}$, in the nonrelativistic limit and MOND regime $\Lambda_{c} \ll|\phi|$. Now, if we compare the aforementioned MOND scalar action to the first term in equation (6.1), namely $\frac{2 \Lambda\left(2 m^{3 / 2}\right)}{3} X \sqrt{|X-\beta Y|}$, we find that the one the Lagrangian density was made to reproduce is the zero temperature case. This means that the case we have studied does not describe the superfluid at some finite temperature, but rather at $T=0$, which is a simplification of reality. Now, having a more complex theory for the model that includes finite temperature effects could alter the results and bring about a "friendlier" vortex equation. Looking at equation (9.1), we should also point out that the MOND regime is given as $\Lambda_{c} \ll|\phi|$, while a vortex solution should be expected for small values of $|\phi|$. This means that a vortex would be outside of the MOND regime.

All in all, we have not, at the present time, found any plausible vortex solution for the theory presented in [1]. It might still be possible that a solution exists, however, for very specific parameter values. If this is the case, demanding a vortex solution for a given superfluid dark matter theory could help put some additional constraints on the parameters of the model. 


\section{Chapter 10}

\section{Conclusions}

"Am I thinking what I think I'm thinking?"

- The Doctor, Doctor Who: The Vampires of Venice.

In chapter 1 we presented the motivation behind our work as "The goal of this thesis us thus to investigate the properties and possible impact of vortices on the dynamics of spiral galaxies. This will first be done by using a simplified model, which has the bonus of allowing us to analyze the parameter space left largely unexplored by the authors of [1]. We will then take steps to extend the investigation to the full, relativistic Lagrangian of the model." In chapters 5, 6, 7 and 8 we presented the methods and results of our research, and in chapter 9 we discussed them. It is now time to draw some final conclusions and set the stage for future investigations.

In this thesis, we have worked with a new dark matter theory, introduced by Berezhiani and Khoury in [1]. The dark matter is presented as a superfluid, which has collective excitations known as phonons. These interact with baryons, and in the process mediate a MONDian force. From this, the model is able to combine MOND with the $\Lambda$ CDM theory, ensuring results that fit observations well, both on galactic and cosmic scales. For the superfluid dark matter to have the desired properties, an equation of state $P \sim \rho^{3}$ is required. This is a result of letting the MOND scalar action describe the phonons, necessary to obtain the MONDian properties on galactic scales. Similar dark matter theories have been proposed before, although with a Bose-Einstein condensate dark matter, and not a superfluid [77, 80, 81]. These models have had $P \sim \rho^{2}$, meaning no MONDian force mediated by the phonons ${ }^{1}$.

By considering a constant density DM halo, we find that a galaxy rotates fast enough that a vortex grid should be present in the superfluid part of the DM halo. Assuming uniform rotation, the vortices are separated by a distance of $0.002 \mathrm{AU}$, and have a core size of $1 \mathrm{~mm}$. The latter coincides with the simple estimate presented in section 10 of [1]. We also calculate the energy and corresponding "mass" of a vortex and find that

\footnotetext{
${ }^{1}$ In general, $P \sim \rho^{2}$ means that two-body interactions dominate in the gas/fluid, while $P \sim \rho^{3}$ tells us that three-body interactions dominate. For our superfluid DM, we must therefore have that the two-body interactions of the DM particles are negligible compared to the three-body interactions.
} 
it is incredibly small. The mass of a single vortex, located at the rotational axis of the galaxy, is roughly $10^{-14} \mathrm{~kg}$. This indicates that any impact on the surroundings is unlikely.

Moving on to study a condensate density profile as presented in section 3.2 of [1], we find that the vortex core size varies throughout the condensate. For the parameters $m \sim \mathrm{eV}$ and $\Lambda \sim \mathrm{meV}$, this indicates that the vortex core is roughly ten times smaller in the center than at the edge. As one of our goals was to investigate the impact of the vortices, the current situation made us wonder if there were any changes that could be made in order to make the vortices more important in the big picture. We discovered that a reduction in the dark matter particle mass, along with an increase in the value of $\Lambda$, would result in vortices with a larger energy and "mass." Changing the parameters also seems to uphold all model constraints as presented in [11], along with reproducing the same rotation curves as for the original parameters. In studying the rotation curves we also find, as an independent result, that observing rotation curves from different galaxies can not necessarily tell us anything about the parameter values of the model. Why Berezhiani and Khoury originally focused on masses of $\sim \mathrm{eV}$ scale is not certain, although some other BEC DM models have proposed similar mass scales [80]. Some other models, however, propose masses of $\sim 10^{-22} \mathrm{eV}[81,82]$, which would result in larger vortex cores and a considerable "mass" per vortex in the superfluid DM model. Still, when experimenting with low masses, we find that masses smaller than $\sim 10^{-10} \mathrm{eV}$ result in $\Lambda$-values above the Planck mass scale. For masses $\sim 10^{-15} \mathrm{eV}$, the vortex cores also become so big that they start overlapping. A BEC could remain stable in this case, but the vortices could no longer be treated individually, and the grid would have to be assessed differently. For the particle DM mass of $m=10^{-10} \mathrm{eV}$, we find that the total "mass" contribution from all the vortices in the superfluid component is $\sim 10000 M_{\odot}$. It is therefore deemed unlikely that the vortices would affect the movement of the baryons in any significant way.

From the superfluid DM theory presented in section 6 of [1], we extract a vortex equation by assuming that the modulus of the scalar field describing the dark matter only has a radial dependence, and that the corresponding phase only depends upon the polar coordinate angle, $\theta$. These are the same assumptions that were made in order to reproduce the known vortex equation of section 4.3.1. As of now, we have not found a solution of the superfluid DM vortex equation that is similar to known vortex profiles, or at all realistic. It is unsure whether or not the given theory has a vortex solution, but one could possibly be found for extremely fine-tuned values of the model parameter $\Lambda_{c}$ and initial conditions. However, observational constraints rule out the most promising values of $\Lambda_{c}$, and any realistic vortex solution of equation (8.37) therefore seems unlikely. Based on this, it would be desirable to produce a different Lagrangian density that gives the MOND scalar action in certain limits, and a vortex profile that is easier to extract and does not require a fine-tuning of parameters. In addition, this new Lagrangian density should include finite-temperature effects. That being said, our vortex-baryon interaction investigation tells us that the vortex profile most likely will be altered as a result of interactions between the vortices and baryons. This means that we must expect a superfluid DM vortex profile that differs, at least slightly, from the single 
straight-line vortex profile presented in section 4.3.1. In addition, even though we have not been able to find a solution at the current time, we can not require that the vortex profile behaves as we want it to, or that the situation is at all simple. The Universe may behave as the Universe wants to behave.

\subsection{Future Work}

Throughout the thesis there have been many mentions of future work. Here, at the very end, we would like to summarize the main ones and lay a possible path for future research.

Firstly, it would be interesting to calculate or simulate the full vortex grid. This is because, as mentioned before, the distribution does not have to be uniform. As shown in [61], a condensate that does not rotate uniformly will produce a vortex grid that has a ring formation. If this is the case for our superfluid DM component, it could have a different impact on the surroundings. However, understanding how the dark matter halo rotates is quite a challenge [83], and assuming different kinds of rotation and calculating/simulating the corresponding vortex grid might be the best solution at the present time. Still, there is one big problem. For the DM particle mass scales presented in $[1,11]$, the vortices are incredibly small and well below any current galaxy simulation resolution [84]. Simulating the vortices and their impact on the galaxy would be the ultimate goal, but with the current technology it seems difficult enough to deal with the vortex grid alone. This should therefore not be considered future work, but rather far into the future work ${ }^{2}$.

Secondly, as the results of chapter 6 seem to allow for parameter changes in the superfluid DM model, the vortex equation should be analyzed in the full parameter space of $m, \Lambda, \Lambda_{c}$ and $\dot{R}_{\text {init }}$. As of now, we found no solution when experimenting with $\Lambda_{c}$ and $\dot{R}_{\text {init }}$, but it could be the case that different values of $m$ and $\Lambda$ would allow for a more stable solution. In addition, one could try to specialize a solving algorithm to fit our specific vortex equation. It could also be interesting to investigate a possible rewrite of the equation to make the terms more manageable. We should also keep in mind that the vortex-baryon interaction assessment suggests that the vortex profile will be different from the one obtained in section 4.3.1, although this should also be investigated further using a more extensive Lagrangian density.

As a third remark, the rotation curve assessment of section 6.4 should also be performed with full dark matter density profiles and baryonic profiles obtained from observations. This was not done in this thesis, as it required some additional numerical work, which was not necessary in order to obtain the needed information. Still, for completeness, this should be considered in the future.

In [1], lensing was proposed as a possible way to observe the effect of vortices. In [11], however, new density profiles were calculated, which led to a smaller part of the dark matter halo being in the superfluid component. This means that most of the gravitational lensing signal would come from the non-condensed part of the DM

\footnotetext{
${ }^{2}$ Although, we could get lucky and experience som technological revolution on the resolution front.
} 
halo, which has no vortices. This means that, although we know that lensing does not challenge the idea of a superfluid dark matter model [85], it can probably not be used to detect the presence of vortices. However, if the effect of vortices were to be detected somehow, it could help us constrain the parameters of the model. As we have seen, the mass of the DM particle greatly affects the "mass" of a single vortex. Thus, detecting any extra gravitational effects on baryons, consistent with the presence of a grid of some kind, could help us decide the DM particle mass, whether this detection be through gravitational lensing or by other means.

It seems more probable that it is other observable effects that will help distinguish the superfluid dark matter model from other models. For example, in section VII B of [11], it is discovered that the model predicts a small increase in the rotation curves at large distances for high-surface-brightness (HSB) galaxies. Measuring this increase could help distinguish the given model from others. In addition, there is a transition region between the thermalization radius, within which the dark matter can condense, and the NFW radius, outside which the DM density profile may be fitted to a NFW profile. Calculating the profile between the two radii was also left to future work by the authors in [11]. Based on this, the transition region could leave some characteristic features in the rotation curves of galaxies. Once this is calculated, it could be used as an observational constraint of the model. In a paper by Cai, Liu and Wang [86], gravitational waves are also proposed as a way of exploring the parameter space of the superfluid dark matter model. This investigation could be interesting to keep an eye on in the future.

In conversation with Benoit Famaey [79], it was mentioned that the authors of [11] are working on a new model to unite MOND and $\Lambda$ CDM. It could therefore be advisable to wait for this new assessment, or reach out to the authors, in advance of further investigations of the superfluid dark matter model. Still, the future of the given model is exciting, and there has even been proposed a unified dark sector in the superfluid DM picture [87]. Uniting MOND and the $\Lambda$ CDM theory is very intriguing from an observational perspective. The astonishing similarities between MOND predictions and galaxy observations are hard to ignore, and finally fitting this surprisingly simple idea from 1983 into the bigger picture would be a considerable step in the direction of understanding the behavior of the Universe we live in. 
Appendices 



\section{Appendix A}

\section{Relativistic Lagrangian Calculations}

This appendix is a copy of section 8.2, presenting detailed calculations showing every step on the way to obtaining the vortex equation for the Lagrangian presented in section 6 of [1].

We now want to extract an equation of motion describing the vortices in the superfluid DM model. The Lagrangian is given by:

$$
\mathscr{L}=-\frac{1}{2}\left(\left|\partial_{\mu} \phi\right|^{2}+m^{2}|\phi|^{2}\right)-\frac{\Lambda^{4}}{6\left(\Lambda_{c}^{2}+|\phi|^{2}\right)^{6}}\left(\left|\partial_{\mu} \phi\right|^{2}+m^{2}|\phi|^{2}\right)^{3},
$$

where $\Lambda_{c}$ is an energy scale introduced to allow $\phi=0$. As before, we will use the expression

$$
\phi=\frac{\rho(X)}{\sqrt{2}} e^{i \psi(X)}
$$

for the wavefunction, assume a static solution so that $\partial_{0} \rho=0$, and use $\partial_{0} \psi=\mu$. We will omit the label $S F$, as it is clear in this context that it is the superfluid properties that are being calculated. This gives the term

$$
\begin{aligned}
\left|\partial_{\mu} \phi\right|^{2} & =\partial_{\mu} \phi \partial^{\mu} \phi^{*} \\
& =g^{\mu \nu} \partial_{\mu} \phi \partial_{\nu} \phi^{*} \\
& =g^{\mu \nu} \partial_{\mu}\left(\frac{1}{\sqrt{2}} \rho e^{i \psi}\right) \partial_{\nu}\left(\frac{1}{\sqrt{2}} \rho e^{-i \psi}\right) \\
& =\frac{1}{2} g^{\mu \nu}\left(e^{i \psi} \partial_{\mu} \rho+\rho \partial_{\mu} e^{i \psi}\right)\left(e^{-i \psi} \partial_{\nu} \rho+\rho \partial_{\nu} e^{-i \psi}\right) \\
& =\frac{1}{2} g^{\mu \nu}\left(e^{i \psi} \partial_{\mu} \rho+i \rho e^{i \psi} \partial_{\mu} \psi\right)\left(e^{-i \psi} \partial_{\nu} \rho-i \rho e^{-i \psi} \partial_{\nu} \psi\right) \\
& =\frac{1}{2} g^{\mu \nu}\left(\partial_{\mu} \rho \partial_{\nu} \rho-i \rho \partial_{\mu} \rho \partial_{\nu} \psi+i \rho \partial_{\mu} \psi \partial_{\nu} \rho-i^{2} \rho^{2} \partial_{\mu} \psi \partial_{\nu} \psi\right) .
\end{aligned}
$$


As $\mu$ and $\nu$ are dummy indices in this case, they may be switched in one of the two middle terms, so that we end up with

$$
\left|\partial_{\mu} \phi\right|^{2}=\frac{1}{2} g^{\mu \nu} \partial_{\mu} \rho \partial_{\nu} \rho+\frac{1}{2} g^{\mu \nu} \rho^{2} \partial_{\mu} \psi \partial_{\nu} \psi
$$

to be inserted in equation (A.1). As we are looking for a vortex solution, we will use the Minkowski metric in polar coordinates, that is, $g_{\mu \nu}=\operatorname{diag}\left(-1,1, \mathrm{r}^{2}, 1\right)$. The Lagrangian now has the form

$$
\begin{aligned}
\mathscr{L}= & -\frac{1}{2}\left(\frac{1}{2} g^{\mu \nu} \partial_{\mu} \rho \partial_{\nu} \rho+\frac{1}{2} g^{\mu \nu} \rho^{2} \partial_{\mu} \psi \partial_{\nu} \psi+\frac{1}{2} m^{2} \rho^{2}\right) \\
& -\frac{\Lambda^{4}}{6\left(\Lambda_{c}^{2}+\frac{1}{2} \rho^{2}\right)^{6}}\left(\frac{1}{2} g^{\mu \nu} \partial_{\mu} \rho \partial_{\nu} \rho+\frac{1}{2} g^{\mu \nu} \rho^{2} \partial_{\mu} \psi \partial_{\nu} \psi+\frac{1}{2} m^{2} \rho^{2}\right)^{3} \\
= & -\frac{1}{4}\left(g^{\mu \nu} \partial_{\mu} \rho \partial_{\nu} \rho+g^{\mu \nu} \rho^{2} \partial_{\mu} \psi \partial_{\nu} \psi+m^{2} \rho^{2}\right) \\
& -\frac{\Lambda^{4}}{48\left(\Lambda_{c}^{2}+\frac{1}{2} \rho^{2}\right)^{6}}\left(g^{\mu \nu} \partial_{\mu} \rho \partial_{\nu} \rho+g^{\mu \nu} \rho^{2} \partial_{\mu} \psi \partial_{\nu} \psi+m^{2} \rho^{2}\right)^{3} .
\end{aligned}
$$

To properly keep track of all the indices, we write out the quadratic term as follows:

$$
\begin{aligned}
\mathscr{L}=-\frac{1}{4}\left(g^{\mu \nu} \partial_{\mu} \rho \partial_{\nu} \rho\right. & \left.+g^{\mu \nu} \rho^{2} \partial_{\mu} \psi \partial_{\nu} \psi+m^{2} \rho^{2}\right) \\
-\frac{\Lambda^{4}}{48\left(\Lambda_{c}^{2}+\frac{1}{2} \rho^{2}\right)^{6}} & \left(g^{\mu \nu} \partial_{\mu} \rho \partial_{\nu} \rho+g^{\mu \nu} \rho^{2} \partial_{\mu} \psi \partial_{\nu} \psi+m^{2} \rho^{2}\right) \\
\times & \left(g^{\alpha \beta} \partial_{\alpha} \rho \partial_{\beta} \rho+g^{\alpha \beta} \rho^{2} \partial_{\alpha} \psi \partial_{\beta} \psi+m^{2} \rho^{2}\right) \\
\times & \left(g^{\omega \sigma} \partial_{\omega} \rho \partial_{\sigma} \rho+g^{\omega \sigma} \rho^{2} \partial_{\omega} \psi \partial_{\sigma} \psi+m^{2} \rho^{2}\right),
\end{aligned}
$$

and to make the calculations more structured we will assign the names

$$
\begin{aligned}
\mathscr{L}_{m n} & =g^{\mu \nu} \partial_{\mu} \rho \partial_{\nu} \rho+g^{\mu \nu} \rho^{2} \partial_{\mu} \psi \partial_{\nu} \psi+m^{2} \rho^{2}, \\
\mathscr{L}_{a b} & =g^{\alpha \beta} \partial_{\alpha} \rho \partial_{\beta} \rho+g^{\alpha \beta} \rho^{2} \partial_{\alpha} \psi \partial_{\beta} \psi+m^{2} \rho^{2}, \\
\mathscr{L}_{o s} & =g^{\omega \sigma} \partial_{\omega} \rho \partial_{\sigma} \rho+g^{\omega \sigma} \rho^{2} \partial_{\omega} \psi \partial_{\sigma} \psi+m^{2} \rho^{2} .
\end{aligned}
$$

In section 4.3.1, we obtained a vortex equation by finding the Euler-Lagrange equation with respect to $\rho$ :

$$
\frac{\partial \mathscr{L}}{\partial \rho}-\partial_{\eta} \frac{\partial \mathscr{L}}{\partial\left(\partial_{\eta} \rho\right)}=0
$$

We will take the same approach here. Let us start with the easiest part and look at 
equation (A.12) to find

$$
\begin{aligned}
\frac{\partial \mathscr{L}}{\partial \rho}= & -\frac{1}{4}\left(2 g^{\mu \nu} \rho \partial_{\mu} \psi \partial_{\nu} \psi+2 m^{2} \rho\right) \\
& +6 \frac{\Lambda^{4}}{48\left(\Lambda_{c}^{2}+\frac{1}{2} \rho^{2}\right)^{7}} \rho \mathscr{L}_{m n} \mathscr{L}_{a b} \mathscr{L}_{o s} \\
& -\frac{\Lambda^{4}}{48\left(\Lambda_{c}^{2}+\frac{1}{2} \rho^{2}\right)^{6}}\left(2 g^{\mu \nu} \rho \partial_{\mu} \psi \partial_{\nu} \psi+2 m^{2} \rho\right) \mathscr{L}_{a b} \mathscr{L}_{o s} \\
& -\frac{\Lambda^{4}}{48\left(\Lambda_{c}^{2}+\frac{1}{2} \rho^{2}\right)^{6}} \mathscr{L}_{m n}\left(2 g^{\alpha \beta} \rho \partial_{\alpha} \psi \partial_{\beta} \psi+2 m^{2} \rho\right) \mathscr{L}_{o s} \\
& -\frac{\Lambda^{4}}{48\left(\Lambda_{c}^{2}+\frac{1}{2} \rho^{2}\right)^{6}} \mathscr{L}_{m n} \mathscr{L}_{a b}\left(2 g^{\omega \sigma} \rho \partial_{\omega} \psi \partial_{\sigma} \psi+2 m^{2} \rho\right) \\
= & -\frac{1}{2}\left(g^{\mu \nu} \rho \partial_{\mu} \psi \partial_{\nu} \psi+m^{2} \rho\right) \\
& +\frac{\Lambda^{4} \rho}{8\left(\Lambda_{c}^{2}+\frac{1}{2} \rho^{2}\right)^{7}}\left(g^{\mu \nu} \partial_{\mu} \rho \partial_{\nu} \rho+g^{\mu \nu} \rho^{2} \partial_{\mu} \psi \partial_{\nu} \psi+m^{2} \rho^{2}\right)^{3} \\
& -\frac{\Lambda^{4}}{8\left(\Lambda_{c}^{2}+\frac{1}{2} \rho^{2}\right)^{6}}\left(g^{\mu \nu} \rho \partial_{\mu} \psi \partial_{\nu} \psi+m^{2} \rho\right)\left(g^{\alpha \beta} \partial_{\alpha} \rho \partial_{\beta} \rho+g^{\alpha \beta} \rho^{2} \partial_{\alpha} \psi \partial_{\beta} \psi+m^{2} \rho^{2}\right)^{2} .
\end{aligned}
$$

As $\mu, \nu, \alpha, \beta, \omega$ and $\sigma$ are dummy indices we have that $\mathscr{L}_{m n}=\mathscr{L}_{a b}=\mathscr{L}_{o s}$, which is the reason for the transition between equation (A.17) and (A.18). The use of $\mathscr{L}_{m n}$, $\mathscr{L}_{a b}$ and $\mathscr{L}_{o s}$ was not important for this particular calculation, but is used to make sure that everything is done correctly in the next step where we use equation (A.12) to obtain

$$
\begin{aligned}
\frac{\partial \mathscr{L}}{\partial\left(\partial_{\eta} \rho\right)}= & -\frac{1}{4}\left(g^{\mu \nu} \frac{\partial\left(\partial_{\mu} \rho\right)}{\partial\left(\partial_{\eta} \rho\right)} \partial_{\nu} \rho+g^{\mu \nu} \partial_{\mu} \rho \frac{\partial\left(\partial_{\nu} \rho\right)}{\partial\left(\partial_{\eta} \rho\right)}\right) \\
& -\frac{\Lambda^{4}}{48\left(\Lambda_{c}^{2}+\frac{1}{2} \rho^{2}\right)^{6}}\left(g^{\mu \nu} \frac{\partial\left(\partial_{\mu} \rho\right)}{\partial\left(\partial_{\eta} \rho\right)} \partial_{\nu} \rho+g^{\mu \nu} \partial_{\mu} \rho \frac{\partial\left(\partial_{\nu} \rho\right)}{\partial\left(\partial_{\eta} \rho\right)}\right) \mathscr{L}_{a b} \mathscr{L}_{o s} \\
& -\frac{\Lambda^{4}}{48\left(\Lambda_{c}^{2}+\frac{1}{2} \rho^{2}\right)^{6}} \mathscr{L}_{m n}\left(g^{\alpha \beta} \frac{\partial\left(\partial_{\alpha} \rho\right)}{\partial\left(\partial_{\eta} \rho\right)} \partial_{\beta} \rho+g^{\alpha \beta} \partial_{\alpha} \rho \frac{\partial\left(\partial_{\beta} \rho\right)}{\partial\left(\partial_{\eta} \rho\right)}\right) \mathscr{L}_{o s} \\
& -\frac{\Lambda^{4}}{48\left(\Lambda_{c}^{2}+\frac{1}{2} \rho^{2}\right)^{6}} \mathscr{L}_{m n} \mathscr{L}_{a b}\left(g^{\omega \sigma} \frac{\partial\left(\partial_{\omega} \rho\right)}{\partial\left(\partial_{\eta} \rho\right)} \partial_{\sigma} \rho+g^{\omega \sigma} \partial_{\omega} \rho \frac{\partial\left(\partial_{\sigma} \rho\right)}{\partial\left(\partial_{\eta} \rho\right)}\right) .
\end{aligned}
$$


Again $\mu, \nu, \alpha, \beta, \omega$ and $\sigma$ are dummy indices, and the three last terms are identical:

$$
\begin{aligned}
\frac{\partial \mathscr{L}}{\partial\left(\partial_{\eta} \rho\right)}= & -\frac{1}{4}\left(g^{\mu \nu} \delta_{\mu}^{\eta} \partial_{\nu} \rho+g^{\mu \nu} \partial_{\mu} \rho \delta_{\nu}^{\eta}\right) \\
& -\frac{3 \Lambda^{4}}{48\left(\Lambda_{c}^{2}+\frac{1}{2} \rho^{2}\right)^{6}}\left(g^{\mu \nu} \delta_{\mu}^{\eta} \partial_{\nu} \rho+g^{\mu \nu} \partial_{\mu} \rho \delta_{\nu}^{\eta}\right) \mathscr{L}_{a b} \mathscr{L}_{o s} .
\end{aligned}
$$

In the case of Minkowski space, $g_{\mu \nu}$ is symmetric, and as $\mu$ and $\nu$ again are dummy indices, we may simplify as

$$
\begin{aligned}
\frac{\partial \mathscr{L}}{\partial\left(\partial_{\eta} \rho\right)}=-\frac{1}{4}\left(g^{\eta \nu} \partial_{\nu} \rho\right. & \left.+g^{\mu \eta} \partial_{\mu} \rho\right)-\frac{\Lambda^{4}}{16\left(\Lambda_{c}^{2}+\frac{1}{2} \rho^{2}\right)^{6}}\left(g^{\eta \nu} \partial_{\nu} \rho+g^{\mu \eta} \partial_{\mu} \rho\right) \mathscr{L}_{a b} \mathscr{L}_{o s} \\
=-\frac{1}{2} g^{\eta \nu} \partial_{\nu} \rho & -\frac{\Lambda^{4}}{8\left(\Lambda_{c}^{2}+\frac{1}{2} \rho^{2}\right)^{6}} g^{\eta \nu} \partial_{\nu} \rho \\
\times & \left(g^{\alpha \beta} \partial_{\alpha} \rho \partial_{\beta} \rho+g^{\alpha \beta} \rho^{2} \partial_{\alpha} \psi \partial_{\beta} \psi+m^{2} \rho^{2}\right) \\
\times & \left(g^{\omega \sigma} \partial_{\omega} \rho \partial_{\sigma} \rho+g^{\omega \sigma} \rho^{2} \partial_{\omega} \psi \partial_{\sigma} \psi+m^{2} \rho^{2}\right) .
\end{aligned}
$$

The next step is then:

$$
\begin{aligned}
\partial_{\eta} \frac{\partial \mathscr{L}}{\partial\left(\partial_{\eta} \rho\right)}= & -\frac{1}{2}\left[\left(\partial_{\eta} g^{\eta \nu}\right) \partial_{\nu} \rho+g^{\eta \nu} \partial_{\eta}\left(\partial_{\nu} \rho\right)\right] \\
& -\partial_{\eta}\left(\frac{\Lambda^{4}}{8\left(\Lambda_{c}^{2}+\frac{1}{2} \rho^{2}\right)^{6}}\right) g^{\eta \nu} \partial_{\nu} \rho \mathscr{L}_{a b} \mathscr{L}_{o s} \\
& -\frac{\Lambda^{4}}{8\left(\Lambda_{c}^{2}+\frac{1}{2} \rho^{2}\right)^{6}}\left[\left(\partial_{\eta} g^{\eta \nu}\right) \partial_{\nu} \rho+g^{\eta \nu} \partial_{\eta}\left(\partial_{\nu} \rho\right)\right] \mathscr{L}_{a b} \mathscr{L}_{o s} \\
& -\frac{\Lambda^{4}}{8\left(\Lambda_{c}^{2}+\frac{1}{2} \rho^{2}\right)^{6}} g^{\eta \nu} \partial_{\nu} \rho\left[\left(\partial_{\eta} g^{\alpha \beta}\right) \partial_{\alpha} \rho \partial_{\beta} \rho+g^{\alpha \beta} \partial_{\eta}\left(\partial_{\alpha} \rho\right) \partial_{\beta} \rho+g^{\alpha \beta} \partial_{\alpha} \rho \partial_{\eta}\left(\partial_{\beta} \rho\right)\right. \\
& +\left(\partial_{\eta} g^{\alpha \beta}\right) \rho^{2} \partial_{\alpha} \psi \partial_{\beta} \psi+g^{\alpha \beta} \partial_{\eta}\left(\rho^{2}\right) \partial_{\alpha} \psi \partial_{\beta} \psi+g^{\alpha \beta} \rho^{2} \partial_{\eta}\left(\partial_{\alpha} \psi\right) \partial_{\beta} \psi \\
& \left.+g^{\alpha \beta} \rho^{2} \partial_{\alpha} \psi \partial_{\eta}\left(\partial_{\beta} \psi\right)+m^{2} \partial_{\eta}\left(\rho^{2}\right)\right] \mathscr{L}_{o s} \\
& -\frac{\Lambda^{4}}{8\left(\Lambda_{c}^{2}+\frac{1}{2} \rho^{2}\right)^{6}} g^{\eta \nu} \partial_{\nu} \rho \mathscr{L}_{a b}\left[\left(\partial_{\eta} g^{\omega \sigma}\right) \partial_{\omega} \rho \partial_{\sigma} \rho+g^{\omega \sigma} \partial_{\eta}\left(\partial_{\omega} \rho\right) \partial_{\sigma} \rho+g^{\omega \sigma} \partial_{\omega} \rho \partial_{\eta}\left(\partial_{\sigma} \rho\right)\right. \\
+ & +\left(\partial_{\eta} g^{\omega \sigma}\right) \rho^{2} \partial_{\omega} \psi \partial_{\sigma} \psi+g^{\omega \sigma} \partial_{\eta}\left(\rho^{2}\right) \partial_{\omega} \psi \partial_{\sigma} \psi+g^{\omega \sigma} \rho^{2} \partial_{\eta}\left(\partial_{\omega} \psi\right) \partial_{\sigma} \psi \\
& \left.+g^{\omega \sigma} \rho^{2} \partial_{\omega} \psi \partial_{\eta}\left(\partial_{\sigma} \psi\right)+m^{2} \partial_{\eta}\left(\rho^{2}\right)\right] .
\end{aligned}
$$


As before, $\alpha, \beta, \omega$ and $\sigma$ are dummy indices, and the two last terms are equal. In addition, $\mathscr{L}_{a b}=\mathscr{L}_{o s}$. Writing everything out we have

$$
\begin{aligned}
\partial_{\eta} \frac{\partial \mathscr{L}}{\partial\left(\partial_{\eta} \rho\right)}= & -\frac{1}{2}\left[\left(\partial_{\eta} g^{\eta \nu}\right) \partial_{\nu} \rho+g^{\eta \nu} \partial_{\eta}\left(\partial_{\nu} \rho\right)\right] \\
& +\frac{6 \Lambda^{4} \rho\left(\partial_{\eta} \rho\right)}{8\left(\Lambda_{c}^{2}+\frac{1}{2} \rho^{2}\right)^{7}} g^{\eta \nu} \partial_{\nu} \rho\left(g^{\alpha \beta} \partial_{\alpha} \rho \partial_{\beta} \rho+g^{\alpha \beta} \rho^{2} \partial_{\alpha} \psi \partial_{\beta} \psi+m^{2} \rho^{2}\right)^{2} \\
& -\frac{\Lambda^{4}}{8\left(\Lambda_{c}^{2}+\frac{1}{2} \rho^{2}\right)^{6}}\left[\left(\partial_{\eta} g^{\eta \nu}\right) \partial_{\nu} \rho+g^{\eta \nu} \partial_{\eta}\left(\partial_{\nu} \rho\right)\right] \\
& \times\left(g^{\alpha \beta} \partial_{\alpha} \rho \partial_{\beta} \rho+g^{\alpha \beta} \rho^{2} \partial_{\alpha} \psi \partial_{\beta} \psi+m^{2} \rho^{2}\right)^{2} \\
& -\frac{\Lambda^{4}}{4\left(\Lambda_{c}^{2}+\frac{1}{2} \rho^{2}\right)^{6}} g^{\eta \nu} \partial_{\nu} \rho\left[\left(\partial_{\eta} g^{\alpha \beta}\right) \partial_{\alpha} \rho \partial_{\beta} \rho+g^{\alpha \beta} \partial_{\eta}\left(\partial_{\alpha} \rho\right) \partial_{\beta} \rho+g^{\alpha \beta} \partial_{\alpha} \rho \partial_{\eta}\left(\partial_{\beta} \rho\right)\right. \\
& +\left(\partial_{\eta} g^{\alpha \beta}\right) \rho^{2} \partial_{\alpha} \psi \partial_{\beta} \psi+g^{\alpha \beta} \partial_{\eta}\left(\rho^{2}\right) \partial_{\alpha} \psi \partial_{\beta} \psi+g^{\alpha \beta} \rho^{2} \partial_{\eta}\left(\partial_{\alpha} \psi\right) \partial_{\beta} \psi \\
& \left.+g^{\alpha \beta} \rho^{2} \partial_{\alpha} \psi \partial_{\eta}\left(\partial_{\beta} \psi\right)+m^{2} \partial_{\eta}\left(\rho^{2}\right)\right] \\
& \times\left(g^{\omega \sigma} \partial_{\omega} \rho \partial_{\sigma} \rho+g^{\omega \sigma} \rho^{2} \partial_{\omega} \psi \partial_{\sigma} \psi+m^{2} \rho^{2}\right) .
\end{aligned}
$$

We want to check if we can extract a vortex solution from the Euler-Lagrange equations, and to do so we will insert what led to our previous vortex equation, mainly that $\rho_{S F}=\rho_{S F}(r), \psi=n \theta$ and $\partial_{0} \psi=\mu$ (see section 4.3.1). In addition, we know that our metric is diagonal, $g_{\mu \nu}=\operatorname{diag}\left(-1,1, \mathrm{r}^{2}, 1\right)$, and as we are using polar coordinates the indices cycle through $\mu=t, r, \theta, z$ (where $c=1$ ). We will also use the notation " "" to represent the derivative with respect to $r$. Inserting all of this into equation (A.18) results in

$$
\begin{aligned}
\frac{\partial \mathscr{L}}{\partial \rho}= & -\frac{1}{2}\left(g^{\mu \nu} \rho \partial_{\mu} \psi \partial_{\nu} \psi+m^{2} \rho\right) \\
& +\frac{\Lambda^{4} \rho}{8\left(\Lambda_{c}^{2}+\frac{1}{2} \rho^{2}\right)^{7}}\left(g^{\mu \nu} \partial_{\mu} \rho \partial_{\nu} \rho+g^{\mu \nu} \rho^{2} \partial_{\mu} \psi \partial_{\nu} \psi+m^{2} \rho^{2}\right)^{3} \\
& -\frac{\Lambda^{4}}{8\left(\Lambda_{c}^{2}+\frac{1}{2} \rho^{2}\right)^{6}}\left(g^{\mu \nu} \rho \partial_{\mu} \psi \partial_{\nu} \psi+m^{2} \rho\right)\left(g^{\alpha \beta} \partial_{\alpha} \rho \partial_{\beta} \rho+g^{\alpha \beta} \rho^{2} \partial_{\alpha} \psi \partial_{\beta} \psi+m^{2} \rho^{2}\right)^{2}
\end{aligned}
$$


120

Relativistic Lagrangian Calculations

$$
\begin{aligned}
= & -\frac{1}{2}\left(-\rho \mu^{2}+\rho \frac{n^{2}}{r^{2}}+m^{2} \rho\right) \\
& +\frac{\Lambda^{4} \rho}{8\left(\Lambda_{c}^{2}+\frac{1}{2} \rho^{2}\right)^{7}}\left(\dot{\rho}^{2}-\rho^{2} \mu^{2}+\rho^{2} \frac{n^{2}}{r^{2}}+m^{2} \rho^{2}\right)^{3} \\
& -\frac{\Lambda^{4}}{8\left(\Lambda_{c}^{2}+\frac{1}{2} \rho^{2}\right)^{6}}\left(-\rho \mu^{2}+\rho^{2} \frac{n^{2}}{r^{2}}+m^{2} \rho\right)\left(\dot{\rho}^{2}-\rho^{2} \mu^{2}+\rho^{2} \frac{n^{2}}{r^{2}}+m^{2} \rho^{2}\right)^{2} \\
= & -\frac{1}{2} q \rho+\frac{\Lambda^{4} \rho}{8\left(\Lambda_{c}^{2}+\frac{1}{2} \rho^{2}\right)^{7}}\left(\dot{\rho}^{2}+q \rho^{2}\right)^{3}-\frac{\Lambda^{4}}{8\left(\Lambda_{c}^{2}+\frac{1}{2} \rho^{2}\right)^{6}} q \rho\left(\dot{\rho}^{2}+q \rho^{2}\right)^{2},
\end{aligned}
$$

if we define

$$
q=m^{2}-\mu^{2}+\frac{n^{2}}{r^{2}} .
$$

We now want to do the same for equation (A.24). This equation is quite complicated, but we can do a few things to simplify. First of all, we know that $\rho=\rho(r)$, so the derivative with respect to anything but $r$ will be zero. Similarly, $\psi=\psi(\theta)$ and the derivative with respect to anything but $\theta$ will be zero. In addition, we know that the double derivative of $\psi$ is zero. This will save us some time and space. Let's go:

$$
\begin{aligned}
\partial_{\eta} \frac{\partial \mathscr{L}}{\partial\left(\partial_{\eta} \rho\right)}= & -\frac{1}{2}\left[\left(\partial_{\eta} g^{\eta \nu}\right) \partial_{\nu} \rho+g^{\eta \nu} \partial_{\eta}\left(\partial_{\nu} \rho\right)\right] \\
& +\frac{6 \Lambda^{4} \rho\left(\partial_{\eta} \rho\right)}{8\left(\Lambda_{c}^{2}+\frac{1}{2} \rho^{2}\right)^{7}} g^{\eta \nu} \partial_{\nu} \rho\left(g^{\alpha \beta} \partial_{\alpha} \rho \partial_{\beta} \rho+g^{\alpha \beta} \rho^{2} \partial_{\alpha} \psi \partial_{\beta} \psi+m^{2} \rho^{2}\right)^{2} \\
& -\frac{\Lambda^{4}}{8\left(\Lambda_{c}^{2}+\frac{1}{2} \rho^{2}\right)^{6}}\left[\left(\partial_{\eta} g^{\eta \nu}\right) \partial_{\nu} \rho+g^{\eta \nu} \partial_{\eta}\left(\partial_{\nu} \rho\right)\right] \\
& \times\left(g^{\alpha \beta} \partial_{\alpha} \rho \partial_{\beta} \rho+g^{\alpha \beta} \rho^{2} \partial_{\alpha} \psi \partial_{\beta} \psi+m^{2} \rho^{2}\right)^{2} \\
& -\frac{\Lambda^{4}}{4\left(\Lambda_{c}^{2}+\frac{1}{2} \rho^{2}\right)^{6}} g^{\eta \nu} \partial_{\nu} \rho\left[\left(\partial_{\eta} g^{\alpha \beta}\right) \partial_{\alpha} \rho \partial_{\beta} \rho+g^{\alpha \beta} \partial_{\eta}\left(\partial_{\alpha} \rho\right) \partial_{\beta} \rho+g^{\alpha \beta} \partial_{\alpha} \rho \partial_{\eta}\left(\partial_{\beta} \rho\right)\right. \\
& +\left(\partial_{\eta} g^{\alpha \beta}\right) \rho^{2} \partial_{\alpha} \psi \partial_{\beta} \psi+g^{\alpha \beta} \partial_{\eta}\left(\rho^{2}\right) \partial_{\alpha} \psi \partial_{\beta} \psi+g^{\alpha \beta} \rho^{2} \partial_{\eta}\left(\partial_{\alpha} \psi\right) \partial_{\beta} \psi \\
& \left.+g^{\alpha \beta} \rho^{2} \partial_{\alpha} \psi \partial_{\eta}\left(\partial_{\beta} \psi\right)+m^{2} \partial_{\eta}\left(\rho^{2}\right)\right] \\
& \times\left(g^{\omega \sigma} \partial_{\omega} \rho \partial_{\sigma} \rho+g^{\omega \sigma} \rho^{2} \partial_{\omega} \psi \partial_{\sigma} \psi+m^{2} \rho^{2}\right)
\end{aligned}
$$




$$
\begin{aligned}
= & -\frac{1}{2} \ddot{\rho}+\frac{3 \Lambda^{4} \rho \dot{\rho}^{2}}{4\left(\Lambda_{c}^{2}+\frac{1}{2} \rho^{2}\right)^{7}}\left(\dot{\rho}^{2}-\rho^{2} \mu^{2}+\rho^{2} \frac{n^{2}}{r^{2}}+m^{2} \rho^{2}\right)^{2} \\
& -\frac{\Lambda^{4}}{8\left(\Lambda_{c}^{2}+\frac{1}{2} \rho^{2}\right)^{6}} \ddot{\rho}\left(\dot{\rho}^{2}-\rho^{2} \mu^{2}+\rho^{2} \frac{n^{2}}{r^{2}}+m^{2} \rho^{2}\right)^{2} \\
& -\frac{\Lambda^{4} \dot{\rho}}{4\left(\Lambda_{c}^{2}+\frac{1}{2} \rho^{2}\right)^{6}}\left[\ddot{\rho} \dot{\rho}+\dot{\rho} \ddot{\rho}-2 \frac{n^{2}}{r^{3}} \rho^{2}-2 \rho \dot{\rho} \mu^{2}+2 \rho \dot{\rho} \frac{n^{2}}{r^{2}}+2 m^{2} \rho \dot{\rho}\right] \\
\quad \times\left(\dot{\rho}^{2}-\rho^{2} \mu^{2}+\rho^{2} \frac{n^{2}}{r^{2}}+m^{2} \rho^{2}\right) & -\frac{1}{2} \ddot{\rho}+\frac{3 \Lambda^{4} \rho \dot{\rho}^{2}}{4\left(\Lambda_{c}^{2}+\frac{1}{2} \rho^{2}\right)^{7}}\left(\dot{\rho}^{2}+q \rho^{2}\right)^{2}-\frac{\Lambda^{4}}{8\left(\Lambda_{c}^{2}+\frac{1}{2} \rho^{2}\right)^{6}} \ddot{\rho}\left(\dot{\rho}^{2}+q \rho^{2}\right)^{2} \\
& -\frac{\Lambda^{4} \dot{\rho}}{4\left(\Lambda_{c}^{2}+\frac{1}{2} \rho^{2}\right)^{6}}\left[2 \dot{\rho} \ddot{\rho}-2 \frac{n^{2}}{r^{3}} \rho^{2}+2 q \rho \dot{\rho}\right]\left(\dot{\rho}^{2}+q \rho^{2}\right) .
\end{aligned}
$$

Inserting equation (A.27) and (A.31) into equation (A.16) gives

$$
\begin{aligned}
0= & -\frac{1}{2} q \rho+\frac{\Lambda^{4} \rho}{8\left(\Lambda_{c}^{2}+\frac{1}{2} \rho^{2}\right)^{7}}\left(\dot{\rho}^{2}+q \rho^{2}\right)^{3}-\frac{\Lambda^{4}}{8\left(\Lambda_{c}^{2}+\frac{1}{2} \rho^{2}\right)^{6}} q \rho\left(\dot{\rho}^{2}+q \rho^{2}\right)^{2} \\
& +\frac{1}{2} \ddot{\rho}-\frac{3 \Lambda^{4} \rho \dot{\rho}^{2}}{4\left(\Lambda_{c}^{2}+\frac{1}{2} \rho^{2}\right)^{7}}\left(\dot{\rho}^{2}+q \rho^{2}\right)^{2}+\frac{\Lambda^{4}}{8\left(\Lambda_{c}^{2}+\frac{1}{2} \rho^{2}\right)^{6}} \ddot{\rho}\left(\dot{\rho}^{2}+q \rho^{2}\right)^{2} \\
& +\frac{\Lambda^{4} \dot{\rho}}{4\left(\Lambda_{c}^{2}+\frac{1}{2} \rho^{2}\right)^{6}}\left[2 \dot{\rho} \ddot{\rho}-2 \frac{n^{2}}{r^{3}} \rho^{2}+2 q \rho \dot{\rho}\right]\left(\dot{\rho}^{2}+q \rho^{2}\right) .
\end{aligned}
$$

We now want to find an expression for $\ddot{\rho}$, so that equation (A.32) may be solved numerically (hopefully). Let us dive right in ${ }^{1}$ :

$$
\begin{aligned}
0= & -\frac{1}{2} q \rho+\frac{\Lambda^{4} \rho}{8\left(\Lambda_{c}^{2}+\frac{1}{2} \rho^{2}\right)^{7}}\left(\dot{\rho}^{2}+q \rho^{2}\right)^{3}-\frac{\Lambda^{4}}{8\left(\Lambda_{c}^{2}+\frac{1}{2} \rho^{2}\right)^{6}} q \rho\left(\dot{\rho}^{2}+q \rho^{2}\right)^{2} \\
& +\frac{1}{2} \ddot{\rho}-\frac{3 \Lambda^{4} \rho \dot{\rho}^{2}}{4\left(\Lambda_{c}^{2}+\frac{1}{2} \rho^{2}\right)^{7}}\left(\dot{\rho}^{2}+q \rho^{2}\right)^{2}+\frac{\Lambda^{4}}{8\left(\Lambda_{c}^{2}+\frac{1}{2} \rho^{2}\right)^{6}} \ddot{\rho}\left(\dot{\rho}^{2}+q \rho^{2}\right)^{2} \\
& +\frac{\Lambda^{4} \dot{\rho}}{4\left(\Lambda_{c}^{2}+\frac{1}{2} \rho^{2}\right)^{6}}\left[2 \dot{\rho} \ddot{\rho}-2 \frac{n^{2}}{r^{3}} \rho^{2}+2 q \rho \dot{\rho}\right]\left(\dot{\rho}^{2}+q \rho^{2}\right)
\end{aligned}
$$

\footnotetext{
${ }^{1}$ This will all be over very soon.
} 


$$
\begin{aligned}
& 0=-4 q \rho\left(\Lambda_{c}^{2}+\frac{1}{2} \rho^{2}\right)^{7}+\Lambda^{4} \rho\left(\dot{\rho}^{2}+q \rho^{2}\right)^{3}-\Lambda^{4}\left(\Lambda_{c}^{2}+\frac{1}{2} \rho^{2}\right) q \rho\left(\dot{\rho}^{2}+q \rho^{2}\right)^{2} \\
& +4 \ddot{\rho}\left(\Lambda_{c}^{2}+\frac{1}{2} \rho^{2}\right)^{7}-6 \Lambda^{4} \rho \dot{\rho}^{2}\left(\dot{\rho}^{2}+q \rho^{2}\right)^{2}+\Lambda^{4}\left(\Lambda_{c}^{2}+\frac{1}{2} \rho^{2}\right) \ddot{\rho}\left(\dot{\rho}^{2}+q \rho^{2}\right)^{2} \\
& +2 \Lambda^{4}\left(\Lambda_{c}^{2}+\frac{1}{2} \rho^{2}\right) \dot{\rho}\left[2 \dot{\rho} \ddot{\rho}-2 \frac{n^{2}}{r^{3}} \rho^{2}+2 q \rho \dot{\rho}\right]\left(\dot{\rho}^{2}+q \rho^{2}\right) \\
& 0=-4 q \rho\left(\Lambda_{c}^{2}+\frac{1}{2} \rho^{2}\right)^{7}+\Lambda^{4} \rho\left(\dot{\rho}^{2}+q \rho^{2}\right)\left(\dot{\rho}^{4}+2 q \rho^{2} \dot{\rho}^{2}+q^{2} \rho^{4}\right) \\
& -\Lambda^{4}\left(\Lambda_{c}^{2}+\frac{1}{2} \rho^{2}\right) q \rho\left(\dot{\rho}^{4}+2 q \dot{\rho}^{2} \rho^{2}+q^{2} \rho^{4}\right)+4 \ddot{\rho}\left(\Lambda_{c}^{2}+\frac{1}{2} \rho^{2}\right)^{7} \\
& -6 \Lambda^{4} \rho \dot{\rho}^{2}\left(\dot{\rho}^{4}+2 q \rho^{2} \dot{\rho}^{2}+q^{2} \rho^{4}\right)+\Lambda^{4}\left(\Lambda_{c}^{2}+\frac{1}{2} \rho^{2}\right) \ddot{\rho}\left(\dot{\rho}^{4}+2 q \rho^{2} \dot{\rho}^{2}+q^{2} \rho^{4}\right) \\
& +2 \Lambda^{4}\left(\Lambda_{c}^{2}+\frac{1}{2} \rho^{2}\right) \dot{\rho}\left[2 \dot{\rho}^{3} \ddot{\rho}+2 \dot{\rho} \ddot{\rho} \rho^{2} q-2 \frac{n^{2}}{r^{3}} \rho^{2} \dot{\rho}^{2}-2 \rho^{4} q \frac{n^{2}}{r^{3}}+2 q \rho \dot{\rho}^{3}+2 \rho^{3} \dot{\rho} q^{2}\right] \\
& 0=-4 q \rho\left(\Lambda_{c}^{2}+\frac{1}{2} \rho^{2}\right)^{7}+\Lambda^{4} \rho\left(\dot{\rho}^{6}+2 q \rho^{2} \dot{\rho}^{4}+q^{2} \rho^{4} \dot{\rho}^{2}+q \rho^{2} \dot{\rho}^{4}+2 q^{2} \rho^{4} \dot{\rho}^{2}+q^{3} \rho^{6}\right) \\
& -\Lambda^{4}\left(\Lambda_{c}^{2}+\frac{1}{2} \rho^{2}\right)\left(q \rho \dot{\rho}^{4}+2 q^{2} \dot{\rho}^{2} \rho^{3}+q^{3} \rho^{5}\right)+4 \ddot{\rho}\left(\Lambda_{c}^{2}+\frac{1}{2} \rho^{2}\right)^{7}-6 \Lambda^{4} \rho \dot{\rho}^{6} \\
& -12 \Lambda^{4} q \rho^{3} \dot{\rho}^{4}-6 \Lambda^{4} q^{2} \rho^{5} \dot{\rho}^{2}+\left(\Lambda_{c}^{2}+\frac{1}{2} \rho^{2}\right) \ddot{\rho}\left(\Lambda^{4} \dot{\rho}^{4}+2 \Lambda^{4} q \rho^{2} \dot{\rho}^{2}+\Lambda^{4} q^{2} \rho^{4}\right) \\
& +\left(\Lambda_{c}^{2}+\frac{1}{2} \rho^{2}\right)\left[4 \Lambda^{4} \dot{\rho}^{4} \ddot{\rho}+4 \Lambda^{4} q \rho^{2} \dot{\rho}^{2} \ddot{\rho}-4 \Lambda^{4} \rho^{2} \dot{\rho}^{3} \frac{n^{2}}{r^{3}}-4 \Lambda^{4} q \rho^{4} \dot{\rho} \frac{n^{2}}{r^{3}}+4 \Lambda^{4} q \rho \dot{\rho}^{4}+4 \Lambda^{4} q^{2} \rho^{3} \dot{\rho}^{2}\right] \\
& 0=-4 q \rho\left(\Lambda_{c}^{2}+\frac{1}{2} \rho^{2}\right)^{7}+\Lambda^{4} \rho \dot{\rho}^{6}+2 \Lambda^{4} q \rho^{3} \dot{\rho}^{4}+\Lambda^{4} q^{2} \rho^{5} \dot{\rho}^{2}+\Lambda^{4} q \rho^{3} \dot{\rho}^{4}+2 \Lambda^{4} q^{2} \rho^{5} \dot{\rho}^{2}+\Lambda^{4} q^{3} \rho^{7} \\
& -\left(\Lambda_{c}^{2}+\frac{1}{2} \rho^{2}\right)\left(\Lambda^{4} q \rho \dot{\rho}^{4}+2 \Lambda^{4} q^{2} \rho^{3} \dot{\rho}^{2}+\Lambda^{4} q^{3} \rho^{5}\right)+4 \ddot{\rho}\left(\Lambda_{c}^{2}+\frac{1}{2} \rho^{2}\right)^{7}-6 \Lambda^{4} \rho \dot{\rho}^{6} \\
& -12 \Lambda^{4} q \rho^{3} \dot{\rho}^{4}-6 \Lambda^{4} q^{2} \rho^{5} \dot{\rho}^{2}+\left(\Lambda_{c}^{2}+\frac{1}{2} \rho^{2}\right) \ddot{\rho}\left(\Lambda^{4} \dot{\rho}^{4}+2 \Lambda^{4} q \rho^{2} \dot{\rho}^{2}+\Lambda^{4} q^{2} \rho^{4}\right) \\
& +\left(\Lambda_{c}^{2}+\frac{1}{2} \rho^{2}\right)\left[4 \Lambda^{4} \dot{\rho}^{4} \ddot{\rho}+4 \Lambda^{4} q \rho^{2} \dot{\rho}^{2} \ddot{\rho}-4 \Lambda^{4} \rho^{2} \dot{\rho} \frac{n^{2}}{r^{3}}\left(\dot{\rho}^{2}+q \rho^{2}\right)+4 \Lambda^{4} q \rho \dot{\rho}^{4}+4 \Lambda^{4} q^{2} \rho^{3} \dot{\rho}^{2}\right] \\
& 0=-4 q \rho\left(\Lambda_{c}^{2}+\frac{1}{2} \rho^{2}\right)^{7}-5 \Lambda^{4} \rho \dot{\rho}^{6}-9 \Lambda^{4} q \rho^{3} \dot{\rho}^{4}-3 \Lambda^{4} q^{2} \rho^{5} \dot{\rho}^{2}+\Lambda^{4} q^{3} \rho^{7} \\
& +\left(\Lambda_{c}^{2}+\frac{1}{2} \rho^{2}\right)\left(-\Lambda^{4} q \rho \dot{\rho}^{4}-2 \Lambda^{4} q^{2} \rho^{3} \dot{\rho}^{2}-\Lambda^{4} q^{3} \rho^{5}-4 \Lambda^{4} \rho^{2} \dot{\rho} \frac{n^{2}}{r^{3}}\left(\dot{\rho}^{2}+q \rho^{2}\right)+4 \Lambda^{4} q \rho \dot{\rho}^{4}+4 \Lambda^{4} q^{2} \rho^{3} \dot{\rho}^{2}\right) \\
& +\ddot{\rho}\left(\Lambda_{c}^{2}+\frac{1}{2} \rho^{2}\right)\left(4\left(\Lambda_{c}^{2}+\frac{1}{2} \rho^{2}\right)^{6}+\Lambda^{4} \dot{\rho}^{4}+2 \Lambda^{4} q \rho^{2} \dot{\rho}^{2}+\Lambda^{4} q^{2} \rho^{4}+4 \Lambda^{4} \dot{\rho}^{4}+4 \Lambda^{4} q \rho^{2} \dot{\rho}^{2}\right)
\end{aligned}
$$




$$
\begin{aligned}
0= & -4 q \rho\left(\Lambda_{c}^{2}+\frac{1}{2} \rho^{2}\right)^{7}-5 \Lambda^{4} \rho \dot{\rho}^{6}-9 \Lambda^{4} q \rho^{3} \dot{\rho}^{4}-3 \Lambda^{4} q^{2} \rho^{5} \dot{\rho}^{2}+\Lambda^{4} q^{3} \rho^{7} \\
& +\left(\Lambda_{c}^{2}+\frac{1}{2} \rho^{2}\right)\left(3 \Lambda^{4} q \rho \dot{\rho}^{4}+2 \Lambda^{4} q^{2} \rho^{3} \dot{\rho}^{2}-\Lambda^{4} q^{3} \rho^{5}-4 \Lambda^{4} \rho^{2} \dot{\rho} \frac{n^{2}}{r^{3}}\left(\dot{\rho}^{2}+q \rho^{2}\right)\right) \\
& +\ddot{\rho}\left(\Lambda_{c}^{2}+\frac{1}{2} \rho^{2}\right)\left(4\left(\Lambda_{c}^{2}+\frac{1}{2} \rho^{2}\right)^{6}+5 \Lambda^{4} \dot{\rho}^{4}+6 \Lambda^{4} q \rho^{2} \dot{\rho}^{2}+\Lambda^{4} q^{2} \rho^{4}\right) .
\end{aligned}
$$

This finally leads to an expression for $\ddot{\rho}$, given as

$$
\ddot{\rho}=\frac{f(r, \rho, \dot{\rho})}{g(r, \rho, \dot{\rho})},
$$

where

$$
\begin{aligned}
f(r, \rho, \dot{\rho})= & 5 \rho \dot{\rho}^{6}+9 q \rho^{3} \dot{\rho}^{4}+3 q^{2} \rho^{5} \dot{\rho}^{2}-q^{3} \rho^{7}-\left(\Lambda_{c}+\frac{1}{2} \rho^{2}\right)\left[-\frac{4 q}{\Lambda^{4}} \rho\left(\Lambda_{c}+\frac{1}{2} \rho^{2}\right)^{6}\right. \\
& \left.+3 q \rho \dot{\rho}^{4}+2 q^{2} \rho^{3} \dot{\rho}^{2}-q^{3} \rho^{5}-4 \rho^{2} \dot{\rho} \frac{n^{2}}{r^{3}}\left(\dot{\rho}^{2}+q \rho^{2}\right)\right] \\
g(r, \rho, \dot{\rho})= & \left(\Lambda_{c}+\frac{1}{2} \rho^{2}\right)\left[\frac{4}{\Lambda^{4}}\left(\Lambda_{c}+\frac{1}{2} \rho^{2}\right)^{6}+5 \dot{\rho}^{4}+6 q \rho^{2} \dot{\rho}^{2}+q^{2} \rho^{4}\right] .
\end{aligned}
$$

To make the expression easier to work with, we will write it on a unitless form. To do so, we introduce the unitless variables

$$
R=\rho / \rho_{0} \quad \text { and } \quad \eta=\rho_{0} \mathrm{r},
$$

similar to those presented in equation (4.29). In this case we also want $\rho_{0}$ to be the solution to the Euler-Lagrange equations with respect to $\rho$ when $\nabla \rho=\nabla \psi=0$. If we consider the full Lagrangian, it is quite difficult to reach a simple expression for $\rho_{0}$. Instead, we will therefore consider the case $\Lambda_{c}^{2} \ll|\phi|^{2}$, defined as the MOND regime in [1]. This is equivalent to putting $\Lambda_{c}=0$ in our equations, and we get

$$
\begin{aligned}
\mathscr{L}= & -\frac{1}{4}\left(g^{\mu \nu} \partial_{\mu} \rho \partial_{\nu} \rho+g^{\mu \nu} \rho^{2} \partial_{\mu} \psi \partial_{\nu} \psi+m^{2} \rho^{2}\right) \\
& -\frac{\Lambda^{4}}{48\left(\Lambda_{c}^{2}+\frac{1}{2} \rho^{2}\right)^{6}}\left(g^{\mu \nu} \partial_{\mu} \rho \partial_{\nu} \rho+g^{\mu \nu} \rho^{2} \partial_{\mu} \psi \partial_{\nu} \psi+m^{2} \rho^{2}\right)^{3} \\
= & -\frac{1}{4}\left(-\rho^{2} \mu^{2}+m^{2} \rho^{2}\right)-\frac{4 \Lambda^{4}}{3 \rho^{12}}\left(-\rho^{2} \mu^{2}+m^{2} \rho^{2}\right)^{3} \\
= & -\frac{1}{4}\left(m^{2}-\mu^{2}\right) \rho^{2}-\frac{4 \Lambda^{4}}{3}\left(m^{2}-\mu^{2}\right)^{3} \frac{1}{\rho^{6}}
\end{aligned}
$$


which results in the Euler-Lagrange equation

$$
\begin{aligned}
0 & =\frac{\partial \mathscr{L}}{\partial \rho} \\
0 & =-\frac{1}{2}\left(m^{2}-\mu^{2}\right) \rho+8 \Lambda^{4}\left(m^{2}-\mu^{2}\right)^{3} \frac{1}{\rho^{7}} \\
\rho^{8} & =16 \Lambda^{4}\left(m^{2}-\mu^{2}\right)^{2} \\
\rho & =\sqrt{2 \Lambda}\left(m^{2}-\mu^{2}\right)^{1 / 4},
\end{aligned}
$$

meaning that

$$
\rho_{0}=\sqrt{2 \Lambda}\left(m^{2}-\mu^{2}\right)^{1 / 4} .
$$

From the new variables, we need to change the following:

$$
\rho \rightarrow \rho_{0} R, \quad \dot{\rho} \rightarrow \rho_{0}^{2} \dot{R} \quad \text { and } \quad \ddot{\rho} \rightarrow \rho_{0}^{3} \ddot{\mathrm{R}}
$$

where a dot now symbolizes the derivative with respect to $\eta$. In addition, we have

$$
\begin{aligned}
q & =m^{2}-\mu^{2}+\frac{n^{2}}{r^{2}} \\
& \rightarrow \frac{\rho_{0}^{4}}{4 \Lambda^{2}}+\frac{\rho_{0}^{2} n^{2}}{\eta^{2}} \\
& =\rho_{0}^{2}\left(\frac{\rho_{0}^{2}}{4 \Lambda^{2}}+\frac{n^{2}}{\eta^{2}}\right) \\
& =\rho_{0}^{2} p .
\end{aligned}
$$

Let us now insert the new variables into equation (A.41), term by term:
(1) $5 \rho \dot{\rho}^{6}$
$=5 \rho_{0}^{13} R \dot{R}^{6}$,
(2) $9 q \rho^{3} \dot{\rho}^{4}$
$=9 p \rho_{0}^{13} R^{3} \dot{R}^{4}$
(3) $3 q^{2} \rho^{5} \dot{\rho}^{2}$
$=3 p^{2} \rho_{0}^{13} R^{5} \dot{R}^{2}$,
(4) $-q^{3} \rho^{7}$
$=-p^{3} \rho_{0}^{13} R^{7}$,

and then there is the factor

$$
-\left(\Lambda_{c}^{2}+\frac{1}{2} \rho^{2}\right)=-\left(\Lambda_{c}^{2}+\frac{1}{2} \rho_{0}^{2} R^{2}\right)
$$

multiplied by the terms
(5) $-4 q \rho\left(\Lambda_{c}^{2}+\frac{1}{2} \rho^{2}\right)^{6}$
$=-4 p \rho_{0}^{3} R\left(\Lambda_{c}^{2}+\frac{1}{2} \rho_{0}^{2} R^{2}\right)$,
(6) $3 q \rho \dot{\rho}^{4}$
$=3 p \rho_{0}^{11} R \dot{R}^{4}$, 

(7) $2 q^{2} \rho^{3} \dot{\rho}^{2}$
$=2 p^{2} \rho_{0}^{11} R^{3} \dot{R}^{2}$,
(8) $-q^{3} \rho^{5}$
$=-p^{3} \rho_{0}^{11} R^{5}$,
(9) $-4 \rho^{2} \dot{\rho} \frac{n^{2}}{r^{3}}\left(\dot{\rho}^{2}+q \rho^{2}\right)$
$=-4 \rho_{0}^{11} R^{2} \dot{R} \frac{n^{2}}{\eta^{3}}\left(\dot{R}^{2}+p R^{2}\right)$.

For equation (A.42), we have the factor in equation (A.61) (without the negative sign) multiplying the terms
(10) $4\left(\Lambda_{c}^{2}+\frac{1}{2} \rho^{2}\right)^{6}$
$=4\left(\Lambda_{c}^{2}+\frac{1}{2} \rho_{0}^{2} R^{2}\right)^{6}$,
(11) $5 \dot{\rho}^{4}$
$=5 \rho_{0}^{8} \dot{R}^{4}$,
(12) $6 q \rho^{2} \dot{\rho}^{2}$
$=6 p \rho_{0}^{8} R^{2} \dot{R}^{2}$,
(13) $q^{2} \rho^{4}$
$=p^{2} \rho_{0}^{8} R^{4}$.

All in all, factoring out a few $\rho_{0}$ 's, we get

$$
\ddot{R}=\frac{\bar{f}(\eta, R, \dot{R})}{\bar{g}(\eta, R, \dot{R})},
$$

where

$$
\begin{aligned}
\bar{f}(\eta, R, \dot{R})= & \rho_{0}^{2}\left(5 R \dot{R}^{6}+9 p R^{3} \dot{R}^{4}+3 p^{2} R^{5} \dot{R}^{2}-p^{3} R^{7}\right) \\
& -\left(\Lambda_{c}^{2}+\frac{1}{2} \rho_{0}^{2} R^{2}\right)\left[-\frac{4 p}{\Lambda^{4} \rho_{0}^{8}} R\left(\Lambda_{c}^{2}+\frac{1}{2} \rho_{0}^{2} R^{2}\right)^{6}\right. \\
& \left.+3 p R \dot{R}^{4}+2 p^{2} R^{3} \dot{R}^{2}-p^{3} R^{5}-4 R^{2} \dot{R} \frac{n^{2}}{\eta^{3}}\left(\dot{R}^{2}+p R^{2}\right)\right] \\
\bar{g}(\eta, R, \dot{R})= & \left(\Lambda_{c}^{2}+\frac{1}{2} \rho_{0}^{2} R^{2}\right)\left[\frac{4}{\Lambda^{4} \rho_{0}^{8}}\left(\Lambda_{c}^{2}+\frac{1}{2} \rho_{0}^{2} R^{2}\right)^{6}+5 \dot{R}^{4}+6 p R^{2} \dot{R}^{2}+p^{2} R^{4}\right] .
\end{aligned}
$$




\section{Appendix B}

\section{Numerical Methods}

In this appendix we explain some of the numerical methods used when trying to solve the vortex equation of chapter 8.

\section{B.1 The Shooting Method}

If you ask the Internet for advice when it comes to solving a boundary value problem, the most favored answer seems to be the shooting method. Based on this, and also advice from more experienced researchers $[76,88]$, this method quickly became the main focus when trying to solve equation (8.37). In this section, we will therefore give a quick and simple introduction to the motivation behind the given method and how it works.

The idea behind the shooting method is to convert a boundary value problem (BVP) to a system of initial value problems (IVPs). The difference between the two types of problems is what we know about the properties of the solution before actually solving the given differential equation. For a BVP, we know something about the behavior of the solution at the boundaries. In our case, this is given as $\rho(r=0)=0$ and $\rho(r \rightarrow \infty) \rightarrow \mathrm{K}$, where $K$ is a constant. For an IVP, we know the value of the solution and its derivative at some point. In our case, that would mean $\rho(r=0)=0$ and $\frac{d \rho}{d r}(r=0)=\omega$, where $\omega$ is known. Having the value of the solution at the center, along with the derivative at the center, enables us to use all the well-known methods for solving IVPs, such as different Euler schemes or Runge-Kutta methods. Sadly, $\omega$ is unknown to us, and this is where the shooting method makes its grand entrance.

Simply put, the shooting method lets us guess at the missing initial value, solve the given differential equation using one of the well-known IVP-solvers, and then check how well our solution matches the given boundary conditions. If the solution matches to within a given tolerance, the shooting is successful, and we have our solution. If not, we make a new guess and try again. Different algorithms may be used to make new guesses for the initial value, so that the solution should converge sooner or later.

Let us now outline the procedure, closely following the explanation given in [89]. 
Imagine having a second order ODE on the form

$$
y^{\prime \prime}=f\left(x, y, y^{\prime}\right),
$$

defined on the interval $a \leq x \leq b$, with the boundary values

$$
y(a)=\alpha, \quad y(b)=\beta .
$$

First of all, this must be reduced to a system of first-order equations. This can be done by defining the following equations, with corresponding initial values:

$$
\begin{aligned}
y^{\prime} & =\frac{d y}{d x}=z, & y(a) & =\alpha \\
y^{\prime \prime} & =\frac{d z}{d x}=f(x, y, z), & z(a) & =y^{\prime}(a)=s_{i} .
\end{aligned}
$$

Here $s_{i}$ symbolizes several real numbers, meaning that for each $i$ we will make a guess at $y^{\prime}(a)$. The solution we obtain, $y$, will be dependent upon $s_{i}$, so we may write $y=y\left(x, s_{i}\right)$. The point of the shooting method is to, for each $i$, make a guess at $y^{\prime}(a)$ so that

$$
\lim _{i \rightarrow \infty} y\left(x, s_{i}\right)=y(x),
$$

where $y(x)$ is the solution fulfilling the original boundary conditions. To check how well our guess at the initial value is (that is $s_{i}$ ), we compare to the given boundary value, $\beta$, and the point $x=b$. In this way, $y\left(b, s_{i}\right)-\beta$ is the resulting error of the given $s_{i}$ guess. There should exist some $s_{i}$ value, let's call it $s$, that makes the error zero:

$$
y(s, b)-\beta=0 .
$$

Finding the $s$-value of equation (B.6) is now a root finding problem, to which there are many known numerical methods, such as the secant method or Newton's method. We will take a closer look at the latter in section B.3. Anyway, the point is that we may now make a mathematical guess at the initial slope of our solution, ensuring convergence in most cases. All in all, this means that we will make some initial guess of $y^{\prime}(a)$, solve the IVP, check the error, and if not within a given tolerance, guess the value $y^{\prime}(a)$ again, based on some numerical root finding method. The shooting method works for both linear and non-linear ordinary differential equations, although more iterations are usually required for the non-linear case.

Let us finish off this section with a few comments regarding the use of the shooting method on our specific equation. When implementing the method, we followed the procedure outlined in section 11.2 of [90]. Here, a 4th order Runge-Kutta method is used to solve the IVP, while Newton's method is used to make a guess at new values of 
$y^{\prime}(a)=s_{i}$. The problem we face is that, for our very first guess at the missing initial condition, the solution quickly diverges and gives a numerical overflow (in the same way as illustrated in figure 8.2a). This prevents us from ever using Newton's method to make a better guess at the needed initial condition. It seems like our very first guess must be incredibly accurate to even allow for the algorithm to run. This is very inconvenient and again points towards our equation being quite unstable. As mentioned in [91], some initial value problems will be unstable when we use the shooting method, even though the BVP is well defined. One possibility for the future could be to try out some finite difference or collocation methods, which according to [91] might be more robust.

\section{B.2 The solve_bvp Module}

To solve our original vortex equation, namely equation (4.36) from section 4.3.1, we used an inbuilt SciPy module, solve_bvp [58]. For the given equation, the inbuilt module worked well and nicely reproduced already known results. It was therefore natural to check if the module would work for us yet again. For the module to work, an initial guess of the solution is required, and as we want a vortex solution, the guess $R(\eta)=\eta / \sqrt{2+\eta^{2}}$ was used. As you might suspect, the solve_bvp module is not able to solve our slightly more advanced vortex equation (8.37). The algorithm finishes with an error message, stating that there does not exist any converging solution for the given initial guess. Now, this indicates that there might exist some solution, with a different behavior, if we only make a better initial guess. However, the possible guesses are endless, and there are no clear indications of where to start. It is therefore more likely that a self-written code will give us what we need.

An inbuilt module could be very thoroughly made and account for many different scenarios, which would take you a long time to incorporate in your own code. However, there is an extra motivation behind writing the algorithm to solve the equation yourself. When using a built-in module, obtaining an understanding of what actually goes on can be quite difficult. This makes it hard to know if the results are good, unless you already have something you can compare them too, which was the case for us regarding equation (4.36).

For the sake of order, we will try to briefly explain how the solve_bvp module works. First of all, the algorithm requires that we rewrite our original equation into a system of first-order differential equations and present the resulting equations in a function. Then we need a function to handle the boundary conditions and an initial guess. For specific examples on how to use the module, see [58]. As also stated in the aforementioned source, the solve_bvp module implements a 4th order collocation algorithm and uses a damped Newton method to solve the resulting system. Let us put into words what this means.

Collocation is a method where one chooses a function from some simple, finitedimensional space (usually a polynomial), along with a set of points, known as collocation points. One then requires that the chosen function satisfies the given ordinary 
differential equation (ODE) at the collocation points [92]. A collocation method is in fact an implicit Runge-Kutta method, but not all implicit Runge-Kutta methods are collocation methods. Newton's method, which we will look at in more detail in section B.3, is a root finding method. This means that it tries to find the zero of some given function. As with all numerical methods, one has to start at an initial point or value. In some cases, where you choose to start from will determine if Newton's method converges towards a solution or not. For the damped Newton method, the algorithm should converge no matter the starting point [93].

The full solve_bvp code is available at [94].

\section{B.3 Newton's Method}

This section is based on a code and method (Newton's method with finite difference) written by John Skorgan [95], a fellow master student at the Institute of Theoretical Astrophysics (UiO). The code was developed for his own project, but he was kind enough to test it for our specific case as well (equation 8.37).

Let us briefly explain how the method works. As stated before, Newton's method is a root finding method. For a function $f$, one makes an initial guess at the root, say $x_{0}$. If this is not the value of the root, Newton's method will choose

$$
x_{1}=x_{0}-\frac{f\left(x_{0}\right)}{f^{\prime}\left(x_{0}\right)}
$$

as a better approximation. What essentially happens is that the new value of the root is chosen as the point where the tangent of the function in the previous point hits the $x$-axis. This process is repeated until a sufficient value, within some tolerance, is found:

$$
x_{i+1}=x_{i}-\frac{f\left(x_{i}\right)}{f^{\prime}\left(x_{i}\right)}
$$

This expression originates from the Taylor expansion (to first order) of a function around $x_{i}$, where $x_{i+1}=x_{i}+h$ and $h$ is some small step:

$$
f\left(x_{i+1}\right)=f\left(x_{i}\right)+\left(x_{i+1}-x_{i}\right) f^{\prime}\left(x_{i}\right)
$$

By putting $f\left(x_{i+1}\right)=0$ we get equation (B.8), showing that this expression will indeed find the zero of the function.

The method may be written on matrix form. First, let us introduce $\boldsymbol{y}^{(i)}$, an $N \times 1$ vector, and $\boldsymbol{f}\left(\boldsymbol{y}^{(i)}\right)$ as another vector of the same dimensions. Let us then denote the derivative of $\boldsymbol{f}\left(\boldsymbol{y}^{(i)}\right)$ by

$$
\boldsymbol{f}^{\prime}\left(\boldsymbol{y}^{(i)}\right)=\boldsymbol{J}\left(\boldsymbol{y}^{(i)}\right),
$$


where $\boldsymbol{J}\left(\boldsymbol{y}^{(i)}\right)$ now is an $N \times N$ Jacobian matrix with elements

$$
\boldsymbol{J}\left(\boldsymbol{x}^{(i)}\right)=\left[\begin{array}{cccc}
\frac{\partial f_{1}}{\partial y_{1}} & \frac{\partial f_{1}}{\partial y_{2}} & \ldots & \frac{\partial f_{1}}{\partial y_{N}} \\
\frac{\partial f_{2}}{\partial y_{1}} & \frac{\partial f_{2}}{\partial y_{2}} & \ldots & \frac{\partial f_{2}}{\partial y_{N}} \\
\vdots & \ddots & \ddots & \vdots \\
\frac{\partial f_{N}}{\partial y_{1}} & \frac{\partial f_{N}}{\partial y_{2}} & \ldots & \frac{\partial f_{N}}{\partial y_{N}}
\end{array}\right]
$$

With these new variables, we may rewrite equation (B.8) as

$$
\boldsymbol{y}^{(i+1)}=\boldsymbol{y}^{(i)}-\left[\boldsymbol{J}\left(\boldsymbol{y}^{(i)}\right)\right]^{-1} \boldsymbol{f}\left(\boldsymbol{y}^{(i)}\right) .
$$

From equation (B.8) we can see that, when the method has converged, we should get $x_{i+1}=x_{i}$, meaning that $f\left(x_{i}\right)=0$. In the case of equation (B.12), when performing this numerically, we want $\left|\boldsymbol{y}^{(i-1)}-\boldsymbol{y}^{(i)}\right|<\epsilon$, where $\epsilon$ is some given tolerance. This should be the same as $\boldsymbol{f}\left(\boldsymbol{y}^{(i)}\right)=\mathbf{0}$.

In general, we will have some ordinary differential equation that needs solving. This is an equation that usually involves the first and/or second derivative of the function, $f$, we want to find. One way to incorporate that into Newton's method is by finite difference, which is an expression on the form $f(x+b)-f(x+a)$. For example, we may Taylor expand $f(x+h)$ and $f(x-h)$ up to second order,

$$
\begin{aligned}
& f(x+h) \simeq f(x)+h f^{\prime}(x)+\frac{h^{2}}{2} f^{\prime \prime}(x)(x) \\
& f(x-h) \simeq f(x)-h f^{\prime}(x)+\frac{h^{2}}{2} f^{\prime \prime}(x)(x),
\end{aligned}
$$

and by adding/subtracting these expressions, gain discretized equations useful for the implementation of Newton's method. Subtracting equation (B.14) from equation (B.13), and defining $x+h=x_{i+1}$ and $x-h=x_{i-1}$, we obtain an expression for the derivative

$$
f^{\prime}(x)=\frac{f\left(x_{i+1}\right)-f\left(x_{i-1}\right)}{2 h} .
$$

Adding equation (B.14) and (B.13) we get

$$
-f(x+h)+2 f(x)-f(x-h)+h^{2} f^{\prime \prime}(x)=0,
$$


where we may assume $f^{\prime \prime}$ to be on the form

$$
f^{\prime \prime}\left(x_{i}\right)=Z\left(x_{i}, f\left(x_{i}\right), f^{\prime}\left(x_{i}\right)\right)=Z\left(x_{i}, f\left(x_{i}\right), \frac{f\left(x_{i+1}\right)-f\left(x_{i-1}\right)}{2 h}\right) .
$$

This results in the equation

$$
-f\left(x_{i+1}\right)+2 f\left(x_{i}\right)-f\left(x_{i-1}\right)+h^{2} Z\left(x_{i}, f\left(x_{i}\right), \frac{f\left(x_{i+1}\right)-f\left(x_{i-1}\right)}{2 h}\right)=0,
$$

which we can rewrite to fit Newton's method. If we redefine $f\left(x_{i}\right)=y_{i}$, we may treat equation (B.18) as our $\boldsymbol{f}\left(\boldsymbol{y}^{i}\right)$ function, meaning that we are looking for values of $\boldsymbol{y}$ so that $\boldsymbol{f}(\boldsymbol{y})=\mathbf{0}$. This can be solved by Newton's method. This does, however, require an initial guess, in the same way that you have to guess at a position of the root, $x_{0}$, in equation (B.7). In our case, this guess should include the boundary conditions of the function we are looking for. However, we do not know, in general, how the function behaves otherwise, and the simplest initial guess is therefore a linear function

$$
y_{i}=y_{0}+\left(\frac{y_{N+1}-y_{0}}{N+1}\right) i
$$

Here $i=0,1, \ldots, N+1$, and the boundary conditions are included as $f\left(x_{0}\right)=y_{0}$ and $f\left(y_{N+1}\right)=y_{N+1}$.

All in all, we now have a function

$$
f_{i}=-y_{i+1}+2 y_{i}-y_{i-1}+h^{2} Z\left(x_{i}, y_{i}, \frac{y_{i+1}-y_{i-1}}{2 h}\right),
$$

which is our $\boldsymbol{f}(\boldsymbol{y})$ equation. From this, we may calculate the Jacobian matrix of the system, which will depend on the ordinary differential equation we want to solve through the double derivative, $Z$.

Altogether, one would solve equation (B.12) with equation (B.20) as $\boldsymbol{f}\left(\boldsymbol{y}^{i}\right)$, checking, for each iteration, if $\left|\boldsymbol{y}^{i+1}-\boldsymbol{y}^{i}\right|<\epsilon$. If this is the case, we have our solution; if not, we continue until the solution converges.

For our vortex equation, calculating the Jacobian matrix is quite a mess, and the calculation will therefore not be included here. It suffices to say that this method did not solve our equation, as the output quickly overflowed and caused numerical problems. A possible next step, when time allows, could be to make a better initial guess. 


\section{Bibliography}

[1] L. Berezhiani and J. Khoury. Theory of Dark Matter Superfluidity. Physical Review D, 92(10):103510, November 2015.

[2] S. Perlmutter, G. Aldering, G. Goldhaber, R. A. Knop, P. Nugent, P. G. Castro, S. Deustua, S. Fabbro, A. Goobar, D. E. Groom, I. M. Hook, A. G. Kim, M. Y. Kim, J. C. Lee, N. J. Nunes, R. Pain, C. R. Pennypacker, R. Quimby, C. Lidman, R. S. Ellis, M. Irwin, R. G. McMahon, P. Ruiz-Lapuente, N. Walton, B. Schaefer, B. J. Boyle, A. V. Filippenko, T. Matheson, A. S. Fruchter, N. Panagia, H. J. M. Newberg, W. J. Couch, and The Supernova Cosmology Project. Measurements of $\Omega$ and $\Lambda$ from 42 High-Redshift Supernovae. The Astrophysical Journal, 517(2):565, 1999.

[3] Adam G. Riess, Alexei V. Filippenko, Peter Challis, Alejandro Clocchiatti, Alan Diercks, Peter M. Garnavich, Ron L. Gilliland, Craig J. Hogan, Saurabh Jha, Robert P. Kirshner, B. Leibundgut, M. M. Phillips, David Reiss, Brian P. Schmidt, Robert A. Schommer, R. Chris Smith, J. Spyromilio, Christopher Stubbs, Nicholas B. Suntzeff, and John Tonry. Observational Evidence from Supernovae for an Accelerating Universe and a Cosmological Constant. The Astronomical Journal, 116(3):1009, 1998.

[4] C. L. Bennett, D. Larson, J. L. Weiland, N. Jarosik, G. Hinshaw, N. Odegard, K. M. Smith, R. S. Hill, B. Gold, M. Halpern, E. Komatsu, M. R. Nolta, L. Page, D. N. Spergel, E. Wollack, J. Dunkley, A. Kogut, M. Limon, S. S. Meyer, G. S. Tucker, and E. L. Wright. Nine-year Wilkinson Microwave Anisotropy Probe (WMAP) Observations: Final Maps and Results. The Astrophysical Journal Supplement Series, 208(2):20, 2013.

[5] J. Richard Bond, Robert Crittenden, Richard L. Davis, George Efstathiou, and Paul J. Steinhardt. Measuring Cosmological Parameters with Cosmic Microwave Background Experiments. Physical Review Letters, 72:13-16, January 1994.

[6] M. Milgrom. A Modification of the Newtonian Dynamics as a Possible Alternative to the Hidden Mass Hypothesis. The Astrophysical Journal, 270:365-370, July 1983. 
[7] B. Famaey and S. McGaugh. Challenges for $\Lambda$ CDM and MOND. In Journal of Physics Conference Series, volume 437 of Journal of Physics Conference Series, page 012001, April 2013.

[8] Benoît Famaey and Stacy S. McGaugh. Modified Newtonian Dynamics (MOND): Observational Phenomenology and Relativistic Extensions. Living Reviews in Relativity, 15(1):10, September 2012.

[9] Garry W. Angus, Huan Yuan Shan, Hong Sheng Zhao, and Benoit Famaey. On the Proof of Dark Matter, the Law of Gravity, and the Mass of Neutrinos. The Astrophysical Journal Letters, 654(1):L13, 2007.

[10] J. H. Gundlach, S. Schlamminger, C. D. Spitzer, K.-Y. Choi, B. A. Woodahl, J. J. Coy, and E. Fischbach. Laboratory Test of Newton's Second Law for Small Accelerations. Physical Review Letters, 98:150801, April 2007.

[11] L. Berezhiani, B. Famaey, and J. Khoury. Phenomenological Consequences of Superfluid Dark Matter with Baryon-Phonon Coupling. ArXiv e-prints, page arXiv:1711.05748, November 2017.

[12] Scott Dodelson. Modern Cosmology. Academic Press, Elsevier Science, 2003.

[13] Gianfranco Bertone and Dan Hooper. A History of Dark Matter. arXiv e-prints, page arXiv:1605.04909, May 2016.

[14] Isaac Newton. Philosophiae Naturalis Principia Mathematica. J. Societatis Regiae ac Typis J. Streater, 1687.

[15] John Michell. On the Means of Discovering the Distance, Magnitude, \&C. of the Fixed Stars, in Consequence of the Diminution of the Velocity of Their Light, in Case Such a Diminution Should Be Found to Take Place in Any of Them, and Such Other Data Should Be Procured from Observations, as Would Be Farther Necessary for That Purpose. Philosophical Transactions of the Royal Society of London, 74(35), 1784.

[16] B. Lord Kelvin. Baltimore Lectures on Molecular Dynamics and the Wave Theory of Light. https://archive.org/details/baltimorelecture00kelviala, 1904. [Online; accessed 12-May-2019].

[17] B. Lord Kelvin. Baltimore Lectures on Molecular Dynamics and the Wave Theory of Light. Cambridge Library Collection - Physical Sciences. Cambridge University Press, 2010.

[18] H. Poincare. The Milky Way and the Theory of Gases. Popular Astronomy, 14:475-488, October 1906.

[19] F. Zwicky. Die Rotverschiebung von extragalaktischen Nebeln. Helvetica Physica Acta, 6:110-127, 1933. 
[20] F. Zwicky. Republication of: The Redshift of Extragalactic Nebulae. General Relativity and Gravitation, 41:207-224, January 2009.

[21] F. Zwicky. On the Masses of Nebulae and of Clusters of Nebulae. The Astrophysical Journal, 86:217, October 1937.

[22] S. Smith. The Mass of the Virgo Cluster. The Astrophysical Journal, 83:23, January 1936.

[23] E. P. Hubble. Extragalactic Nebulae. The Astrophysical Journal, 64, December 1926.

[24] E. P. Hubble. Realm of the Nebulae. New Haven: Yale University Press, 1936.

[25] F. G. Pease. The Rotation and Radial Velocity of the Central Part of the Andromeda Nebula. Proceedings of the National Academy of Science, 4:21-24, January 1918.

[26] Mike D. Reynolds. Binocular Stargazing. Stackpole Books, 2005.

[27] H. W. Babcock. The Rotation of the Andromeda Nebula. Lick Observatory Bulletin, 19:41-51, 1939.

[28] H. C. van de Hulst, E. Raimond, and H. van Woerden. Rotation and Density Distribution of the Andromeda Nebula Derived from Observations of the 21-cm Line. Bulletin of the Astronomical Institutes of the Netherlands, 14:1, November 1957.

[29] F. D. Kahn and L. Woltjer. Intergalactic Matter and the Galaxy. The Astrophysical Journal, 130:705, November 1959.

[30] V. C. Rubin and W. K. Ford, Jr. Rotation of the Andromeda Nebula from a Spectroscopic Survey of Emission Regions. The Astrophysical Journal, 159:379, February 1970.

[31] K. C. Freeman. On the Disks of Spiral and S0 Galaxies. The Astrophysical Journal, 160:811, June 1970.

[32] D. H. Rogstad and G. S. Shostak. Gross Properties of Five Scd Galaxies as Determined from 21-Centimeter Observations. The Astrophysical Journal, 176:315, September 1972.

[33] R. N. Whitehurst and M. S. Roberts. High-Velocity Neutral Hydrogen in the Central Region of the Andromeda Galaxy. The Astrophysical Journal, 175:347, July 1972 .

[34] M. S. Roberts and A. H. Rots. Comparison of Rotation Curves of Different Galaxy Types. Astronomy and Astrophysics, 26:483-485, August 1973. 
[35] M. S. Roberts. The Rotation Curves of Galaxies. Symposium - International Astronomical Union, 69:331-340, 1975.

[36] A. Bosma. The Distribution and Kinematics of Neutral Hydrogen in Spiral Galaxies of Various Morphological Types. PhD thesis, Groningen University, 1978.

[37] V. C. Rubin, W. K. Ford, Jr., and N. Thonnard. Extended Rotation Curves of High-Luminosity Spiral Galaxies. IV - Systematic Dynamical Properties, SA Through SC. The Astrophysical Journal Letters, 225:L107-L111, November 1978.

[38] S. M. Faber and J. S. Gallagher. Masses and Mass-to-Light Ratios of Galaxies. Annual review of astronomy and astrophysics, 17:135-187, 1979.

[39] C. J. Pethick and H. Smith. Bose-Einstein Condensation in Dilute Gases. Cambridge University Press, 2. edition, 2008.

[40] W. Pauli. The Connection Between Spin and Statistics. Physical Review, 58:716722, October 1940.

[41] C. A. Regal, M. Greiner, and D. S. Jin. Observation of Resonance Condensation of Fermionic Atom Pairs. Physical Review Letters, 92:040403, January 2004.

[42] Robert P. Smith. Effects of Interactions on Bose-Einstein Condensation. arXiv e-prints, page arXiv:1609.04762, September 2016.

[43] Weisstein, Eric W. from MathWorld - A Wolfram Web Resource. Gradient Theorem. http://mathworld.wolfram.com/GradientTheorem.html, 2019. [Online; accessed 8-March-2019].

[44] M.L. Boas. Mathematical Methods in the Physical Sciences. Wiley, 2005.

[45] Nobel Media AB. Additional Background Material on the Nobel Prize in Physics 1996. https://www.nobelprize.org/prizes/physics/1996/ advanced-information/, 2019. [Online; accessed 16-May-2019].

[46] J.F. Allen and A.D. Misener. Flow of Liquid Helium II. Nature, 141:75 EP -, January 1938.

[47] S. Balibar. The Discovery of Superfluidity. Journal of Low Temperature Physics, 146:441-470, March 2007.

[48] P. Kapitza. Viscosity of Liquid Helium below the $\lambda$-Point. Nature, 141:74 EP -, January 1938.

[49] S. S. McGaugh. The Baryonic Tully-Fisher Relation of Gas-rich Galaxies as a Test of $\Lambda$ CDM and MOND. The Astronomical Journal, 143:40, February 2012.

[50] R. B. Tully and J. R. Fisher. A New Method of Determining Distances to Galaxies. Astronomy and Astrophysics, 54:661-673, February 1977. 
[51] G. W. Angus, A. Diaferio, B. Famaey, and K. J. van der Heyden. Cosmological Simulations in MOND: the Cluster Scale Halo Mass Function with Light Sterile Neutrinos. Monthly Notices of the Royal Astronomical Society, 436(1):202-211, 2013.

[52] G. W. Angus, B. Famaey, and D. A. Buote. X-ray Group and Cluster Mass Profiles in MOND: Unexplained Mass on the Group Scale. Monthly Notices of the Royal Astronomical Society, 387(4):1470-1480, 2008.

[53] Douglas Clowe, Maruca Bradac, Anthony H. Gonzalez, Maxim Markevitch, Scott W. Randall, Christine Jones, and Dennis Zaritsky. A Direct Empirical Proof of the Existence of Dark Matter. The Astrophysical Journal Letters, 648(2):L109, 2006.

[54] R. H. Sanders. The Virial Discrepancy in Clusters of Galaxies in the Context of Modified Newtonian Dynamics. The Astrophysical Journal Letters, 512(1):L23, 1999.

[55] A. Schmitt. Introduction to Superfluidity - Field-Theoretical Approach and Applications. ArXiv e-prints, page arXiv:1404.1284, April 2014.

[56] Emmy Noether. Invariant Variation Problems. Transport Theory and Statistical Physics, 1(3):186-207, 1971.

[57] Michael Edward Peskin and Daniel V. Schroeder. An Introduction to Quantum Field Theory. Westview Press, 1995. Reading, USA: Addison-Wesley (1995) 842 p.

[58] The SciPy Community. scipy.integrate.solve_bvp. https://docs.scipy.org/ doc/scipy-0.18.1/reference/generated/scipy.integrate.solve_bvp.html, 2016. [Online; accessed 11-April-2019].

[59] Julio F. Navarro, Carlos S. Frenk, and Simon D. M. White. The Structure of Cold Dark Matter Halos. The Astrophysical Journal, 462:563, May 1996.

[60] M. López-Corredoira, C. Allende Prieto, F. Garzón, H. Wang, C. Liu, and L. Deng. Disk Stars in the Milky Way Detected Beyond $25 \mathrm{kpc}$ From its Center. Astronomy and Astrophysics, 612:L8, May 2018.

[61] S. Sahu and D. Majumder. Bose-Einstein Condensation in Nonuniform Rotation. ArXiv e-prints, page arXiv:1805.02417, May 2018.

[62] Laura L. Watkins, Roeland P. van der Marel, Sangmo Tony Sohn, and N. Wyn Evans. Evidence for an Intermediate-Mass Milky Way from Gaia DR2 Halo Globular Cluster Motions. The Astrophysical Journal, 873:118, March 2019.

[63] S. Chandrasekhar. The Virial Theorem in Hydromagnetics. Journal of Mathematical Analysis and Applications, 1(2):240 - 252, 1960. 
[64] G. W. Collins, II. The Virial Theorem in Stellar Astrophysics. Pachart Publishing House, 1978.

[65] Weisstein, Eric W. from MathWorld - A Wolfram Web Resource. Lane-Emden Differential Equation. http://mathworld.wolfram.com/ Lane-EmdenDifferentialEquation.html, 2019. [Online; accessed 11-April2019].

[66] Planetary Science Communications team at NASA's Jet Propulsion Laboratory and Goddard Space Flight Center for NASA' Science Mission Directorate. Europa. https://solarsystem.nasa.gov/moons/jupiter-moons/europa/ by-the-numbers/, 2019. [Online; accessed 24-May-2019].

[67] Wayne Hu, Rennan Barkana, and Andrei Gruzinov. Fuzzy Cold Dark Matter: The Wave Properties of Ultralight Particles. Physical Review Letters, 85:11581161, August 2000.

[68] Vincent B. Klaer and Guy D. Moore. The Dark-Matter Axion Mass. Journal of Cosmology and Astro-Particle Physics, 2017:049, November 2017.

[69] CERN. Accelerators. https://home.cern/science/accelerators, 2019. [Online; accessed 5-March-2019].

[70] CERN. Superconductivity. https://home.cern/science/engineering/ superconductivity, 2019. [Online; accessed 14-May-2019].

[71] A. A. Abrikosov. Nobel lecture: Type-II Superconductors and the Vortex Lattice. Reviews of Modern Physics, 76:975-979, December 2004.

[72] Chi Xiong, Michael R. R. Good, Yulong Guo, Xiaopei Liu, and Kerson Huang. Relativistic Superfluidity and Vorticity from the Nonlinear Klein-Gordon Equation. Physical Review D, 90:125019, December 2014.

[73] D. E. Galli, L. Reatto, and M. Rossi. Quantum Monte Carlo Study of a Vortex in Superfluid He4 and Search for a Vortex State in the Solid. Physical Review B, 89(22):224516, June 2014.

[74] Francesco Ancilotto, Martí Pi, and Manuel Barranco. Vortex Arrays in a Rotating Superfluid ${ }^{4}$ He Nanocylinder. Physical Review B, 90(17):174512, November 2014.

[75] S. Villerot, B. Castaing, and L. Chevillard. Static Spectroscopy of a Dense Superfluid. Journal of Low Temperature Physics, 169(1-2):1-14, October 2012.

[76] Robert Hagala. Ph.D. Research Fellow at the Institute of Theoretical Astrophysics at the University of Oslo. Personal communication, 2019.

[77] Nikolaj Thomas Zinner. Vortex Structures in a Rotating BEC Dark Matter Component. arXiv e-prints, page arXiv:1108.4290, August 2011. 
[78] David Harvey, Richard Massey, Thomas Kitching, Andy Taylor, and Eric Tittley. The Nongravitational Interactions of Dark Matter in Colliding Galaxy Clusters. Science, 347:1462-1465, March 2015.

[79] Benoit Famaey. Personal communication, March 2019.

[80] C. G. Böhmer and T. Harko. Can Dark Matter be a Bose Einstein Condensate? Journal of Cosmology and Astro-Particle Physics, 2007(6):025, June 2007.

[81] Silverman, M. P. and Mallett, R. L. Dark Matter as a Cosmic Bose-Einstein Condensate and Possible Superfluid. General Relativity and Gravitation, 34(5):633649, May 2002.

[82] Nikolaj Thomas Zinner. Vortex Structures in a Rotating BEC Dark Matter Component. arXiv e-prints, page arXiv:1108.4290, August 2011.

[83] Partha Nag, Somnath Bharadwaj, and Sayan Kar. Can the Rotation of the Dark Matter Halo of our Galaxy be Detected Through its Effect on the Cosmic Microwave Background Polarisation? arXiv e-prints, pages astro-ph/0506009, June 2005.

[84] Ji-hoon Kim, Oscar Agertz, Romain Teyssier, Michael J. Butler, Daniel Ceverino, Jun-Hwan Choi, Robert Feldmann, Ben W. Keller, Alessandro Lupi, Thomas Quinn, Yves Revaz, Spencer Wallace, Nickolay Y. Gnedin, Samuel N. Leitner, Sijing Shen, Britton D. Smith, Robert Thompson, Matthew J. Turk, Tom Abel, Kenza S. Arraki, Samantha M. Benincasa, Sukanya Chakrabarti, Colin DeGraf, Avishai Dekel, Nathan J. Goldbaum, Philip F. Hopkins, Cameron B. Hummels, Anatoly Klypin, Hui Li, Piero Madau, Nir Mandelker, Lucio Mayer, Kentaro Nagamine, Sarah Nickerson, Brian W. O'Shea, Joel R. Primack, Santi RocaFàbrega, Vadim Semenov, Ikkoh Shimizu, Christine M. Simpson, Keita Todoroki, James W. Wadsley, John H. Wise, and AGORA Collaboration. The AGORA High-resolution Galaxy Simulations Comparison Project. II. Isolated Disk Test. The Astrophysical Journal, 833(2):202, December 2016.

[85] Sabine Hossenfelder and Tobias Mistele. Strong Lensing with Superfluid Dark Matter. Journal of Cosmology and Astro-Particle Physics, 2019(2):001, February 2019.

[86] Rong-Gen Cai, Tong-Bo Liu, and Shao-Jiang Wang. Gravitational Wave as Probe of Superfluid Dark Matter. Physical Review D, 97(2):023027, February 2018.

[87] Elisa G. M. Ferreira, Guilherme Franzmann, Justin Khoury, and Robert Brandenberger. Unified Superfluid Dark Sector. arXiv e-prints, page arXiv:1810.09474, October 2018.

[88] Stian Hartman. Ph.D. Research Fellow at the Institute of Theoretical Astrophysics at the University of Oslo. Personal communication, 2019. 
[89] A. W. Manyonge, R. Opiyo, D. Kweyu, and J. S. Maremwa. Numerical Solution of Non-Linear Boundary Value Problems of Ordinary Differential Equations Using the Shooting Technique. Journal of Innovative Technology and Education, 4(1):29$36,2017$.

[90] R.L. Burden and J.D. Faires. Numerical Analysis, chapter 11. Brooks/Cole, Cengage Learning, 9th edition, 2010.

[91] Wolfram Language \& System - Documentation Center . Numerical Solution of Boundary Value Problems (BVP). https://reference.wolfram.com/language/ tutorial/NDSolveBVP.html, 2019. [Online; accessed 20-May-2019].

[92] U.M. Ascher and L.R. Petzold. Computer Methods for Ordinary Differential Equations and Differential-Algebraic Equations, chapter 4, pages 101-102. Other Titles in Applied Mathematics. Society for Industrial and Applied Mathematics (SIAM, 3600 Market Street, Floor 6, Philadelphia, PA 19104), 1998.

[93] A. Nedich and U. Shanbhag. Lecture Notes in Game Theory: Models, Algorithms and Applications. http://www.ifp.illinois.edu/ angelia/ie598ns_lect14_ 2.pdf, 2008. [Online; accessed 20-May-2019].

[94] Nikolay Mayorov. Boundary Value Problem Solver: scipy.integrate.solve_bvp. https://github.com/scipy/scipy/blob/v0.18.1/scipy/integrate/_bvp. py\#L714-L1134, 2016.

[95] John Christian Haukenes Skorgan. Master Student at the Institute of Theoretical Astrophysics at the University of Oslo. Personal communication, 2019. 